\title{
Biophysics of Blood Platelet Contraction
}

\author{
Dissertation \\ for the award of the degree \\ "Doctor rerum naturalium" (Dr. rer. nat.) \\ Division of Mathematics and Natural Sciences \\ of the Georg-August-Universität Göttingen \\ submitted by \\ Sarah Michaela \\ Schwarz Gonçalves Henriques \\ from Madison (WI, USA)
}

Göttingen; 31st May 2012

Institute for X-Ray Physics

Georg-August-Universität Göttingen 


\section{Thesis Committee:}

Sarah Köster (supervisor and 1st reviewer)

Georg-August-Universität Göttingen

Physics Department

Institute for X-Ray Physics

Stephan Herminghaus (2nd reviewer)

Max-Planck Institute for Dynamics and Self Organization

Department Dynamics of Complex Fluids

Tim Salditt

Georg-August-Universität Göttingen

Physics Department

Institute for X-Ray Physics

\section{Date of oral examination:}

10.07 .2012 
I hereby declare that the present thesis is my own work in its entirety; no part has been written by or copied from others. Furthermore, I certify that any material included from the work of other people has been duly referenced and acknowledged. Apart from the references contained in the thesis, no other sources or aids have been used.

Göttingen, 31st May 2012 

This work is part of a project on blood platelet contraction using an innovative double approach to experiments. First, we adapted the experimental technique, traction force microscopy (TFM), to our cellular system, and measured forces generated by single, activated platelets with hitherto unknown spatial and temporal resolution. In a second set of experiments, we looked at both force generators and force transduction sites (focal adhesions) in platelets that were activated on soft polyacrylamide (PAA) substrates, used in kind already for TFM. Here, we fixed platelets and stained platelet components during different stages of activation. By combining these two experimental approaches, we were able to establish connections between characteristics of measured force fields and the (re-)organization of both the platelet cytoskeleton and focal adhesion sites.

Our findings have several implications for experimental concepts applied to future force measurements of platelet contraction. In addition, our experiments on PAA have revealed that platelet activation on materials with physiological elasticities proceeds in a slightly different manner than on glass, the traditional choice of substrate. Lastly, our measurements of local forces illustrate specific characteristics of platelet force fields, which will affect future mechanical models of platelet contraction.

Chapter I introduces platelets as a cellular system, furthermore summarizing what is known to date about mechanisms of cellular contraction in general, and platelet contraction in particular. In addition, we outline the objectives of this project and explain about the experimental techniques used. For one of the experimental techniques (TFM) we had to make a few adjustments, an overview of which is also provided in this chapter. The relevant theoretical background for TFM is presented in chapter II, in which we also refer to theoretical, state-of-the-art models for cellular contraction. Sample preparation and measuring procedures are explained in chapter III, and steps taken during data analysis are detailed in chapter IV. Here in particular, we refer to programs we have developed to be able to apply TFM to platelet contraction. The experimental results are then presented in chapter $\mathrm{V}$, which is followed by a discussion and interpretation of our findings in chapter VI. Finally, we summarize our conclusions in chapter VII and conclude with suggestions for improvements and further experiments. 



\section{List of Abbreviations}

$\alpha-\mathrm{G} \ldots \ldots . \quad \alpha$-granules - a type of platelet granule; $\mathrm{p} .3$

AA $\ldots . . . .$. arachidonic acid; p. 11

ACN ....... active cable networks - mechanical network model; p. 38

ADP ....... adenosine diphosphate; p. 4

AF ....... actin filaments; $p .3$

AFM $\ldots . .$. atomic force microscopy; p. 22

AMP ....... adenosine monophosphate; p. 17

Arp2 $/ 3 \ldots$ protein with actin-related sequences in the subunits Arp2 and Arp3;

p. 7

ATP ....... adenosine triphosphate; p. 4

cAMP ...... cyclic adenosine monophosphate; p. 13

cfu .......... colony-forming units; p. 48

COX ...... cyclooxygenase - PGHS in one of its catalytic functions; p. 11

CPD ........ citrate-phosphate-dextrose; p. 47

DAG $\ldots . .$. diacylglycerol; p. 7

DB ....... dense bodies - a type of platelet granule; $p .3$

DIC ....... differential interference contrast; p. 10

DTS ....... dense tubular system in platelets; p. 4

F-actin ..... filamentous actin; p. 52

FEM ...... finite-element-method; p. 28

G-protein ... GTP-binding protein; p. 13

GDP ...... guanosine diphosphate; p. 13

GFP ....... green fluorescent protein; p. 28

GL $\ldots \ldots \ldots$ glycogen; p. 3

GLS $\ldots . .$. glycosome; p. 3

GP $\ldots . . \ldots$ glycoprotein; p. 2

GPCR ..... receptors coupling to GTP-binding proteins (G proteins); p. 12

GTP ....... guanosine triphosphate; p. 12

HBSS ....... Hank's balanced salt solution; p. 54

HEPES .... 4-(2-hydroxyethyl) piperazine-1-ethanesulfonic acid; p. 47

HOX ...... hydroperoxidase - PGHS in one of its catalytic functions; $p$. 11

HSN ....... Hookean spring networks - mechanical network model; p. 38

$\mathrm{IP}_{3} \ldots \ldots \ldots$ inositol triphosphate; $\mathrm{p} .7$

$\mathrm{L} \ldots \ldots \ldots$ lysosome - a type of platelet granule; $\mathrm{p} .3$

$\mathrm{M} \ldots \ldots \ldots$ mitochondrion; $\mathrm{p} .3$

MAP ...... mitogen-activated protein; p. 11

MLC ...... myosin light chain; p. 14

MLCK ..... myosin light chain kinase; p. 15 
MT ....... microtubules; p. 3

NHS ester .. acrylic acid N-hydroxysuccinimide ester; p. 48

NO ....... nictric oxide; $p .5$

NTP ....... nucleoside triphosphate; p. 17

OCS $\ldots \ldots \ldots$ open canalicular system in platelets; $p .2$

PAA $\ldots . . .$. polyacrylamide; p. 26

PAR ....... protease-activated receptor; p. 12

PBS ....... phosphate buffered saline - recipe p. 48

PCN ...... passive cable networks - mechanical network model; p. 38

PG ....... prostaglandin; p. 5

PGHS ..... prostaglandin endoperoxide $\mathrm{H}$ synthase, depending on its catalytic function: also called COX or HOX; p. 11

PI ........ phosphatidylinositol - a type of phospholipid in platelet membranes; p. 7

PI3K ...... phosphoinositide-3 kinase; p. 7

$\mathrm{PIP}_{2} \ldots \ldots$ phosphatidylinositol 4,5 biphosphate; $\mathrm{p} .7$

PIPES ..... piperazine-N,N'-bis(2-ethanesulfonic acid); p. 47

PKC ....... protein kinase C; p. 7

PL ......... phospholipase; p. 7

ppb ........ parts per billion; p. 48

PSG ....... pipes-saline-glucose - recipe p. 47

RBC ....... red blood cell; p. 6

ROCK ...... rho kinase; p. 15

SHIP-2 .... Src homology 2 domain-containing inositol polyphosphate 5-phosphatase-2; p. 7

SMF ....... submembrane filaments - short actin filaments and associated proteins; p. 3

TEMED .... N,N,N',N'- tetramethyl ethylenediamine - a catalyzer for acrylamide polymerization; p. 48

TFM ...... traction force microscopy; p. 1

TOC ....... total organic carbon; p. 48

$\mathrm{TXA}_{2} \ldots \ldots$ thromboxane $\mathrm{A}_{2} ; \mathrm{p} .11$

VASP ...... vasodilator-stimulated phosphoprotein; p. 9

VWF ...... von Willebrand factor; p. 4

WASp ..... Wiskott-Aldrich syndrome proteins; p. 7 


\section{Contents}

\begin{tabular}{lll}
\hline State-of-the-Art & 1
\end{tabular}

I.1 Biological Background . . . . . . . . . . . . . . . . . . . . 1

I.1.1 $\quad$ Platelet Structure . . . . . . . . . . . . . . . . . . . . 2

I.1.2 Clot Formation . . . . . . . . . . . . . . . . . . . . . . . . . . . 5

$I .2$ Contractile Forces in Cells . . . . . . . . . . . . . . . . . 17

I. 3 Platelet Contraction . . . . . . . . . . . . . . . . . . . . . . 24

$\left[\begin{array}{ll}\hline .4 & \text { Traction Force Microscopy (TFM) } \ldots \ldots \ldots \ldots \ldots \ldots\end{array}\right.$

\begin{tabular}{lll}
\hline 11 & Theory & 31
\end{tabular}

II.1 $\quad$ Elastic Deformation of an Isotropic Medium . . . . . . . . . . . . 31

II.1.1 Thermodynamics of Elastic Deformation. . . . . . . . . . 31

II.1.2 Deformation of a Semi-Infinite Elastic Medium . . . . . . . 34

III.2 $\quad$ Mechanical Principles of Cellular Contraction . . . . . . . . . . . 38

II.2.1 Cell Shape and Force Distribution According to Mechanical

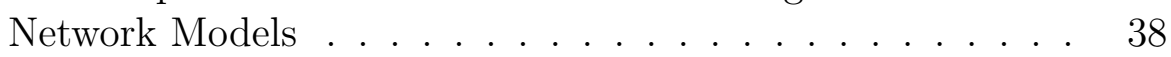

II.2.2 $\quad$ Modeling Stress Fiber Polarization . . . . . . . . . . . . 41

\begin{tabular}{lll}
\hline $1 I$ & Materials and Methods & 47
\end{tabular}

III.1 $\quad$ Subjects and Platelet Isolation . . . . . . . . . . . . . . . . 47

III.2 $\quad$ Preparation of Substrates $\ldots \ldots \ldots \ldots \ldots$

III.3 $\quad$ Substrate Elasticity Measurements _. . . . . . . . . . . . . 50

III.4 Traction Force Microscopy (TFM) _ . . . . . . . . . . . . . 52

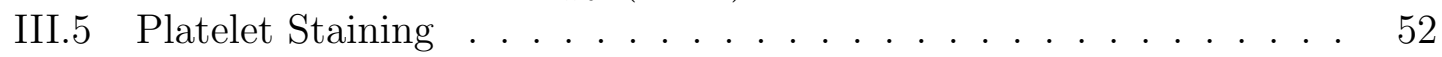

III.5.1 Staining on PAA . . . . . . . . . . . . . . . 52

III.5.2 Control Staining on Glass . . . . . . . . . . . . . . 54

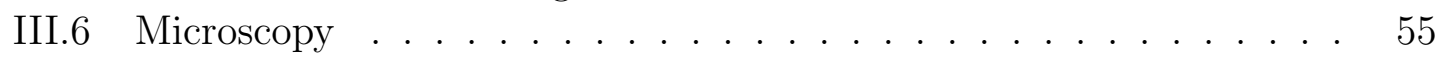

\begin{tabular}{lll}
\hline IV Data analysis & 61
\end{tabular}

IV.1 Image Preprocessing . . . . . . . . . . . . . . . . . . . . 61

IV.2 Bead Traction . . . . . . . . . . . . . . . . . . . . . . . 63

IV.3 Determination of Cellular Contours . . . . . . . . . . . . . . . 67

IV.4 Calculation of Traction Force Fields. . . . . . . . . . . . . 70

IV.5 Determination of the Time Point of Adhesion . . . . . . . . . 74

IV.6 $\quad$ Estimation of the Traction Force Fields' Spatial Resolution. . . . . 75

IV.7 $\quad$ Preparation of Traction Force Field Characterization . . . . . . . 76

IV.7.1 Calculating the Divergence of Traction Force Fields _. . . 76

IV.7.2 Extraction of a Typical Cell Radius $\ldots \ldots \ldots \ldots . . \ldots 77$

IV.8 Processing of Images from Stained Platelets $\ldots \ldots \ldots \ldots \ldots$ 
\begin{tabular}{llr}
\hline V & Results & $\mathbf{8 1}$
\end{tabular}

V.1 Temporal Evolution of Platelet Contraction . . . . . . . . . . . . . 82

V.1.1 Traction Force Evolution . . . . . . . . . . . . . . . . . . . 82

V.1.2 Cytoskeletal Reorganization. . . . . . . . . . . . . . . 85

V.2 $\quad$ Spatial Organization during Platelet Contraction . . . . . . . . . . 87

V.2.1 Distribution of Traction Forces . . . . . . . . . . . . . . . 87

V.2.2 Distribution of Myosin Motors and Focal Adhesions . . . . 89

\begin{tabular}{lll}
\hline VI Discussion and Conclusions & 93
\end{tabular}

VI.1 Limitations of TFM . . . . . . . . . . . . . . . . . . . . . . . . . . . . . . 93

VI.2 $\quad$ Platelet Contractile Forces . . . . . . . . . . . . . . . . . . . . . . . 96

VI.3 $\quad$ Platelet Force Generators . . . . . . . . . . . . . . . . . . . . . . . 97

VI.4 Mechanical Models of Platelet Contraction . . . . . . . . . . . . . . 100

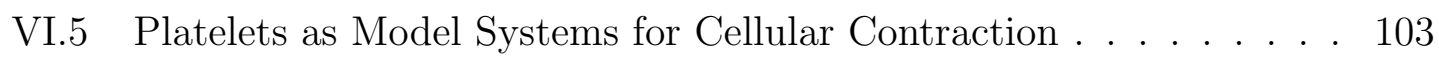

\begin{tabular}{lll}
\hline VII Summary and Outlook & 105
\end{tabular}

\begin{tabular}{|l|l}
\hline VIII Acknowledgements & 111
\end{tabular}

\begin{tabular}{ll}
\hline Bibliography & 113
\end{tabular} 


\section{State-of-the-Art}

Cellular contraction is vital to living organisms. It regulates not only our mobility through muscle cell contraction and our blood circulation through the contraction of cardiac tissue in our heart, but it also plays a role in wound healing. In particular, blood platelets, the main agents of hemostasis, contract in the final stages of clot formation to solidify the clot mass. Apart from their medical importance, platelets represent a very suitable model system to study cellular contraction for three main reasons. First, they are simple cells lacking a nucleus. Secondly, platelet activation is fast, occurring within minutes and finally platelet activation can be initiated and synchronized by the addition of thrombin. In the following chapter, details will be given on the biological background of platelets, their origin, their structure and their in vivo function, based on the information available from reference [38] (mainly chapters 1-4, 7-9, 13, 15-19 and 31). We will then summarize state-of-the-art measurements of contractile forces that were performed on other cell types and cellular components, before we turn to what is known to date of platelet contraction. Finally, we will present the experimental method we use to measure contractile forces, traction force microscopy (TFM), and explain the different approaches to evaluate TFM data.

\section{I.1 Biological Background}

Blood platelets, when activated, are the cells that perform blood clotting to stop bleeding (hemostasis) at the site of blood vessel injury. According to reference [38, chapter 1], the name "platelets" is used to distinguish the annucleated mammalian cells from the equivalent nucleated cells in non-mammalian vertebrates. Platelets are $2-5 \mu \mathrm{m}$ in diameter, $0.5 \mu \mathrm{m}$ in thickness and have a volume of 6-10 $\mathrm{fl}$ when they are in their quiescent, discoid shape [38, p. 45]. They have a lifespan of 7-10 days [38, p. 45] and on average, a person has $(150-400) \cdot 10^{9}$ platelets per liter [38, p. 475]. Their origin lies in the megakaryocytes from bone marrow [38, chapter 2], which are large cells that develop multiple nuclei (polyploid) and massively reorganize their cytoplasm into beaded protrusions, so called proplatelets. Proplatelets are most likely the precursors of platelets. Although platelet formation and release are not fully understood yet, it is believed that megakaryocytes extend proplatelets into blood circulation, where the ends of the proplatelets are severed. The final platelet maturation from these megakaryocyte fragments may, however, occur within blood circulation. This particular mechanism of platelet formation from megakaryocytes is believed to be important for the rapid release of larger than normal platelets, which are biologically more active and which are released in cases of pathophysiological emergency [38, p. 18].

Apart from their primary hemostatic function, platelets also play a minor role in the immune response of the body [38, chapter 1 and 40]. Thus, they contribute to 
inflammation [38, chapter 1 and 39] and they were found to interact with endotoxins, bacteria, viruses, parasites and fungi, which can lead to platelet aggregation, release of platelet constituents, the internalization of the alien substance (phagocytosis) and ultimately a shortening of the platelet life span.

\section{I.1.1 Platelet Structure}

In their inactive, resting state platelets have a discoid shape, the outer surface of which looks smooth under the electron microscope except for deep tunnel-like membrane invaginations randomly distributed across the platelet surface that mark the entrances to an extensive, interconnected channel system in the platelets' interior, the open canalicular system (OCS) (figure I.1 b)). The OCS is believed to be involved in uptake by platelets, transfer of products and the secretion of granular content to the extracellular environment. Additionally, it is a membrane reservoir that is likely used for platelet spreading over the injured blood vessel surface during platelet hemostatic response. The plasma membrane which makes up the outer surface of the platelet consists of three components: the glycocalix, a lipid bilayer and the submembrane filaments in conjuncture with spectrin (figure I.1 c), see also reference [38, chapter 3]). The glycocalix is covered with glycoprotein (GP) receptors, such as the adhesion-mediating GPIb-IX-V (25,000/platelet) and the aggregating agent $\alpha \mathrm{IIb} \beta 3$ (also denoted GPIIbIIIa; 80,000/platelet) [38, p. 47, 189]. Both receptors are connected to the submembrane filaments; GPIb-IX-V via filamin $\left[{ }^{[1]}\right.$ and $\alpha \operatorname{IIb} \beta 3$ via the cytoplasmic tails of $\alpha \mathrm{IIb}$ and $\beta 3$. These links to the submembrane region reach through the lipid bilayer and the holes in a 2-D spectrin network that lines the cytoplasmic side of the bilayer. The spectrin network is composed of $\sim 2000$ spectrin molecules that form strands, which are interconnected at the ends of submembrane filaments, most likely with the help of adducin [38, p. 77]. The submembrane filaments themselves are short actin filaments associated with myosin and calmodulin, which represent the submembrane contractile system of platelets. Directly below the submembrane filaments and running the circumference of the resting, discoid platelet is a bundled microtubule coil consisting of a single microtubule of a diameter of $25 \mathrm{~nm}$, which is looped about itself in 8-12 loops [38, p. 53 and 81] (figure I.1 a)). Most of the tubulin found in resting platelets is present in that microtubule coil, which provides the stability of the discoid shape of human platelets.

Confined within the plasma membrane and the microtubule coil, the interior of the platelet presents itself with mechanical properties similar to a liquid gel. It is spanned by a dense fibrous, cytoplasmic actin network, which together with the actin in the submembrane filaments makes up half of the total actin content in platelets $\left(\sim 2 \cdot 10^{6}\right.$ actin molecules/platelet [38, p. 79]). The remaining half is present in resting platelets in its non-filamentous form. The cytoplasmic actin network is probably highly crosslinked mostly by $\alpha$-actinin. It represents a scaffold for organelles, keeping them apart and randomly distributed across the cell's interior (figure I.1 a)). These organelles can be divided into three categories: $\alpha$ granules (usually $40-80 /$ platelet, $200-500 \mathrm{~nm}$ in di-

\footnotetext{
${ }^{1}$ GPIb-IX-V-filamin links make up $85 \%$ of the linkage between the actin cytoskeleton and the plasma membrane [38, p. 88].
} 
a)

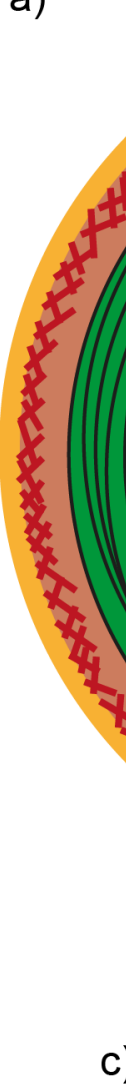

c) b)

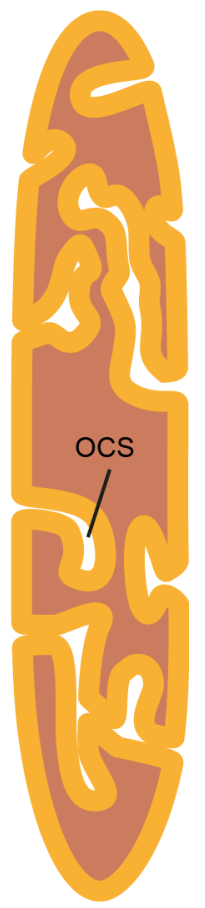

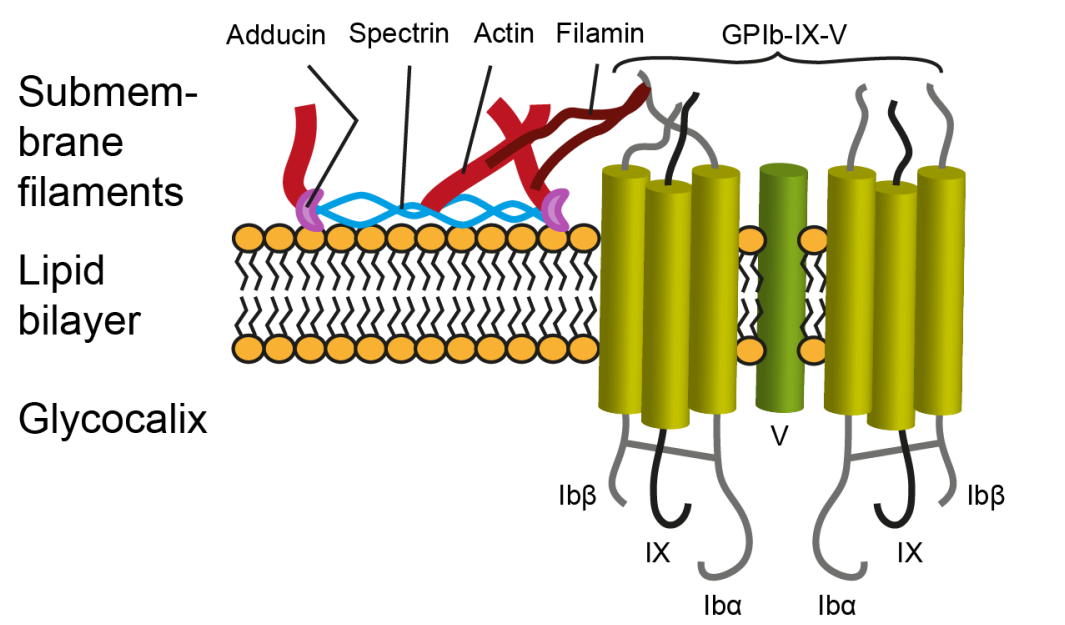

Figure I.1: Internal structure of the resting platelet: a) Cross section of the platelet's interior containing: submembrane filaments (SMF), a tightly wound microtubule coil (MT), cytosolic actin filaments (AF), the membranous channel systems of the open canalicular system (OCS) and the dense tubular system (DTS), mitochondria (M), glycogen particles (GL), glycosomes (GLS) and organelles such as $\alpha$ granules $(\alpha-\mathrm{G})$, dense bodies (DB) and lysosomes (L); b) Another cross sectional view of the platelet showing the channels of the OCS; c) Zoom-in showing the three layers constituting the outer platelet membrane: the glycocalix with membrane receptors such as GPIb-IX-V, the lipid bilayer and the SMF. The SMF are composed of short actin filaments and their associated proteins. In between the SMF and the lipid bilayer lies a spectrin network spanning the inner surface of the platelets. [Figure design based both on sketches and electron microscopy images in [38] (chapter 3 and p. 76 and 322)] 
ameter - [38, pp. 59]), dense bodies or $\delta$ granules (4-8/platelet in humans, $\sim 150 \mathrm{~nm}$ - [38, p. 62 and 77]) and lysosomes (usually 0-1/platelet in humans - [38, p. 64]). In humans, the spherical to oval $\alpha$ granules consist of a denser central zone with proteins that might have binding sites for heavy metals and a less dense peripheral zone containing proteins such as coagulation factor $\mathrm{V}$ and the adhesive proteins von Willebrand factor (VWF), fibronectin and fibrinogen [38, p. 310]. In their membrane, $\alpha$ granules furthermore store P-selectin, the receptor complex GPIb-IX-V and $\alpha \operatorname{IIb} \beta 3$. A little smaller than $\alpha$ granules, dense bodies in human platelets can assume a number of different shapes (figure I.1 a)). At their core they have a very dense region, which is surrounded by a layer that appears transparent under an electron microscope. The two regions are in turn engulfed by a membrane, which also contains P-selectin in small amounts. Dense bodies carry adenine nucleotides such as ATP and ADP, as well as serotonin, pyrophosphate, magnesium and particularly abundantly in humans, calcium [38, p. 310]. Finally, the less well known lysosomes encase a number of components, all of which apparently are not necessary for platelet hemostatic response. In fact, the role of lysosomes is poorly understood. They may function as digestion compartments after phagocytosis, but it is more likely that they are an evolutionary remnant of no great importance. In addition to granular organelles, the platelet interior contains a few vesicles and a rich amount of scattered single glycogen particles and masses of glycogen particles. These masses of glycogen are often associated with membrane segments (figure I.1 a)). Completely membrane-enclosed accumulations of glycogen particles are also found in platelets in the form of glycosomes. Glycogen as a precursor of glucose represents the energy stores in the cell. Energy generation is then performed by the mitochondria, which are present in the platelet's interior in small numbers and simple structures, nevertheless fully meeting the platelet's energy requirements (figureI.1 a)). Mitochondria in platelets were furthermore found to contain calcium and were postulated to be the calcium source in platelets. However, experimental evidence rather suggests that the dense tubular system in the platelet is responsible for providing calcium. The name dense tubular system (DTS) refers to a number of channels that are randomly distributed across the platelet cytoplasm and which are distinguishable from the OCS by the amorphous material that they contain within themselves (figure I.1 a)). Apart from calcium binding sites, they are also believed to incorporate enzymes related to prostaglandin synthesis. Although there are no direct connections between the DTS and the OCS, the two systems appear often geographically interspersed in platelets. While the OCS is in its composition part of the plasma membrane, the DTS is a remnant from channels of the rough and smooth endoplasmic reticulum of megakaryocytes. In general, platelets do not retain any rough endoplasmic reticulum of their own, nor any ribosomal complexes, so that they seem to be unable to synthesize new proteins. Compared to other cells, also absent in platelets are Golgi complexes for packaging of proteins.

Apart from the main components, which have been presented so far, some physiologically less important elements are found in platelets, such as electron-dense chains and clusters as well as tubular inclusions. More details on these and on platelet structure in general can be found in [38, chapter 3]. 


\section{I.1.2 Clot Formation}

In blood flow, platelets are pushed by fluid shear forces to the vessel wall [38, p. 75]. This puts them in an ideal position to recognize sites of injury in the endothelial lining of the vessel wall (figure I.2 a)). When they encounter an injury they activate at the damaged site and adhere to the subendothelial matrix. Upon adhesion they loose their discoid shape by polymerizing actin; they round up to a spherical shape and extend fingerlike protrusions (filopodia) before spreading over the wounded surface. In addition, an activated platelet releases chemical attractants, recruits and activates adhesive membrane receptors and furthermore uses its filopodia to capture and bind additional platelets to the wounded site (figure I.2 b)). The early inter-platelet connections that are thus formed, are mostly established via fibrinogen, which is later transformed into fibrin strands. As the platelet-fibrin(ogen) aggregate grows, other cells such as red blood cells are incorporated into the mesh giving clots their red coloring (figure I.2 b)). Finally, platelets contract with the help of actin-associated myosin activity to solidify the cell aggregate mass and transform it into an efficient plug. This contraction serves the additional purpose of bringing the wound edges closer together and pulling the constricting clot mass against the vessel wall and out of the blood stream [8]. Overall, clot formation is based on a series of complex signaling cascades (see also [38, chapter 16 and 17]). Although some details on these cascades still remain elusive, a brief summary will subsequently be given of what is known to date of the regulatory signaling underlying the different and overlapping stages of clot formation.

\section{Clot Initiation}

Platelet activation can be initiated by a number of external cues. In fact, the endothelial cells at the blood vessel wall are constantly secreting nitric oxide (NO) and prostaglandin $\mathrm{I}_{2}\left(\mathrm{PGI}_{2}\right.$, also called prostacyclin) into the blood flow to prevent premature platelet adhesion, activation and aggregation (figure I.2 a), see also [38, chapter 13]). This inhibitory activity of endothelial cells is complemented by the resting platelets themselves, which also extrude NO is small amounts. Interestingly, the membrane receptor IP on the platelet, that binds $\mathrm{PGI}_{2}$, is not specific to it, also binding other prostaglandins such as $\mathrm{PGE}_{1}$ and $\mathrm{PGE}_{2}$, albeit with different affinities. The platelet inhibiting effects of $\mathrm{PGI}_{2}$ can therefore also be achieved by addition of $\mathrm{PGE}_{1}$, an antagonist that is often used in in vitro experiments (see section III.1). At a damaged blood vessel site, however, the endothelial cells are locally missing and so are the antagonists of platelets that they release. Furthermore, the subendothelium containing the platelet agonist collagen is exposed. Platelets bind to collagen either directly by means of the integrin receptor $\alpha 2 \beta 1$ (also called GPIa-IIa) and GPVI or indirectly via platelet receptors GPIb-IX-V and $\alpha \operatorname{Ib} \beta 3$ that bind to linearized von Willebrand factor (VWF), which in turn is bound to collagen[2] (figure I.3). Of the four receptors, GPVI and GPIb-IX-V are the first to bind to collagen, triggering platelet activation

\footnotetext{
${ }^{2}$ Under static conditions platelets adhere to collagen directly, but under blood flow the direct bond is no longer securely established and the intermediate step of binding to VWF to capture platelets becomes important. VWF is bound by the most abundant receptor proteins on platelets, $\alpha \operatorname{IIb} \beta 3$ and GPIb-IX-V, and it is multimeric, that is, it provides several binding sites per VWF molecule. Hence, VWF increases the likelihood of platelet binding to collagen [38, chapter 16]. VWF obtains its
} 
a)

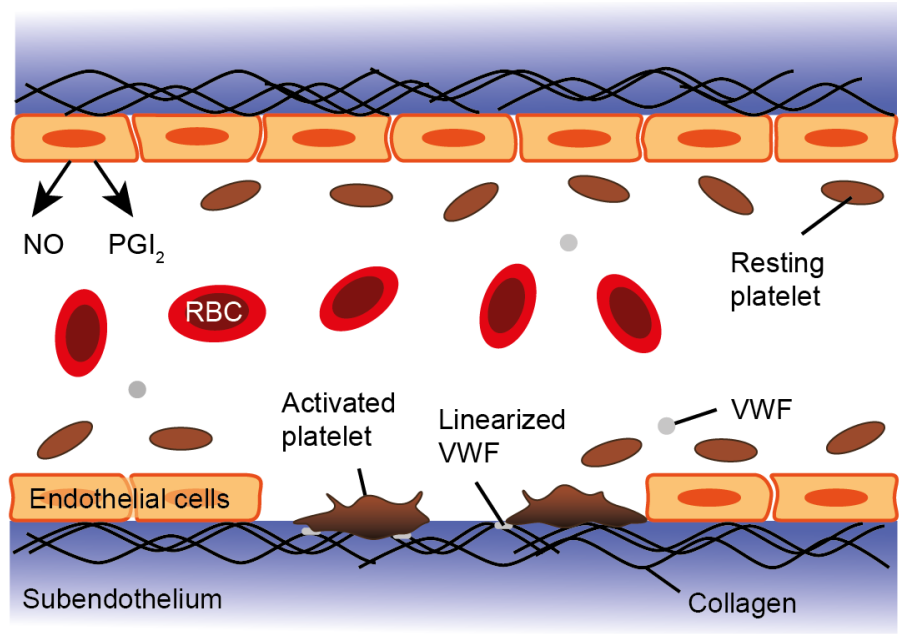

b)

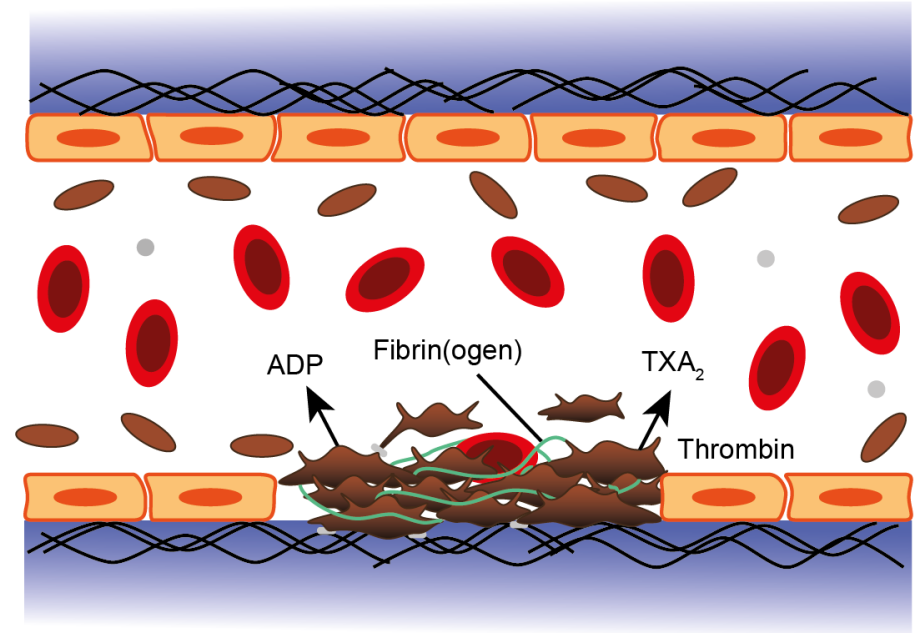

Figure I.2: Overview of in vivo blood clotting: If the endothelial lining of blood vessels is damaged, the platelet inhibiting factors nitric oxide $(\mathrm{NO})$ and prostaglandin $\mathrm{I}_{2}\left(\mathrm{PGI}_{2}\right)$ that are secreted by the endothelium are locally absent (a). Furthermore, the subendothelial platelet agonist collagen is exposed so that blood circulating platelets can recognize and adhere to the site of injury. Adhesion to collagen can then occur directly or via linearized von Willebrand factor (VWF). Once adhered to collagen, platelets activate, changing their shape by extending fingerlike filopodia and by spreading on the wound surface. Simultaneously platelets secrete further attractants, such as the nucleotide ADP and thromboxane $\mathrm{A}_{2}\left(\mathrm{TXA}_{2}\right)$ and accelerate the generation of the clotting factor thrombin (b). By these means and by activating adhesive receptors on their membrane, activated platelets can recruit additional cells into the site of injury. Early platelet-platelet connections are subsequently established through fibrinogen, which is later converted into insoluble fibrin fibers. As the platelet-fibrin clot mass grows, other cells such as red blood cells (RBCs) are incorporated into the mesh. In a final step, platelets contract pulling on their fibrin links to solidify the clot. [Figure adapted from sketch in [38, p. 320]] 
and internal signaling $[3]$, which ultimately activates $\alpha 2 \beta 1$ and $\alpha \operatorname{Ib} \beta 3$. Once activated $\alpha 2 \beta 1$ and $\alpha \operatorname{IIb} \beta 3$ can reinforce adhesion to collagen and further contribute to internal signaling. Although, the internal signaling cascade is a little different for each of the four receptors [38, chapter 16 and 17], they all eventually induce the activation of phospholipase $\mathrm{C} \gamma_{2}\left(\mathrm{PLC} \gamma_{2}\right)$ [38, p. 157, 322, 353 and 361] (figure I.3). PLC $\gamma_{2}$ then hydrolyzes the membrane-bound phosphatidylinositol 4,5 biphosphate $\left(\mathrm{PI}_{4,5} \mathrm{P}_{2}\right.$ or $\mathrm{PIP}_{2}$ ), thus generating diacylglycerol (DAG) and inositol triphosphate $\left(\mathrm{IP}_{3}\right)$. DAG, in turn, activates several forms of protein kinase $\mathrm{C}$ (PKC). The other second messenger, $\mathrm{IP}_{3}$, diffuses to the dense tubular system (DTS) in platelets, binds to calcium channels there and thus releases $\mathrm{Ca}^{2+}$ stores into the cytosol. The resulting temporary rise in cellular $\mathrm{Ca}^{2+}$ has again several effects on the cell including activation of $\alpha \mathrm{IIb} \beta 3$, actin reorganization, platelet secretion and activation of myosin motor contraction (figure I.3.

\section{Cytoskeletal reorganization and platelet shape change}

One of the effects of increased $\mathrm{Ca}^{2+}$ concentrations in the cell is that it initiates platelet shape change by triggering cytoskeletal actin assembly [38, chapter 4]. To that end, $\mathrm{Ca}^{2+}$ activates gelsolin (20,000 copies/platelet, [38, p. 83]; see also figure I.3). Gelsolin opens up its inactive, globular form to expose actin binding sites, which it uses to sever filamentous actin, remaining on the barbed end of actin after completing the severing process. By severing existing actin filaments, gelsolin generates new barbed ends increasing the total number of barbed ends by 5 - to 10 -fold as compared to the resting state of platelets [38, p. 86]. After a certain time about $50 \%$ of the gelsolin then dissociates from actin [38, p. 84], exposing the newly generated barbed end and effectively uncovering nucleation sites for actin polymerization. Simultaneously, other barbed-end capping proteins, such as adducin, dissociate from actin, as well. This dissociation correlates with increasing levels of phospholipids $\mathrm{PI}_{4} \mathrm{P}, \mathrm{PI}_{4,5} \mathrm{P}_{2}, \mathrm{PI}_{3,4,5} \mathrm{P}_{3}$ and $\mathrm{PI}_{3,4} \mathrm{P}_{2}$ that bind capping proteins at the plasma membrane of platelets. The levels of $\mathrm{PI}_{4} \mathrm{P}$ and $\mathrm{PI}_{4,5} \mathrm{P}_{2}$ are the first to increase as soon as PLC activity decreases again in the activated platelets following the first 1 to 10 seconds of platelet activation. $\mathrm{PI}_{4} \mathrm{P}$ and $\mathrm{PI}_{4,5} \mathrm{P}_{2}$ then form the basis for the production of both $\mathrm{PI}_{3,4,5} \mathrm{P}_{3}$ and $\mathrm{PI}_{3,4} \mathrm{P}_{2}$. Thus, for example, $\mathrm{PI}_{3,4,5} \mathrm{P}_{3}$ is generated via phosphorylation of $\mathrm{PI}_{4,5} \mathrm{P}_{2}$ by PI-3 kinase (PI3K) [38, p. 87 and 328]. In turn, $\mathrm{PI}_{3,4} \mathrm{P}_{2}$ is formed as a result of hydrolysis of $\mathrm{PI}_{3,4,5} \mathrm{P}_{3}$ by an active complex of the phosphatase SHIP-2, filamin, actin and GPIb-IX-V [38, p. 87, 157]. In addition to uncapping barbed actin ends in the submembrane regions, platelets activate the actin-related protein Arp2/3 probably via Wiskott-Aldrich syndrome proteins (WASp) and via the focal adhesion proteins vinculin and zyxin (figure I.3). One side of Arp2/3 mimics the pointed end of actin filament, so that actin polymerization can occur at the other side of Arp2/3. As Arp2/3 can associate both laterally with exist-

reactive, linearized shape as a result of the shear stresses that the blood flow exerts on it after binding to collagen [38, chapter 18]. Although platelet activation under flow conditions is an interesting topic for studies, our work presented in subsequent chapters focuses on the simpler study of platelet activation under static conditions.

${ }^{3}$ GPIb-IX-V is more important for platelet capture, forming rapid, but unstable bonds with VWF, whereas GPVI is the more potent initiator of internal signaling [38, chapter 18]. 


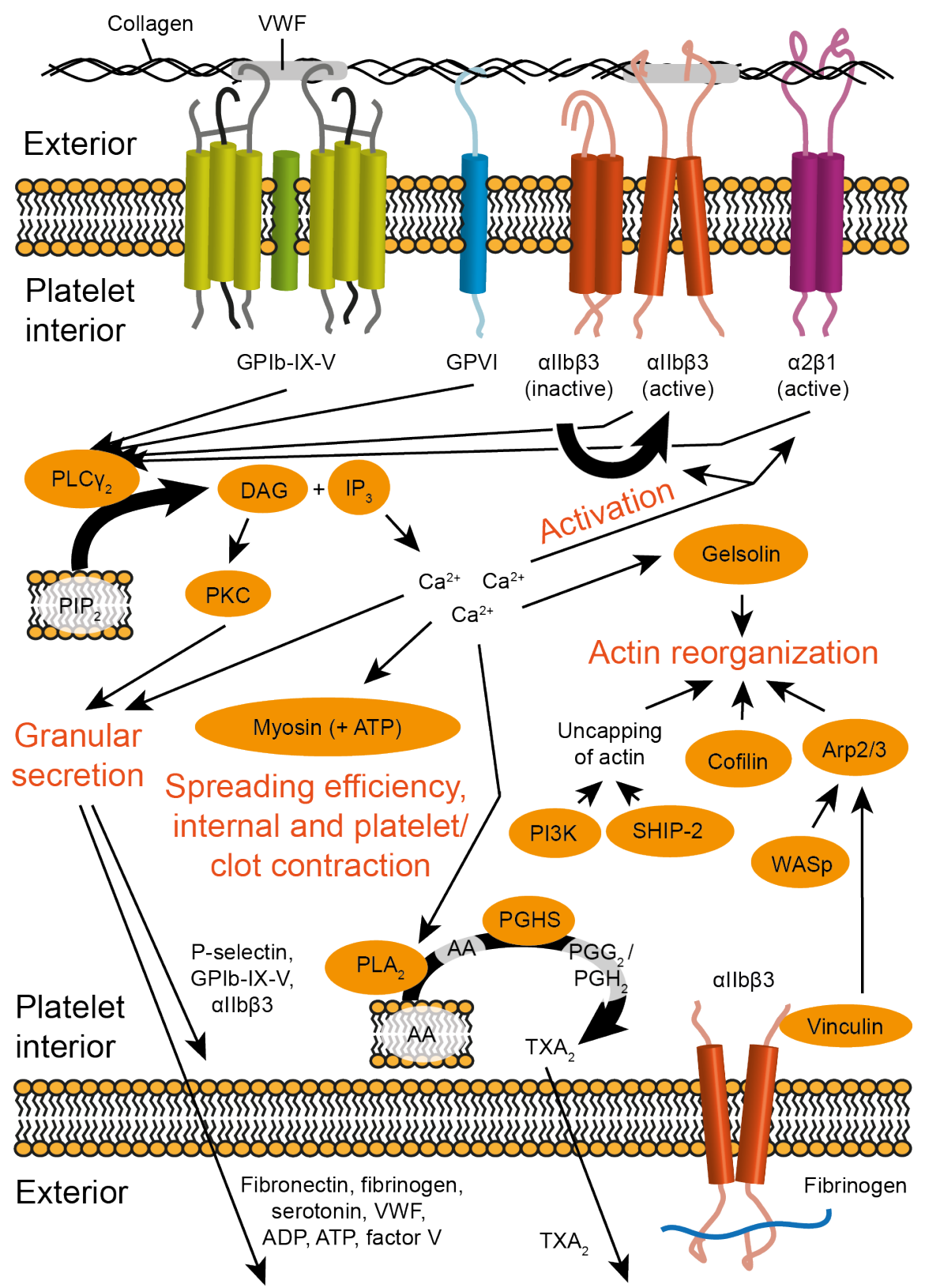

Amplification of platelet activity, platelet aggregation

Figure I.3: Regulatory signaling during platelet activation: Platelet adhesion to collagen or von Willebrand factor (VWF) via the membrane receptors GPVI, $\alpha 2 \beta 1$, GPIbIX-V and $\alpha \operatorname{Ib} \beta 3$ initiates a signaling cascade that regulates the different stages of platelet activation. Thus, adhesion is reinforced by activation of additional $\alpha \operatorname{IIb} \beta 3$ and $\alpha 2 \beta 1$ receptors. Platelet shape change and spreading through immobilized membrane receptors is accomplished by actin reorganization and myosin motor contractility. The same motors also mediate centralization of granules (internal contraction), which later fuse with the platelet membrane to release their content. This granular content in turn is recognized by other receptors on platelets in a feedback loop that amplifies internal signaling (see also figure I.7). Following amplification, platelets start to aggregate and finally contract to compress the clot mass via myosin motor activity. [Abbreviations listed on page viii) figure adapted from [38, p. 149, 169, 322, 336]] 
ing actin filaments as well as with the pointed end of actin filaments, it allows actin polymerization to proceed along entirely new directions and enables branching.

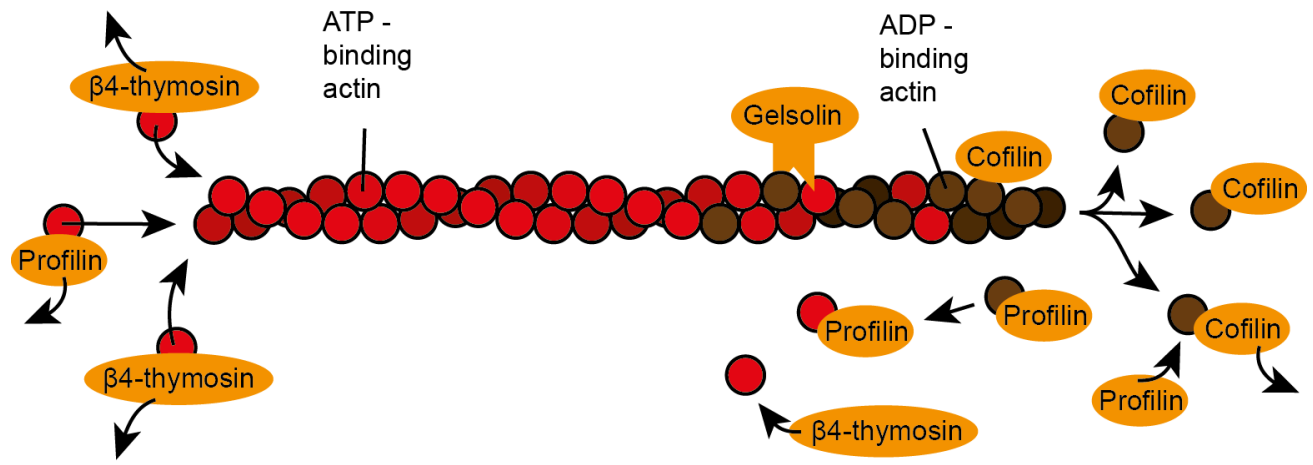

Barbed end

Pointed end

Figure I.4: Actin polymerization: After dissociation from profilin and $\beta 4$-thymosin, ATPbinding actin monomers are inserted at the barbed end of an existing actin filament. After a certain time, the bound ATP is hydrolyzed to ADP, so that ADP-binding actin subunits accumulate at the pointed end of the filament. ADP is then recognized by cofilin or the actin-severing gelsolin. Once cofilin attaches to an actin subunit, it becomes destabilized and subsequently dissociates from the filament. As soon as cofilin releases the actin monomer it can once more be bound by profilin, which additionally facilitates the exchange of ADP for ATP. ATP-binding monomers are also recognized by $\beta 4$-thymosin and the cycle begins anew.

During actin polymerization at the cell's periphery, ATP-binding actin monomers are incorporated into the existing actin fiber at the barbed end (figure I.4). To that end, they dissociate from $\beta 4$-thymosin or profilin, which are two molecules that effectively seize monomers from the pointed end to deposit them at the barbed end of the filament. Once deposited at the barbed end, the actin in the filament starts aging as it approaches the pointed end of the filament, the bound ATP being hydrolyzed to ADP. ADP in turn is recognized by gelsolin and by cofilin. While gelsolin binding severs actin filaments, the binding of cofilin destabilizes the actin subunits so that they dissociate from the filament at the low-affinity pointed end. Subsequently, cofilin detaches from the ADP-binding monomers, so that profilin can bind to them. Profilin, in turn sequesters the monomers, while simultaneously facilitating the ADP-ATP exchange. Monomers that have reassociated with ATP can then also be bound by $\beta 4$-thymosin, effectively closing the cycle. This assembly of actin at the barbed end and simultaneous disassembly at the pointed end is termed actin turnover. Platelets regulate the processes at both ends to drive actin dynamics that eventually lead to platelet shape change. For example, platelets mediate actin disassembly via gelsolin and cofilin, the latter of which is activated by dephosphorylation (figure I.3). Actin polymerization, in turn, is controlled by first uncapping or newly creating barbed ends of actin filaments to initiate polymerization. Polymerization is then eventually stalled by recapping barbed ends with CapZ. During recapping, the vasodilator-stimulated phosphoprotein (VASP) can have inhibitory effects leading to the formation of longer filaments, for example, in filopodia. In the final stage, however, $60 \%$ of CapZ is bound to actin and $80 \%$ of the total amount of platelet actin is in filamentous form [38, p. 86]. 


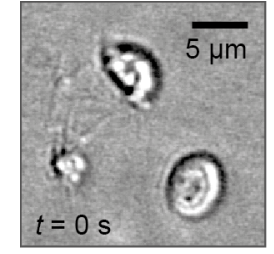

Adhesion
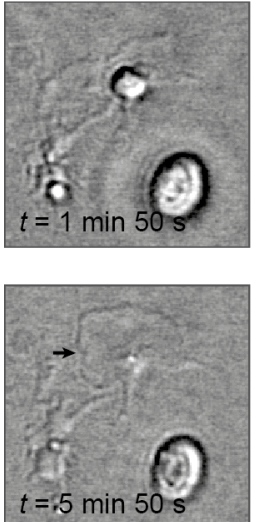
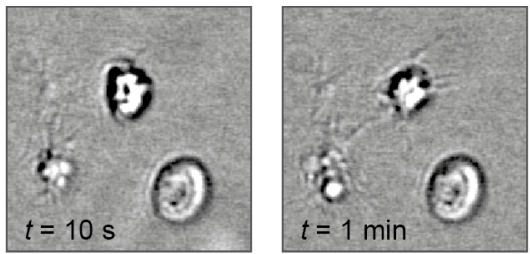

Increased filopod formation, platelet rounding
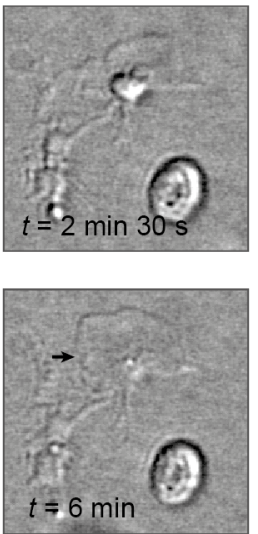

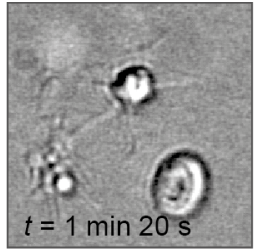

Extension of lamellipodium
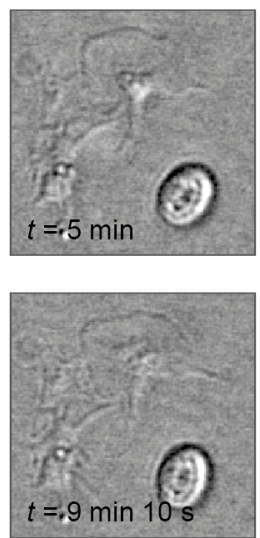

Formation of membrane rufles

Figure I.5: Platelet shape change during activation on glass: Snapshots taken from a movie of platelet activation on untreated glass cover slips. Platelets were kept in a humid atmosphere at $37^{\circ} \mathrm{C}$ throughout the experiment. For the movie, differential interference contrast (DIC) images were taken every $10 \mathrm{~s}$ with an inverted light microscope (60x objective). The images show different stages of activation including platelet adhesion (set as $t=0$ ), platelet rounding, extension of filopodia, formation of a lamellipodium and membrane ruffling (black arrows).

Actin polymerization generates protrusion forces (section I.2), allowing platelets to spread on the surface of a wound thereby increasing their surface area to up to $420 \%$ of its resting state [38, p. 68]. The additional membrane is probably recruited from the open canalicular system (OCS) of platelets. Experiments on glass surfaces show that spreading follows an initial rounding of the platelets [38, pp. 82] (see also figure I.5). It is initiated by the extension of several fingerlike filopodia that are lined with actin filaments reaching into the cell's center. Subsequently, broad and flat protrusions (lamellae) are generated containing a dense network of actin filaments that are $\sim 0.5 \mu \mathrm{m}$ in length. On glass, the membrane of platelets is found to be very dynamic even after spreading, displaying membrane ruffles that move inwardly and filopodia that are rotated around the cell contour. Platelet spreading is particularly efficient and fast, because the submembrane contractile system of the cell is able to move the cell surface through the rather immobile GPIb-IX-V and $\alpha \operatorname{Ib} \beta 3$ receptors that tether platelets to the wounded surface (figure [.3. see also [38, p. 50]). This submembrane contractile system is made up of actin in association with myosin motors. Outside of the submembrane region myosin motors also participate in internal contraction [62], a process that occurs simultaneously with platelet spreading (figure I.3). During internal 
contraction, the spreading platelet moves its granules and organelles into the center of the cell. Additionally, the partly intact microtubule coil is often compressed into the center, so that it finally surrounds the granules. The remaining microtubules that are not part of the coil, are found to radiate outwardly from the cell center and into newly formed lamellae and particularly into filopodia [38, p. 83].

\section{Platelet secretion and amplification of internal signaling}

Another effect that the increased $\mathrm{Ca}^{2+}$ levels in activated platelets trigger in combination with activated PKC is the secretion of granular content to the platelet exterior $[38$, p. 312][4] (figure I.3). To that end, granules fuse with the OCS at the cell center, their content travelling through the channels of the OCS to the extracellular environment. By these means clotting factors such as coagulation factor V, ADP, ATP, serotonin, VWF, fibronectin and fibrinogen are released. Furthermore, additional adhesive receptors such as P-selectin, GPIb-IX-V and $\alpha \operatorname{IIb} \beta 3$ from the granular membranes become available to the spreading platelet, increasing its tethering efficiency to both the wounded surface and in later stages to other platelets and cells.

At the same time as granular release is initiated, activated platelets synthesize and secrete the platelet agonist thromboxane $\mathrm{A}_{2}\left(\mathrm{TXA}_{2}\right.$ ) (figure I.3). $\mathrm{TXA}_{2}$ is produced as arachidonic acid is metabolized in activated platelets [38, p. 257 and pp. 565]. Arachidonic acid (AA), which is covalently bound to the plasma membrane in resting platelets, is cleaved from the membrane phospholipids by the enzyme phospholipase $\mathrm{A}_{2}$ as soon as calcium levels increase. Alternatively, p38 MAP kinase activity regulates AA release as part of the downsignaling cascade from GPIb-IX-V [38, p. 565]. In subsequent steps, prostaglandin $\mathrm{G}_{2}\left(\mathrm{PGG}_{2}\right)$ is formed from free AA in a reaction that is catalyzed by the enzyme complex prostaglandin endoperoxide $\mathrm{H}$ synthase (PGHS). This particular catalytic function of PGHS also gave it its alternate name cyclooxygenase (COX). However, the catalytic function of PGHS is twofold so that it also acts upon $\mathrm{PGG}_{2}$ in its hydroperoxidase (HOX) function catalyzing its two-electron reduction to the unstable $\mathrm{PGH}_{2}$. $\mathrm{PGH}_{2}$ is further converted into prostanoids such as $\mathrm{PGE}_{2}$, $\mathrm{PGD}_{2}, \mathrm{PGF}_{2}, \mathrm{PGI}_{2}$ and $\mathrm{TXA}_{2}$ in a tissue-specific manner. In platelets, in particular, thromboxane synthase is the catalyzer for the reaction from $\mathrm{PGH}_{2}$ to $\mathrm{TXA}_{2}[5]$.

A final soluble clotting factor that activated platelets help to generate is the protease thrombin [38, p. 51, 179 and pp. 377]. Thrombin is synthesized from blood circulating prothrombin by a complex at the plasma membrane of platelets (prothrombinase) consisting of coagulation factor $\mathrm{Xa}$ and Va (figure I.6). The prothrombinase complex, in turn, assembles on platelets by $\mathrm{Ca}^{2+}$ dependent interactions. One of its constituents, coagulation factor Va, is the activated form of coagulation factor V [38, pp. 381]. Fac-

\footnotetext{
${ }^{4}$ Gene-deletion studies have shown that PI3K is also involved in the signaling leading to granular secretion. It activates the protein Akt, the isoform Akt2 being related to granular secretion, although details on its effect are largely unknown [38, p. 328].

${ }^{5}$ Aspirin blocks COX both in platelets and in the endothelium, an effect that is irreversible at least for platelets. Interestingly, at low concentrations aspirin only affects COX-1 in platelets, whereas COX-2 in the endothelium is hardly influenced. Consequently, the extrusion of the platelet inhibitor $\mathrm{PGI}_{2}$ by the endothelium can proceed unhindered after the administration of low-dosage aspirin. Furthermore, the effect of aspirin is modulated by the shear stress prevalent at the site of platelet aggregation, meaning that elevated shear stresses can completely overcome aspirin inhibition.
} 
tor $\mathrm{V}$ is either recruited from the blood stream or it is released in combination with its activated form Va from platelet granules upon platelet activation. Interestingly, thrombin itself activates factor $\mathrm{V}$ to Va. The other constituent of prothrombinase, factor $\mathrm{Xa}$, is activated from blood circulating factor $\mathrm{X}[6]$ by the joint action of tissue factor and coagulation factor VIIa. While factor VIIa is already present in the blood stream, tissue factor only becomes available at a wounded site. Thus, upon blood vessel damage subendothelial tissue factor is exposed and some blood circulating microvesicles carrying tissue factor attach to P-selectin on the surface of activated platelets. Furthermore, platelets themselves expose tissue factor on the plasma membrane and possibly release it from internal microparticle and microvesicle stores following platelet activation.

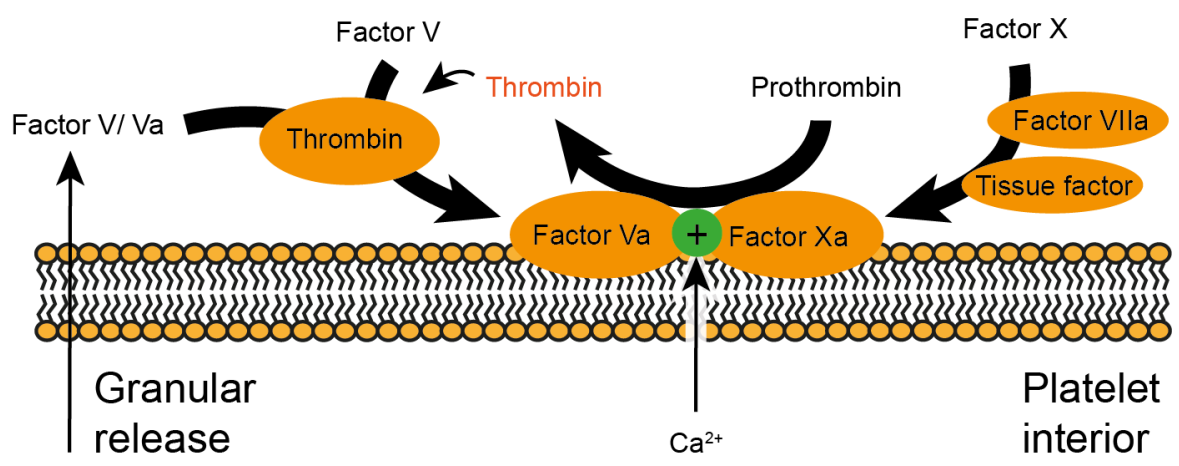

Figure I.6: Platelets accelerate thrombin generation: Upon blood vessel damage tissue factor becomes available at the site of injury. Tissue factor then acts in conjuncture with blood-circulating coagulation factor VIIa to convert soluble factor X into its active counterpart factor Xa. Simultaneously, blood-circulating or platelet-derived factor V is activated to factor Va. Factor Va and factor Xa can subsequently assemble into a complex in a $\mathrm{Ca}^{2+}$-dependent mechanism. This complex, also known as prothrombinase, finally converts soluble prothrombin into thrombin. Interestingly, thrombin itself is able to activate factor $\mathrm{V}$ to $\mathrm{Va}$ and this feedback response amplifies thrombin generation at the wounded surface.

Of all the soluble factors that become readily available at a wounded blood vessel surface, thrombin, ADP and $\mathrm{TXA}_{2}$, play an essential role in amplifying platelet activation [7] (figure I.7). ADP, in particular, is among the earliest available, being released not only by activated platelets, but also by damage to both tissue and red blood cells as a consequence of wound infliction [38, p. 325]. The three clotting factors are recognized by different receptors on platelets: ADP is recognized by $\mathrm{P}_{2} \mathrm{Y}_{1}$ and $\mathrm{P} 2 \mathrm{Y}_{12}$, $\mathrm{TXA}_{2}$ is recognized by TP and thrombin is recognized by the protease-activated receptors PAR-1 and PAR-4 ${ }^{[8]}$. All these receptors (GPCR) couple to GTP-binding proteins [38, pp. 323]. The GPCRs are, however, not specific to one particular family of GTPbinding proteins. Consequently, one and the same receptor can couple to GTP-binding proteins from different families [38, p. 321]. For example, PAR-1 can couple to a

\footnotetext{
${ }^{6}$ GPIb-IX-V promotes assembly of prothrombinase by binding and recruiting factor X from the blood stream to the platelet plasma membrane [38, p. 145].

7 On their own, all three clotting factors can also trigger platelet activation.

8 PAR-1 and PAR-4 get activated as they are cleaved by thrombin, a process that is probably facilitated by thrombin binding to GPIb-IX-V on the platelet surface [38, p. 333 and pp. 365].
} 
GTP-binding protein from either the $\mathrm{G}_{q}$ family, the $\mathrm{G}_{i}$ family or the $\mathrm{G}_{12}$ family. In fact, all three coupling configurations are probably found in vivo for PAR-1 receptors. Depending on the type of GTP-binding protein, the signaling cascade initiated by a GPCR occurs a little differently [38, chapter 16]. One pathway, for example, involving members of the $\mathrm{G}_{q}$ family leads to activation of $\mathrm{PLC} \beta$ downstream of ADP, thrombin or $\mathrm{TXA}_{2}$ binding. PLC $\beta$ then reinforces $\mathrm{Ca}^{2+}$ release in the cells and activates additional PKC by the same means as the previously mentioned PLC $\gamma$ (see page 7). Other pathways lead to the suppression of the inhibiting cyclic adenosine monophosphate (cAMP). Both amplification of the cytosolic $\mathrm{Ca}^{2+}$ and PKC levels and simultaneous suppression of cAMP are needed to sufficiently activate the platelet receptor $\alpha \operatorname{IIb} \beta 3$ to induce platelet aggregation [38, p. 326 and p. 365].

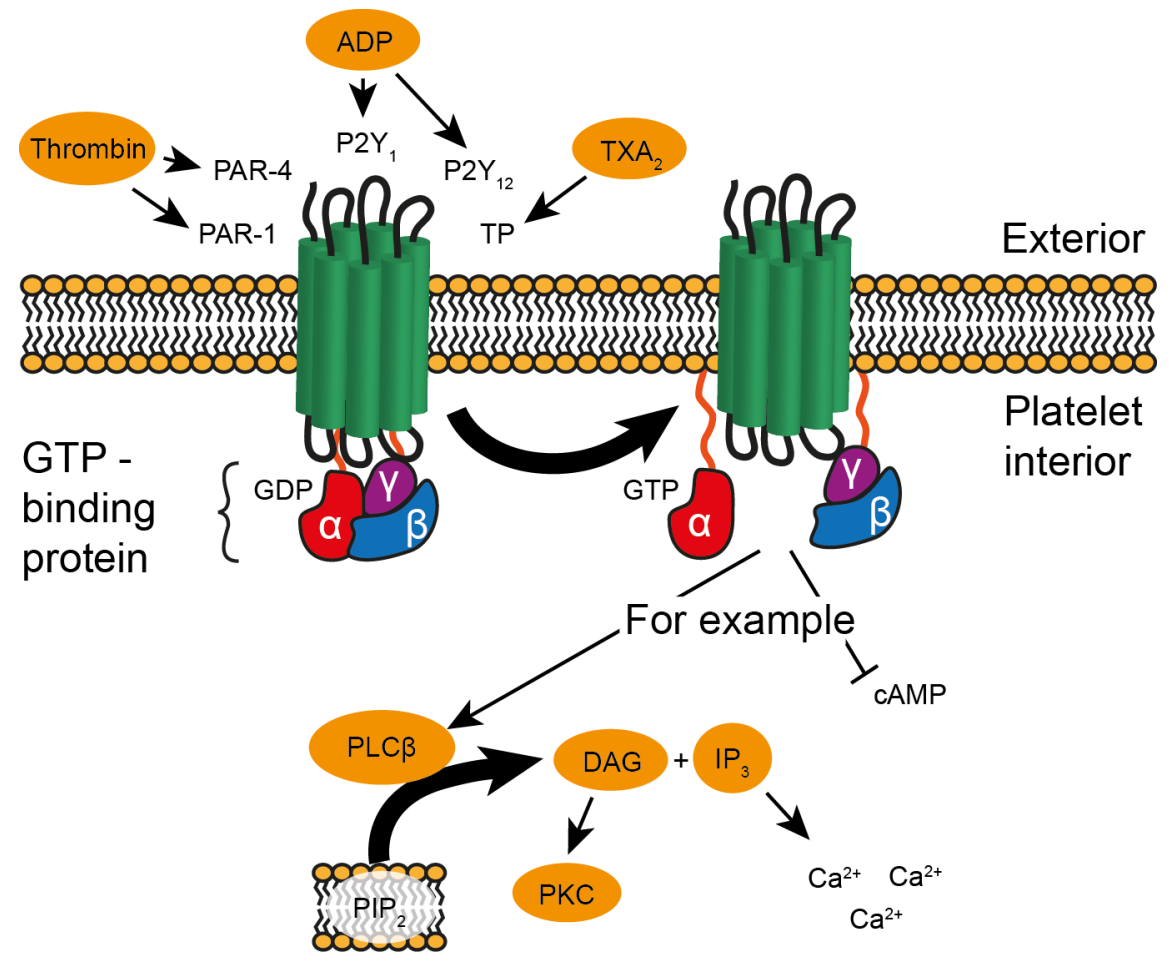

Figure I.7: Amplification of platelet activation: Activated platelets help to increase ADP, thromboxane $\mathrm{A}_{2}\left(\mathrm{TXA}_{2}\right)$ and thrombin levels at the site of blood vessel injury. These clotting factors are again recognized by receptors on the platelet surface that are coupled to GTP-binding proteins (G-proteins). Receptor binding in turn effectively amplifies platelet internal signaling. The exact signaling that occurs downstream of receptor binding depends both on the recognized clotting factor and the type of G-protein that is coupled to the receptor. One positive feedback response that all three clotting factors can trigger is the rise in internal $\mathrm{Ca}^{2+}$ and protein kinase $\mathrm{C}$ (PKC) levels that occurs downstream of phospholipase $\mathrm{C} \beta$ (PLC $\beta)$ activation. Both $\mathrm{Ca}^{2+}$ and $\mathrm{PKC}$ are needed to sufficiently activate the platelet receptor $\alpha \operatorname{IIb} \beta 3$ that mediates platelet aggregation. This positive feedback is complemented by a negative feedback response that can occur downstream of ADP binding. Here, the generation of platelet inhibiting cyclic adenosine monophosphate (cAMP) is eventually suppressed. Additional abbreviations: $\mathrm{PIP}_{2}$ : phosphatidylinositol 4,5 biphosphate; DAG: diacylglycerol; $\mathrm{IP}_{3}$ : inositol triphosphate [Design based on figures in [38, p. 323, 326, 329]] 


\section{Platelet aggregation}

During blood clotting, activated platelets covering the wound surface start to recruit additional cells to form a platelet aggregate [38, p. 75]. Many of the mechanisms described in the previous sections are essential to achieve this goal. Activated platelets use their newly extended filopodia to attach to new platelets. They also facilitate intracellular adhesion by recruiting adhesive P-selectin receptors and additional $\alpha \operatorname{IIb} \beta 3$ receptors to their outer surface via granular fusion with the plasma membrane (figure I.3). Furthermore, they activate the $\alpha \operatorname{Ilb} \beta 3$ receptors at the plasma membrane, while also releasing platelet attractants such as ADP, serotonin and $\mathrm{TXA}_{2}$ or accelerating the generation of the platelet agonist thrombin (figure I.2 b)).

Platelet aggregation is then thought to proceed in two phases [38, chapter 18]. In an initial phase, VWF bonds are established between platelets using the GPIb-IX-V (figure I.2 b)). To enhance this initial binding, activated platelets are known to secrete the largest VWF multimers via granular release, which form the tightest bonds with GPIb-IX-V. The advantage of first establishing VWF bonds, is that these form rather rapidly thus keeping platelets in close proximity to each other in spite of the high shear stresses prevailing in blood flow. This in turn allows the more stable fibrinogen bonds and possibly also fibronectin bonds to subsequently form across $\alpha \operatorname{IIb} \beta 3$ receptors [38, pp. 367]. Fibrinogen bound $\alpha \operatorname{Ibb} \beta 3$ in platelets is attached to the underlying cytoskeletal actin network by a number of proteins forming focal adhesion sites, including talin, vinculin, and $\alpha$-actinin [38, p. 88, pp. 170 and p. 352].

As the platelet-fibrinogen aggregate grows other cells such as red blood cells are incorporated into the mesh (figure I.2 b)), red bloods perhaps even serving a more active role in promoting clot formation [1].

\section{Clot contraction}

A final effect that increased $\mathrm{Ca}^{2+}$ levels have on platelets is that contraction of the actin-bound myosin motor is triggered [38, p. 88] (see also figure [.3). Thus, myosin activity in the submembrane actin network moves the plasma membrane through the largely immobile membrane receptors during initial platelet spreading. Further on in the platelet activation process, myosin contraction in conjuncture with cytosolic actin pulls granules and organelles into the cell center and finally myosin activity acts on each platelet to contract and solidify the clot mass. As the rise in $\mathrm{Ca}^{2+}$ concentrations occurs early on during platelet activation, platelet contraction can actually begin even before actin reorganization is complete. In the following paragraph a brief review will be given of myosin structure, its assembly and its abundance in platelets. Myosin activity is explained in more detail in section I.2.

Myosin is found in platelets in form of non-muscle myosin IIA and IIB [9] [38, p. 88]. Myosin II consists of two extended 220-kDa heavy chains with a head, neck and tail region [34, p. 769]. At the neck region of the heavy chain, myosin has two associated 20-kDa light chains and two associated 15-kDa light chain. By phosphorylation of the 20-kDa light chains (MLC), myosin undergoes a conformational change,

\footnotetext{
${ }^{9}$ It is, nevertheless, likely that other non-filamentous forms of myosin coexist in platelets along with non-muscle myosin IIA and IIB.
} 
which enables it to assemble into short filaments via the myosin tail region (figure I.8). These filaments contain 28 molecules, are about $300 \mathrm{~nm}$ in length and are bipolar, the myosin heads being localized to both ends [38, 54, p. 88]. The phosphorylation leading to myosin filament assembly can be accomplished by two agents: either by myosin light-chain kinase (MLCK) or by rho kinase (ROCK) [38, 16, p. 88]. While MLCK is activated through calcium and calmodulin, ROCK is bound and activated by rho A, a molecule that in turn is activated downstream of thrombin or $\mathrm{TXA}_{2}$ signaling [38, p. 327]. Apart from promoting MLC phosphorylation directly, ROCK further supports myosin filament assembly by inhibiting the counteracting MLC phosphatase. After phosphorylation of the MLC, myosin filaments associate with actin filaments. Having associated with actin filaments in an actin bundle, myosin can move parallel, neighboring filaments along each other under consumption of ATP. By inducing this filament sliding myosin effectively compresses the actin bundle.

\section{Clot stabilization and regulatory signals}

In the final stages of clot formation, several mechanisms come into play that stabilize the clot mass. Among these, is the thrombin-mediated conversion of plateletinterconnecting fibrinogen into insoluble fibrin strands [38, p. 179 and p. 421]. Fibrinogen is a protein comprised of two sets of $\mathrm{A} \alpha, \mathrm{B} \beta$ and $\gamma$ subunits [38, p. 421]. It is symmetrically arranged with two lateral $\mathrm{D}$ domains and a central $\mathrm{E}$ domain. Contained in the D domain is the $\gamma$ residue consisting of the amino acids Lys-Gln-AlaGly-Asp-Val (KQAGDV) that is primarily involved in platelet-fibrin(ogen) binding $[10]$ [38, p. 168]. During conversion to fibrin, thrombin cleaves fibrinogen at Arg-Gly both in the $\mathrm{A} \alpha$ and the $\mathrm{B} \beta$ chain [38, p. 179], thereby exposing new binding sites in the central E domain. Other fibrin(ogen) molecules can then attach to these binding sites via their D domains, thus allowing fibrin polymerization to proceed [60].

Fibrin formation, however, is only one of the mechanisms that enforce clot stability by strengthening platelet-platelet bonds [38, p. 369]. Thus, for example, the CD40 ligand is able to support inter-platelet connection by interacting with $\alpha \operatorname{Ilb} \beta 3$. Again other mechanisms leading to clot stability aim at keeping platelet internal signaling active, so that the platelet receptor $\alpha \operatorname{IIb} \beta 3$ remains in its active conformation [38, p. 369]. For that purpose, newly aggregated platelets send out feedback signals to the underlying, activated platelets, which are probably recognized by the ADP receptors $\mathrm{P}_{2} \mathrm{Y}_{1}$ and $\mathrm{P}_{2} \mathrm{Y}_{12}$. The effect of these feedback signals can be observed as oscillations in the calcium concentration in activated platelets. Likewise, the hormone leptin seems to generate signals that enhance and sustain platelet activation. Also, Eph kinases and ephrins contribute to signaling induced by bound $\alpha \operatorname{IIb} \beta 3$ (outside-in signaling), which further supports clot stability. These are just a few factors among many that contribute to clot stability.

At the same time as the clot is stabilized, regulatory signals control clot size and confine the clot to the site of injury. Ranging in the first category is the platelet antagonist nitric oxide (NO). NO is not only secreted by the endothelium (see also page 5),

\footnotetext{
10 Technically, fibrinogen possesses secondary binding sites for inter-platelet connections in the form of the two Arg-Gly-Asp (RGD) motifs on the A $\alpha$ chains. It appears, however, that these two bindings sites play only a minor role, if they are used at all [38, p. 168].
} 


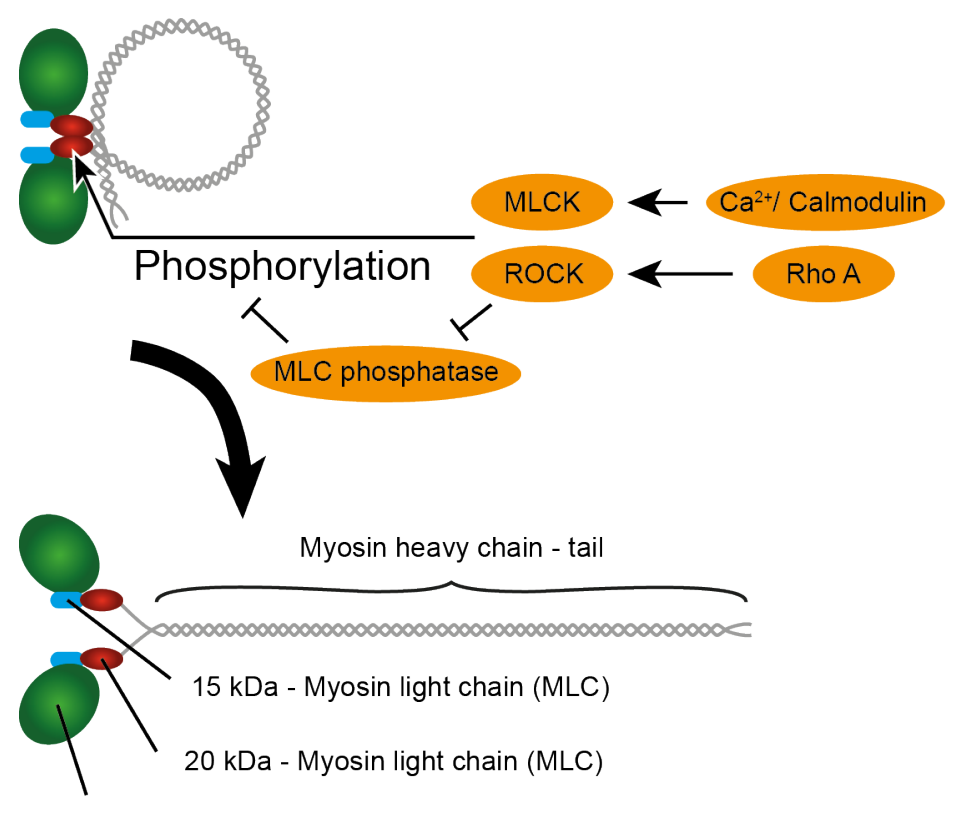

Myosin heavy chain - head
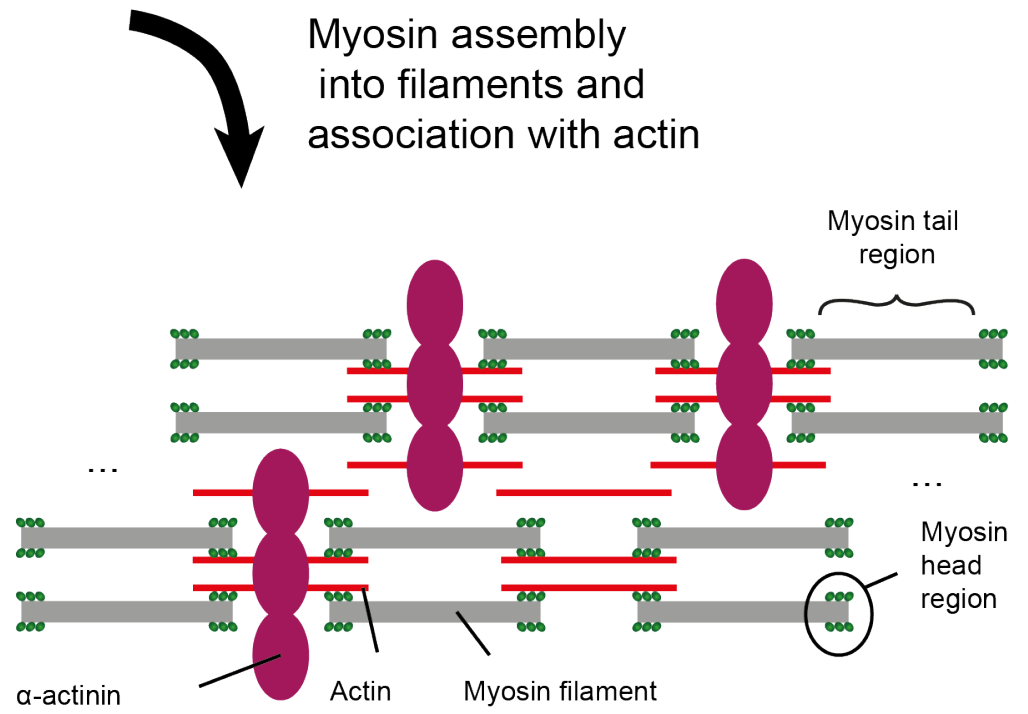

Figure I.8: Assembly of non-muscle myosin IIA filaments and association with the platelet actin network: During platelet activation, cytosolic $\mathrm{Ca}^{2+}$ levels are increased, which leads to the activation of myosin light chain kinase (MLCK). Additionally, platelets activate rho A, which again activates rho kinase (ROCK). Both ROCK and MLCK then regulate phosphorylation of the $20 \mathrm{kDa}$ - myosin light chain (MLC). ROCK further promotes this phosphorylation by inhibiting MLC phosphatase. The phosphorylation then brings about a conformational change in the myosin IIa molecule. Following this conformational change, myosin motors are able to assemble into short filaments, which subsequently associate with the platelet actin network to form contractile stress fibers. [Design adapted from figures in [54] and [16] 
but it is also released in elevated concentrations by activated platelets themselves to control aggregation and prevent the clot from getting too large [38, p. 254]. Furthermore, the ADP and ATP that becomes available through platelet secretion during clot formation, promotes the release of both $\mathrm{NO}$ and the inhibiting $\mathrm{PGI}_{2}$ (see page 5 ) by endothelial cells, thus reinforcing the regulation of clot size [38, p. 264]. Endothelial cells lining the wound edge play even a further role: the glycoprotein CD39 (or NTPDase1) in their plasma membrane converts the platelet agonist ADP (and ATP) into adenosine monophosphate (AMP) [38, p. 262]. AMP is then further transformed by CD73 into adenosine, which is in itself a platelet inhibitor [38, p. 252]. This regulation of clot size is complemented by molecules that prevent disintegrated parts of the clot from spreading through blood circulation. An example of these, is the protease ADAMTS-13 [38, p. 368]. It cleaves circulating VWF multimers, thus reducing their size and binding efficiency.

\section{Clot formation in arterial and venous circulation}

Clot formation occurs a little differently in arteries and veins [38, chapter 16]. The description of the process that is given in the previous sections only applies if the injury is to arterial blood vessels. In arteries the blood flow velocities and related shear stresses are rather high and platelets become important agents during hemostasis. Thus, at the surface of activated platelets thrombin generation is accelerated. Additionally, platelets represent a physical barrier so that soluble agonists are not as easily washed away. Moreover, platelets contribute to the pool of agonists themselves by releasing additional clotting agents. Platelets also provide a base for fibrin network formation, a process that would otherwise be hampered by the high shear stresses in the blood flow. In contrast, venous circulation is characterized by comparatively low flow rates and related shear stresses, so that clotting factors can easily accumulate at the site of injury. Here, clots are essentially composed of trapped red blood cells containing only few platelets.

\section{I.2 Contractile Forces in Cells}

In the previous sections, we introduced platelets as an example of contractile cells and highlighted the importance of platelet contraction during blood clot formation. However, platelets are usually not the first cells to be associated with cellular contraction. In fact, cellular contraction is most often associated with muscle cells. Yet, muscle movement and clot formation are not the only processes that are regulated by contractile forces. Cell locomotion is another example of a process, in which cellular contractile forces play an important role. In the following section, we will therefore review some of the cell types that generate contractile forces and point out both the differences between them and common traits that they share.

As already mentioned, among the most prominent cells generating contractile forces are muscle cells [34. Muscle cells can be subdivided into three categories: skeletal, smooth and cardiac. Because cardiac muscle cells are rather special, displaying continuous and oscillatory contraction, they will not feature in the following section. The 
other two cell types contract spontaneously, each cell type serving a different purpose. As the name indicates, skeletal muscles are connected to the bones and their contraction is related to conscious, voluntary and reflexive movements of our body. Alternatively, skeletal muscles are used to create tension, for example when we hold an object above ground. In contrast, smooth muscle cells are associated with internal organs and their contraction regulates unconscious processes. These include, for example, blood vessel constriction or dilation and the movement of food through our digestive system. To accomplish the various tasks efficiently, skeletal muscle cells contract quickly, while the slower smooth muscle cells generate the larger forces [20]. Despite these differences, both cell types use the same cellular components to generate contractile forces: myosin II motors in combination with cytoskeletal actin. These two components are just the same as the ones that platelet contraction relies on.

In fact, myosin II and actin form the basis for the most prominent contractile mechanisms in cells. Locomoting cells such as fibroblasts and fish keratocytes also use acto-myosin contraction to assist propulsion ([34, pp. 787], 41]). Here, the cells initially extend filopodia and lamellipodia in the direction of motion and quickly anchor the new extensions to the substrate by forming tightly adhering focal adhesions. Subsequently the adhesions at the back are removed and the rearward membrane is retracted to create propulsion. In this last step acto-myosin contraction is thought to play an important role, although it is still unclear to which degree it is involved in the process. Some experiments on locomoting amoebae cells have shown that acto-myosin is not essential to cell locomotion and that cells can move even if myosin activity is lacking 61. However, in these studies it was noticed that acto-myosin activity considerably speeds up cell movement. Also, both fish keratocytes and fibroblasts display organized patches of myosin. In fish keratocytes there is a band of myosin between the lamellipodium and the cell body running in a line perpendicular to the cell motion [34, p. 789]. In fibroblasts, in turn, acto-myosin organizes into distinct stress fibers running the length of the cell in a direction parallel to the cell motion [34, p. 753]. Both the myosin band and the stress fibers are ideally positioned to generate and transmit contractile forces in the direction of cellular motion. Furthermore, laser cutting of stress fibers has demonstrated their capacity to contract [34, p. 784]. Nevertheless, it was suggested that the primary function of at least stress fibers is related to cell adhesion. Experimental evidence that is most indicative of the fact is twofold [34, p. 784]. First, fibroblasts display only few stress fibers during fast locomotion, their number increasing considerably when cells stop moving. Secondly, stress fibers are rarely formed in tissue cells, becoming only prominent in cells that are cultured on artificial glass or plastic surfaces. However, even if stress fibers do not generate contractile forces that lead to cell propulsion, there is an acto-myosin network in the cell apart from the stress fibers [34, p. 752] that can perform this task. The debate concerning acto-myosin involvement in cell locomotion is ongoing. Nevertheless, it can be stated that acto-myosin contraction plays at least an assisting role in generating cellular propulsion.

Having established that muscle cells, platelets and migrating cells all use the same building blocks to generate contractile forces, the question naturally arises how these building blocks can lead to the differences in cell behavior. How, for example, can 
a)

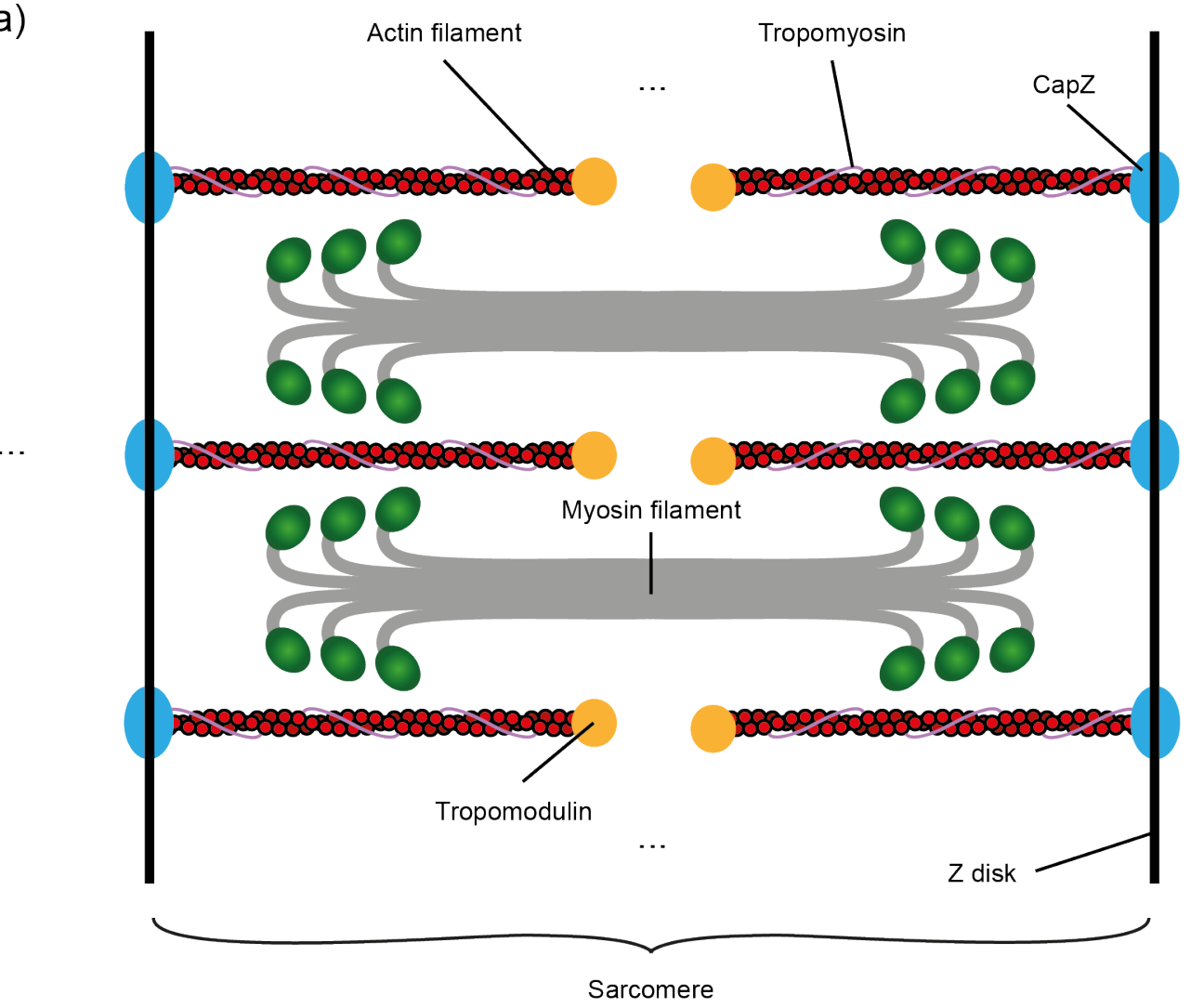

b)

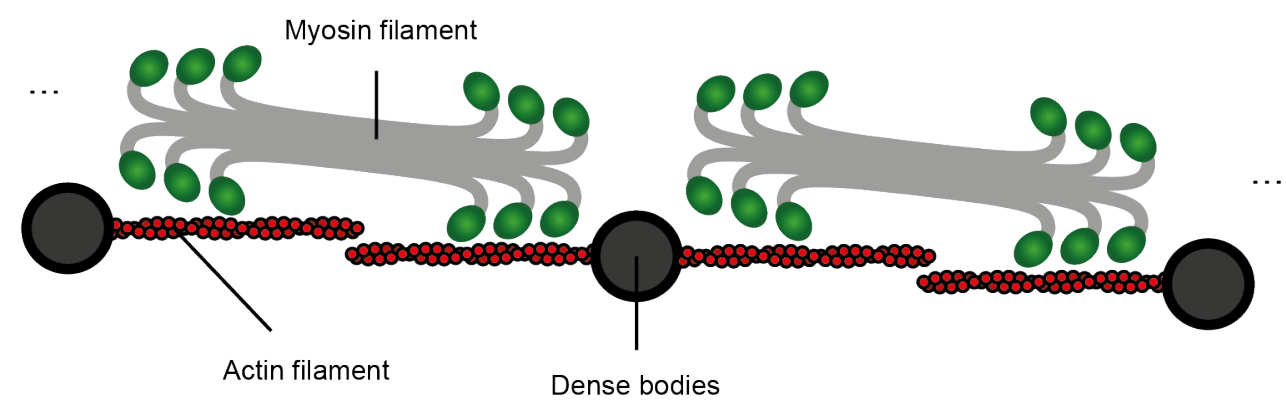

Figure I.9: Acto-myosin structures in muscle cells: The smallest working unit of a skeletal muscle is a sarcomere (a). A sarcomere is composed of an array of adjacent, antiparallel actin filaments that are interspersed by myosin filaments. Each sarcomere unit is bordered by the so called $\mathrm{Z}$ disk, into which the actin filaments are anchored via the capping protein CapZ. Furthermore, both CapZ and tropomodulin stabilize the actin filaments by preventing depolymerization from the filament ends. Coiled around the actin filaments is tropomyosin which regulates actin-myosin interaction. In contrast to the strict organization in skeletal muscles, actin and myosin are only loosely bundled in smooth muscle cells (b). Here, the dense bodies that actin filaments bind to serve similar functions as the $\mathrm{Z}$ disk in skeletal muscle cells. [Figure based on reference [34, p. 775, 777 and 780]] 
acto-myosin contract quickly for skeletal muscles and more slowly, yet more strongly for smooth muscle cells? How can it have a fixed direction of action in skeletal and smooth muscle cells, while keeping a flexible contractile axis in migrating cells that sometimes change their direction of motion? Finally, what is the directionality and speed of action in platelet contraction and what are the underlying, regulating mechanisms there? The last of these points represents the question that this work aims to address, while the former points have already been extensively studied. It was, for example, found that the speed of action and the directionality are related to the geometrical organization and assembly of acto-myosin structures. Thus, acto-myosin in migrating cells has a flexible direction of action, because it is assembled and disassembled into networks as the need arises. In contrast, acto-myosin structures in muscles are stabilized by proteins and therefore remain fixed [34, chapter 18]. Here, skeletal muscles display a strictly ordered array of myosin and actin filaments in so called sarcomeres, which are the smallest working unit of a skeletal muscle cell (figure I.9 a)). Every actin or myosin associated protein has its designated place in this array close to its point of action. Consequently contractile response can occur quickly. Contrary to the strict order in skeletal muscles, acto-myosin filaments in smooth muscle cells are only loosely bundled together, accounting to some extent for the slower response of this muscle type (figure I.9 b)). Another factor that slows down smooth muscle response is the slow rise of contraction-triggering $\mathrm{Ca}^{2+}$ levels in smooth muscle cells as compared to skeletal muscle cells [34, p. 781]. Also, some of the actin-regulating proteins and much of the signaling cascade leading to muscle contraction is different for the two muscle types [34, chapter 18]. Final points to be considered when explaining the different responses of the two muscle cells, are the myosin-inherent properties [20]. Although both smooth and skeletal muscle myosins produce similar forces and have similar motor step sizes, the two forms of myosin II have different actin-interaction times. Overall, myosin II from smooth muscle cells interacts with actin to generate forces for a longer time period than skeletal muscle myosin II. To better understand this point and introduce some quantitative measures that can be compared, we will subsequently give a brief review of myosin activity in association with actin (figure I.10, see also reference [34, pp. 769]).

Before myosin interacts with actin it binds an ATP molecule at the back of one of the myosin heads. This opens a cleft in the myosin head running from the back to the tip of the head. When ATP is hydrolyzed to ADP the cleft is partially closed, the head rotates about the neck and then binds to an actin filament. After binding to actin, one of the hydrolyzation products, the phosphate $\mathrm{P}_{i}$, is released at the back of the myosin head and the myosin head rotates back thereby pulling on the actin filament (power stroke). Having completed the power stroke the second hydrolyzation product, ADP, is released and myosin remains in a state tightly bound to actin. If ATP is again bound to myosin, the renewed opening of the myosin cleft destabilizes the myosin-actin bond and myosin dissociates once more from the actin filament. The myosin activity cycle can then start anew (figure I.10). It should be noted that the process described above for the actin-myosin interaction is actually only a model for myosin activity, the so called cross-bridge cycle [34, p. 778]. In fact, it is still unclear whether each hydrolyzed ATP molecule leads to a single myosin step [34, p. 771]. Independently of the amount of ATP hydrolyzed, however, we can define a quantitative measure for 


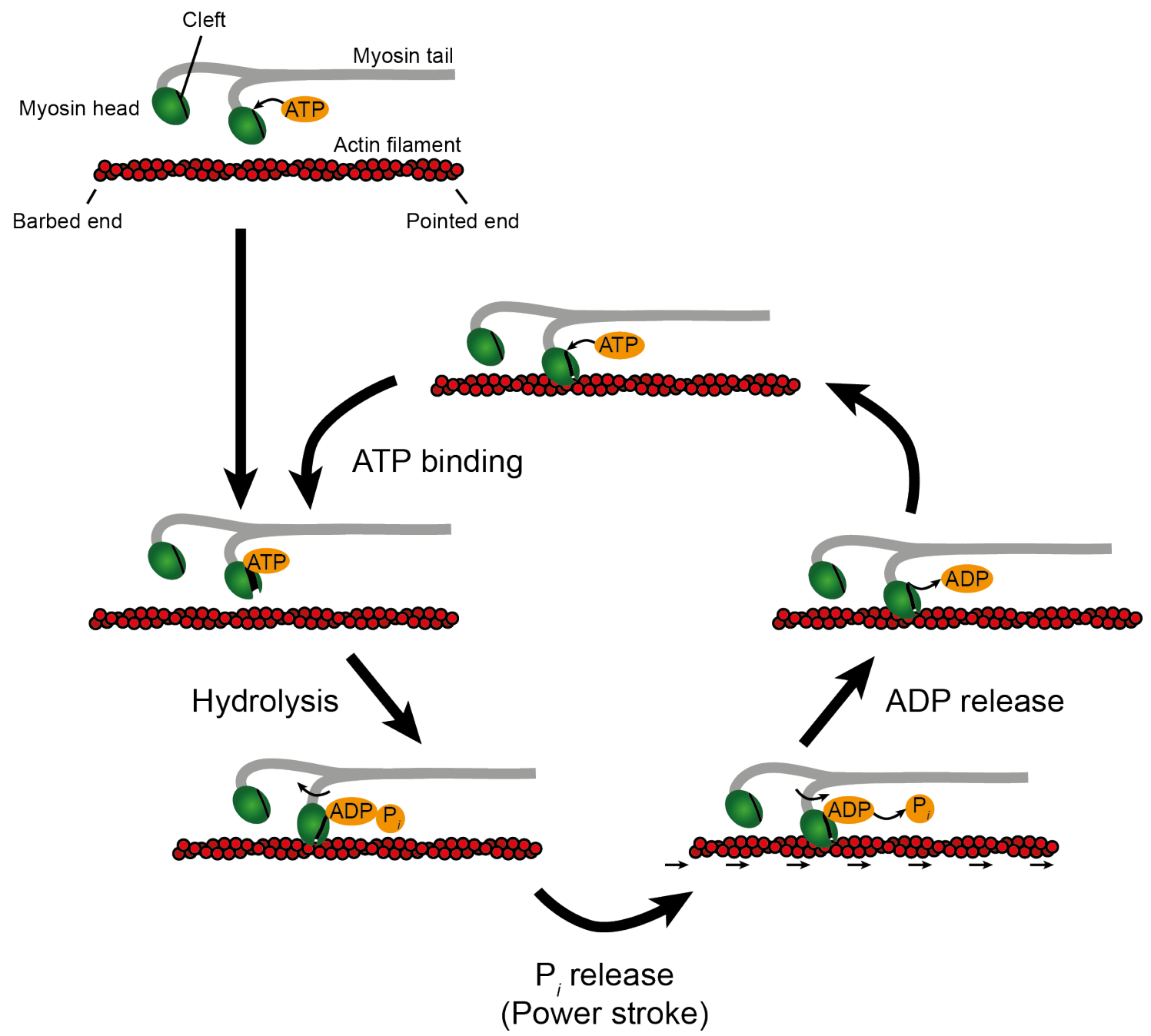

Figure I.10: Sketch of the working cycle of myosin (cross-bridge cycle): When myosin binds an ATP molecule this opens a cleft in the myosin head. Following ATP hydrolysis the cleft closes partially, the myosin head rotates about its neck and binds to a subunit in an actin filament. Subsequently, the hydrolysis products, ATP and phosphate $\mathrm{P}_{i}$, dissociate from myosin inducing the myosin head to rotate again. As the myosin head still binds to the actin filament in this last pivoting act, the actin filament is pulled along with the motion of the myosin head. Having completed its power stroke, myosin remains tightly bound to actin until another ATP molecule induces myosin to dissociate from the actin filament to start the cycle anew. [Figure based on reference [34, p. 774]] 
the motor efficiency during a myosin cycle, the so called duty ratio. The duty ratio is the ratio between the time that myosin prevails in the strongly bound configuration with actin and the total duration of a myosin cycle [51, p. 531]. It is also indicative of the fraction of myosin heads that is simultaneously bound to actin at one time point. For non-muscle myosin IIA, for example, the duty ratio is 0.05-0.11 [27], which is in the same range as the duty ratio for skeletal muscle myosin (0.025) [51, p. 531]. Both are, however, lower than the duty ratio for either smooth muscle cells $(0.2-0.5)$ [51, p. 531] or non-muscle myosin IIB (0.23-0.4) [57]. This is one of the reasons that smooth muscle contraction is slow, but rather strong. Nevertheless all four types of myosin motors have a duty ratio that is small enough so that in general the two myosin heads do not bind simultaneously to actin [51, p. 531]. That is, the myosin II motors are non-processive and an ensemble of myosin motors is needed to keep moving an actin filament.

For this reason, myosin II motors bundle into bipolar filaments, in which the end regions of the filaments contain the myosin heads. To contract the cytoskeleton, each end of a myosin filament acts on one of two adjacent and anti-parallel actin filaments [34, pp. 777]. Myosin II motors always walk toward the barbed ends of actin filaments during a cross-bridge cycle, so that the proximal ends of the two actin filaments need to be pointed ends. As soon as the myosin heads start acting on the actin filaments, the two actin filaments get pulled together and start to slide along each other. By these means an actin bundle consisting of several actin filaments can be compressed (sliding-filament model; figure I.11). During bundle contraction, each single myosin motor can contribute forces of 1.3-3.5 pN [56, 40, 17, 20]. In some single myosin motor experiments even forces as high as 6 or $7 \mathrm{pN}$ were measured [46, 17].

The primary generation of contractile forces by myosin motors is complemented in vivo by a few minor force generating mechanisms. Among these is the force generated by actin polymerization or depolymerization. According to the Brownian ratchet model and its derivatives [45, 39], actin filaments can polymerize against the plasma membrane of a cell. To that effect, actin monomers squeeze into the gaps that thermal fluctuations generate between the membrane and the existing filament. This process then generates protrusion forces that push the cell membrane outwardly. By analogy, the reverse process of actin depolymerization generates contractile forces that pull the cell membrane inwardly. Both protrusion and contractile forces that were generated by actin filaments have experimentally been measured to be between $0.125 \mathrm{pN}$ and $5 \mathrm{pN}[18,15]$.

Another contribution to cellular forces arises from passive properties of the cell. In particular in adherent cells that spread on a surface, the shape change accompanying spreading is counteracted by passive elastic forces that the cell exerts as a whole. For a better understanding of these elastic forces, it is necessary to study the elastic properties of cells. One such study, for example, has been performed by Radmacher et al. 48] using atomic force microscopy (AFM) on platelets that were activated on glass. The study reveals that different regions in platelets have also different elasticities, which is characterized by the Young's modulus $E$ (see also section II.1). For the cell center Radmacher et al. measure values for $E$ of $1.5-4 \mathrm{kPa}$, for the adjacent inner filamentous region they measure values of $4-10 \mathrm{kPa}$. Surrounding the inner filamentous 

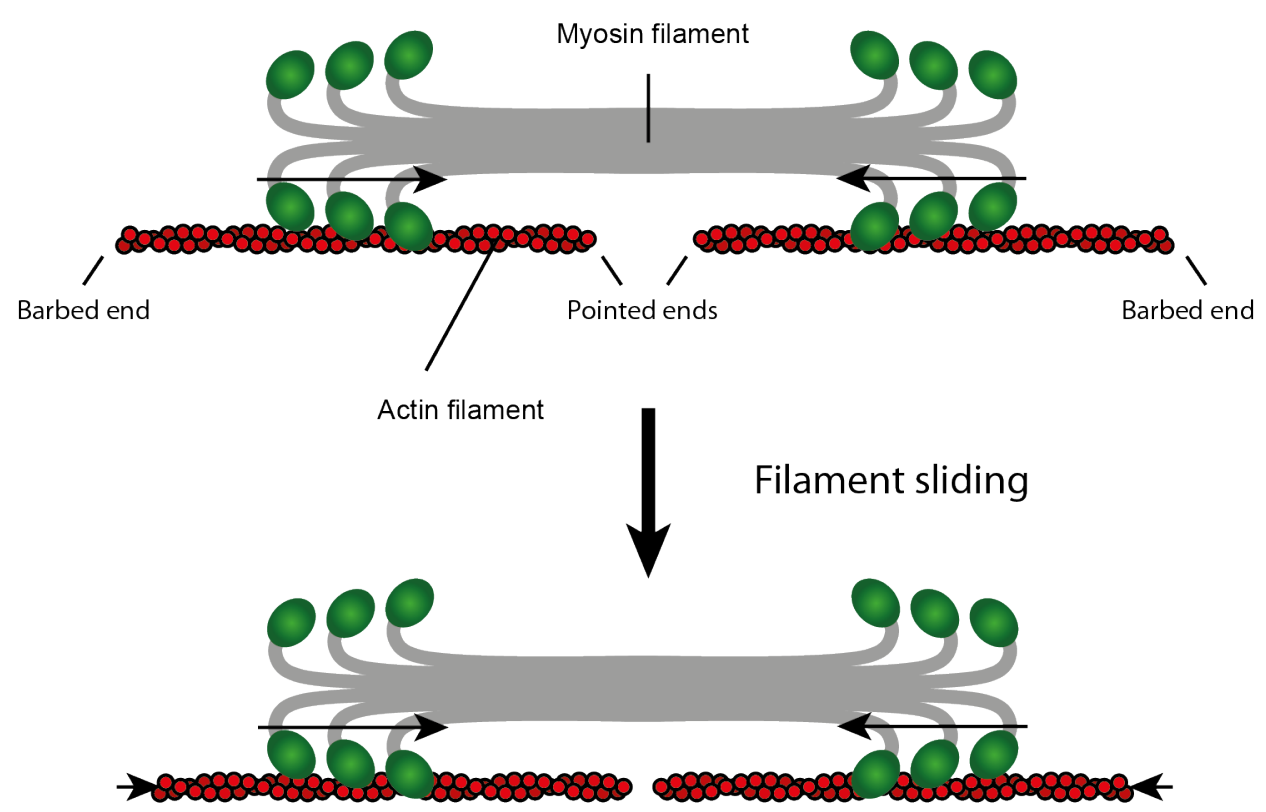

Figure I.11: Sliding filament model for actin bundle contraction: A bipolar myosin filament consisting of several myosin motors can align with actin filaments in an actin bundle. If the myosin heads at each end of the myosin filament act on a different actin filament and the two actin filaments have opposite orientations, myosin activity can pull both filaments toward each other. For sufficiently long actin-myosin interactions, the filaments eventually start to slide along each other, thereby also compressing the entire actin bundle. [Figure based on reference [34, p. 777]]

region they furthermore distinguish an outer filamentous region with elasticities of $10-40 \mathrm{kPa}$. Finally, they measure values for $E$ of $40-50 \mathrm{kPa}$ for the outermost cortex region of the platelet. As the authors of the paper, however, acknowledge, AFM tends to overestimate elasticities, if the measured sample is too thin. In such cases, the probing AFM cantilever is also sensitive to the underlying, significantly stiffer glass substrate. This effect is well known and models have recently been developed to correct for finite sample thickness [19, 13]. In their paper Radmacher et al. try to reduce the error due to finite sample thickness by diminishing the indentation amplitude of the AFM cantilever. Yet, it is still plausible that the larger elasticities that they measure for the thinner, outer regions of the platelet are overestimates. A value of $E=4 \mathrm{kPa}$, which has been measured for the thicker regions in the inner filamentous zone, may therefore be the best representative of the average platelet elasticity. A second elastic property, the Poisson ratio $\nu$, has been assumed to be 0.5 in the study by Radmacher et al (see also section II.1). Using both the average value for $E$ and the estimate for $\nu$, it is possible to estimate elastic forces for the shape changes that platelets undergo during spreading (see section VI.3).

Many if not all of the above mentioned force generating mechanisms will contribute to any force measurement that is performed on cells. Typically, such force measurements on soft substrates yield forces per cell area segment of, for example, $0.8-3.03 \mathrm{kPa}$ for fibroblasts [11, 41, 49] or $\mathcal{O}(0.1) \mathrm{kPa}$ for both fish keratocytes and airway smooth 
muscle cells [44, 7, 55]. The particular case of force measurements on platelets will be presented in more detail in the following section.

\section{I.3 Platelet Contraction}

Due to their medical importance, research on platelet contraction is traditionally closely linked to clinical applications. Thus, one of the commercially available instruments (Hemodyne ${ }^{\circledR}$ hemostasis analyzer; Hemodyne Inc., Richmond, VA - USA) that is used to measure platelet contractile forces was developed as a clinical measure to test platelet function [8, 10, 9]. Here, blood samples are placed in a conical cup, which is covered by a fitting conical lid (figure I.12 a)). Attached to the lid is a sensor that measures the vertical displacement of the lid, as the blood sample begins to contract. By periodically calibrating the device during one measurement with an externally applied compressive force, the displacement of the lid can be converted into force measures. Incidentally, the periodic calibration of the device yields information on the clot elastic modulus. By monitoring the platelet count in the blood sample, the force per platelet can then be extrapolated from the time track of contractile forces of the sample. Typical forces per platelet were found to be $\sim 0.4 \mathrm{nN}$ after 15 min clotting time [10. Using a similar device with a flat cup and lid, Jen and McIntire [25] measured forces per platelet of $\sim 0.5 \mathrm{nN}$ in a platelet rich plasma sample after 80 min clotting time (figure I.12 b)). Contrary to the first device, however, Jen and McIntire measured the elastic modulus of the clot with a rheology approach. That is, they applied small amplitude rotational oscillations to the cup instead of using a periodic vertical compressive force on the lid.

Both methods are designed to study platelet contraction by measurements of large cell aggregates. Consequently, platelet forces are accessed indirectly by extrapolation from the total contractile force of the sample. Moreover, the experimental conditions are difficult to control as the blood plasma in the sample contains several clotting agents. Also, in both methods external forces are applied to the sample, which may influence clot formation. In a recent approach, Liang et al. 33] circumvent some of these problems by washing platelets in buffer prior to experiments and by looking at samples with reduced sizes, that is, by studying "microclots". Furthermore, they avoid application of external forces and measure microclot contraction on a dense array of elastic microposts, which serve as force sensors (figure I.12 c)). Contractile forces are inferred from the deflection of the microposts according to the elastic behavior of a cantilever. The magnitude of all forces at the deflected microposts are summed up and divided by the platelet count in the microclot. By these means platelet forces of $2.1 \mathrm{nN}$ were measured after 60 min clotting time. Although Liang et al. reduce clotinfluencing factors in their experiments, the experimental conditions remain difficult to control. One of the main reasons for this, is that platelets themselves release clotting agents upon activation (see section I.1.2, pp. 11). All the while, force extrapolation to single platelets from a total clot force may underestimate the contractile strength of a platelet. For one thing, many forces will balance inside a clot. For another, the links between platelets do not resemble rigid rods, that is, forces are not transmitted 
a)

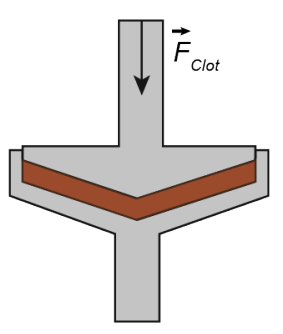

c)

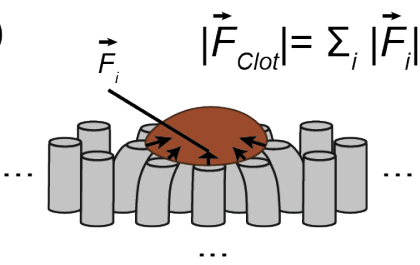

b)

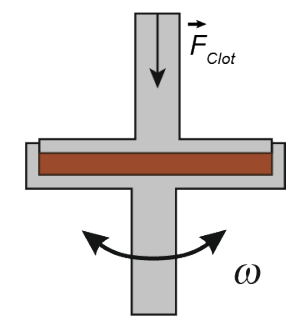

d)

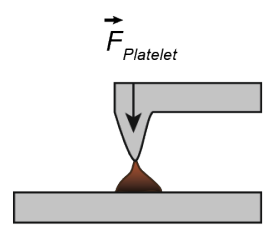

Figure I.12: State-of-the-art measurements of platelet contractility: In the device that was developed by Carr Jr. et al. [8, 10] whole blood samples are placed in a conical cup that is covered by a conical lid (a). As the sample begins to contract during blood clotting, the gap size between cup and lid is reduced. By monitoring the gap size and by periodically calibrating the device with externally applied compressive forces, Carr et al. were able to infer the contractile forces that were generated by the sample. Jen and McIntire [25] used a similar device with a flat cup and lid (b), but opted for an externally driven rotational oscillation of the cup to calibrate the device. In yet a different approach, Liang et al. [33] studied microclot contraction on a dense array of elastic microposts (c). Here, they deduced the force acting on each micropost from the post's deflection and summed up the force magnitude at each post to obtain the overall clot force. Going still to smaller sample sizes, Lam et al. 28. finally analyzed uniaxial contraction of single platelets using AFM. [Figure based on references [8, 25, 33, 28]]

perfectly so that single cell forces very likely add up nonlinearly to the overall clot force. To directly access platelet contractility, single-cell experiments are therefore essential.

Very recently, one such single-cell approach has been realized by Lam et al. [28]. In their study, they have placed single platelets in between a fibrinogen-coated glass surface and a fibrinogen-coated AFM cantilever. By monitoring the deflection of the cantilever they were able to measure contractile forces along the axis perpendicular to the glass surface. Thus, they obtained uniaxial single-cell forces averaging about $29 \mathrm{nN}$ after 15 min clotting time. The fact that these forces are an order of magnitude higher than previously reported underlines the importance of the single-cell approach. Our own work therefore picks up on the single-cell approach, adding two new features to the measurement. On the one hand, we place platelets on soft substrates with an elasticity that is closer to the physiologically relevant range $\left(\sim 4 \mathrm{kP} \mathrm{a}^{[11]}\right)$. On the other hand, we resolve a 2-D force density field for each platelet at several time points during platelet activation. Note that during spreading platelets become very thin both in vitro and in vivo, so that forces generated will mostly lie in a 2-D plane. Our

\footnotetext{
11 For comparison: collagen I, which in vivo plays an important role in platelet adhesion to a site of injury (section I.1.2, has typically an elasticity of about $17 \mathrm{kPa}$ [50].
} 
approach hence measures all relevant forces for platelet contraction. The technique that we use to measure forces both with spatial and temporal resolution is known as traction force microscopy (TFM). TFM is a well-established technique for cells such as fibroblasts [11, 41, 49], fish keratocytes [44] and airway smooth muscle cells [7, 55]. All of these cells are, however, larger than platelets by at least a factor of 10 [41, 44, 55]. We therefore need to adapt the technique to the challengingly small size of platelets $(2-5 \mu \mathrm{m}[38, \mathrm{~s}$. 45]). This adaptation involves aspects that are explained in more detail in the next section, which also contains a review of TFM as an experimental technique.

In addition to measuring platelet forces with TFM, we aim to identify the important mechanisms of force generation and transduction in platelets. In particular, we would like to compare platelets to other contractile cells to conclude upon general principles of cellular contraction (see also section I.2). To achieve this goal, we perform additional and separate experiments, where we fix platelets at different stages during activation on soft substrates and stain cytoskeletal components as well as force transduction sites (focal adhesions). We adopt this indirect imaging approach with fixed instead of living cells, because platelets cannot be easily transfected to fluoresce while living. The reason for this is that platelets lack a DNA-containing nucleus. Here, again, we introduce a novelty by looking at platelet internal rearrangements during activation on soft substrates. Previous studies of platelet actin and myosin were performed on stiff glass cover slips. For instance, Tanaka and Itoh [54] have discovered that after $30 \mathrm{~min}$ of platelet activation on uncoated glass surfaces actin and myosin co-localize. They are both distributed throughout the cell body and the outer regions of the cell, partly forming stress-fiber like bundles. In some cells they also concentrate at the location of granules in the cell center. Furthermore, myosin in the cell body forms granular patches, each granule corresponding to a cluster of myosin filaments. As a peculiarity, Tanaka et al., have noticed that myosin is absent in filopodia.

\section{I.4 Traction Force Microscopy (TFM)}

TFM measures forces per unit area, so called traction forces $\vec{T}(\vec{x})$. To this end, cells are placed on elastic polyacrylamide (PAA) substrates that are coated with an adhesive protein (fibrinogen for platelets) to provide cell anchorage. PAA is specially chosen as a material, because it has a number of convenient properties [59]. On the one hand, it remains linearly elastic for varying degrees of applied strain (see also figure III.3 in section III.3). On the other hand, PAA has elasticities that are in the physiological range $\left(\sim 1-100 \mathrm{kPa} a^{[12]}\right)$. Also, PAA elasticities can be tuned by adjusting the acrylamide monomer to crosslinker (bis-acrylamide) ratio during preparation of the PAA substrates (see also section III.2). Finally, PAA is translucent so that cellular behavior can be observed by microscopy techniques after cells have been seeded on the substrates. All of these characteristics make PAA a most suitable material for TFM experiments on biological samples. The linear elastic behavior of PAA, in particular, is

\footnotetext{
12 according to protocols by Yu-Li Wang (Department of Biomedical Engineering, Carnegie Mellon University, Pittsburgh, PA, USA)
} 
important for TFM, as cellular force generation is measured via cell-induced substrate deformations. Substrate deformations, in turn, are quantified with fluorescent marker beads that are incorporated into the topmost layer of the substrate. As soon as the cells start to deform the substrate, these beads get displaced, which is viewed with fluorescence microscopy. Simultaneously, the cell-substrate contact area is observed by taking phase contrast, DIC or brightfield images of the cells. From the information on the bead displacements $\vec{d}\left(\vec{x}^{\prime}\right)$, the cell-substrate contact area $A$ and the elastic properties of the substrate, the traction forces $\vec{T}(\vec{x})$ can be calculated based on linear elasticity theory by solving the following inversion problem [11]:

$$
d_{j}\left(\vec{x}^{\prime}\right)=\int_{A} G_{i j}\left(\vec{x}^{\prime}-\vec{x}\right) T_{i}(\vec{x}) d x_{1} d x_{2}
$$

where the Green's function $G_{i j}$ is approximated by Boussinesq's solution for semiinfinite, elastic media (see section II.1.2). Usually, in a TFM experiment single time shots are taken from cells after incubation on the substrate overnight. However, it is possible to study the temporal evolution of traction force fields with TFM by simultaneously taking fluorescence images of the substrate and, for example, phase contrast images of the cells for a series of time points. Such time sequences have, in fact, been measured for fibroblasts [41] and airway smooth muscle cells [55] with a spatial resolution of 3-4 $\mu \mathrm{m}$ or $2.7 \mu \mathrm{m}$, respectively, and a temporal resolution of $40 \mathrm{~s}$.

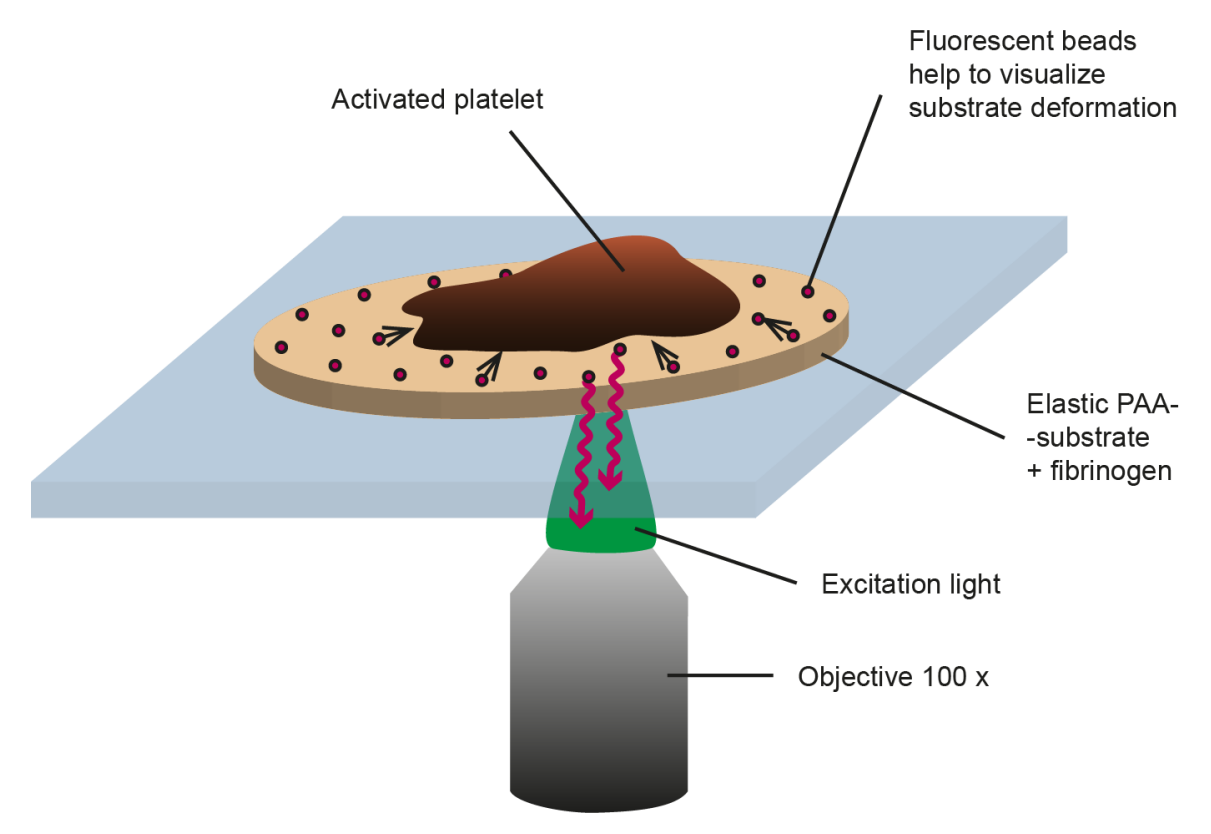

Figure I.13: Sketch of a TFM experimental setup at the example of platelets: Platelets are placed on soft, elastic PAA substrates, where they adhere to the fibrinogencoating at the surface. As soon as the platelets contract, the resulting substrate deformation is visualized with the help of fluorescent marker beads within the substrate. From the bead displacements, spatial maps of cellular forces can then be calculated using linear elasticity theory. 
It should be noted that equation I.1 is an ill-posed problem [6, pp. 183], meaning that a small perturbation in the displacement fields, which is introduced, for example, in the form of noise, leads to large variations in the calculated traction forces. To tackle this problem and also to calculate the bead displacement maps from the TFM images, several approaches have been developed [58].

Butler et al. [7], for example, obtained the bead displacements, or rather the substrate deformation maps, by subdividing the fluorescence images of the undistorted ('null image') and the deformed substrate ('stressed images') into an equal, fixed set of overlapping windows and by cross-correlating each window in the 'null image' in Fourier space with its counterpart in the 'stressed images'. By setting both the substrate deformation maps as well as the traction forces to grid points, they circumvented the ill-conditioning of the inversion problem in equation I.1 and implicitly cut off high frequency contributions to the traction force field (see also [58]). For faster computation, they calculated traction forces in Fourier space and considered both a scenario, where traction forces are distributed unconstrainedly and a scenario, where they are restricted to the cell area. The latter scenario, although more physiological, has a tendency to overestimate traction forces especially at the cell boundary. Additionally, another source of noise is introduced, as the accuracy of calculated traction forces will depend on the accuracy of the estimated cell contour.

While Dembo and Wang [35, 43, 11] also used a correlation technique to arrive at the substrate deformation maps, they calculated the cross-correlations in real space and kept the set of windows for the 'stressed images' flexible. In their implementation, each window in a 'stressed image' was moved within a search box and a final quadratic interpolation was employed to find the optimal match between window pairs. From the substrate deformation, they solved equation I.1 with a regularization, where again forces were calculated in real space on a grid across the cell area. Furthermore, they considered the far-field bead displacements to estimate the probability of a 'null' field (traction forces are zero) ${ }^{[13]}$

Using micropatterned elastomers, instead of PAA with marker beads, Schwarz et al. [52] have also used a regularization to calculate traction forces. They, however, have constrained traction forces to be at discrete focal adhesion points known from GFPvinculin. Here, GFP-tagged vinculin was obtained by transfecting the cells under study.

Yang et al. 63. opted for yet another approach to calculate bead displacement maps, which takes local rotations into account: They focused on the line segments connecting next neighbor beads in the 'null image' and identified matching line segments in the 'stressed image' according to elongation and rotation criteria. Having established matching line segments, the displacement vectors were calculated from the bead positions at the ends of the line segments. For their calculation on the traction forces, Yang et al. used a forward 3-D finite-element-method (FEM) formulation, where they modeled the substrate with fixed boundary conditions imposed on the bottom surface, with prescribed displacements on the top surface according to measured bead displacements and with all other displacements described as a function of the displacements

\footnotetext{
13 according to the manual for the LIBTRC program written by Prof. Micah Dembo (Boston University, MA, USA)
} 
on top and bottom. By choosing this method over solving equation I.1 in the approximation of Boussinesq's solution, they took effects of the finite substrate thickness into account and also provided an estimate for the error introduced by Boussinesq's solution. They find that for increasingly thinner substrates the displacements decay faster than $1 / r$. Boussinesq's solution therefore underestimates traction forces, if substrates get too thin.

In our experiments, we follow the approach by Dembo and Wang, although we have made a few adjustments due to the fact that our cell system, the blood platelets, is considerably smaller than typical cells that are measured with TFM (see also previous section I.3). Thus, in our case, the standard correlation technique that is commonly used to evaluate bead displacements is unable to track the bead patterns during platelet contraction. The main reason for this is that bead patterns change too rapidly under the influence of the large forces that the small platelets exert on their environment. Instead we have programmed our own particle tracking approach to obtain bead displacement fields (see section IV.2). Also, we have recorded long TFM movies to look at the temporal evolution of platelet contraction. As mentioned earlier in this section this approach is rather uncommon for TFM experiments. Usually, in a TFM experiment only three images are taken: one image showing the fluorescent beads in the deformed state of the substrate, another image showing the cells that cause the substrate deformation and finally a reference image of the fluorescent beads in the undeformed substrate after cells have been removed. Hence in the standard procedure, there is only one cell contour that needs to be traced to evaluate a TFM data set. For this reason, cell contours are often manually traced in the community. However, our vast amount of recorded cell images has obliged us to write a program that automatically performs cell contour tracing (see section IV.3). All of these adjustments to TFM data analysis have been further complemented by changes in experimental parameters such as bead size and bead density that we have performed to be able to apply TFM to our small blood platelets (see sections III.2 and III.4 for the final settings). 



\section{Theory}

In the previous section, we have introduced our experimental technique, TFM, and reviewed some of the approaches that are commonly used to evaluate TFM data. Furthermore, we have given an overview of our own approach to TFM data analysis and highlighted adjustments to TFM, which we perform to be able to study force evolution during platelet contraction. Before we describe our own work in more detail, however, we present in this chapter (section II.1) the theoretical background to TFM based on reference [29] (sections 1.2.-1.5., 1.7, 1.8. and 1.10.). In particular, we give an expression for $G_{i j}$ in equation I.1 of the previous section, the so-called Boussinesq solution. This is then followed by a summary of selected state-of-the-art mechanical models to describe cellular contraction (section II.2), which we will later use as a reference to discuss our own results.

\section{II.1 Elastic Deformation of an Isotropic Medium}

\section{II.1.1 Thermodynamics of Elastic Deformation}

For any medium, the internal stress is defined by the following stress tensor

$$
\sigma_{i j}=\frac{F_{i}^{\prime}}{\mathrm{d} A_{j}^{\prime}}
$$

where $F_{i}^{\prime}$ is the force along the $x_{i}$-direction acting on the area $\mathrm{d} A_{j}^{\prime}$ with normal along the $x_{j}$-direction of an infinitesimal cube (see sketch II.1). In the absence of rotations, the tensor is symmetric, that is: $\sigma_{i j}=\sigma_{j i}$. The net force acting, for example, in $x_{2}$-direction on the planes of the cube with normal along $x_{1}$ is given by $\left(\sigma_{21}\left(x_{1}+\mathrm{d} x_{1}, x_{2}, x_{3}\right)-\sigma_{21}\left(x_{1}, x_{2}, x_{3}\right)\right)$.

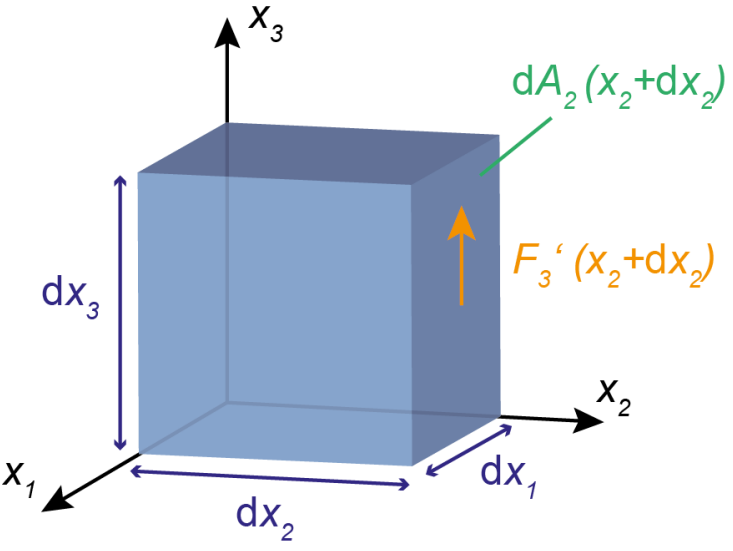

Figure II.1: Sketch of an infinitesimally small volume $\mathrm{d} A_{1}^{\prime}=\partial \sigma_{21}\left(x_{1}, x_{2}, x_{3}\right) / \partial x_{1} \cdot \mathrm{d} V$, where $\mathrm{d} V$ is the volume of the cube $[1]$. The total force is accordingly

$$
F_{i}=\sum_{j} \frac{\partial \sigma_{i j}}{\partial x_{j}} \mathrm{~d} V
$$

\footnotetext{
${ }^{1}$ in the undeformed state
} 
for $i=1,2,3$. If we now assume that the total force for each infinitesimal volume in the medium has effected a small dislocation $\delta \vec{u}(\vec{x})$ of these infinitesimal volumes, then this will lead to the following expression for the work $w$ per unit volume $\mathrm{d} V$ performed by the internal stresses in the medium

$$
\begin{aligned}
\int_{V} w \mathrm{~d} V & =\int_{V} \sum_{i j} \frac{\partial \sigma_{i j}}{\partial x_{j}} \delta u_{i} \mathrm{~d} V \\
& =\int_{V}\left(\sum_{i j} \frac{\partial\left(\sigma_{i j} \cdot \delta u_{i}\right)}{\partial x_{j}}\right)-\left(\sum_{i j} \sigma_{i j} \frac{\partial\left(\delta u_{i}\right)}{\partial x_{j}}\right) \mathrm{d} V
\end{aligned}
$$

According to Gauss' theorem[2] this corresponds to:

$$
\int_{V} w \mathrm{~d} V=\oint_{A} \sum_{i j} \sigma_{i j} \cdot \delta u_{i} \mathrm{~d} A_{j}-\int_{V}\left(\sum_{i j} \sigma_{i j} \frac{\partial\left(\delta u_{i}\right)}{\partial x_{j}}\right) \mathrm{d} V
$$

If we consider a medium with infinite proportions, where the deformation at infinity is zero, then the first term in equation II.4 disappears. Using the symmetry of the stress tensor we can write:

$$
\int_{V} w \mathrm{~d} V=-\frac{1}{2} \int_{V} \sum_{i j} \sigma_{i j}\left(\frac{\partial\left(\delta u_{i}\right)}{\partial x_{j}}+\frac{\partial\left(\delta u_{j}\right)}{\partial x_{i}}\right) \mathrm{d} V
$$

where $\frac{1}{2}\left(\frac{\partial\left(\delta u_{i}\right)}{\partial x_{j}}+\frac{\partial\left(\delta u_{j}\right)}{\partial x_{i}}\right)=$ : $\mathrm{d} \epsilon_{i j}$ for very small displacements $\delta \vec{u}$ corresponds to an infinitesimal part of the strain tensor $\epsilon_{i j}$. If the deformation of the medium occurs sufficiently slowly and is not too large, then the process takes place at thermal equilibrium and it will be reversible $[3]$. The change of internal energy per volume element $u_{I}$ will then be the change in thermal energy $q$ and the work performed by the internal stresses:

$$
\mathrm{d} u_{I}=\mathrm{d} q+\sum_{i j} \sigma_{i j} \mathrm{~d} \epsilon_{i j} \stackrel{\text { rev. }}{=} T \mathrm{~d} s+\sum_{i j} \sigma_{i j} \mathrm{~d} \epsilon_{i j}
$$

where $T$ is the temperature and $s$ is the entropy per volume element. In terms of free energy per volume element $f=u_{I}-T s$ the equation reads:

$$
\mathrm{d} f=s \mathrm{~d} T+\sum_{i j} \sigma_{i j} \mathrm{~d} \epsilon_{i j}
$$

For sufficiently small deformations, we can expand $f$ with respect to the strain tensor. In particular, we expand equation II.7 around the undeformed state of the medium, where the internal stresses $\sigma_{i j}^{0}$ and the strain $\epsilon_{i j}^{0}$ are zero and the temperature $T$ is the

\footnotetext{
2 for any vector field $\vec{P}(\vec{x}): \int_{V} \vec{\nabla} \cdot \vec{P} \mathrm{~d} V=\oint_{A} \vec{P} \cdot \mathrm{d} \vec{A}$

${ }^{3}$ In fact, in the elastic regime of the material, the medium will go back to its original shape as soon as the internal stresses stop to act on the body.
} 
same as in the deformed state:

$$
f \approx f^{0}+\sum_{i j} \underbrace{\left(\frac{\partial f}{\partial \epsilon_{i j}}\right)_{\epsilon_{i j}=0}}_{=\sigma_{i j}^{0}=0} \epsilon_{i j}+\frac{1}{2} \sum_{k l} \sum_{i j} \underbrace{\left(\frac{\partial^{2} f}{\partial \epsilon_{i j} \partial \epsilon_{k l}}\right)_{\epsilon_{i j}=0, \epsilon_{k l}=0}}_{=: C_{i j k l}} \epsilon_{i j} \epsilon_{k l}
$$

In the equation above, $C_{i j k l}$ represents the elastic tensor, which relates the stress tensor to the strain tensor:

$$
\sigma_{i j}=\frac{\partial f}{\partial \epsilon_{i j}}=\sum_{k l} C_{i j k l} \epsilon_{k l}
$$

Due to the symmetry of both the stress and strain tensor and because the partial derivatives in equation II.8 can be interchanged, the elastic tensor can in general be related to 21 unique elastic constants (that is, the following relations are valid: $C_{i j k l}=C_{j i k l}=C_{j i l k}=C_{i j l k}$ and $\left.C_{i j k l}=C_{k l i j}\right]^{[4]}$. In case of isotropic media, that means rotational invariance, this is further reduced to 2 unique constants. For such a medium, equation II.9 can be simplified, if the strain tensor is rewritten to consist of two components, one representing a homogeneous dilation and one representing pure shear [5]

$$
\epsilon_{i j}=\underbrace{\frac{1}{3} \delta_{i j} \sum_{k} \frac{\partial u_{k}}{\partial x_{k}}}_{\text {homogeneous dilation }}+\underbrace{\frac{1}{2}\left(\frac{\partial u_{i}}{\partial x_{j}}+\frac{\partial u_{j}}{\partial x_{i}}\right)-\frac{1}{3} \delta_{i j} \sum_{k} \frac{\partial u_{k}}{\partial x_{k}}}_{\text {pure shear }}
$$

where $\vec{u}(\vec{x})$ is the local deformation. The stress tensor for isotropic media then directly relates to the dilation and shear components of the strain:

$$
\begin{aligned}
\sigma_{i j} & =3 K\left(\frac{1}{3} \delta_{i j} \sum_{k} \frac{\partial u_{k}}{\partial x_{k}}\right)+2 \mu\left(\frac{1}{2}\left(\frac{\partial u_{i}}{\partial x_{j}}+\frac{\partial u_{j}}{\partial x_{i}}\right)-\frac{1}{3} \delta_{i j} \sum_{k} \frac{\partial u_{k}}{\partial x_{k}}\right) \\
& =\lambda \delta_{i j} \sum_{k} \epsilon_{k k}+2 \mu \epsilon_{i j}
\end{aligned}
$$

where $K=\lambda+2 / 3 \mu$ is the bulk modulus, $\lambda$ and $\mu$ are the Lamé parameters. Incidentally, $\mu$ is also the shear modulus of the medium. The linear relationship we have thus established between stress and strain tensor (equation II.11) is effectively Hooke's law for isotropic, elastic media.

Alternatively to writing the relation between stress and strain in terms of the Lamé parameters, it is useful to express it in terms of the elastic modulus or Young's modulus $E$ and the Poisson ratio $\nu$. Both $E$ and $\nu$ relate to a homogeneous deformation of an isotropic medium, where the strain $\epsilon_{i j}$ and the stress $\sigma_{i j}$ are constant throughout the medium. For instance, if we consider the medium to dilate homogeneously in $x_{3}$-direction due to an external stress $\sigma_{33}$, then the Young's modulus relates the strain

\footnotetext{
4 The tensor is often also written as a $6 \times 6$ tensor, $C_{n m}$, where the values $1,2,3,4,5,6$ for $\mathrm{n}$ or $\mathrm{m}$ correspond to ij- or kl-pairs of $11,22,33,23,13,12$.

5 'Pure shear' refers to shear without volumetric changes, meaning that the sum of the diagonal elements of this component is zero.
} 
$\epsilon_{33}$ to $\sigma_{33}$ :

$$
\sigma_{33}=E \epsilon_{33}
$$

In our example, the Poisson ratio would then describe the contraction in the $x_{2}$ - $x_{1}$-plane caused by the dilation in $x_{3}$-direction:

$$
\epsilon_{22}=\epsilon_{11}=-\nu \epsilon_{33}
$$

where $\nu$ has values between 0 and 0.5 depending on the compressibility of the medium. Considering that all components of $\sigma_{i j}$ except $\sigma_{33}$ are zero in our example, we can set e.g. $\sigma_{11}=0$ in equation II.11. Furthermore, by inserting equations II.12 and II.13 into II.11 for $\sigma_{33}$ and $\sigma_{11}(=0)$, we obtain the following general relationships:

$$
\mu=\frac{E}{2(1+\nu)} ; \lambda=\frac{E \nu}{(1-2 \nu)(1+\nu)} ; K=\frac{E}{3(1-2 \nu)}
$$

With equations II.14 and II.11, we arrive at the final expression:

$$
\sigma_{i j}=\frac{E}{1+\nu}\left(\epsilon_{i j}+\frac{\nu}{1-2 \nu} \delta_{i j} \sum_{k} \epsilon_{k k}\right)
$$

\section{II.1.2 Deformation of a Semi-Infinite Elastic Medium}

For a semi-infinite, isotropic and elastic medium in equilibrium the local internal forces per volume element should be zero:

$$
\frac{F_{i}}{\mathrm{~d} V} \stackrel{\text { eq. }}{=} \sum_{j} \frac{\partial \sigma_{i j}}{\partial x_{j}} \text { eq. } \amalg .15 . \Pi .10 \frac{2 E}{1+\nu}\left(\left(\vec{\nabla}^{2}\right) \vec{u}+\frac{1}{(1-2 \nu)} \vec{\nabla}(\vec{\nabla} \vec{u})\right) \stackrel{!}{=} 0
$$

The equation that needs to be solved for the local deformation $\vec{u}(\vec{x})$ is therefore:

$$
\vec{\nabla}(\vec{\nabla} \vec{u})+(1-2 \nu)\left(\vec{\nabla}^{2}\right) \vec{u}=0
$$

Equation II.17 can be solved analytically in cases, where the deformation and the forces generating the deformation disappear for $|\vec{x}| \rightarrow \infty$. We consider the following Ansatz:

$$
\vec{u}(\vec{x})=\vec{h}(\vec{x})+\vec{\nabla} \varphi(\vec{x}) \quad \text { with } \quad\left(\vec{\nabla}^{2}\right) \vec{h}(\vec{x})=0
$$

If we insert our Ansatz into equation II.17 and take advantage of the fact that $\left(\vec{\nabla}^{2}\right) \vec{\nabla} \varphi=$ $\vec{\nabla}\left(\left(\vec{\nabla}^{2}\right) \varphi\right)$, we obtain the following condition:

$$
\vec{\nabla} \underbrace{\left[(\vec{\nabla} \vec{h})+2(1-\nu)\left(\left(\vec{\nabla}^{2}\right) \vec{\varphi}\right)\right]}_{=: L(\vec{x})}=0
$$


Equation II.19 actually represents three equations for the scalar function $L(\vec{x})$, which are satisfied if $L$ is a constant $c_{L}$ :

$$
\frac{\partial L(\vec{x})}{\partial x_{1}}=0 ; \frac{\partial L(\vec{x})}{\partial x_{2}}=0 ; \frac{\partial L(\vec{x})}{\partial x_{3}}=0 \Rightarrow L(\vec{x})=c_{L}
$$

We consider $\vec{h}$ and $\varphi$ to be such that $c_{L}=0$. Equation II.19 can then be reformulated:

$$
2(1-\nu)\left(\left(\vec{\nabla}^{2}\right) \vec{\varphi}\right)=-(\vec{\nabla} \vec{h})
$$

If we set the surface of the semi-infinite, elastic medium to the $x_{1}-x_{2}$-plane, it is convenient to write $h_{1}$ and $h_{2}$ as derivatives of some functions $g_{1}$ and $g_{2}$ :

$$
h_{1}=\frac{\partial g_{1}}{\partial x_{3}} ; \quad h_{2}=\frac{\partial g_{2}}{\partial x_{3}}
$$

Note that $g_{1}$ and $g_{2}$ are not fully defined by equation II.22. In fact, any $g_{1}^{\prime}=$ $g_{1}+p_{1}\left(x_{1}, x_{2}\right)$ and $g_{2}^{\prime}=g_{2}+p_{2}\left(x_{1}, x_{2}\right)$ with arbitrary functions $p_{1}$ and $p_{2}$ will satisfy equation II.22 as well. We can therefore choose $g_{1}$ and $g_{2}$ such that $\left(\vec{\nabla}^{2}\right) g_{1}=0$ and $\left(\vec{\nabla}^{2}\right) g_{2}=0$. Equation II.21 then has the following appearance:

$$
2(1-\nu)\left(\left(\vec{\nabla}^{2}\right) \vec{\varphi}\right)=-\frac{\partial}{\partial x_{3}} \underbrace{\left(\frac{\partial g_{1}}{\partial x_{1}}+\frac{\partial g_{2}}{\partial x_{2}}+h_{3}\right)}_{=: M(\vec{x})}
$$

We chose $\left(\vec{\nabla}^{2}\right) h_{3}=0,\left(\vec{\nabla}^{2}\right) g_{1}=0$ and $\left(\vec{\nabla}^{2}\right) g_{2}=0$ in our Ansatz, hence $\left(\vec{\nabla}^{2}\right) M=0$. We can thus rewrite equation II.23.

$$
2(1-\nu)\left(\left(\vec{\nabla}^{2}\right) \vec{\varphi}\right)=-\frac{\partial M}{\partial x_{3}}=-\frac{\partial M}{\partial x_{3}}-\frac{1}{2} x_{3} \underbrace{\left(\vec{\nabla}^{2}\right) M}_{=0}=\left(\vec{\nabla}^{2}\right)\left(-\frac{1}{2} x_{3} M\right)
$$

The solution to $\varphi$ is then:

$$
\varphi(\vec{x})=-\frac{x_{3}}{4(1-\nu)}\left(\frac{\partial g_{1}}{\partial x_{1}}+\frac{\partial g_{2}}{\partial x_{2}}+h_{3}\right)+\psi
$$

where $\psi$ is yet another function with $\left(\vec{\nabla}^{2}\right) \psi=0$.

Additionally, the functions $g_{1}, g_{2}, h_{3}$ and $\psi$ have to satisfy the boundary condition $\sigma_{i 3}=-P_{i}$, where $P_{i}$ represents the externally applied stress at the surface of the elastic medium. Combining equation II.15, II.10, II.18, II.22 and II.25 the boundary conditions yield:

$$
\begin{aligned}
& \left.\frac{\partial^{2} g_{1}}{\partial x_{3}^{2}}\right|_{x_{3}=0}+\left.\frac{\partial}{\partial x_{1}}\left\{\frac{1-2 \nu}{2(1-\nu)} h_{3}-\frac{1}{2(1-\nu)}\left(\frac{\partial g_{1}}{\partial x_{1}}+\frac{\partial g_{2}}{\partial x_{2}}\right)+2 \frac{\partial \psi}{\partial x_{3}}\right\}\right|_{x_{3}=0} \\
& =-\frac{2(1+\nu)}{E} P_{1}
\end{aligned}
$$




$$
\begin{array}{r}
\left.\frac{\partial^{2} g_{2}}{\partial x_{3}^{2}}\right|_{x_{3}=0}+\left.\frac{\partial}{\partial x_{2}}\left\{\frac{1-2 \nu}{2(1-\nu)} h_{3}-\frac{1}{2(1-\nu)}\left(\frac{\partial g_{1}}{\partial x_{1}}+\frac{\partial g_{2}}{\partial x_{2}}\right)+2 \frac{\partial \psi}{\partial x_{3}}\right\}\right|_{x_{3}=0} \\
=-\frac{2(1+\nu)}{E} P_{2} \\
\left.\frac{\partial}{\partial x_{3}}\left\{h_{3}-\left(\frac{\partial g_{1}}{\partial x_{1}}+\frac{\partial g_{2}}{\partial x_{2}}\right)+2 \frac{\partial \psi}{\partial x_{3}}\right\}\right|_{x_{3}=0}=-\frac{2(1+\nu)}{E} P_{3}
\end{array}
$$

However, the four functions $g_{1}, g_{2}, h_{3}$ and $\psi$ are not fully defined by the three equations II.26 II.28, We can therefore choose a set $g_{1}, g_{2}, h_{3}$ and $\psi$, which satisfies additional, convenient conditions. For example we can choose a set, where the curly brackets in equations II.26 and II.27 are zero:

$$
(1-2 \nu) h_{3}-\left(\frac{\partial g_{1}}{\partial x_{1}}+\frac{\partial g_{2}}{\partial x_{2}}\right)+4(1-\nu) \frac{\partial \psi}{\partial x_{3}}=0
$$

Equation II.26 and II.27 then simplify to:

$$
\left.\frac{\partial^{2} g_{1}}{\partial x_{3}^{2}}\right|_{x_{3}=0}=-\frac{2(1+\nu)}{E} P_{1} ;\left.\quad \frac{\partial^{2} g_{2}}{\partial x_{3}^{2}}\right|_{x_{3}=0}=-\frac{2(1+\nu)}{E} P_{2}
$$

With equations II.28 II.30 we can now calculate $g_{1}, g_{2}, h_{3}$ and $\psi$ exactly.

Before we consider any generalized external stress or traction force field $T_{i}(\vec{x})$, however, we look at the special case of a point force which gives an external stress of $\vec{P}=\vec{F} \delta x_{1} \delta x_{2}$ acting on the elastic medium at the origin of our coordinate system. According to potential theory, any potential $\phi$ that disappears at $|\vec{x}| \rightarrow \infty$ and has its normal derivative in the plane $x_{3}=0$ can be expressed in terms of:

$$
\phi(\vec{x})=-\left.\frac{1}{2 \pi} \int \frac{\partial \phi\left(x_{1}^{\prime}, x_{2}^{\prime}, x_{3}\right)}{\partial x_{3}}\right|_{x_{3}=0} \frac{\mathrm{d} x_{1}^{\prime} \mathrm{d} x_{2}^{\prime}}{\left|\vec{x}-\vec{x}^{\prime}\right|}
$$

with $\vec{x}^{\prime}=\left(x_{1}^{\prime}, x_{2}^{\prime}, 0\right)$. If we apply this to equations II.28 and II.30, we obtain with $r:=|\vec{x}|:$

$$
\begin{aligned}
& h_{3}-\left(\frac{\partial g_{1}}{\partial x_{1}}+\frac{\partial g_{2}}{\partial x_{2}}\right)+2 \frac{\partial \psi}{\partial x_{3}}=\frac{1+\nu}{\pi E} \int \frac{P_{3}\left(x_{1}^{\prime}, x_{2}^{\prime}\right)}{\left|\vec{x}-\vec{x}^{\prime}\right|} \mathrm{d} x^{\prime} \mathrm{d} y^{\prime}=\frac{1+\nu}{\pi E} \frac{F_{3}}{r} \\
& \frac{\partial g_{1}}{\partial x_{3}}=\frac{1+\nu}{\pi E} \frac{F_{1}}{r} ; \quad \frac{\partial g_{2}}{\partial x_{3}}=\frac{1+\nu}{\pi E} \frac{F_{2}}{r}
\end{aligned}
$$

As $\varphi$ depends on $\partial g_{1} / \partial x_{1}$ and $\partial g_{2} / \partial x_{2}$, we need to find an expression for these two terms to arrive at the final expression of $\vec{u}$. We therefore calculate the derivative of equation II.33 with respect to $x_{1}$ and $x_{2}$ and integrate over $x_{3}^{\prime}$ from $-\infty$ to $x_{3}$. Note 
that, in our case, integrals and derivatives can be exchanged.

$$
\begin{aligned}
\frac{\partial g_{1}}{\partial x_{1}} & =\int_{-\infty}^{x_{3}} \frac{\partial^{2} g_{1}\left(x_{1}, x_{2}, x_{3}^{\prime}\right)}{\partial x_{1} \partial x_{3}^{\prime}} \mathrm{d} x_{3}^{\prime}=-\int_{-\infty}^{x_{3}} \frac{1+\nu}{\pi E} \frac{F_{1} x_{1}}{{\sqrt{x_{1}^{2}+x_{2}^{2}+x_{3}^{\prime 2}}}^{3}} \mathrm{~d} x_{3}^{\prime} \\
& =\frac{1+\nu}{\pi E} \frac{F_{1} x_{1}}{r\left(r+x_{3}\right)} \\
\frac{\partial g_{2}}{\partial x_{2}} & =\int_{-\infty}^{x_{3}} \frac{\partial^{2} g_{2}\left(x_{1}, x_{2}, x_{3}^{\prime}\right)}{\partial x_{2} \partial x_{3}^{\prime}} \mathrm{d} x_{3}^{\prime}=-\int_{-\infty}^{x_{3}} \frac{1+\nu}{\pi E} \frac{F_{2} x_{2}}{{\sqrt{x_{1}^{2}+x_{2}^{2}+x_{3}^{\prime 2}}}^{3}} \mathrm{~d} x_{3}^{\prime} \\
& =\frac{1+\nu}{\pi E} \frac{F_{2} x_{2}}{r\left(r+x_{3}\right)}
\end{aligned}
$$

With equations II.29, II.32, II.34 and II.35 we can then determine $h_{3}$ and $\partial \psi / \partial x_{3}$. From $\partial \psi / \partial x_{3}$ we can determine $\partial \psi / \partial x_{1}$ and $\partial \psi / \partial x_{2}$ by integrating over $x_{3}$ and calculating the derivative for $x_{1}$ and $x_{2}$. These expressions together with equations II.33, II.34 and II.35 can finally be combined with equations II.18, II.22 and II.25 to obtain the final result:

$$
u_{i}=\sum_{k} G_{i k}\left(x_{1}, x_{2}, x_{3}\right) F_{k}
$$

where $G_{i k}$ is given by:

$$
G_{i k}=\beta\left(\begin{array}{lcc}
\frac{2(1-\nu) r+x_{3}}{r\left(r+x_{3}\right)}+\frac{x_{1}^{2}\left(2 r\left(\nu r+x_{3}\right)+x_{3}^{2}\right)}{r^{3}\left(r+x_{3}\right)^{2}} & \frac{x_{1} x_{2}\left(2 r\left(\nu r+x_{3}\right)+x_{3}^{2}\right)}{r^{3}\left(r+x_{3}\right)^{2}} & \frac{x_{1} x_{3}}{r^{3}}-\frac{(1-2 \nu) x_{1}}{r\left(r+x_{3}\right)} \\
\frac{x_{1} x_{2}\left(2 r\left(\nu r+x_{3}\right)+x_{3}^{2}\right)}{r^{3}\left(r+x_{3}\right)^{2}} & \frac{2(1-\nu) r+x_{3}}{r\left(r+x_{3}\right)}+\frac{x_{2}^{2}\left(2 r\left(\nu r+x_{3}\right)+x_{3}^{2}\right)}{r^{3}\left(r+x_{3}\right)^{2}} & \frac{x_{2} x_{3}}{r^{3}}-\frac{(1-2 \nu) x_{2}}{r\left(r+x_{3}\right)} \\
x_{1}\left(\frac{1-2 \nu}{r\left(r+x_{3}\right)}+\frac{x_{3}}{r^{3}}\right) & x_{2}\left(\frac{1-2 \nu}{r\left(r+x_{3}\right)}+\frac{x_{3}}{r^{3}}\right) & \frac{2(1-\nu)}{r}+\frac{x_{3}^{2}}{r^{3}}
\end{array}\right)
$$

the factor $\beta$ being: $\beta:=\frac{1+\nu}{2 \pi E}$.

In mathematical terminology $G_{i k}$ is Green's tensor - the solution for a point force. The solution for any generalized external stress or traction force field $T_{i}\left(x_{1}, x_{2}\right)$, is hence obtained by the integral:

$$
u_{i}=\int \sum_{k} G_{i k}\left(x_{1}-x_{1}^{\prime}, x_{2}-x_{2}^{\prime}, x_{3}\right) T_{k}\left(x_{1}^{\prime}, x_{2}^{\prime}\right) \mathrm{d} x_{1}^{\prime} \mathrm{d} x_{2}^{\prime}
$$

It is often of interest to look at the deformation at the surface of the elastic medium. The expression for $G_{i k}$ (equation II.37) is then significantly reduced by setting $x_{3}=0$ :

$$
G_{i k}\left(x_{1}, x_{2}, x_{3}=0\right)=\frac{1+\nu}{2 \pi E r}\left(\begin{array}{lcc}
2(1-\nu)+\frac{2 \nu x_{1}^{2}}{r^{2}} & \frac{2 \nu x_{1} x_{2}}{r^{2}} & -\frac{(1-2 \nu) x_{1}}{r} \\
\frac{2 \nu x_{1} x_{2}}{r^{2}} & 2(1-\nu)+\frac{2 \nu x_{2}^{2}}{r^{2}} & -\frac{(1-2 \nu) x_{2}}{r} \\
\frac{(1-2 \nu) x_{1}}{r} & \frac{(1-2 \nu) x_{2}}{r} & 2(1-\nu)
\end{array}\right)
$$


Note that for an incompressible material the Poisson ratio $\nu$ is 0.5 and the components $G_{13}, G_{23}, G_{31}$ and $G_{32}$ in equation II.39 are zero. Hence, the components $u_{1}$ and $u_{2}$ of the deformation at the surface depend only on $T_{1}$ and $T_{2}$ (see equation II.38). Likewise, the component $u_{3}$ depends only on $T_{3}$. Consequently, the in-plane and out-ofplane deformation at the surface of an incompressible or near-incompressible material effectively decouple. By monitoring the in-plane deformation, we can therefore access the in-plane traction forces.

\section{II.2 Mechanical Principles of Cellular Contraction}

Several mechanical models have been developed over the years to describe single cell adhesion or the adhesion of entire cell tissue layers to a substrate [22, 4, 65, 12, 30]. These models address aspects of measured cell and tissue shapes, of force distributions across the cell-substrate contact area and of the dynamic reorganization of cellular actin into distinct stress fibers based on given adhesion geometries, initial cell shapes, cell-specific spreading behavior or given substrate elasticities. In the following, a brief summary will be given of a few models, which have been selected for their most promising applicability to our experiments on platelets.

\section{II.2.1 Cell Shape and Force Distribution According to Mechanical Network Models}

A variety of numerical models considering mechanical two-dimensional networks are presented and discussed by Guthardt Torres et al. [22]. All of these computer models assume that adherent cells are flat enough, so that only the two-dimensional cellsubstrate contact area needs to be considered. These network models concentrate on cellular actin and its associated proteins as the prominent mechanical components for cellular adhesion. A hypothetical actin network is represented in the models as an array of mechanical links with a number $N$ of discrete points $\vec{R}_{i}$, the nodes of the network, where the mechanical links are connected (see figure II.3). At mechanical equilibrium, the forces exerted by each link $\vec{F}_{i j}$ on a given node $i$ should add up to zero:

$$
\vec{F}_{i}=\sum_{j} \vec{F}_{i j}=0
$$

The adhesion geometry is then implemented as boundary conditions, where the nodes at the adhesion sites are set to fixed positions. Three types of models are discussed in particular: passive Hookean spring networks (HSN), passive cable networks (PCN) and actively contracting cable networks (ACN). As pointed out by Guthardt Torres et al., any actively contracting Hookean spring network can be represented by passive Hookean springs with a reduced resting length of the individual springs, due to the linear character of Hookean springs. For this reason, the particular network model of actively contracting Hookean springs is not separately considered. 


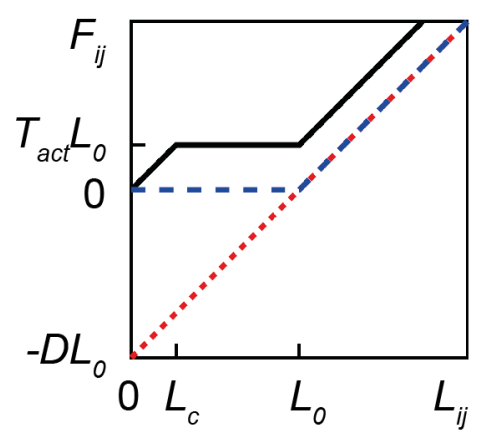

Figure II.2: Force extension curves for the different mechanical network models describing adherent cells: Hookean spring networks (HSN) are represented by a red, dotted line; passive cable networks (PCN) are shown as a blue, dashed line and active cable networks $(\mathrm{ACN})$ are represented by a black, solid line $\left(F_{i j}\right.$ : force acting on node $i$ in direction of node $j, T_{a c t}$ : force per link length in ACNs, $L_{i j}$ : mechanical link length, $L_{0}$ : initial/tensionfree link length, $L_{c}$ : critical link length in ACNs (see equation II.43, $D$ : spring constant). [Figure from [22], labels were adapted]

In general, the HSN is a good, simplified model in the limit of small network deformations. Here, the mechanical links are linear springs that generate restoring forces

$$
\vec{F}_{i j}{ }^{\text {eq. II.12 }} E A \underbrace{\frac{\left(L_{i j}-L_{0}\right)}{L_{0}}}_{\text {strain in spring }} \underbrace{\frac{\vec{R}_{j}-\vec{R}_{i}}{L_{i j}}}_{=: \vec{e}_{i j}}=\underbrace{\frac{E A}{L_{0}}}_{=: D}\left(L_{i j}-L_{0}\right) \vec{e}_{i j}
$$

where $E$ is the Young's modulus of the link $[6], A$ is its cross sectional area ${ }^{[6]}, L_{i j}$ is the length of the link, $L_{0}$ is its resting length ${ }^{[6]}, \vec{R}_{j}$ and $\vec{R}_{i}$ are, as before, node positions in the network, $\vec{e}_{i j}$ is the unit vector along a link and $D$ is the spring constant of the links (see force extension curve in figure II.2). Although the HSN is an appealing, simple model it does not, capture a special mechanical property of actin: its almost nonexistent resistance to compression. Indeed, actin can be stretched, but if compressed it tends to buckle easily, single filaments in an actin bundle slide across each other and at sufficiently high loads actin even depolymerizes. The next best model for actin networks that encompasses its special mechanical properties under load, is therefore a passive cable network. Here, the restoring force of the mechanical cable links (see force extension curve in figure II.2 is given by

$$
\vec{F}_{i j}= \begin{cases}E A \frac{\left(L_{i j}-L_{0}\right)}{L_{0}} \vec{e}_{i j}, & L_{i j}>L_{0} \\ 0, & L_{i j} \leq L_{0}\end{cases}
$$

However different the HSNs and PCNs are in principle, they share many common features, particularly if all mechanical links in the network are tensed. Thus, for both networks there is a reference shape in the absence of adhesion constraints, in which all mechanical links are at their resting length. In the presence of adhesions both HSNs

\footnotetext{
${ }^{6}$ In the article by Guthardt Torres et al. the following values were used: $E A=50 \mathrm{nN}$ and $L_{0}=100 \mathrm{~nm}$.
} 


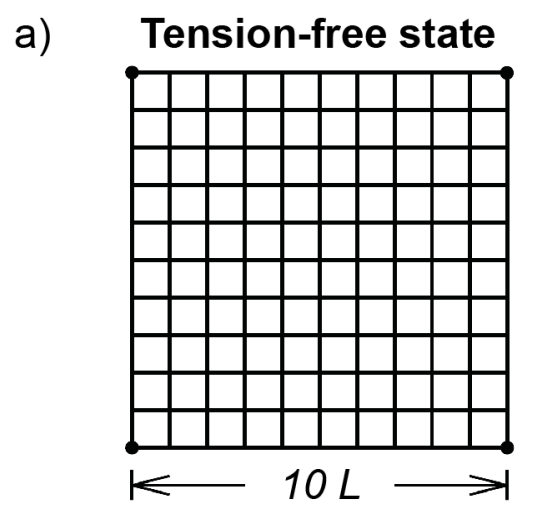

HSN/ PCN

b)

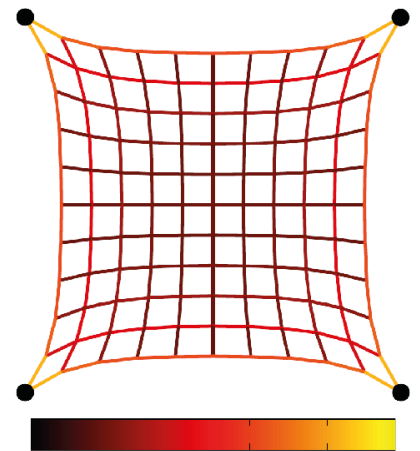

$\begin{array}{lllll}0 & 0.1 & 0.2 & 0.3\end{array}$

F/EA

d)

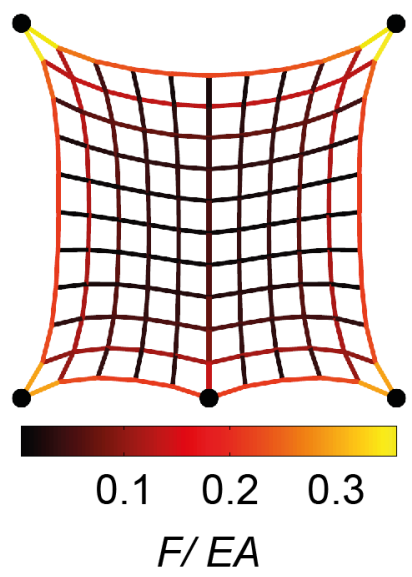

ACN

c)

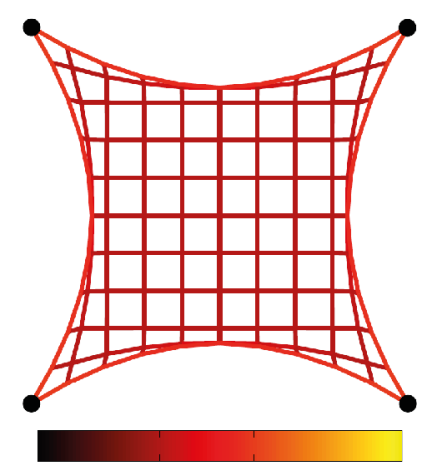

$\begin{array}{llll}10^{-3} & 0.01 & 0.1 & 1\end{array}$

$F / E A$

e)

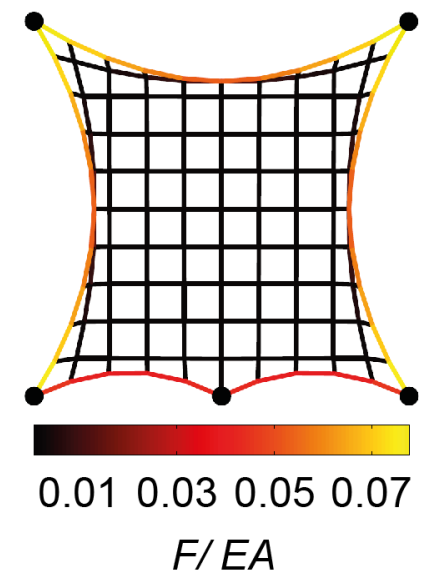

Figure II.3: Network models for adherent cells: a) Initial tension-free state of networks with square lattice topology, in which the linker length $L$ is equal to the resting length $L_{0}$, b)-e) Deformation and color-coded force distribution $F$, given in units of the elastic scaling factor $E A$ : for an HSN under internal tension with $\left(L-L_{0}\right) / L=0.2((\mathrm{~b})$; similar results are obtained for PCNs), for an ACN under tension with force per length $T_{\text {act }}$ given by $T_{a c t} L_{0} / E A=10^{-2}$ (c), for the same HSN/PCN (d) and ACN (e) upon addition of another adhesion site. Note the different scalings of the force distributions. [Figures from [22], labels were adapted] 
and PCNs take on similar shapes and have similar force distributions, where network deformations and hence forces are highest in a region around the adhesion sites and the bulk network approaches the reference state (see figure II.3 b)). Also, both network models show global rather than local responses to adhesion constraints. That is, upon addition, for example, of a new adhesion site, the displacement of mechanical links changes across large regions in the network (see figure II.3 d)). When comparing the shape of HSNs and PCNs for a given adhesion geometry to experimentally observed shapes of adherent cells one finds that the models show strong invaginations, but cannot explain the circular forms observed for invaginations in experiments. The circular arcs are, in fact, explained if the PCN is extended to include active contractility (ACN), which simulates the activity of myosin motors in conjuncture with actin. Under the assumption of a homogeneous myosin distribution throughout the network the restoring forces of the mechanical links in such active cable networks is modeled as follows

$$
\vec{F}_{i j}= \begin{cases}\left(T_{a c t} L_{0}+E A \frac{\left(L_{i j}-L_{0}\right)}{L_{0}}\right) \vec{e}_{i j}, & L_{i j}>L_{0} \\ T_{a c t} L_{0} \vec{e}_{i j}, & L_{c} \leq L_{i j} \leq L_{0} \\ T_{a c t} L_{0} \frac{L_{i j}}{L_{c}} \vec{e}_{i j}, & L_{i j}<L_{c}\end{cases}
$$

where $T_{a c t}$ is the force density per length[[7], $T_{a c t} L_{i j}$ is assumed to be constant (i.e. the total number of motors per mechanical link remains constant during contraction) and the last line in equation II.43 is introduced for a critical length $L_{c}$ to avoid the unphysical case of $\vec{F}_{i j} \neq 0$ for $L_{i j} \rightarrow \mathbb{C}^{[8]}$ (see force extension curve in figure II.2. Although ACNs have no reference state, meaning that they would unphysiologically contract to a single point in the absence of adhesions, they capture the cell shape well for a given adhesion geometry. Furthermore, ACNs respond locally to adhesion constraints so that if a new adhesion point is introduced the network deformation and hence also the force distribution changes only in a small region about the new adhesion point (see figure II.3 e)). For the same reason, the internal force distribution in ACNs is constant in the bulk and strongly localized to the edge of the network. Here, forces are rather high differing by orders of magnitude as compared to the forces in the bulk (see figure II.3 c) and e)). Also, for ACNs the network deformation and force distribution is found to vary little with both varying network topologies and varying adhesion geometries if the size of the adhesion geometry is kept the same. Interestingly, the mechanical links in ACNs bundle along the edge of the network if the network is sufficiently stretched by the imposed adhesion geometry.

\section{II.2.2 Modeling Stress Fiber Polarization}

Although the network models presented in the previous section are useful to explain aspects of cell shape and force distributions for given actin network topologies in adherent cells, they do not elucidate how a given actin network topology comes into

\footnotetext{
7 In the article by Guthardt Torres et al. $T_{\text {act }}$ was set to $2 \cdot 10^{-2} \mathrm{nN} / \mathrm{nm}$.

8 According to Guthardt Torres et al. neither the particular choice of $L_{c}$ nor the fact that the force reduction in the last line of equation II.43 was chosen to be linear, have a significant influence on the results obtained.
} 
existence. To address this question Zemel et al. [65] have introduced another model altogether, in which the cell is modeled in 2-D or 3-D as an ellipsoidal, actively contracting elastic inclusion in the likewise elastic substrate/matrix. Both cell and matrix are assumed to be isotropic, homogeneous and the matrix is additionally taken to be infinite. Furthermore, the cell contributes a passive, elastic response to spreading in addition to the active contractility. While the active contractile forces are attributed to myosin motor activity, the passive, elastic forces are associated with the general elastic properties of the cell, including the elasticity of the plasma membrane in conjunction with the various components of the cytoskeleton.

To calculate the effect of cell spreading on the internal stresses both within the cell and the matrix, Zemel et al. first represent the 3-D case and begin by considering a hypothetical situation (see figure II.4). In this hypothetical situation, they imagine the cell to be stretched to its maximum size, that is, the size it would assume after spreading on/in an infinitely rigid matrix. This particular state of the cell is chosen to be the reference state with associated strain $-\epsilon_{i j, c}^{0}[9]$. The index $c$ is used in this context to indicate that $-\epsilon_{i j, c}^{0}$ relates to the cell. In the following an index $m$ will be used analogously to indicate parameters that relate to the matrix. After maximally stretching the cell, it is brought into contact with the matrix and the forces are allowed to relax in both the cell and the matrix, which leads to the development of a strain $\epsilon_{i j, c}^{r}$ within the cell. The relaxed state finally corresponds to the experimentally observed situation of spread cells on/in a flexible matrix. According to equation II.9 the elastic stress $\sigma_{i j, c}^{r}$ in the final state of the cell can be expressed in terms of the final strain $\epsilon_{k l, c}^{r}-\epsilon_{k l, c}^{0}$ via the elastic tensor $C_{i j k l, c}$ of the cell:

$$
\sigma_{i j, c}^{r}=\sum_{k l} C_{i j k l, c} \underbrace{\left(\epsilon_{k l, c}^{r}-\epsilon_{k l, c}^{0}\right)}_{=: \Delta \epsilon_{k l, c}}[10]
$$

This elastic stress is presumed to initiate the development of stress fibers and influence their orientation within the cell. The stress fibers, in turn, contract actively. The active elements within the cell that cause this contraction are modeled as small force dipoles. Although the force dipoles relate to the myosin motor proteins in the cell, they are not a one-to-one representation of each motor. Furthermore, elastic contributions from acto-myosin are included in the forces of the dipole. Each dipole has an associated dipole moment $\vec{F} \cdot \vec{d}$, where $\vec{F}$ is the force at one end of the dipole and $\vec{d}$ is the distance between the two ends of the dipole. Expressed in terms of a tensor rather than a scalar, the dipoles in the cell can be represented by $p_{i j}(\vec{x})=F_{i}(\vec{x}) d_{j}(\vec{x}) / \mathrm{d} V V^{[11]}$, where $\mathrm{d} V$ is the small volume fraction within the cell that a single dipole occupies. The change in the average dipole per unit volume $\left\langle p_{i j}\right\rangle_{V}$ that is induced by the elastic stress $\sigma_{k l, c}^{r}$ within the cell can then be formulated analogously to the electrostatic case of induced

\footnotetext{
${ }^{9} \epsilon_{i j, c}^{0}<0$ in this notation relates to the transformation that the cell undergoes, as it returns to its elastically undeformed shape.

10 As the cell is assumed to be isotropic and homogeneous, the $C_{i j k l}$ reduce to two constants as before (see section II.1.1). However, we will not specify $C_{i j k l}$ in this chapter to keep expressions short.

${ }^{11} p_{i j}$ corresponds to a force per unit area
} 


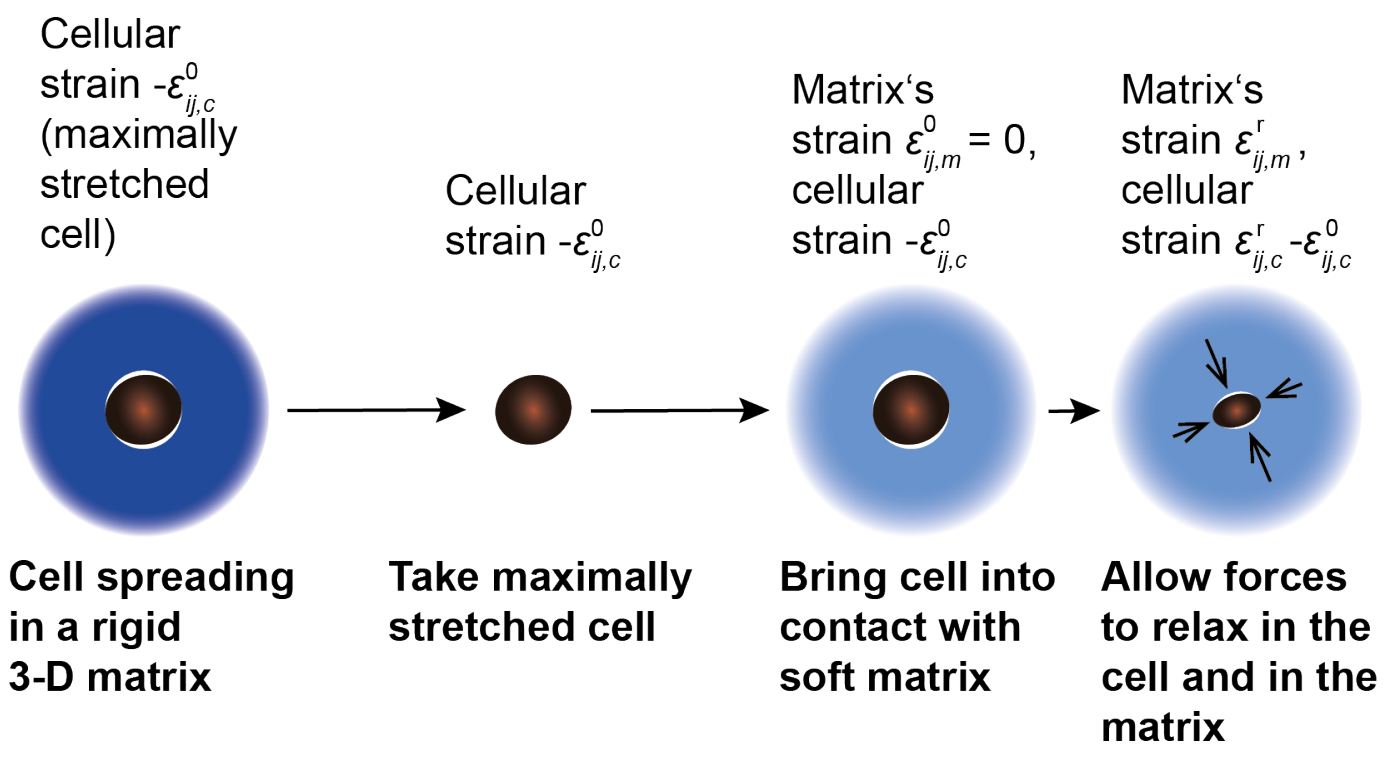

Figure II.4: Sketch illustrating a hypothetical process to deduce the equations describing cell spreading (here in 3-D): The cell is initially stretched to the shape it would assume in an infinitely rigid matrix. During stretching, the cell experiences a strain $-\epsilon_{i j, c}^{0}$. It is then brought into contact with a soft matrix that is initially undeformed. This particular situation is taken to be the reference for future considerations. The forces both in the cell and in the matrix are allowed to relax, so that the cell experiences an additional strain $\epsilon_{i j, c}^{r}$. In the final relaxed state, the cell is under a total strain $\epsilon_{i j, c}^{r}-\epsilon_{i j, c}^{0}$, whereas the matrix is under a strain $\epsilon_{i j, m}^{r}$.

polarity of a charge density in an external electric field:

$$
\begin{aligned}
& \Delta\left\langle p_{i j}\right\rangle_{V}:=\left\langle p_{i j}\right\rangle_{V}-\left\langle p_{i j}^{0}\right\rangle_{V}
\end{aligned}
$$

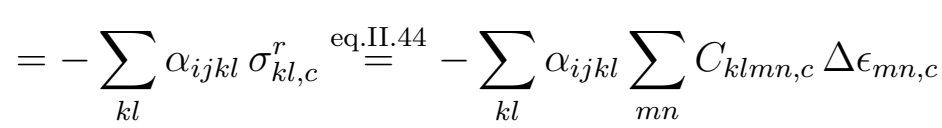

where $\left\langle p_{i j}^{0}\right\rangle_{V}$ is the average dipole per unit volume in the reference state (maximally stretched cell) and $\alpha_{i j k l}$ denotes the cell polarizability tensor. As the stress fiber formation begins when the cytoskeleton is still in an isotropic, homogeneous state $\alpha_{i j k l}$ is approximated to be isotropic, as well. Analogously to equation II.11, it therefore reduces to two independent variables $\alpha_{v}$ and $\alpha_{s}$ that relate to homogeneous volume and pure shear deformations in the cell:

$$
\alpha_{i j k l}=\frac{1}{3}\left(\alpha_{v}-\alpha_{s}\right) \delta_{i j} \delta_{k l}+\frac{\alpha_{s}}{2}\left(\delta_{i k} \delta_{j l}+\delta_{i l} \delta_{j k}\right)
$$

Several cytoskeletal polarization mechanisms are possible with equation II.46, In their work, Zemel et al. study two extreme limits: the limit of axially induced polarization and the limit of purely orientational polarization. In the first scenario, stress fibers form or disassemble in the direction of local stress, whereas the stress fibers in the direction perpendicular to the local stress remain unaltered $\left(\alpha_{s}=\alpha_{v}\right)$. In the second 
scenario, the formation of new stress fibers in the direction of local stress is accompanied by a disassembly of stress fibers in the direction perpendicular to the stress. In dipole notation, this latter scenario corresponds to a mere alignment of dipoles in the direction of the elastic stress without changing the total number of dipoles or the magnitude of the single dipole moment, $[12]]\left(\alpha_{v}=0\right)$.

In mechanical equilibrium all stresses balance at the interface between cell and matrix:

$$
\sum_{j}\left(\sigma_{i j, c}^{r}-\sigma_{i j, m}^{r}\right) e_{j}=\sum_{j} \Delta\left\langle p_{i j}\right\rangle_{V} e_{j}
$$

With equations II.44 and II.45 the stress balance is consequently expressed as:

$$
\sum_{j}\left[\sum_{k l}\left(C_{i j k l, c} \Delta \epsilon_{k l, c}^{r}-C_{i j k l, m} \epsilon_{k l, m}^{r}[[13])\right] e_{j}=\sum_{j}\left(-\sum_{m n} \alpha_{i j m n} \sum_{k l} C_{m n k l, c} \Delta \epsilon_{k l, c}^{r}\right) e_{j}\right.
$$

where $\vec{e}$ is the unit vector normal to the surface of the interface between cell and matrix. If we now introduce an effective elastic tensor for the cell

$$
C_{i j k l, c}^{\mathrm{eff}}=C_{i j k l, c}+\sum_{m n} \alpha_{i j m n} C_{m n k l, c}
$$

equation II.48 takes the same form as the force balance equation of a passive elastic inclusion in an elastic medium[14],

$$
\sum_{j}\left(\sum_{k l} C_{i j k l, c}^{\mathrm{eff}} \Delta \epsilon_{k l, c}^{r}\right) e_{j}=\sum_{j}\left(\sum_{k l} C_{i j k l, m} \epsilon_{k l, m}^{r}\right) e_{j}
$$

Equation II.50 describes a known problem from solid state physics, the solution to which can be found in references [42, 14, 3, 64].

All equations that have been mentioned so far are also valid for the 2-D case of a cell on a flat matrix (substrate), in which the stresses perpendicular to the matrix surface are zero. Only small adaptations are necessary: the factor $1 / 3$ in equation II.46 is replaced by $1 / 2$. However, as Zemel et al. point out, the $2-\mathrm{D}$ version of the equations above is only valid if the matrix thickness is smaller than the cell size. Experimentally, this condition is often not met so that effectively a situation is encountered in the experiments that is in between the 2-D and the 3-D model presented here. As the 2-D and 3-D models show the same qualitative behavior of acto-myosin polarization as a response to matrix stiffness with perhaps slightly more prominent stress fibers in 2-D, the results of the models can nevertheless be used to explain experimental observations.

\footnotetext{
12 The conservation of total dipole moment in this scenario reflects constraints such as the limited amount of myosin motors or ATP in the cell.

13 Note that for the matrix the deformation in the reference frame, that is, just before the cell is brought into contact with the matrix, is zero.

14 The active elements in the cell therefore influence the effective elastic properties of the cell: for $\alpha_{i j m n}>0$ the cell appears to be more rigid. This means that if elasticity were to be measured for active cells, the obtained Young's modulus $E$ would be higher than any $E$ measured, for example, after myosin activity is blocked.
} 
To explain the particular experimental observation of the development of preferred orientations for stress fibers, some sort of anisotropy still needs to be considered in the model. Anisotropy can occur in the matrix, in the initial cell shape or even in the spreading behavior of the cell. An anisotropic spreading behavior would be caused, for example, by an anisotropic distribution of ligands on the matrix or by cell-type inherent properties. In their publication, Zemel et al. 65] concentrate on anisotropies induced by anisotropic cell shapes and by anisotropic spreading behavior. They include these anisotropies by specifying an aspect ratio $r(r \neq 1)$ for the ellipsoidal shape of the cell, where the long axis is chosen to be parallel to the $x_{1}$-axis, and by considering the initial stress $\sum_{k l} C_{i j k l, c} \epsilon_{k l, c}^{0}$ to be anisotropic in $x_{1}$-direction on the matrix surface. That is, they assume that $\epsilon_{k l, c}^{0}$ is such that

$$
\sum_{k l} C_{i j k l, c} \epsilon_{k l, c}^{0} \propto\left(\delta_{i j}+\eta \delta_{i 1} \delta_{j 1}\right)
$$

They find that for isotropic spreading $(\eta=0)$ the anisotropic cell shape leads to a nonmonotonic dependence of stress fiber polarization on matrix rigidity (see curves with $\eta=0$ in figure II.5 a) and b)). For very soft and very rigid matrices the stress fiber polarization approaches zero, whereas for intermediate rigidities a peak is observed in stress fiber polarization. This phenomenon for rigid matrices can be explained by the fact that the active stresses $\left\langle p_{i j}^{0}\right\rangle_{V}$ in the cell are opposed by isotropic, equal and opposing stresses in the matrix so that the symmetry is not broken. For very soft matrices, the lack in stress fiber polarization is explained by the break-down of the feedback system in equation II.45: if the matrix is very compliant, the elastic stress in the cell is reduced and consequently the force dipole development is small.

In contrast, anisotropic spreading introduces a contribution that grows monotonically with matrix rigidity (see figure II.5 a) and b)). Due to this contribution, which is most noticeable in the limit of orientational polarization for the polarizability tensor (equation II.46), stress fiber polarization is present even on very rigid substrates, such as glass. 


\section{Axially Induced Polarization}

a)

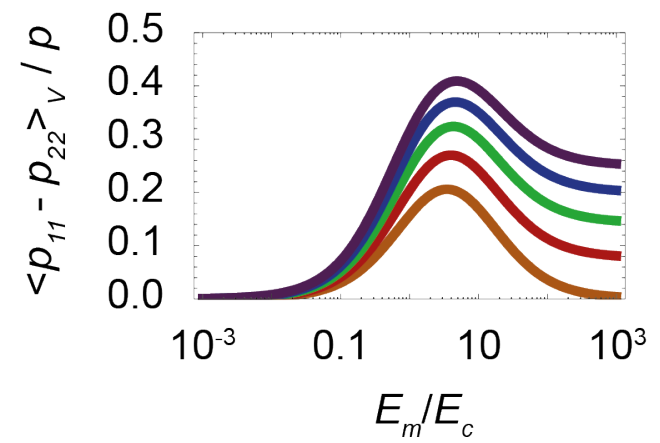

c)

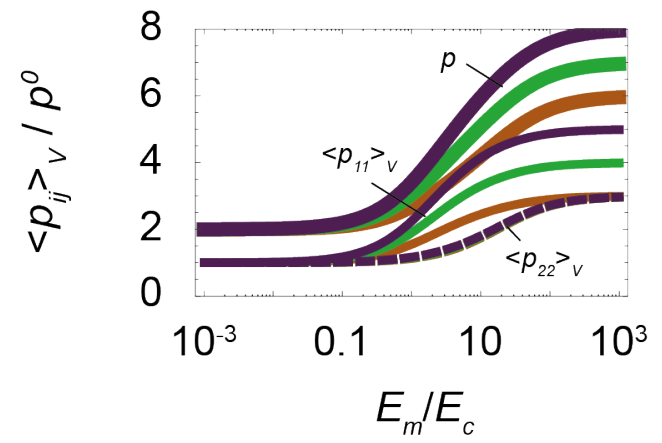

Orientational Polarization

b)

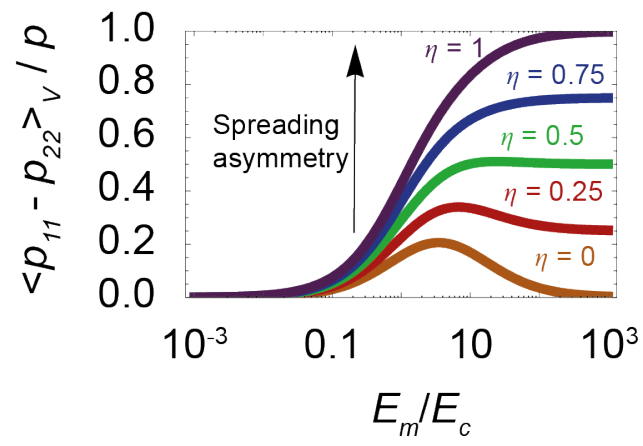

d)

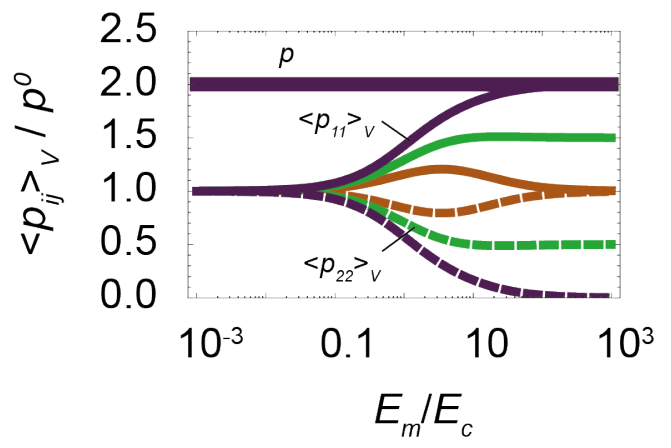

Figure II.5: Modeling stress fiber formation - influence of matrix rigidity, initial cell shape and cell spreading behavior: a) and b) Stress fiber orientation as a function the matrix's Young's modulus $E_{m}$ and the spreading asymmetry parameter $\eta$ during 2-D spreading of an ellipsoidal cell with aspect ratio $r=3(\eta=0$ : isotropic cell spreading). For normalization purposes, $E_{m}$ is divided by the Young's modulus of the cell, $E_{c}$. The orientation of stress fibers is indicated by the order parameter $\left\langle p_{11}-p_{22}\right\rangle_{V} / p$ for the average force dipole tensor $\left\langle p_{i j}\right\rangle_{V}$ in the cell, where $p:=\left\langle p_{11}\right\rangle_{V}+\left\langle p_{22}\right\rangle_{V}$. Two polarization mechanisms are considered: axially induced polarization $(\mathrm{a}, \mathrm{c})$ and orientational polarization $(\mathrm{b}, \mathrm{d})$. The following settings were chosen for the components $\alpha_{v}$ and $\alpha_{s}$ of the polarizability tensor in the cell: $\alpha_{s}=\alpha_{v}=2(\mathrm{a}, \mathrm{c})$ and $\alpha_{s}=2, \alpha_{v}=0(\mathrm{~b}, \mathrm{~d})$. Images c) and d) display the different components of the average force dipole tensor for the same scenarios plotted in a) and b) with matching colors. The component $\left\langle p_{11}\right\rangle_{V}$ is marked with thin lines, $\left\langle p_{22}\right\rangle_{V}$ is marked with dashed lines and $p$ is represented by a bold line. All tensor components are normalized with the average force dipole moment per unit volume $p^{0}$ in the initial state of the cell before stress fibers are formed. The Poisson ratio of the cell was set to $\nu_{c}=0.3$ and the Poisson ratio of the matrix was set to $\nu_{m}=0.45$. [Figure from [65], labels were adapted] 


\section{Materials and Methods}

\section{III.1 Subjects and Platelet Isolation}

Experiments were conducted in agreement with the ethical vote of the Ethic Committee of the University of Göttingen, votum 11/11/09, which regulates how research is to be performed on human subjects or human tissue samples. Platelets were collected at the Blood Donation Center of the University Clinic of Göttingen from healthy, volunteer donors that have not taken any anti-platelet or pain killer medication at least 7 days before donation. Platelets were harvested at the Clinic using the apheresis system of Trima Accel (Caridian BCT Europe, Garching, Germany), or prepared from buffy coats pooled from 4 donors with the same AB0 blood group using Fenwal blood donation bags with the blood stabilizer, citrate-phosphate-dextrose (CPD), and an automated blood separation system, Fenwal OptiPress II (Fenwal Europe SPRL, Mont-SaintGuibert, Belgium). Leucocyte-depleted platelet concentrates were stored at $22-24^{\circ} \mathrm{C}$ under continuous, gentle agitation for at least 3 days. Platelet isolation from platelet concentrates and subsequent experiments were conducted between day 4 and 7 after donation, which was after clinical expiration of the concentrates, but within the platelets' average life span of $7-10$ days [38, p. 45].

Before platelets were isolated from a concentrate, we warmed the buffer solution, pipes-saline-glucose (PSG)(5 mM PIPES, $145 \mathrm{mM} \mathrm{NaCl}, 4 \mathrm{mM} \mathrm{KCl}, 50 \mu \mathrm{M} \mathrm{Na}_{2} \mathrm{HPO}_{4}$, $1 \mathrm{mM} \mathrm{MgCl} 2 \cdot 6 \mathrm{H}_{2} \mathrm{O}, 5.5 \mathrm{mM}$ glucose; $\mathrm{pH}$ 6.8), and the medium, M199 (with Earle's balanced salt solution, L-glutamine and $25 \mathrm{mM}$ HEPES, Lonza, Basel, Switzerland) up to $37^{\circ} \mathrm{C}$ and saturated them with $\mathrm{CO}_{2}$ in an incubator. Next, we retrieved the first $2 \mathrm{ml}$ of platelet concentrate and directly discarded them to remove any contamination that was potentially introduced at the opening of the platelet bag. Then we retrieved $4 \mathrm{ml}$ and transferred them to a falcon tube by gently letting the suspension run down the tube wall to prevent the cells from getting mechanically stressed. Aggregationinhibiting prostaglandin $\mathrm{PGE}_{1}$ (Cayman Chemical Company, Ann Arbor, MI, USA) was added at a final concentration of $2.6 \mu \mathrm{g} / \mathrm{ml}$ and we performed three centrifugation steps, each of $20 \mathrm{~min}$ at $480 \times \mathrm{g}$ and $21-22^{\circ} \mathrm{C}$. Between centrifugation steps, we removed the supernatant and carefully resuspended the pellet in $4 \mathrm{ml}$ warm PSG, to which we added $\mathrm{PGE}_{1}$ as before. After the final centrifugation step, we resuspended the pellet in $1 \mathrm{ml}$ warm M199 and counted cells using a heparinized Micro-Hematocrit Capillary (Fisherbrand, Hampton, NH, USA) and a ZIPocrit Microhematocrit Centrifuge Reader Card (LW Scientific, Lawrenceville, GA, USA). The cell suspension was then diluted to final platelet counts of $5-9 \cdot 10^{7}$ cells $/ \mathrm{ml}$. 


\section{III.2 Preparation of Substrates}

Polyacrylamide (PAA) substrates were prepared on cover slips by polymerizing monomeric acrylamide mixed with the crosslinker bis-acrylamide in a protocol adapted from references [49] and [5]. Before the polyacrylamide substrates were prepared, however, the surface of the cover slips was pretreated to achieve covalent bonding between cover slip and polyacrylamide substrate [11].

For that purpose, we cleaned the surface of the cover slips $(24 \times 60 \mathrm{~mm}$, No. 1 or $22 \times 22$, No. 0) and applied a thin layer of $0.1 \mathrm{~N} \mathrm{NaOH}$, which was allowed to dry. Next, the glass was treated with 3-aminopropyltrimethoxy silane (97\% Sigma Aldrich, St. Louis, MO, USA), which we left on the cover slips for 5 min, before we thoroughly washed the surface with MilliQ water (ultrapure water with a level of total organic carbon (TOC) of $<10 \mathrm{ppb}$ and a content of microorganism of $<0.1 \mathrm{cfu} / \mathrm{ml}$, obtained from a MilliQ Synth A10 Academic System with a resistivity of $18.2 \mathrm{k} \Omega$, Billerica, MA, USA) and left it to dry. By these means covalent Si-O-Si-bonds were established between the silica in the glass and the silane, leaving the amine group of the silane exposed at the surface [2, 23] (see also figure [II.1). As soon as the cover slips were dried, we applied a solution of $0.5 \%$ glutaraldehyde $(70 \%$ glutaraldehyde stock solution, Polysciences, Warrington, PA, USA) in phosphate buffered saline (PBS; $137 \mathrm{mM} \mathrm{NaCl}, 2.7 \mathrm{mM} \mathrm{KCl}, 4.3 \mathrm{mM} \mathrm{Na}_{2} \mathrm{HPO}_{4} \cdot 12 \mathrm{H}_{2} \mathrm{O}, 1.4 \mathrm{mM} \mathrm{KH}_{2} \mathrm{PO}_{4}$ ) to the cover slips and left it to react for $30 \mathrm{~min}$. The surface of the cover slips was then again thoroughly washed with MilliQ water and left to dry. As a result of the glutaraldehyde treatment, the exposed amine group of the silane was covalently bound to one aldehyde group of the glutaraldehyde. The remaining part of the glutaraldehyde was left free to react both with other glutaraldehyde molecules and later on with the acrylamide [47].

Having prepared the cover slips, we mixed the acrylamide polymerization solution with a final composition of: $8 \%$ monomeric acrylamide $(40 \%$ stock solution, Bio-Rad, Hercules, CA, USA); $0.04 \%$ crosslinker bis-acrylamide (2 \% stock solution, Bio-Rad); 50 mM HEPES (99.5 \%, Carl Roth, Karlsruhe, Germany); 13.4 mM catalyzer N,N,N',N'- tetramethylethylenediamine (TEMED, Bio-Rad), $10 \mathrm{mM}$ acrylic acid N-hydroxysuccinimide ester (NHS ester, Sigma Aldrich) and $2 \mu \mathrm{l} / \mathrm{ml}$ red fluorescent marker beads (carboxylate-modified microspheres, $0.1 \mu \mathrm{m}$ diameter, $2 \%$ solids, Invitrogen, Carlsbad, CA, USA). In a first step to prepare the polymerization solution, we mixed the acrylamide, bis-acrylamide, HEPES and TEMED, and set the $\mathrm{pH}$ to 6 by addition of $\mathrm{HCl}$. Here, it is important to set the $\mathrm{pH}$ accurately, as a lower $\mathrm{pH}$ will inhibit polymerization and a higher $\mathrm{pH}$ can hydrolyze the NHS ester [49. Having set the $\mathrm{pH}$, we added the NHS ester and the fluorescent beads and diluted the solution with MilliQ water to the above mentioned final concentrations of the polymerization solution. We then manually degassed the solution using a syringe, while keeping the gap between the syringe and the beaker sealed with parafilm. Finally, we started polymerization by the addition of ammonium persulfate (Bio-Rad) at a final concentration of $0.05 \%$. We added $5 \mu \mathrm{l}$ drops of the solution quickly to the pretreated cover slips and immediately covered them by round cover slips (10 mm diameter, No.1) to obtain flat substrates with a nominal height of $\sim 64 \mu \mathrm{m}$ (figure III.2). We turned the assembly upside down during polymerization, so that the fluorescent beads would sink down and 

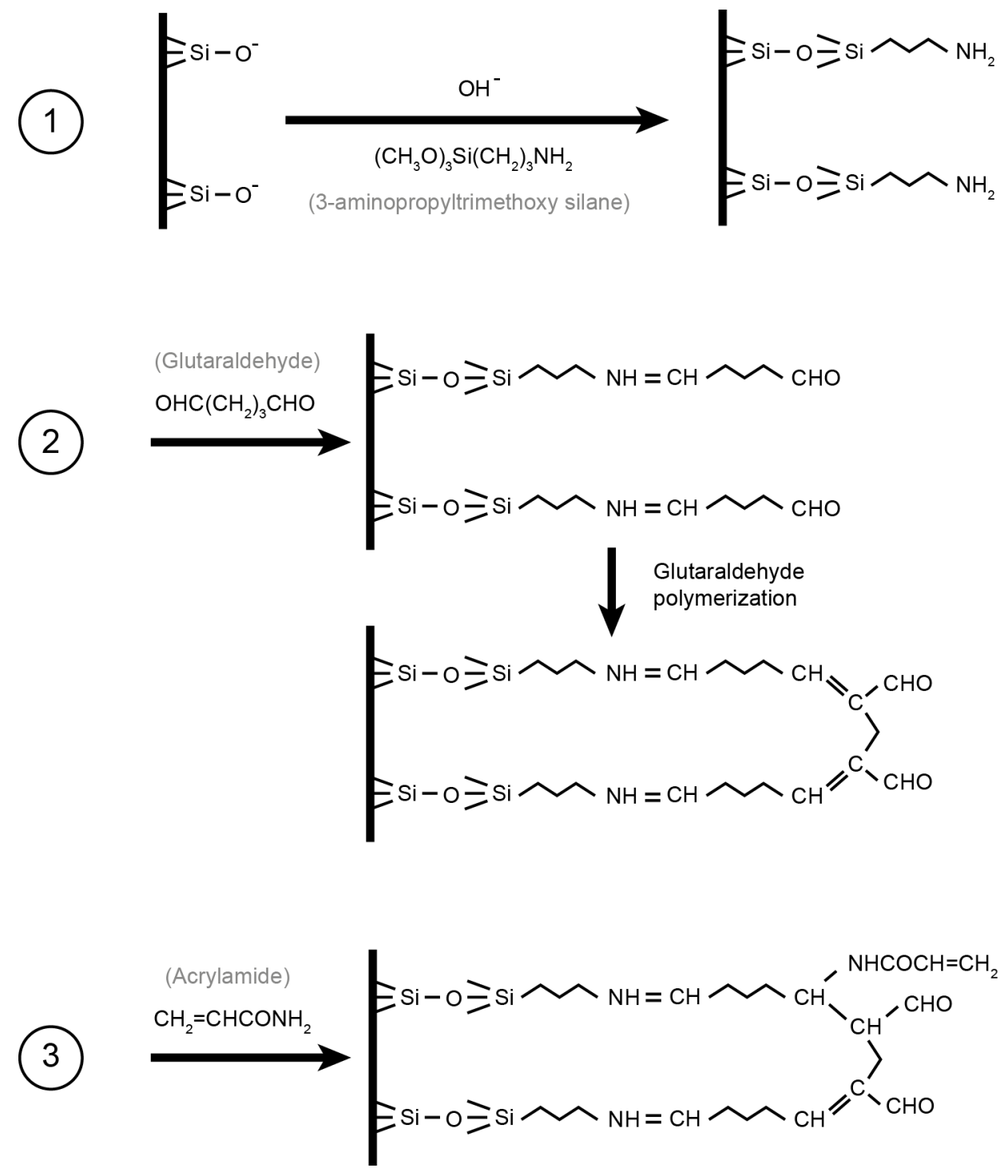

Figure III.1: Sketch of the chemical reactions taking place in order to covalently attach polyacrylamide (PAA) substrates to glass cover slips: Glass cover slips were treated with $\mathrm{NaOH}$ and 3-aminopropyltrimethoxy silane to establish Si-O-Si-bonds between the silica in the glass and the silane. Then a glutaraldehyde solution was applied to the cover slips, which was left to react with the silane. Glutaraldehyde furthermore polymerized and in its polymerized form reacted with the acrylamide, which was finally added to the cover slips. [Figure based on sketches in [2, 47]] 
gather close to the round cover slip. This way, we ensured that the beads would be located within the topmost layer of the final PAA substrate.

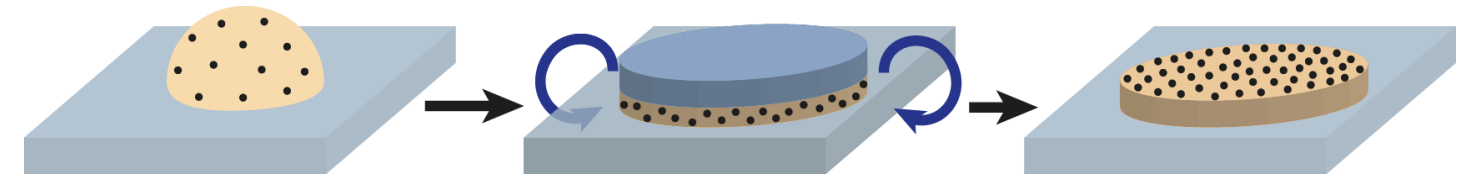

Figure III.2: Sketch of the last steps during substrate preparation: Bead-containing acrylamide polymerization solution was added to a cover slip. A second, smaller and round cover slip was placed on top of the drop of solution to flatten it. The assembly was then turned upside down, so that beads can accumulate close to the surface of the round cover slip while polymerization proceeds. After one hour, the round cover slip was removed, revealing the underlying substrate, which at this final stage contained a high-density bead layer at the top surface.

After one hour of polymerization time, we covered the substrates with PBS to better be able to remove the round cover slips with a pair of tweezers. The flat, crosslinked substrates were then rinsed with MilliQ water and a fibrinogen protein layer was covalently bound to the surface via the NHS ester by incubation at $4{ }^{\circ} \mathrm{C}$ overnight with $0.1 \mathrm{mg} / \mathrm{ml}$ protein solution (human plasma, diluted in MilliQ water or PBS from a stock solution of $20 \mathrm{mg} / \mathrm{ml}$ in $0.9 \% \mathrm{NaCl}$, Calbiochem - Merck KGaA, Darmstadt, Germany). On the following day, we rinsed the substrates again with MilliQ water and removed the remaining hydrolyzed NHS ester with $50 \mathrm{mM}$ ethanolamine solution (99\%, diluted in $50 \mathrm{mM} \mathrm{HEPES} \mathrm{or} \mathrm{MilliQ} \mathrm{water,} \mathrm{Carl} \mathrm{Roth),} \mathrm{which} \mathrm{we} \mathrm{left} \mathrm{to} \mathrm{react}$ for one hour at room temperature. Substrates were again rinsed with and stored in MilliQ water. Shortly before experiments, the substrates were washed 3 times with M199 to fully saturate the substrate with cell medium. Fluorescence images taken during experiments revealed that the bead density at the surface of the substrate is such that the average bead distance is $\sim 0.8 \mu \mathrm{m}$ (see also section IV.6).

\section{III.3 Substrate Elasticity Measurements}

The substrate elasticity was measured using a Physica MCR 501 rheometer (Anton Paar, Graz, Austria). To that end, we prepared an acrylamide polymerization solution as described in section III.2 with the exception of the fluorescent beads. The ammonium persulfate was added shortly before the rheology measurement (concentration as in section III.2) and $490 \mu \mathrm{l}$ of the solution were immediately applied to a round cover slip (25 mm diameter, pretreatment as in section III.2 - instead of applying $\mathrm{NaOH}$, the cover slip was put into a plasma cleaner and only $\sim 4 \% 3$-aminopropyltrimethoxy silane solution was used). We recorded the polymerization process every $30 \mathrm{~s}$ over 2 hours using a flat top plate (25 mm diameter) at a frequency of $1 \mathrm{~Hz}$ and an amplitude of deformation of $1 \%$. Following the time record, we performed a frequency sweep from $0.01 \mathrm{~Hz}$ to $100 \mathrm{~Hz}$ at an amplitude of $1 \%$, which was succeeded by an amplitude sweep from $0.01 \%$ to $500 \%$ at a frequency of $1 \mathrm{~Hz}$. 
The output of the rheometer, the shear modulus $\mu$, was converted to elasticity values with the following equation [37, p. 132]

$$
E=2 \mu \cdot(1+\nu)
$$

where $E$ is the elastic modulus or Young's modulus and $\nu$ is the Poisson ratio, which is assumed to be $0.3^{[1]}$ for polyacrylamide substrates.

We determined the elasticity of the PAA substrate by fitting a constant to the region of saturation in the time track and the linear regions in the frequency track and amplitude track (see example data set in figure III.3). For our substrates we thus obtained a Young's modulus of $\sim 4 \mathrm{kPa}$.

a)
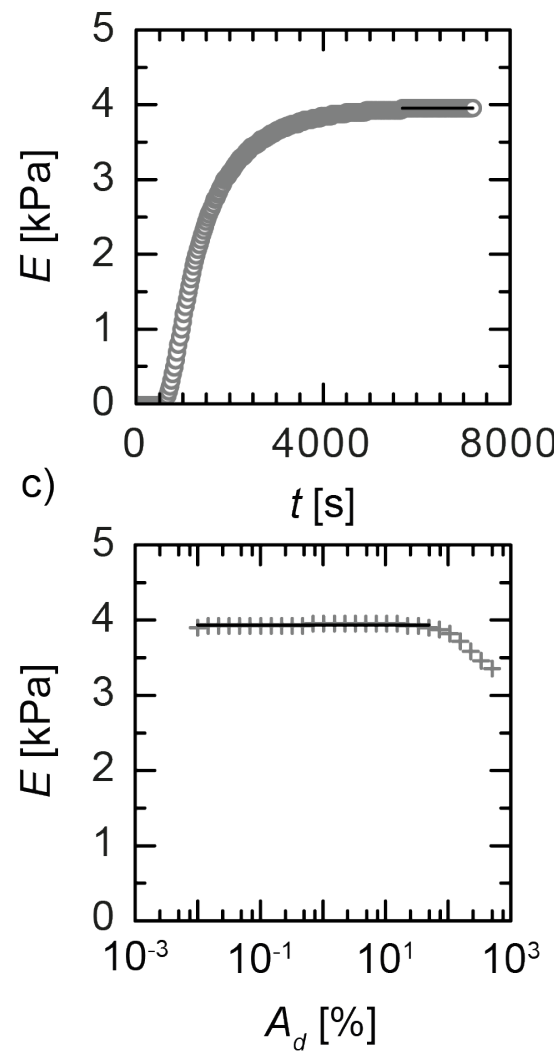

b)

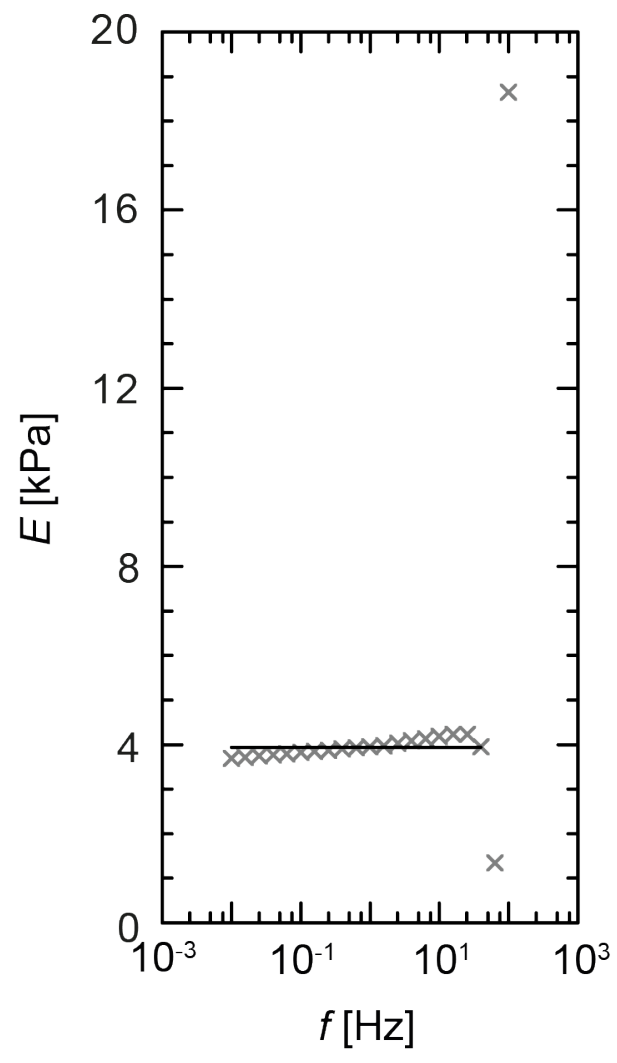

Figure III.3: Characterization of substrate elasticity by rheology: a) Elasticity curve of the substrate polymerization process (open circles) measured with a flat top rheometer plate with an oscillation frequency of $1 \mathrm{~Hz}$ and an amplitude of deformation of $1 \%$, b) Frequency sweep from $0.01 \mathrm{~Hz}$ to $100 \mathrm{~Hz}$ at an amplitude of $1 \%$ (crosses), c) Amplitude sweep from $0.01 \%$ to $500 \%$ at a frequency of $1 \mathrm{~Hz}$ (pluses). The flat regions in plots a)-c) were fitted by a constant (black lines).

\footnotetext{
${ }^{1}$ according to indications in the manual for the LIBTRC program written by Prof. Micah Dembo (see also section IV.4 page 71)
} 


\section{III.4 Traction Force Microscopy (TFM)}

During a TFM recording, platelet solution $\left(5-9 \cdot 10^{7}\right.$ cells $\left./ \mathrm{ml}\right)$ was applied to fibrinogencoated, elastic PAA substrates in a stage top incubator (Tokai Hit, Shizuoka-ken, Japan) with a humid atmosphere containing $5 \% \mathrm{CO}_{2}$ at $37^{\circ} \mathrm{C}$. To initiate platelet activation thrombin (from human plasma, Sigma Aldrich) was added to the cells at a final concentration of $1 \mathrm{u} / \mathrm{ml}$. As soon as the platelets started contracting, we recorded the resulting substrate deformation via fluorescent marker beads within the substrates (see figure I.13). Beads were traced over a total time period of $60-92 \mathrm{~min}$ by epifluorescence microscopy at $100 \times$ magnification using an inverted light microscope (see section III.6) with 10 s intervals between consecutive images. Simultaneously, we recorded phase contrast images (see again section III.6) to obtain the cellular boundary and to identify the cell-substrate contact area. Having recorded these images, we measured the height of the PAA substrates. For that purpose, we first focused on a bead layer that formed at the bottom of the PAA substrate during substrate preparation and recorded the position of its focal plane. We next focused on the bead layer at the top of the substrate and noted down its position. The difference of the two focal planes was then taken to be the substrate height. From the images obtained and the measured height of the substrates, traction force fields were calculated with a spatial resolution of $0.2-0.3 \mu \mathrm{m}$ (see section IV.6).

\section{III.5 Platelet Staining}

The fact that platelets haven't got a nucleus makes live imaging of cytoskeletal reorganization steps during platelet activation challenging. Thus, platelets cannot be transfected to express fluorescent proteins by standard methods. Instead, we had to opt for an approach, where we fix platelets at different stages of activation and stain them for filamentous actin (F-actin) with a dye-conjugated form of the toxin phalloidin. By these means, we were able to observe actin reorganization in platelets that were activated on the soft PAA substrates. As an additional control we repeated the same experiment on glass surfaces, which are considerably stiffer than PAA and on which platelets are known to develop distinct actin stress fibers [54]. Finally, we used immunofluorescence staining to image the distribution of myosin motors and focal adhesion sites in platelets that were fully activated on PAA.

\section{III.5.1 Staining on PAA}

To image actin reorganization on PAA substrates, platelet solution $\left(6 \cdot 10^{7}\right.$ cells $\left./ \mathrm{ml}\right)$ was added to the substrates and left to settle for $15 \mathrm{~min}$. Then, thrombin solution was added at a final concentration of $1 \mathrm{u} / \mathrm{ml}$ to synchronize and trigger platelet activation. After an activation time of 2.5, 5, 10, 20 or $30 \mathrm{~min}$, we washed substrates twice (or once in the case of an activation time of $2.5 \mathrm{~min})$ with warm $\mathrm{PBS}\left(37^{\circ} \mathrm{C}\right)$. For this and all subsequent washing procedures, we left the washing solution on the substrates for about 5 min between washing steps, to thoroughly remove reagents. We then fixed the cells with $4 \%$ paraformaldehyde solution at room temperature, which we left on the 
substrates for $20 \mathrm{~min}$, before they were washed again thrice with PBS. Substrates were stored overnight at $4^{\circ} \mathrm{C}$.

On the following day, we incubated the substrates with $0.5 \%$ Triton-100 for $10 \mathrm{~min}$ at room temperature to permeabilize the cells, which was washed out afterwards three times with PBS as before. Unspecific binding sites in the cells were blocked with $10 \%$ goat serum for $30 \mathrm{~min}$ at room temperature and substrates were washed again twice with PBS-1 \% goat serum. We then incubated substrates for $30 \mathrm{~min}$ at room temperature with Alexa Fluor ${ }^{\circledR}$ phalloidin 488 (Excitation/Emission wavelength: $\sim 495 / \sim 518 \mathrm{~nm}$, Invitrogen) diluted in PBS-1 \% goat serum to a final concentration of $5 \mathrm{u} / \mathrm{ml}$. During incubation with phalloidin, substrates were covered with aluminum foil to avoid bleaching. Finally, substrates were washed three times with PBS and mounted on microscope slides (see figure III.4) with ProLong ${ }^{\circledR}$ Gold antifade solution (Invitrogen). For that purpose, the cover slips with the substrates were placed on microscope slides, which were slightly wet so that the cover slips attached to them due to capillary forces. 8-10 $\mu \mathrm{l}$ mounting solution was pipetted onto and along the edge of the substrates, the assembly was covered with another cover slip (diameter $15 \mathrm{~mm}$, No. 1) and fixed with 4 droplets of transparent nail polish. The mounting solution was left to harden in darkness first for 45-60 min at room temperature and then for 3 days at $4^{\circ} \mathrm{C}$. Before placing the substrates in the fridge, however, the edges of the assembly were completely sealed with nail polish to ensure that the mounting solution is no longer exposed to air. The samples were looked at from the top, that is, the assembly was turned upside down on the microscope, to avoid optical distortions caused by the PAA substrate.

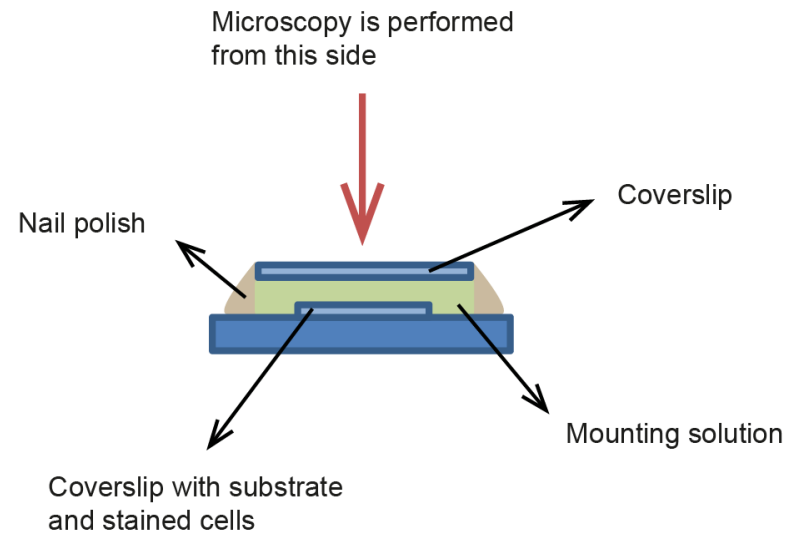

Figure III.4: Sketch of mounted sample with activated platelets that were stained for cytoskeletal F-actin, vinculin (focal adhesions) or myosin IIA motor proteins. Microscopy is performed by turning the assembly upside down and looking at the platelets from top to avoid distortions arising from looking through the substrate.

Additionally to recording the actin reorganization during platelet activation, we visualized the sites of force generation and transduction in the final stages of platelet activation by staining both the platelet myosin IIA motors as well as vinculin, a protein, which is contained in focal adhesion sites. These two components were looked at in two separate experiments. In the first experiment, we labeled both vinculin and actin. The second experiment consisted of a triple staining of vinculin, actin and myosin. The 
protocol we followed for both experiments is similar to the above mentioned with a few modifications. Thus, platelets were activated for $60 \mathrm{~min}$ to allow for complete activation and following the washing steps after blocking unspecific binding sites $[2]$, platelets were incubated overnight at $4^{\circ} \mathrm{C}$ with the primary antibody to vinculin (monoclonal antibody from mouse, abcam, Cambridge, UK) diluted in PBS-1 \% goat serum to a final concentration of $2 \mu \mathrm{g} / \mathrm{ml}$. During triple staining, the primary antibody to nonmuscle myosin IIA (polyclonal antibody from rabbit, abcam) was furthermore added to the platelets at a final concentration of $2 \mu \mathrm{g} / \mathrm{ml}$ before incubation overnight. Afterwards, substrates were washed three times with PBS-1 \% goat serum, then incubated once more with $0.5 \%$ Triton-100 for about $10 \mathrm{~min}$ and washed again three times with PBS-1 \% goat serum. The secondary antibody to vinculin, which was conjugated to the dye Alexa Fluor ${ }^{\circledR} 568$ (goat anti mouse, Excitation/Emission wavelength: $\sim 578 / \sim 603$ nm,

Invitrogen), was applied to the cells at a final concentration of $5 \mu \mathrm{g} / \mathrm{ml}$. For triple staining, we furthermore added the secondary antibody to myosin, conjugated to Marina blue ${ }^{\circledR}$ (goat anti-rabbit, Excitation/Emission wavelength: $\sim 365 / \sim 460 \mathrm{~nm}$, Invitrogen), at a final concentration of $10 \mu \mathrm{g} / \mathrm{m}[[3]$. Secondary antibodies were left to react for 2 hours at room temperature under an aluminum foil to avoid bleaching. In the end, phalloidin was added to stain filamentous actin and the final washing and mounting occurred as described above with the small difference that the first hardening of the mounting solution at room temperature for the vinculin-actin-staining experiment prevailed for 63-68 min. Also, the final washing for the triple staining was adjusted to the increased amount of antibodies, which was used there, by extending it to include 5 washing steps.

It should be noted that for the triple staining experiment, cell solutions evaporated a little during platelet activation at the very beginning of the staining protocol. However, images obtained by epifluorescence microscopy (section III.6) for both vinculin and actin in this staining experiment resembled those from the double staining (section V.2.2) so that we assume the influence of evaporation to have been small.

\section{III.5.2 Control Staining on Glass}

Traditionally, platelet staining experiments have been carried out on glass surfaces [54]. As a control, we have therefore repeated the actin staining on glass, choosing as platelet activation times: 2.5, 5, 10, 15 and $30 \mathrm{~min}$. To see whether bead uptake by platelets would correlate with cytoskeletal reorganization, we furthermore added a solution of yellow-green fluorescent beads to the cells (diameter of $0.2 \mu \mathrm{m}$, Invitrogen). Cell anchorage to glass was provided by a fibrinogen coat on the cover slips. This coat was applied to the glass by incubating the cover slips with $0.1 \mathrm{mg} / \mathrm{ml}$ human fibrinogen solution (Calbiochem, La Jolla, CA, USA) over 30 min at $37^{\circ} \mathrm{C}$. Afterwards, unspecific binding sites were blocked with $1 \%$ human serum albumin $(25 \%$ stock solution diluted in Hank's balanced salt solution (HBSS); CLS Behring, King of Prussia, PA, USA),

\footnotetext{
${ }^{2}$ During triple staining of myosin, actin and vinculin unspecific binding sites were blocked for 43 min instead of the usual 30 min.

${ }^{3}$ For triple staining we also performed a control experiment for the secondary antibodies, the result of which looked normal.
} 
which was left to react on the cover slips for again 30 min at $37^{\circ} \mathrm{C}$. Cover slips were then rinsed three times with HBSS (without phenol red, Lonza, Basel, Switzerland) prior to experiments.

As staining experiments on glass have been performed during a visit to one of our collaborating labs (University of Utah, Salt Lake City, UT, USA), some details of the staining procedure described in the previous section were altered here. Thus, platelet activation was triggered with $0.1 \mathrm{u} / \mathrm{ml}$ thrombin and cells were fixed directly after activation, with no washing in between, by adding paraformaldehyde at a final concentration of $1 \%$ and leaving it to react for $20 \mathrm{~min}$. Following fixation, cells were washed three times with HBSS. Then Alexa Fluor ${ }^{\circledR}$ phalloidin 555 (Excitation/Emission wavelengths: $555 / 565 \mathrm{~nm}$, Invitrogen) was applied to the cells at a final concentration of $7.3 \mathrm{u} / \mathrm{ml}$ without prior permeabilization or blocking of unspecific binding sites. After $30 \mathrm{~min}$ of incubation with the phalloidin, cells were again washed three times with HBSS and imaged using a confocal microscope (section III.6).

\section{III.6 Microscopy}

All experiments performed on PAA substrates have been imaged at a fully motorized, inverted light microscope (IX81, Olympus, Tokio, Japan). This microscope was equipped for phase contrast imaging and epifluorescence imaging in a whole range of magnifications (10-100x). Both imaging techniques are designed to make transparent objects, such as biological samples, visible under a microscope. In addition, fluorescence microscopy enables smallest objects to be observed even when their size is below the wavelength of visible light. This is achieved by tagging selected components of a sample with fluorescent dyes prior to experiments. Under the microscope these dyes are then excited at selected wavelengths and consequently begin to fluoresce. In our setup, for example, selective excitation is accomplished by directing the light provided by a divergent, broad spectrum Xenon arc lamp $(150 \mathrm{~W})$ at a collimator and a filter cube (figure III.5). While the collimator ensures homogeneous illumination, an excitation filter in the filter cube is transparent only to light within a small band of excitatory wavelengths. The light that passes the excitation filter is afterwards reflected at a dichroic mirror in the filter cube. Subsequently, it is focused on the sample by an objective lens (in our experiments: 100x oil immersion objective, UPLFLN 100XO2PH, with a numerical aperture of 1.3 and a working distance of $0.2 \mathrm{~mm}$ ), hence exciting the dye in the sample. Following excitation the dye molecules re-emit light with slightly larger wavelengths due to the so called Stokes shift. Re-emitted light is transmitted by the dichroic mirror on the way back, passing through an emission filter in the filter cube with a wavelength cut off, so that any light not originating from the sample gets blocked. Finally, the emitted light is focused onto a CCD camera (water-cooled ORCA-R2 C10600-10B camera, QE >70\%, 1.3 MP with pixel size $6.45 \mu \mathrm{m} \times 6.45 \mu \mathrm{m}$, 16.2 fps, Hamamatsu Photonics Deutschland GmbH, Herrsching am Ammersee, Germany). Two filter cubes have been used in our experiments. One is a single band filter cube, selective for excitation wavelengths around $480 \mathrm{~nm}$ (filter BP470-495) and emission wavelengths of $\sim 530 \mathrm{~nm}$ (filter BA510-550). The matching dichroic mirror (DM505), in turn, transmits any wavelength above $505 \mathrm{~nm}$. The other filter cube used, 
is a triple band filter cube (83000v2 DAPI/FITC/Texas Red), which is optimized for excitation/emission wavelengths around: 350, 492, $572 \mathrm{~nm} / 462,531,628 \mathrm{~nm}$.

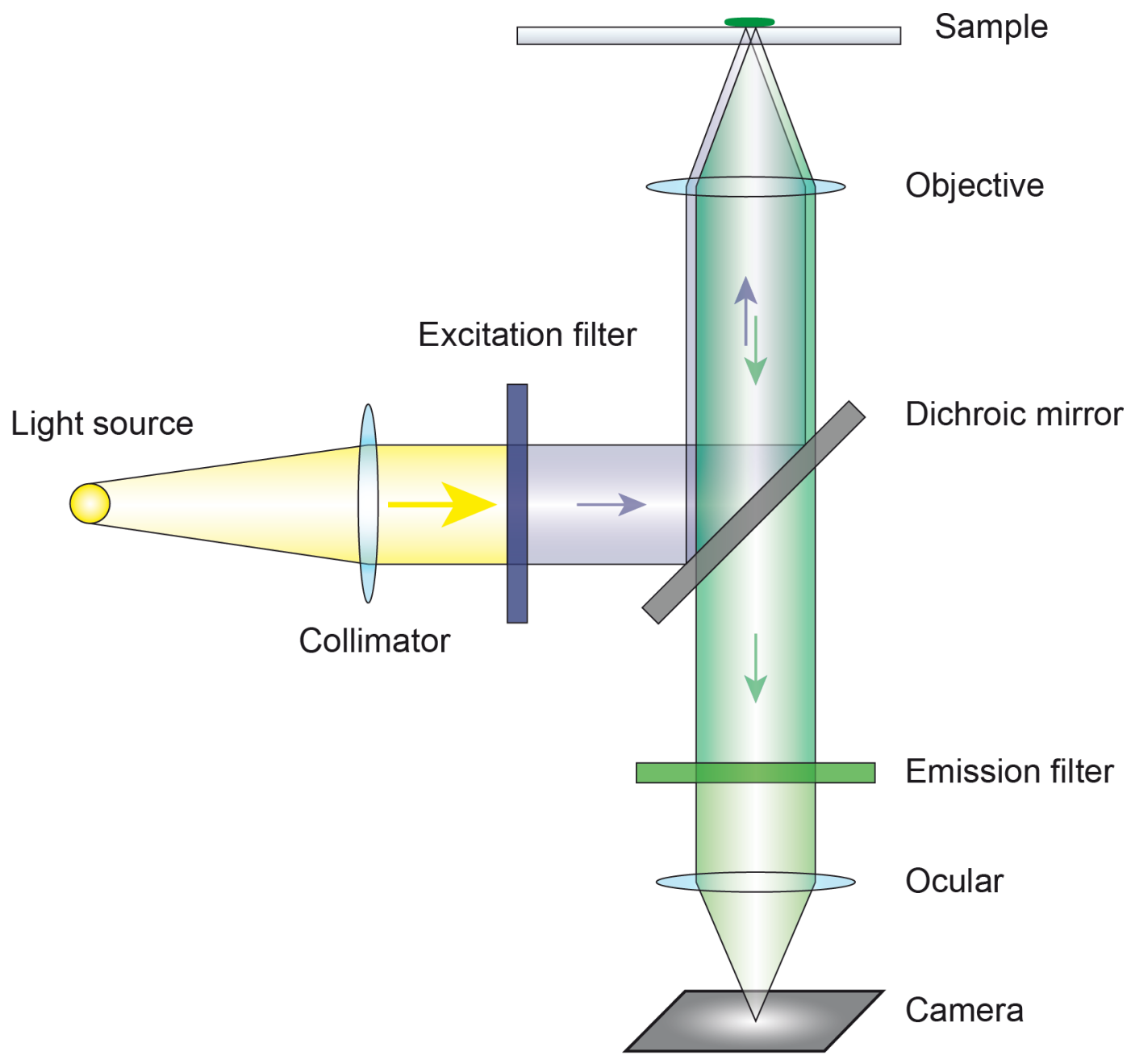

Figure III.5: Sketch of an epifluorescence microscope: A specimen, labeled with a fluorescent dye, is imaged during epifluorescence microscopy by exciting the dye in the sample at selected wavelengths and collecting the emitted fluorescence. To obtain a clear signal, the emitted light from the sample is passed through a filter specific to the emitted wavelengths. [Figure by Jens Nolting]

In addition to using fluorescence microscopy in our experiments, we have taken phase contrast images of our cells. Phase contrast imaging takes advantage of the fact that samples diffract light and retard its phase by small amount:[4] (about $\lambda / 4$ ). In our setup, for example, a ring slit in the so called condenser filters out a ring of light from

\footnotetext{
${ }^{4}$ Phase shifts are small only, if the sample is thin. As cellular samples do have small thicknesses, phase contrast imaging is widely used for biological applications.
} 


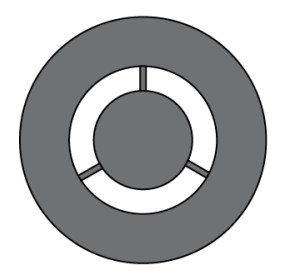

Condenser ring slit

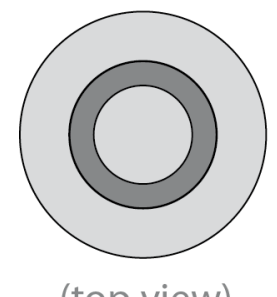

(top view)

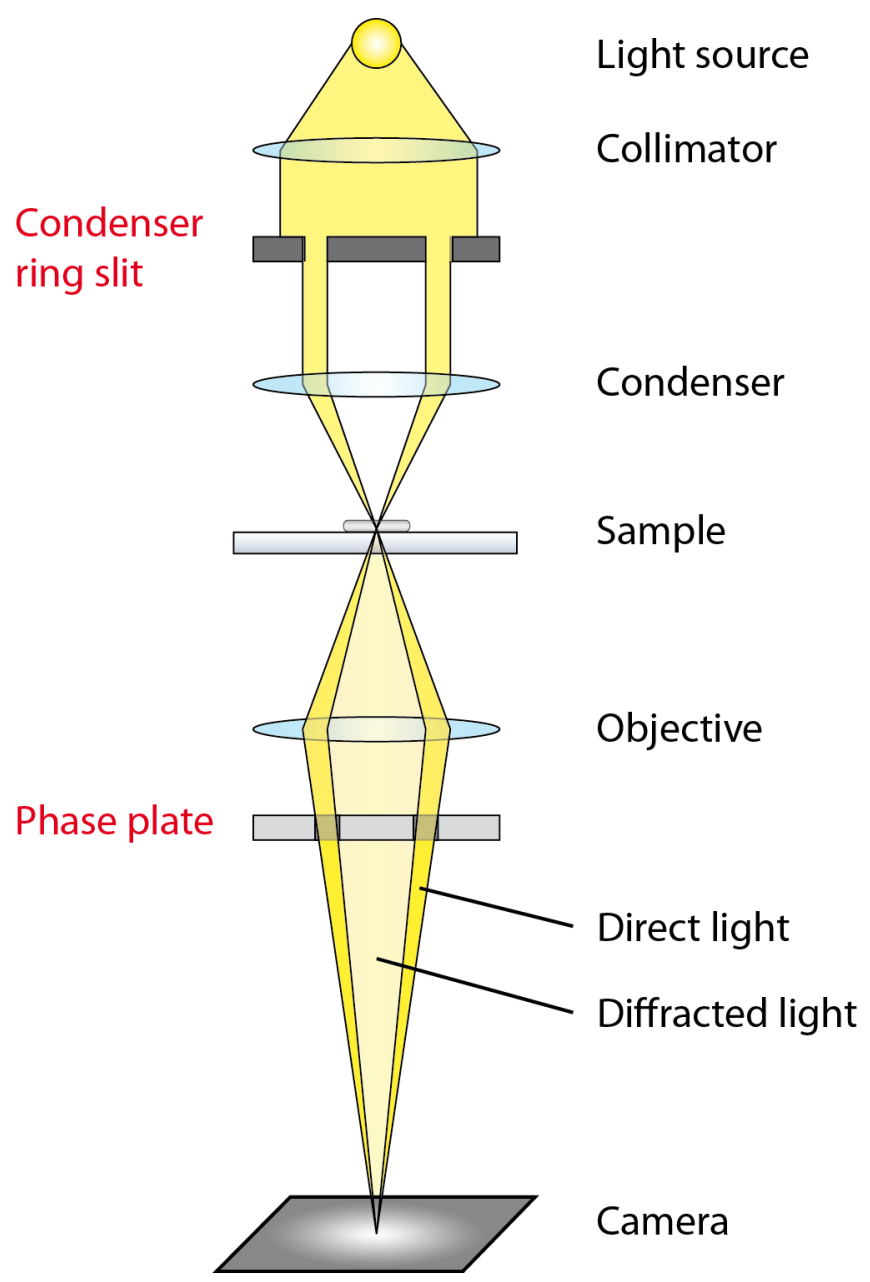

Figure III.6: Sketch of phase contrast microscopy: Diffracted and phase-shifted light from an illuminated sample is overlaid in front of a camera with direct light from the primary beam. This direct light, in turn, has been shifted in phase by $\lambda / 4$ using a phase plate. The superposition of light from the two sources leads to constructive and destructive interference, creating a high contrast image of the sample. [Figure by Jens Nolting]

the Xenon lamp illumination, which then impinges on our sample (figure III.6). Here, part of the light passes through the sample undeviating and the rest is diffracted, which also introduces a phase shift of about $\lambda / 4$. The undeviated beam, in turn, is shifted by a ring-shaped phase plate in the objective, so that the final phase difference between undeviated and diffracted light is about $\lambda / 2$. A phase difference of $\lambda / 2$, however, leads to destructive interference. Thus, sample-induced phase variations are translated into changes in brightness in the final image.

A final technique that was used in our experiments is high-resolution confocal microscopy. In particular, we used the technique for our staining experiments on glass. Confocal microscopy was performed using an IX81 FV1000 microscope (Olympus) equipped with $488 \mathrm{~nm}$ and $546 \mathrm{~nm}$ laser lines and a $60 \mathrm{x}$ oil objective with a numerical aperture of 1.42. Images were recorded with an Olympus FVS-PSU/IX2-UCB camera and scanning unit. The principle of confocal microscopy is similar to epifluo- 


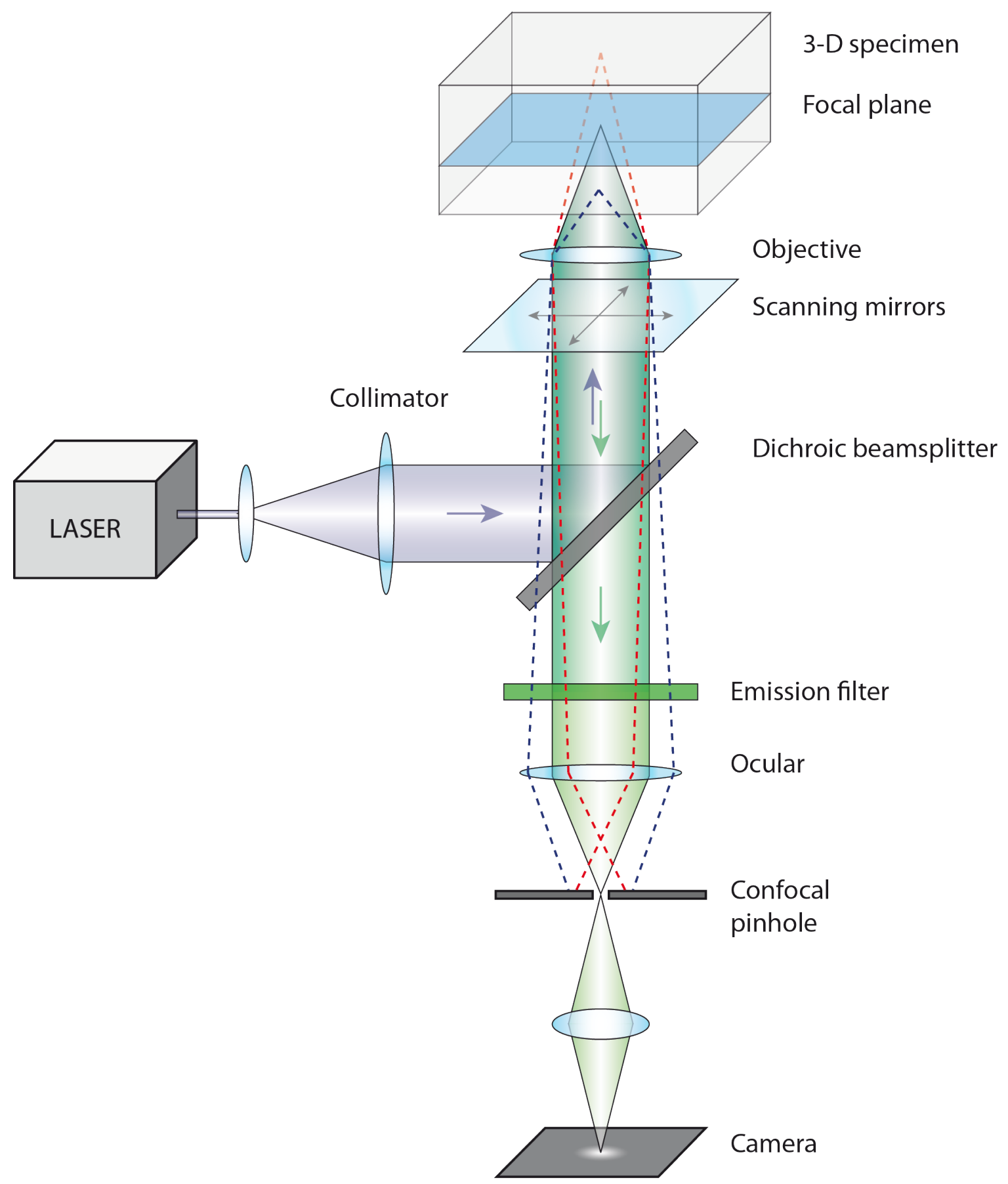

Figure III.7: Sketch of an inverted confocal scanning microscope: As for epifluorescence microscopy, the fluorescence signal of a previously labeled sample is collected during confocal microscopy. In a confocal setup, however, a pinhole aperture is introduced to reduce the size of the detected volume in a sample, thereby removing out-of-focus light. This detection volume is scanned over the sample to obtain a full image of the specimen under observation. [Figure by Jens Nolting] 
rescence microscopy described earlier in this section. The main difference between the two microscopy techniques is a confocal pinhole that is introduced into the light path of a confocal microscope to filter out any out-of-focus light emitted by the sample (figure III.7). Due to this pinhole, signals from the sample are detected only in a small region so that a scanning unit is introduced, which scans over the sample to reconstruct a final image of the sample. The technique efficiently removes blurring effects in fluorescence images, which are caused by out-of-focus light and hence images are sharper than those taken by conventional epifluorescence microscopy. 



\section{Data analysis}

As described in section III, we performed both TFM experiments as well as separate, fluorescence staining experiments on platelets that were activated on soft PAA substrates. Following a TFM recording, we obtained two sets of image stacks: one containing the displacement of fluorescence beads within the PAA, which is indicative of cell-induced substrate deformation, and the other showing the cellular behavior on top of the substrates. Subsequent data analysis steps for the TFM data sets involved extracting bead displacement maps and cellular contours from these two image stacks. The new information thus gained was used to calculate traction force fields. Traction force calculation was performed by the program LIBTRC (version 2.4.), which has kindly been provided by Micah Dembo from Boston University (MA, USA). For bead traction and cell contour tracing we discovered, however, that the standard techniques used in the community could not be applied to our data to any avail (section I.4). Instead, we developed our own MATLAB programs (version 7.9.0.529 (R2008b) with Image Processing Toolbox; The MathWorks Inc.) to cope with both the rapidly changing bead patterns and the huge amount of recorded data. In the following sections, we will give more detailed information on the different steps taken during TFM data analysis. In addition, we will describe smaller MATLAB programs written to assist in characterizing calculated traction force fields. Finally, we will refer to the second set of experiments performed on platelets and expand both on image preprocessing steps and on the criteria chosen for the selection of typical staining patterns.

\section{IV.1 Image Preprocessing}

At the beginning of TFM data analysis, all images were corrected for variations in illumination by a high pass filter using the freeware ImageJ. These variations in illumination were partly caused by the Xenon light source used in our microscope and partly they originated in diffraction patterns of out-of-focus platelets or in refraction at the liquid surface of the cell suspension. Next, we adjusted the contrast in the images and reduced noise by a selective mean filter (Sigma Filter Plus plugin for ImageJ by Michael Schmid and Tony Collins). We then converted the 16-bit images to 8-bit, as most of the MATLAB functions in subsequent steps required 8-bit color format. At this stage, we performed an initial drift correction for some TFM data sets (see section IV.2). Subsequently, we cropped images to include only single cells, and reduced image stack size to encompass only those time frames showing the cells after they had settled on the substrate $\left(I_{\text {Cells }}(t)\right.$ and $\left.I_{\text {Beads }}(t)\right)$. Here, we observed that the surface of the PAA substrates displayed some defects in the form of small fissures, which likely resulted from the removal of the overlying cover slip during PAA substrate preparation (figure IV.1. see also section III.2. . To prevent these fissures from having any influence on measure- 
ments, we took care in choosing only those cells for analysis that were sufficiently far away from any observable defect in the PAA. For later cell contour tracing, we generated for each single cell an additional image stack $I_{E n h}(t)$ with enhanced cell edges. This stack was obtained from the original phase contrast images of the cells by using a very selective high pass filter that removed any variations in intensity that had a wavelength larger than $4 \mathrm{px}$.

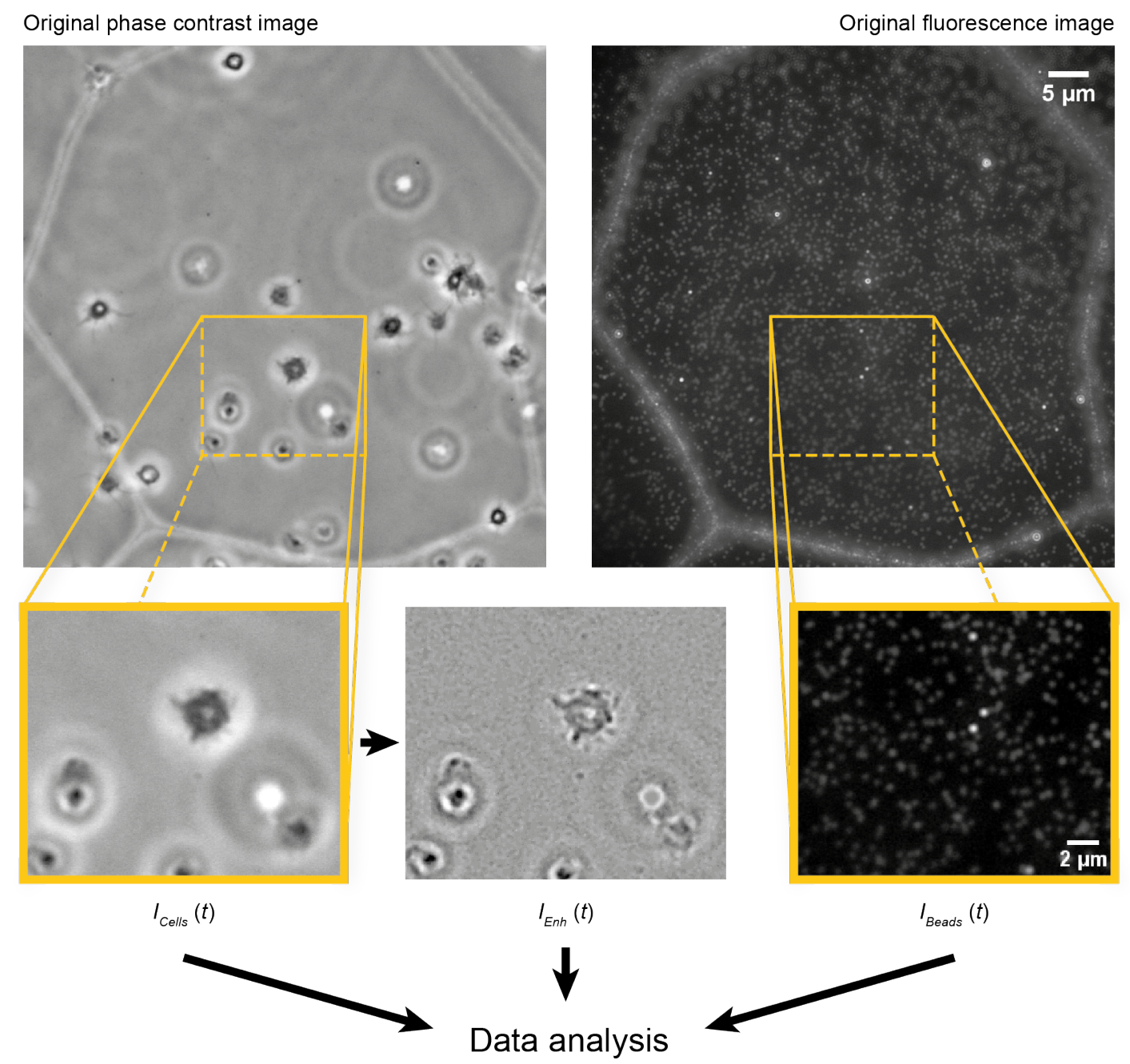

Figure IV.1: Example TFM data set at a time $t$ showing image preprocessing steps at the beginning of data analysis: Images taken of the platelets (top left) and the fluorescent beads in the underlying substrate (top right) during a TFM recording, were first corrected for noise and variations in illumination. They were then cropped to include only single activating platelets (bottom images). While selecting these single cells for further data analysis, we took care to choose only those cells which were far away from any visible defect in the substrates (fissures shown in top images). For these cells we created an additional image containing enhanced edges $\left(I_{E n h}(t)\right)$, which is later used to facilitate cell contour tracing (section IV.3). 


\section{IV.2 Bead Traction}

After having corrected the images, we tracked the beads in the recorded fluorescence images $\left(I_{\text {Beads }}(t)\right)$ with the help of a self-written MATLAB program. In fact, we tracked the beads twice. The first time, we used the result to estimate a drift correction for the recorded images. To that end, we plotted the detected bead displacements for each dimension $x_{i}(i=1,2)$ and each time $t$ in a histogram and identified the peak of the histogram as the drift in the images at time $[[1]$ (figure IV.2). Each image was subsequently corrected for the detected drift, and we repeated bead traction for the newly corrected images.

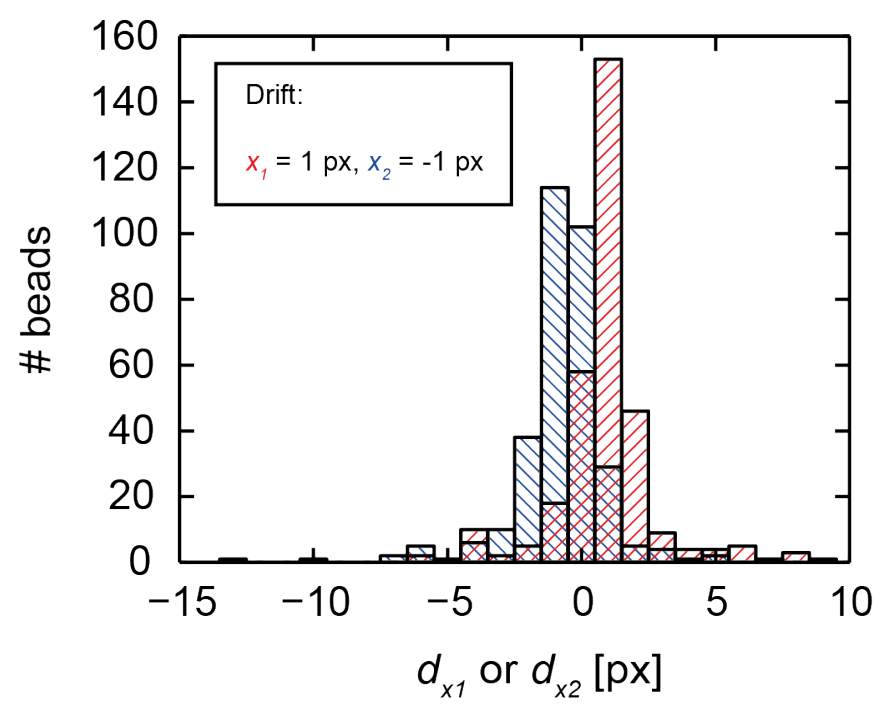

Figure IV.2: Drift was identified in our images according to the most abundant bead displacement vectors: The fluorescent beads in our TFM recordings were used to correct images for drift. Beads were tracked over time and the $x_{1}$ - and $x_{2}$-components of all bead displacements $\vec{d}$ at a time $t$ were collected in a histogram $\left(d_{x_{1}}\right.$ shown in red and $d_{x_{2}}$ shown in blue in the example above). The peak for each component then revealed the respective vector components of the drift in the images at time $t$.

During bead traction, we first reduced noise in all fluorescence images $I_{\text {Beads }}(t)$ by applying a Gaussian filter with variance $\sigma=1 \mathrm{px}$. The local intensity maxima in the first image of the stack $I_{\text {Beads }}(t)$ are then identified as beads. This image was taken to be the reference image ('null' image) for the subsequent calculation of bead displacements. By prompting a graphical user input, the MATLAB program gives the user the opportunity to manually correct the bead positions $\left(x_{1, i}^{0}, x_{2, i}^{0}\right)$ in the 'null' image. For all images following the 'null' image, local maxima were matched with beads according to the last known position of the beads and their last known direction of motion. In order to assign local maxima in a frame $t$ to beads known from frame $t-\Delta t$, we calculated the distance $r_{b}$ of each local maximum to the last known position

\footnotetext{
${ }^{1}$ Most beads during a TFM recording remained fixed in time and were undisturbed by cellular forces. Only beads in the vicinity of activating platelets were displaced due to cellular contraction. The beads that were unaffected by any cells made up the peak in the histogram of bead displacements, revealing image drift.
} 
a) Bead track, projected line of last known motion
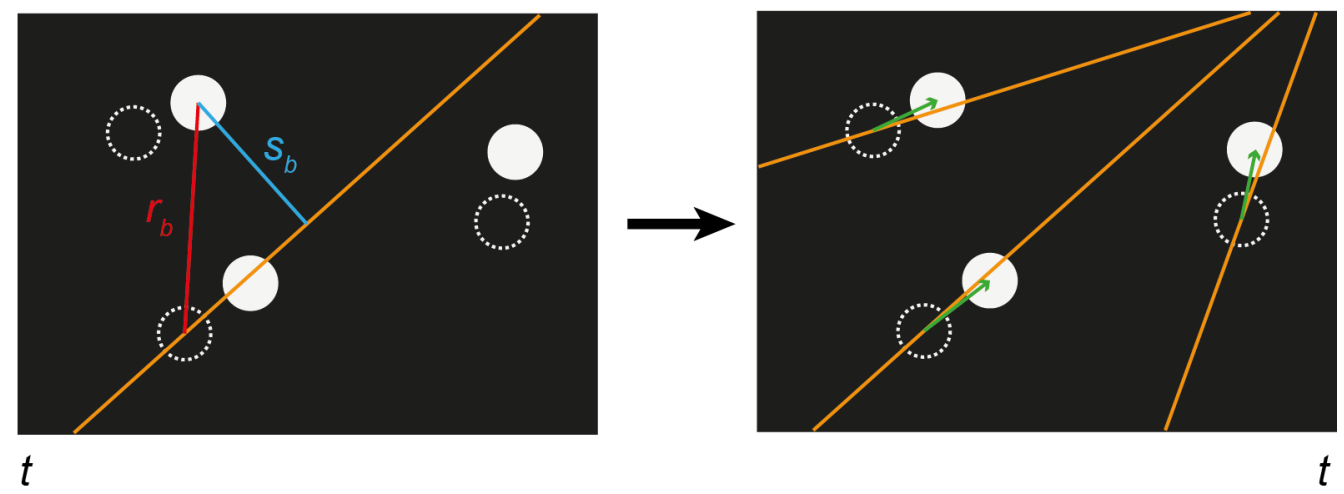

b)
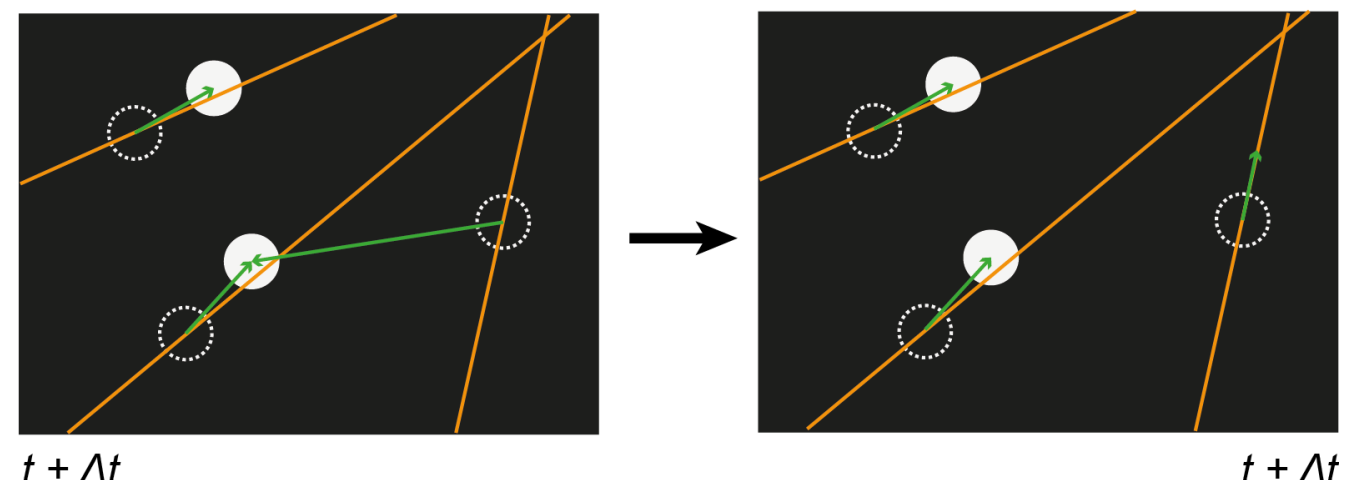

Figure IV.3: Sketch illustrating the particle tracking algorithm used for bead traction: a) During bead traction in the fluorescence images of a TFM recording, local intensity maxima in a time frame $t$ (full circles) were matched with the maxima in a frame $t-\Delta t$ (open, dotted circles) by minimizing $N_{c}=r_{b}+s_{b}$, where $r_{b}$ is the distance of any maximum at $t$ to a particular maximum $b$ at $t-\Delta t$. The parameter $s_{b}$, in turn, represents the distance of the maxima at $t$ to the projected line of motion for $b$ known from time frame $t-\Delta t$ (orange line). The best matches were used to calculate bead displacements (green arrows). b) Multiple matches during bead traction were solved by keeping the overall best match (smallest $N_{c}$ ) and reassigning the unmatched maximum at $t+\Delta t$ to its former position at $t$. 
$\left(x_{1, i}(t-\Delta t), x_{2, i}(t-\Delta t)\right)$ of every bead $i$. In addition, we looked at the smallest distance $s_{b}$ of each local maximum to the projected line of the last known bead motion (figure IV.3 a)), meaning the line defined by

$$
g_{i}:\left(\begin{array}{l}
x_{1} \\
x_{2}
\end{array}\right)=\left(\begin{array}{c}
x_{1, i}^{0} \\
x_{2, i}^{0}
\end{array}\right)+n \cdot\left(\begin{array}{l}
x_{1, i}(t-\Delta t)-x_{1, i}(t-2 \Delta t) \\
x_{2, i}(t-\Delta t)-x_{2, i}(t-2 \Delta t)
\end{array}\right)
$$

For the first few frames and whenever the bead position remained fixed over time, the parameter $s_{b}$ was set to zero. From $r_{b}$ and $s_{b}$, we calculated a third parameter $N_{c}$ for each bead $i$ :

$$
N_{c}(i)=r_{b}(i)+s c \cdot s_{b}(i)
$$

where $s c$ is a simple scaling factor. By minimizing equation IV.1, we finally identified the best match for any particular bead among the local maxima in a time frame $t$ (green arrows in figure IV.3 a)). This approach takes advantage of the fact that bead motion during platelet contraction is constant in its direction of motion. Hence, beads are preferably looked for along the direction of their last known motion. To reinforce the selective search, the parameter $s c$ can be increased. For all our data sets, we achieved good results with $s c=1$. It should be noted that equation IV.1 allows multiple beads to be matched with one local minimum in the time frame $t$. Whenever this occurred, we kept the match with the overall smallest $N_{c}$ and reassigned all other beads to their positions in the previous time frame $t-\Delta t$ (figure IV.3 b)).

Later on, the program LIBTRC calculating the traction forces required an input parameter Rerr for each bead and each time frame $t$ containing an error estimate for the bead traction. In accordance with the description of Rerr in the LIBTRC manual, we determined Rerr as follow: $[2]$;

$$
\operatorname{Rerr}(i, t)=\frac{\left|\overrightarrow{d_{i}^{s m}}(t)-\vec{d}_{i}(t)\right|}{\min \left(\left|\vec{d}_{i}^{s m}(t)\right|,\left|\vec{d}_{i}(t)\right|\right)}
$$

where $\vec{d}_{i}(t)=\left(\begin{array}{l}x_{1, i}(t)-x_{1, i}^{0} \\ x_{2, i}(t)-x_{2, i}^{0}\end{array}\right)$ is the displacement of any bead $i$, and $\vec{d}_{i}^{s m}(t)$ is the average displacement of a number $N$ of next neighboring beads (in our case $N=3$ or 5 , see also figure IV.4). That is, if $r_{i j}(t)$ at a time $t$ represents the distance between a bead $i$ and a bead $j$, and $M_{i, t}$ is the group of $N$ next neighbors to $i$ at $t$, then $\overrightarrow{d_{i}^{s m}}(t)$ is expressed as:

$$
\overrightarrow{d_{i}^{s} m}(t)=\frac{\sum_{j \neq i, j \in M_{i, t}} \frac{\vec{d}_{i}(t)}{r_{i j}}}{\sum_{j \neq i, j \in M_{i, t}} \frac{1}{r_{i j}}}
$$

${ }^{2}$ If $\left|\vec{d}_{i}^{s m}(t)\right| \approx 0$ or $\left|\vec{d}_{i}(t)\right| \approx 0$, we corrected the two terms as follows to prevent a division by zero in equation IV.2 $\left|\vec{d}_{i}^{s m}(t)\right|=0.5 / N$ and $\left|\vec{d}_{i}(t)\right|=0.5$. Here, $N$ is the number of next neighbors used in the calculation of $\vec{d}_{i}^{s m}(t)$ according to equation IV.3 (usually $N=3$ or 5 ). 

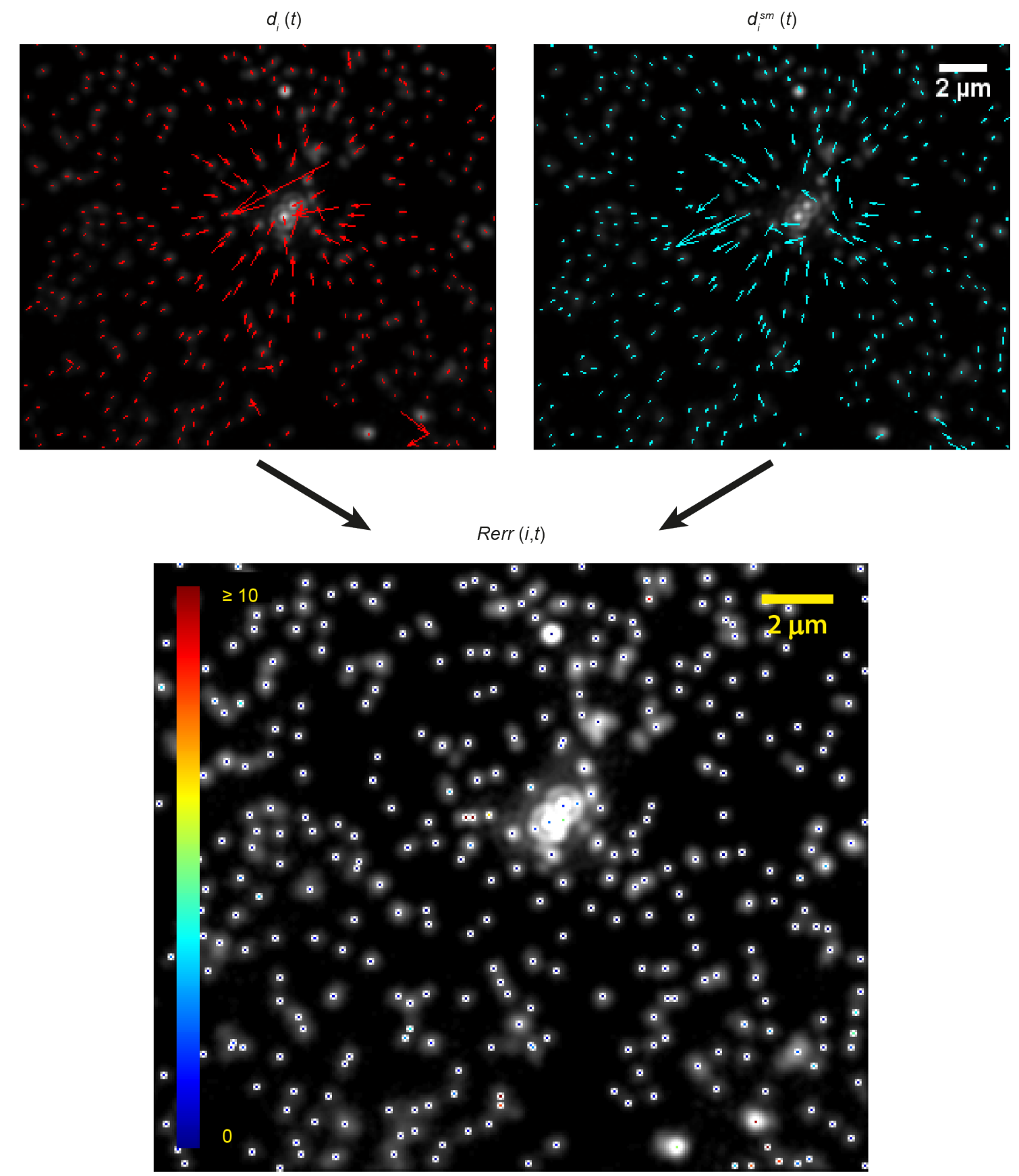

Figure IV.4: Errors for bead traction were estimated by calculating local variations in bead displacements: Traction force calculation during later steps in TFM data analysis required an estimate for bead traction errors. Such an estimate Rerr was provided by calculating the normalized difference between the displacement $\vec{d}_{i}(t)$ of a bead $i$ (red arrows in the bead image on the top left) and the average displacement $\vec{d}_{i}^{s m}(t)$ of its next neighbors (cyan arrows in the bead image on the top right). The result for $\operatorname{Rerr}(i, t)$ is shown as colored dots, which were overlaid with the bead image at the bottom of the figure. For better visibility of the dots, we encircled them in white and adjusted the contrast in the bead image. 

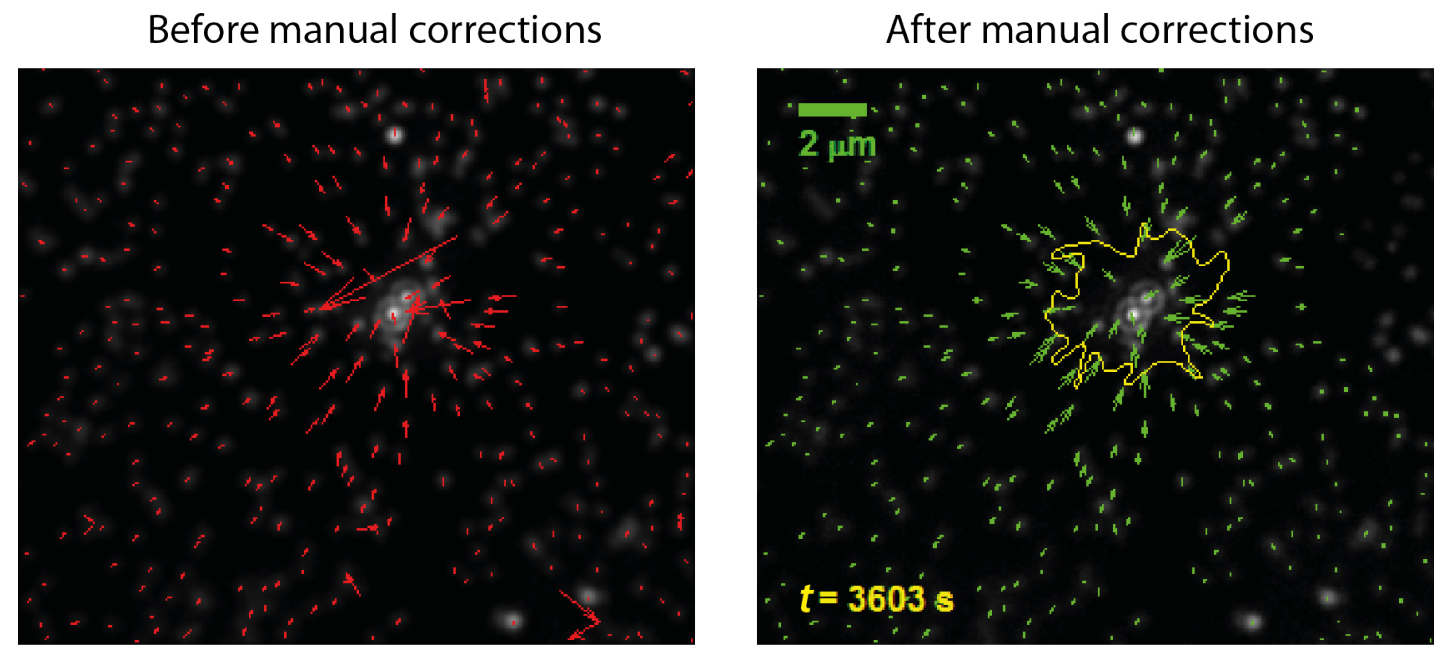

Figure IV.5: Bead displacements were manually corrected before traction force calculation: Example images showing fluorescence marker beads in a PAA substrate at a time $t$ during a TFM recording of platelet contraction. To visualize substrate deformation caused by platelet forces, images were overlaid with the bead displacements (red arrows) calculated for $t$. Following particle tracking, we corrected any bead mismatches manually (green arrows) and masked out regions, where bead displacements were affected by the influence of neighboring platelets. To visualize the position of the platelet under study, we have included the platelet's contour (see section IV.3) on the right (yellow line).

Following the automatic bead traction described above, manual corrections were performed with separate MATLAB programs to amend possible bead mismatching caused by noise in the bead images (figure IV.5). These programs enabled us to manually set the position of single beads at any particular time point $t$, after which automatic bead traction is resumed for these single beads for all remaining $[[3]$. Alternatively, if bead mismatching occurred for several $t$, the position of single beads could be set for a specified number of time frames 3 . Finally, beads could be masked out for the entire time track, if, for example, they were not only displaced by the cell under study but also by other cells in the vicinity. At the end of bead traction and all subsequent manual corrections, bead displacements were saved to individual text files for each $t$ with the following format:

\begin{tabular}{ccccc}
\hline$x_{1, i}^{0}$ & $x_{2, i}^{0}$ & $x_{1, i}(t)$ & $x_{2, i}(t)$ & $\operatorname{Rerr}$ \\
\hline$\ldots$ & $\ldots$ & $\ldots$ & $\ldots$ & $\ldots$ \\
\hline
\end{tabular}

\section{IV.3 Determination of Cellular Contours}

The next step during TFM data analysis consisted in detecting cellular contours in the phase contrast image stack, $I_{\text {Cells }}(t)$ (figure IV.6). Before contours were traced,

\footnotetext{
${ }^{3}$ Although only the bead displacements of selected beads were corrected here, Rerr was recalculated for all beads, as it changed not only for the beads under consideration, but also for all neighboring beads (equations IV.2 and IV.3.
} 
however, our MATLAB program applied a combination of a Gaussian, a median and a Wiener filter to each phase contrast image in the stack to minimize noise. While the Gaussian filter reduced noise by smoothing the gray values in the images, salt and pepper noise was removed by the median filter, and high frequency noise was eliminated by the low pass Wiener filter. Noise removal was furthermore extended to the image stack $I_{E n h}(t)$, obtained from $I_{\text {Cells }}(t)$ by a very selective high pass filter (section IV.1). Following noise removal, the initial position of a cell of interest was specified by graphical user input in the first image of $I_{\text {Cells }}(t)$, in order to be able to track the cell while detecting its contour. The images $I_{C e l l s}(t)$ were then binarized $\left(B I_{\text {Cells }}(t)\right)$ by using a threshold $T h r$ for the gray values in $I_{\text {Cells }}(t)$. This threshold $T h r$ was set to the background in each image. That is, the median for every vertical line in an image was calculated and the smallest median among the group was chosen to be Thr. Next, a Laplacian filter was used on $I_{E n h}(t)$, meaning that for each image we calculated $\vec{\nabla}^{2} \cdot I_{E n h}\left(\vec{x}_{1}, \vec{x}_{2}, t\right)$. The result was used to create another binarized image $B I_{E n h}(t)$ for $\vec{\nabla}^{2} \cdot I_{E n h}\left(\vec{x}_{1}, \vec{x}_{2}, t\right)>0$. The binary images from both $I_{\text {Cells }}(t)$ and $I_{E n h}(t)$ were then combined and all objects smaller than a user-specified area threshold were filtered out. In the resulting binarized images $\left(B I_{\text {unlinked }}(t)\right)$, the cell of interest was identified among all objects in $B I_{\text {unlinked }}(t)$ according to its last known position in the previous time frame $t-\Delta t$. At this point, however, most filopodia of the identified cell were as yet unconnected to the cell body in $B I_{\text {unlinked }}(t)$. To put the cell together, we first dilated the cell body in image $B I_{\text {Cells }}(t)$ and made out unconnected parts of the cell as those objects in $B I_{\text {unlinked }}(t)$ which had an overlap with the dilated image $B I_{\text {Cells }}(t)$. Having identified the objects to be connected, we performed a morphological closure operation on them (MATLAB function imclose) and filled up any holes that were present in the final binarized images of the cell $B I_{\text {linked }}(t)$. For these final images, we traced the contour of the cell in clockwise direction and saved the coordinates of all points on the contour to a separate text file for each $t$. In addition, we saved the time track of the cell area's center $\left(x_{1, c}(t), x_{2, c}(t)\right)$ to yet another text file, in which we also included the temporal evolution of the cell area $A(t)$.

The automatic detection of cellular contours that we have just described, though satisfactory, is error-prone. In particular, when a second cell came into close proximity of the cell of interest, our MATLAB program sometimes failed to recognize the two cells as separate entities. To intercept these special cases, our program kept track of the cell area $A$. Whenever it encountered a large difference in $A$ between consecutive time frames, it prompted a user input that made it possible for the cell contours to be drawn manually. Other errors introduced by our program could not be corrected as easily. For example, we noticed that for each $t$ there were still some filopodia that were not recognized by our program (see also figure IV.6). Here, the large amount of recorded TFM data prevented us from performing manual corrections for all $t$. We did write a MATLAB program to be able to manually correct at least those contours for which automatic detection failed most noticeably $\left[{ }^{[4]}\right.$. For the bulk of our data, however, we accepted the results from our automatic contour detection and concentrated instead on estimating how the errors introduced by our program influenced our final results on platelet contraction. As shown in section VI.1 this influence is, in fact, rather small.

${ }^{4}$ Following such manual corrections, we updated the text file containing $x_{1, c}(t), x_{2, c}(t)$ and $A(t)$. 


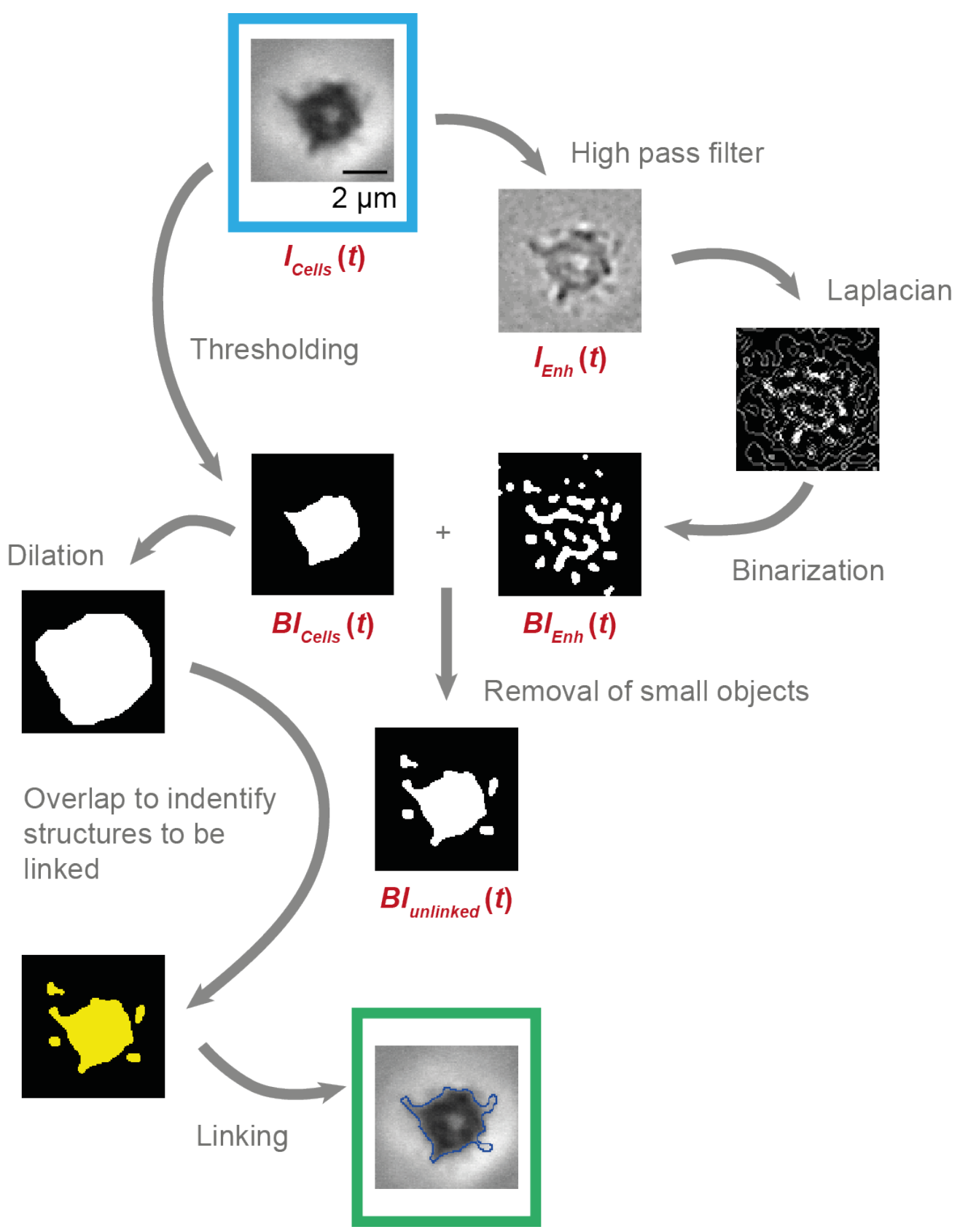

Figure IV.6: Sketch illustrating the steps involved in cell contour tracing: Cell contour tracing for a TFM data set was performed by applying a gray value threshold to every phase contrast image of a cell (example shown is enclosed by blue rectangle), and combining the result with a binarized image of cellular edges. The latter was, in turn, obtained by using both a high pass filter and a Laplacian filter on each cellular phase contrast image, which was followed by a binarization step. From the combined binarized images of the cell, small objects were then removed and cellular fragments were linked together by applying a morphological closure operation. To identify cellular fragments, the cell body was dilated and all objects overlapping with it were taken to be part of the cell. In the final binarized image of the cell we traced the contour, which is shown above as a blue line in the final image of the cell (enclosed by a green rectangle). 


\section{IV.4 Calculation of Traction Force Fields}

Using as input parameters the bead displacements and cellular contours from the previous sections IV.2 and IV.3, we finally calculated the traction forces for all time points $t$ with the help of the program LIBTRC. Traction force calculation by LIBTRC was performed for the nodes in a mesh $M$ that was spanned over the cell area at any $t$. Initially, this mesh was chosen to be a coarse-grained, rectangular grid, which was progressively refined in a series of iterations (LIBTRC command hacscript). The number of iterations, and hence the final mesh size, was influenced by a user-specified parameter $m r$, termed mesh resolution index. In our case, we set $m r=4$, which enabled us to minimize computation time while still calculating traction force fields at maximal resolution (section IV.6). To conclude mesh generation, the rectangular shape of some segments of the grid was dispensed with and single mesh nodes were moved so that the overall appearance of $M$ resembled more closely the cell shape (figure IV.7). For the nodes $\vec{x}_{n}$ in the final mesh $M$, traction forces $\vec{T}\left(\vec{x}_{n}\right)$ were then calculated (LIBTRC command fitscript) by using a regularization approach to the following equation (see also section I.4):

$$
d\left(\vec{x}_{b}\right)=\sum_{n} \underline{\underline{G}}\left(\vec{x}_{b}-\vec{x}_{n}\right) \vec{T}\left(\vec{x}_{n}\right) \mathrm{d} A_{n}
$$

where $d\left(\vec{x}_{b}\right)$ represents the displacement of any bead $b, \mathrm{~d} A_{n}$ is the area of a mesh segment and $\underline{\underline{G}}$ is Boussinesq's solution ${ }^{[5]}$ (equation II.39 in section II.1.2. In the following, we will explain in more detail how LIBTRC calculated $\vec{T}$ from equation IV.4. To distinguish our measured bead displacements from those calculated for any solution of $\vec{T}$, we will subsequently denote the former $\vec{d}_{e x}\left(\vec{x}_{b}\right)$ and the latter $\vec{d}_{t h}\left(\vec{x}_{b}\right)$.

To obtain a realistic solution for the traction force field $\vec{T}$, the LIBTRC program first considered two extreme scenarios: one, where traction forces were zero, and another, where traction forces took on the simplest conceivable form given measured bead displacements ("best fit"). The "best fit", in this case, refers to the particular solution $\vec{T}_{B F T}$ that minimizes

$$
\chi^{2}=\sum_{b=1}^{N_{b}}\left(\vec{d}_{e x}\left(\vec{x}_{b}\right)-\vec{d}_{t h}\left(\vec{x}_{b}\right)\right)^{2}
$$

where $N_{b}$ is the number of detected beads. The minimal $\chi^{2}$ for $\vec{T}_{B F T}$ is termed CHIBFT in the LIBTRC program, while the value for $\chi^{2}$ for $\vec{T}_{N U L}=0$ was set to:

$$
\mathrm{CHINUL}=\sum_{b=1}^{N_{b}}\left(\vec{d}_{e x}\left(\vec{x}_{b}\right)-\left\langle\vec{d}_{e x}\right\rangle\right)^{2}
$$

These two values for $\chi^{2}$ were taken to be lower and upper limits for any realistic solution, $\vec{T}$. LIBTRC next devised what $\chi^{2}$ should look like based on experimental

\footnotetext{
${ }^{5}$ LIBTRC actually employed a modification of Boussinesq's solution, which included correction terms for the fact that the PAA substrates used for TFM experiments had a finite thickness. Unfortunately, the LIBTRC manual gives no further details about the corrections made, as these have not been published yet.
} 


\begin{tabular}{|c|c|c|}
\hline Parameter & Short description & Value \\
\hline YMODU & Young's modulus of PAA substrate & $\sim 4 \mathrm{kPa}$ \\
\hline POSRA & Poisson ratio of PAA & 0.3 \\
\hline TFILM & thickness of substrate & $\begin{array}{l}\text { set individually for } \\
\text { each TFM data set }\end{array}$ \\
\hline BDIAM & diameter of beads in PAA & $0.1 \mu \mathrm{m}$ \\
\hline ZBAVE & $\begin{array}{l}\text { distance of bead layer from top } \\
\text { of substrate }\end{array}$ & $-0.6 \mu \mathrm{m}^{[2]}$ \\
\hline $\begin{array}{l}\text { NPIXX } \\
\text { and NPIXY }\end{array}$ & $\begin{array}{l}\text { size of images used for data analysis } \\
\text { (pixel units) }\end{array}$ & $\begin{array}{l}\text { set individually for } \\
\text { each TFM data set }\end{array}$ \\
\hline NBITS & color format in images & 8 bit \\
\hline ZDEEP & depth of the optical section & $5 \mu \mathrm{m}$ \\
\hline $\begin{array}{l}\text { MPPX } \\
\text { and MPPY }\end{array}$ & size of an image pixel & $64.5 \mathrm{~nm}$ \\
\hline REGMTH & $\begin{array}{l}\text { parameter decisive of the regulari- } \\
\text { zation method used during traction } \\
\text { force calculation (equation IV.9P }\end{array}$ & 0 \\
\hline CONMTH & $\begin{array}{l}\text { parameter decisive of force and } \\
\text { torque constraints included during } \\
\text { traction force calculation }\end{array}$ & 0 (no constraints) \\
\hline SOLMTH & $\begin{array}{l}\text { parameter influencing convergence } \\
\text { criteria for iterations during traction } \\
\text { force calculation }\end{array}$ & $\begin{array}{l}0 \text { (default conver- } \\
\text { gence criteria) }\end{array}$ \\
\hline BASMTH & $\begin{array}{l}\text { parameter controlling how LIBTRC } \\
\text { reads SIGDEX (more details on the } \\
\text { choices for BASMTH can be found } \\
\text { in the LIBTRC manual) }\end{array}$ & 3 \\
\hline SIGDEX & $\begin{array}{l}\text { estimate of the standard deviation } \\
\text { of the error in a single component } \\
\text { of the bead displacements }\end{array}$ & $0^{[4]}$ \\
\hline SIGFAC & $\begin{array}{l}\text { scaling of a correction term for } \\
\text { SIGDEX used during traction force } \\
\text { calculation (equation IV.7) }\end{array}$ & $0-1.5$ \\
\hline
\end{tabular}

1 TFILM was set according to measurements of the substrate thickness (section III.4).

2 A LIBTRC default value was used here, as ZBAVE could not be easily measured due to limitations in optical resolution.

${ }^{3}$ Information on the depth of the optical section was not readily available so that again a LIBTRC default value was used.

${ }^{4}$ For our choice of BASMTH, LIBTRC ignored the value set here and instead estimated SIGDEX from the bead displacements at the periphery of our images.

Table IV.1: LIBTRC parameters chosen for our TFM data analysis 
errors in $\vec{d}_{e x}\left(\vec{x}_{b}\right)$ :

$$
\mathrm{CHICRT}=2 N_{b}[\mathrm{SIGDEX}]^{2}+\mathrm{SIGFAC} \cdot \mathrm{CHINULSIG}
$$

where SIGDEX is the standard deviation of the error for a single component of $\vec{d}_{e x}\left(\vec{x}_{b}\right)$, SIGFAC is a user-specified scaling factor and CHINULSIG is an estimate for the standard deviation of CHINUL. That is, CHINULSIG specifies the variations that would be measured in CHINUL if one were to repeat the bead detection and bead traction several times for the same experiment. The second term in equation IV.7 is therefore a correction term introduced to account for any uncertainty in the estimation of SIGDEX. According to the LIBTRC manual, statistical arguments can be used to find the following expression for CHINULSIG:

$$
\text { CHINULSIG }=\sqrt{\sum_{b=1}^{N_{b}}\left[\left(\vec{d}_{e x}\left(\vec{x}_{b}\right)-\left\langle\vec{d}_{e x}\right\rangle\right)^{4}\right]-\frac{\mathrm{CHINUL}^{2}}{2 N_{b}}}
$$

For our particular choice of LIBTRC parameters (see table IV.1), SIGDEX was furthermore estimated automatically from the bead displacements at the periphery of our images. In these regions, the so called far field, cellular influences on bead displacements are generally assumed to be non-existent, so that any variability in bead displacements reflects experimental errors only. To promote a good estimate for SIGDEX, we ensured that images were always large enough to include several beads in the far field.

Next, LIBTRC compared the value obtained for CHICRT with CHINUL and CHIBFT. If CHICRT was found to be larger or equal to CHINUL, then $\vec{T}_{N U L}(\vec{x})=0$ was taken to be the final solution for the traction forces. If CHICRT was found instead to be smaller than CHIBFT, LIBTRC issued a warning, but still chose $\vec{T}_{B F T}(\vec{x})$ to be the final solution for the traction forces $\left[\left[{ }^{[6]}\right.\right.$ For all other cases, LIBTRC searched for a final solution to $\vec{T}(\vec{x})$ by minimizing the following function:

$$
\text { BAYFUN }=\sum_{p}\left[\left\langle\left\|\vec{T}\left(\vec{x}_{p}\right)\right\|^{2}\right\rangle-\text { REGMTH }\left\|\left\langle\vec{T}\left(\vec{x}_{p}\right)\right\rangle\right\|^{2}\right]
$$

where REGMTH is a user-specified scaling factor and where

$$
\left\langle\left\|\vec{T}\left(\vec{x}_{p}\right)\right\|^{2}\right\rangle:=0.25 \sum_{i=1}^{4}\left\|\vec{T}\left(\vec{x}_{p i}\right)\right\|^{2} \text { and }\left\|\left\langle\vec{T}_{p}\right\rangle\right\|^{2}:=\left\|0.25 \sum_{i=1}^{4} \vec{T}_{p i}\right\|^{2}
$$

for any segment $p$ of the mesh $M$ mentioned at the beginning of this section. Here, $\|\vec{a}\|=\sqrt{a_{x 1}^{2}+a_{x 2}^{2}}$ represents the Euclidean norm for any vector $\vec{a}$, and the vectors $\vec{T}\left(\vec{x}_{p i}\right)$ are the traction forces at the 4 corners of the mesh segment $p$.

While minimizing BAYFUN, LIBTRC enforced the additional constraint $\chi^{2} \leq$ CHICRT. Optionally, further constraints on calculated forces or torques could be imposed by the user, which we, however, refrained from doing so as not to introduce

6 This particular case actually never occurred during our data analysis. 
any additional bias to our results. We could not fully prevent our results from being biased, as the regularization (equation IV.9) itself, used for solving equation IV.4, influenced traction force field morphology to some degree. For our particular choice of REGMTH, for example, regularization favored simple solutions for $\vec{T}$ with a minimal amount of contributions to the traction force field.
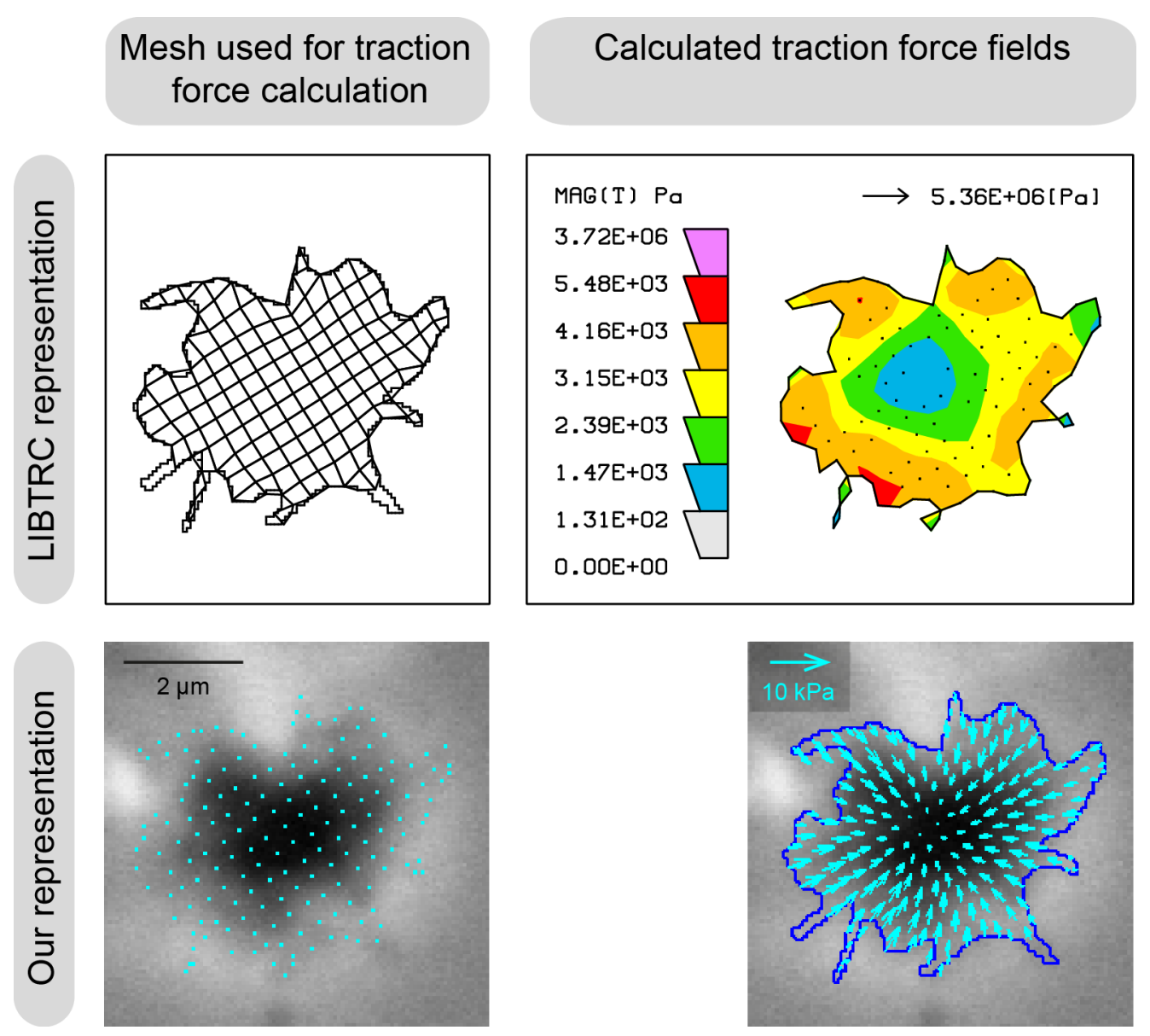

Figure IV.7: Traction force calculation was performed for the nodes in a mesh $M$ that was spanned over the cell area: The mesh used by the program LIBTRC to calculate traction forces for a platelet is shown on the left hand side. The top left representation is an excerpt from the output files saved by LIBTRC. To provide us with more flexibility, however, we read out mesh node positions from the LIBTRC output and plotted them ourselves as cyan dots over phase contrast images of the cells (bottom left). On the right hand side, in turn, is depicted the result of traction force field calculation by LIBTRC. As before, the top right is taken from LIBTRC output files, where the magnitude of traction forces is shown in color and the traction force vectors are represented by arrows. Due to the way LIBTRC normalizes arrows in a TFM data set, traction forces vectors only show as dots in the example above. For this reason we created again our own representation of the traction force fields and set it on top of cellular images (bottom right).

LIBTRC yielded not only a solution for the traction forces $\vec{T}\left(\vec{x}_{n}\right)$ at the nodes $\vec{x}_{n}$ of the mesh $M$ (figure IV.7). It furthermore extrapolated $\vec{T}$ to single pixels in our TFM images and saved this as an additional output $\left(\vec{T}_{p x}\right)$. Also, it calculated a few 
characteristics for $\vec{T}$ such as the average traction force

$$
\langle\|\vec{T}\|\rangle_{A}=\frac{1}{A} \int_{A}\|\vec{T}(\vec{x})\| \mathrm{d} A
$$

or the total force

$$
F_{t o t}=\int_{A}\|\vec{T}(\vec{x})\| \mathrm{d} A
$$

or the matrix $M_{i j}$ of traction moments

$$
\begin{aligned}
M_{i j}= & \frac{1}{2} \int_{A}\left(x_{i}-x_{c i}\right)\left(T_{j}-<T_{j}>_{A}\right) \mathrm{d} A \\
& +\frac{1}{2} \int_{A}\left(x_{j}-x_{c j}\right)\left(T_{i}-<T_{i}>_{A}\right) \mathrm{d} A
\end{aligned}
$$

where $\left(x_{c 1}, x_{c 2}\right)$ is the center of the cell area $A$. In particular, LIBTRC determined the Eigen values, $D_{+}$and $D_{-}$, for $M_{i j}$, which corresponded to the force dipole moments of the major and minor axis of contraction/dilation in $\vec{T}$ :

$$
D_{ \pm}=0.5\left[M_{11}+M_{22} \pm \sqrt{\left(M_{11}-M_{22}\right)^{2}+4 M_{12}^{2}}\right]
$$

\section{IV.5 Determination of the Time Point of Adhesion}

The output provided by LIBTRC (section IV.4) had, at this stage, no clearly defined time point $t=0$. To set a starting point to our temporal record, we returned to our results from the detection of cellular contours (section IV.3) and looked at the time track of the center $P_{C}$ of the area $A$ identified for a single cell. For precisions sake, we should actually refer to $A$ as a projected cell area, seeing that we already traced cellular contours before the cell had properly adhered to the substrate. For the time points before cellular adhesion, contours were traced for the projections of the moving cell onto the plane of the substrate's surface. Plotting the distance traveled by $P_{C}$ over the time frame number $n_{t}$, we obtained a saturation curve (figure IV.8). The plateau in this curve corresponded to the state of the cell after adhesion to the substrate. By fitting an empirical function

$$
f\left(n_{t}\right)=a\left(1-e^{-b n_{t}}\right)
$$

to the curve, we determined the point of cellular adhesion to be the $n_{t_{0}}$, for which:

$$
a-f\left(n_{t_{0}}\right)=\sqrt{\frac{1}{N} \sum_{n_{t}=1}^{N}\left(f_{r}\left(n_{t}\right)\right)^{2}}
$$

where $f_{r}\left(n_{t}\right)$ represents the fit residues rounded up to 0.5 pixels accuracy. The time point $t_{0}$ corresponding to $n_{t_{0}}$ was then set as the starting point of platelet activation $(t=0)$. 


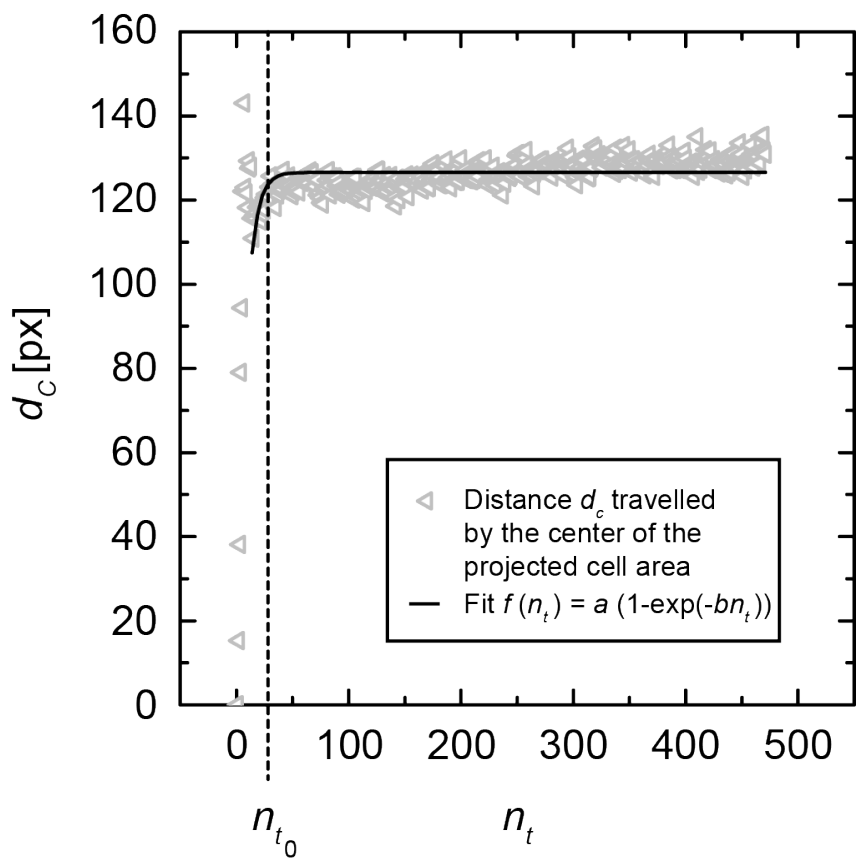

Figure IV.8: The time point of cellular adhesion to the substrate was identified as the moment, at which the cell stopped moving: The center of the cell area seen in phase contrast images during a TFM recording was tracked over time and the distance $d_{C}$ traveled was plotted against the time frame number $n_{t}$. The resulting graph shows a saturation curve. By fitting an appropriate function $f\left(n_{t}\right)=a\left(1-e^{-b n_{t}}\right)$ to it, we determined the point of cellular adhesion to be at that $n_{t_{0}}$, for which the difference between the saturation value $a$ and the fit function $f\left(n_{t_{0}}\right)$ was on the order of the fit residues. The time point $t_{0}$, corresponding to $n_{t_{0}}$, was subsequently marked as the starting point of platelet activation $(t=0 s)$.

\section{IV.6 Estimation of the Traction Force Fields' Spatial Resolution}

Calculated traction force fields (section IV.4) have a spatial resolution, which is determined by how accurately substrate deformations are detected via fluorescent marker beads in a TFM recording. The crucial factor influencing our spatial resolution is therefore the bead density at the surface of the substrates. To estimate a typical bead density for our experiments, we wrote a MATLAB script that connects all beads in an image of the undeformed substrate into a network with minimal and smallest connections (figure IV.9]. For each substrate used, we determined the average distance of these connections. The results were again averaged for all substrates, yielding a typical distance between beads of $\sim 0.8 \mu \mathrm{m}$. To see how this translates into a spatial resolution of our traction force fields, we picked a TFM data set with typical inter-bead distances and evaluated traction forces with an ever decreasing size of the mesh $M$, which is used to calculate traction forces (section IV.4). The results are shown in figure IV.10. For each mesh, we used again our program to extract a typical distance between mesh 
nodes, which was taken to be the mesh size $a_{M}\left[{ }^{[7]}\right.$ (see also figure IV.11 in section IV.7). We find that for a mesh size smaller than $0.2-0.3 \mu \mathrm{m}$ calculated traction force fields differ only little. Hence, we estimate the spatial resolution in our traction force fields to be $0.2-0.3 \mu \mathrm{m}$. This is also the range of sizes chosen for $M$ during traction force calculation for any of our TFM data sets. By choosing $M$ to be thus, we minimized computation time without loss of information.

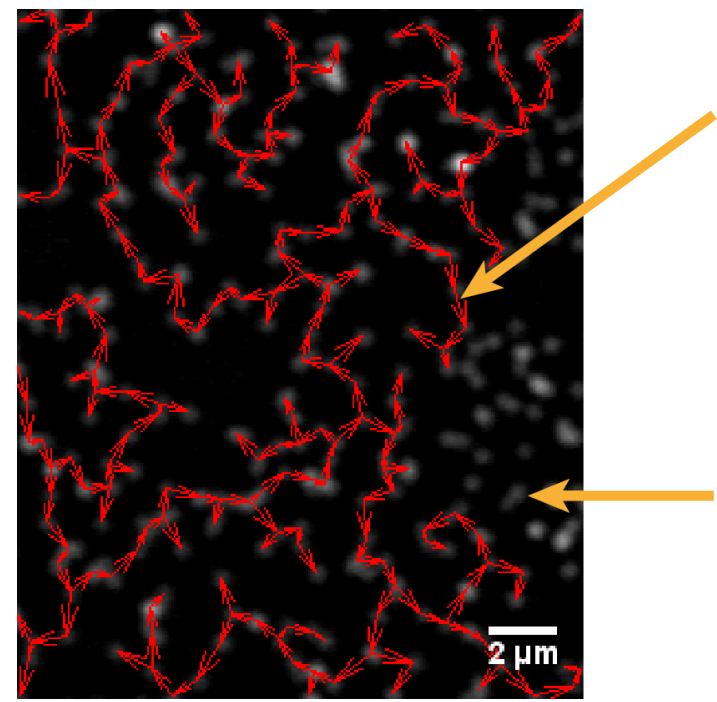

Minimal
connections
between
beads
Beads were
masked out
during TFM
data analysis
as they feel the
influence of
neighboring
cells

Figure IV.9: Bead spacing at the surface of our substrates was determined to be $\sim 0.8 \boldsymbol{\mu m}$ : To evaluate the bead density at the surface of the substrates used for TFM experiments, we first connected all beads into a network of minimal connections (red arrows). Some beads in the example shown above were not included into the network due to the fact that they had previously been masked out during TFM data analysis to prevent neighboring cells from taking an influence on TFM results (section IV.2). In the network that we obtained for the beads, we then averaged the distances of all connections. Finally, we averaged the result for all substrates used so that our final estimate for typical inter-bead distances in our experiments yielded $\sim 0.8 \mu \mathrm{m}$.

\section{IV.7 Preparation of Traction Force Field Characterization}

\section{IV.7.1 Calculating the Divergence of Traction Force Fields}

The traction force fields $\vec{T}(\vec{x})$ calculated in section IV.4 will form the basis of our analysis on platelet contractile forces in chapter $\mathrm{V}$. In this chapter, $\vec{T}(\vec{x})$ will be characterized by, for example, determining its divergence: $\vec{\nabla} \cdot \vec{T}(\vec{x})$. To prepare this step of data analysis, we removed noisy variations in $\vec{T}(\vec{x})$ at the current stage of data processing. In particular, we looked at the LIBTRC output, where $\vec{T}$ was extrapolated to

\footnotetext{
${ }^{7}$ We followed this procedure to determine $a_{M}$ in order to account for the fact that the size of mesh segments in $M$ was not constant (see section IV.4.
} 


\section{Mesh size for traction force field calculation:}

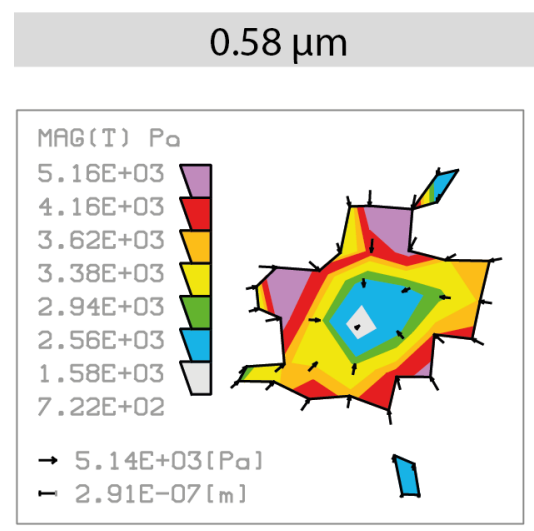

$0.46 \mu \mathrm{m}$

$0.28 \mu \mathrm{m}$
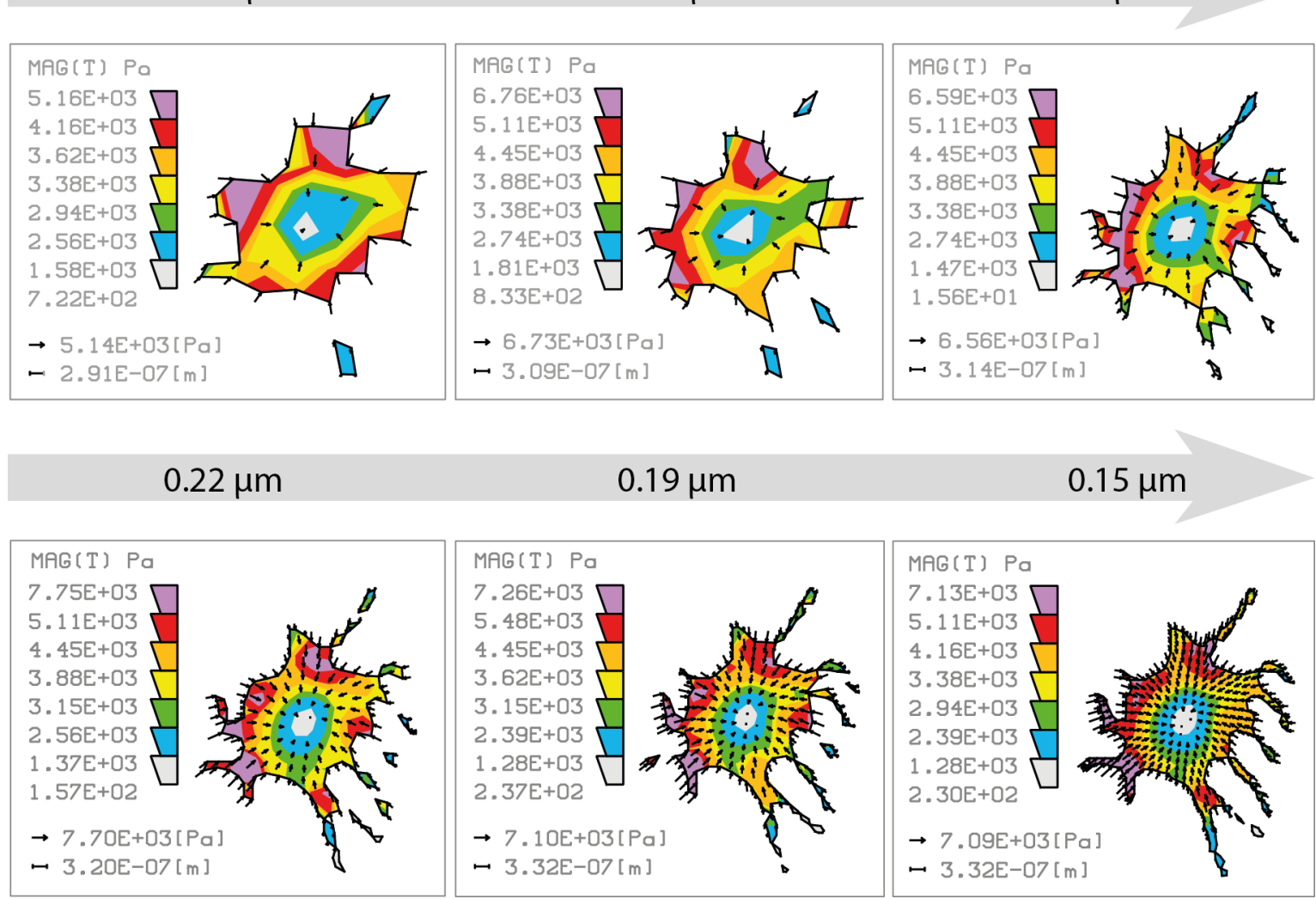

• $3.14 \mathrm{E}-07[\mathrm{~m}]$

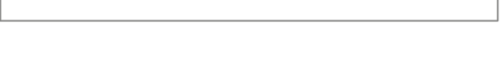

\section{$0.22 \mu \mathrm{m} \quad 0.19 \mu \mathrm{m}$}

$0.15 \mu \mathrm{m}$

Figure IV.10: Calculated traction force fields for platelets have a spatial resolution of $0.2-0.3 \mu \mathrm{m}$ : For a typical TFM data set, we repeated traction force calculation at some $t$ for the nodes in an ever finer mesh (section IV.4). The results for each mesh are shown as color-coded maps of traction force magnitudes, into which the traction force vectors have been drawn. As demonstrated by these maps, the force fields do not differ much for mesh sizes smaller than $\sim 0.2-0.3 \mu \mathrm{m}$, so that this range of sizes marks our spatial resolution.

single pixels $\left(\vec{T}_{p x}\right)$. This LIBTRC output $\vec{T}_{p x}$ was then smoothed $\left(\vec{T}_{p x}^{s m}\right)$ by averaging $\vec{T}_{p x}$ at each pixel position over a number $n_{N N}=\pi\left(2 a_{M}\right)^{2}$ of next neighboring pixels. Here, the parameter $a_{M}$ refers to the typical size, in pixel units, of the mesh $M$ that LIBTRC initially used to calculate $\vec{T}(\vec{x})$ (section IV.4). The parameter $a_{M}$ was, in turn, obtained by interlinking the mesh nodes in $M$ with a set of minimal and smallest connections, and averaging the distances of these connections (figure IV.11, see also section IV.6. The divergence of $\vec{T}(\vec{x})$ calculated in later sections, will then correspond to $\vec{\nabla} \cdot \vec{T}(\vec{x}, t) \approx \vec{\nabla} \cdot \vec{T}_{p x}^{s m}(\vec{x}, t)$ for each time frame $t$.

\section{IV.7.2 Extraction of a Typical Cell Radius}

Later, in chapter V, we will furthermore need an estimate for a typical cell radius $R$. To obtain $R$ we went back to the movies of binarized images $B I_{\text {linked }}(t)$ of single cells which were calculated from a TFM recording during cell contour tracing (section IV.3). 


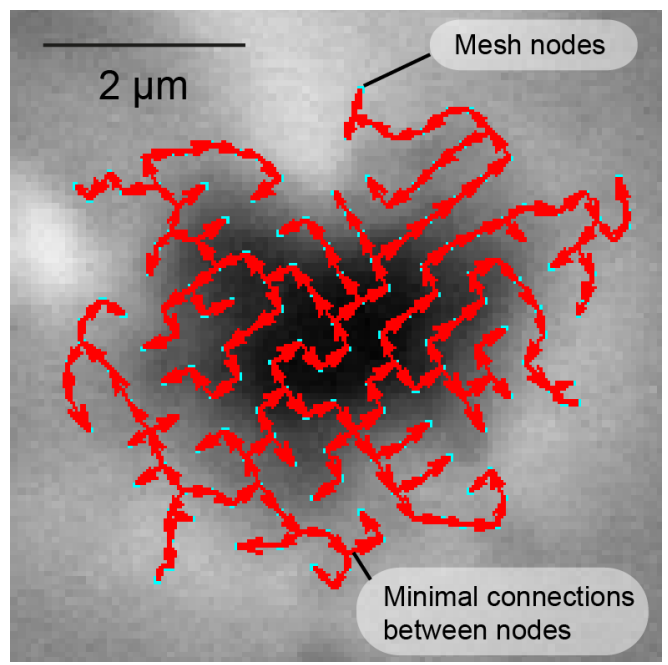

Figure IV.11: The typical size of the mesh $M$ used to calculate traction force fields was determined by linking all mesh nodes into a network of minimal connections: To smooth traction force fields during TFM data analysis, we needed to determine the typical size $a_{M}$ of the mesh $M$, for the nodes of which (cyan dots) traction force calculation had been performed (section IV.4). As the mesh was not a regular one, we decided to link mesh nodes into a network of smallest connections (red arrows) and calculated an average distance for these links. This average distance was then chosen to be $a_{M}$.
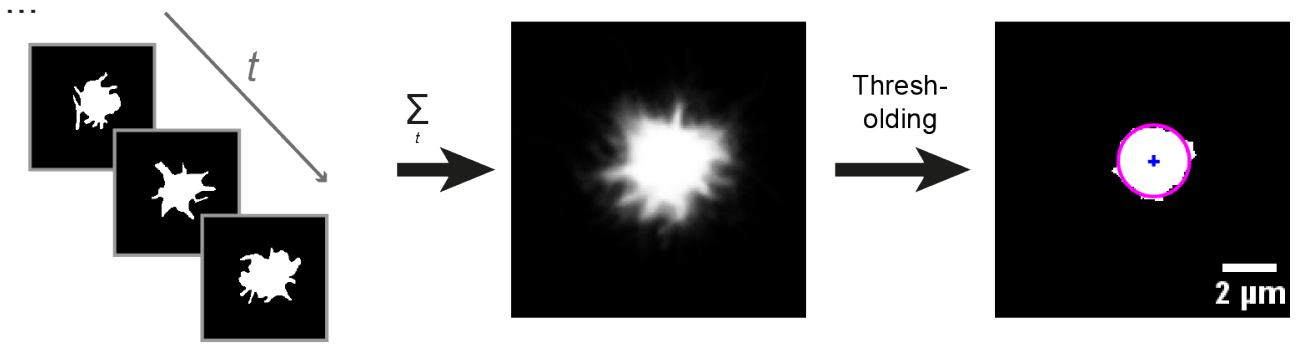

Figure IV.12: Sketch of the procedure used to determine a typical cell radius $\boldsymbol{R}$ : From cell contour tracing in a TFM data set, we obtained binarized images of platelets during activation on PAA substrates (section IV.3). These were then accumulated for all times $t$ to create an image overlay. By applying a gray value threshold to the overlay, we obtained a binarized image of an average cell area. In this image, we calculated the average distance between each point on the contour and the center of the cell area (blue cross). This average distance was finally used as a measure for a typical cell radius $R$ (a circle with radius $R$ is shown in magenta). 
We first generated an overlay $\overline{B I}_{\text {linked }}$ of $B I(t)_{\text {linked }}$ for all time points $t$, in which the single platelets were fully spread $[8]$. We then performed thresholding on $\overline{B I}_{\text {linked }}$ obtaining once more a binarized image $B I_{\overline{C e l l}}$. In this final binarized image of the cell we determined the mean distance between the center of the cell area and the cell contour and choose this parameter to be $R$ (figure IV.12).

\section{IV.8 Processing of Images from Stained Platelets}

As mentioned before (section I.3 and chapter III) force measurements on platelets were complemented by separate staining experiments of platelet activation both on glass and soft PAA substrates. Images taken on glass were spectrally unmixed using a plugin in the freeware ImageJ by Joachim Walter (Bio-Imaging Center, LudwigMaximillian University München). For staining on PAA, in turn, spectral unmixing was performed with the software xcellence rt (Olympus, Tokio, Japan). While images on glass were taken with a high resolution confocal setup, images on PAA were acquired by conventional epifluorescence microscopy (section III.6). Hence, images on PAA were a little blurred in appearance. To reduce blurring, we therefore applied a no neighbor filter with a haze removal factor of $50 \%$ (xcellence rt software) to each image on PAA.

In the final images we then went on to identify representative cells with typical staining patterns for the different stages of platelet activation both on glass and PAA. For the particular case of PAA, we compared the morphologies of stained platelets with those seen during platelet force measurements, to assist us in our cell selection (figure IV.13). By matching morphologies, we were thus able to relate platelet force development on PAA to both the reorganization of platelet cytoskeletal components as well as the final distribution of focal adhesions. More details on our results will be given in the following chapter $\mathrm{V}$.

\footnotetext{
${ }^{8}$ This corresponds to the time point in the steady state of force generation mentioned in section V.1.1
} 


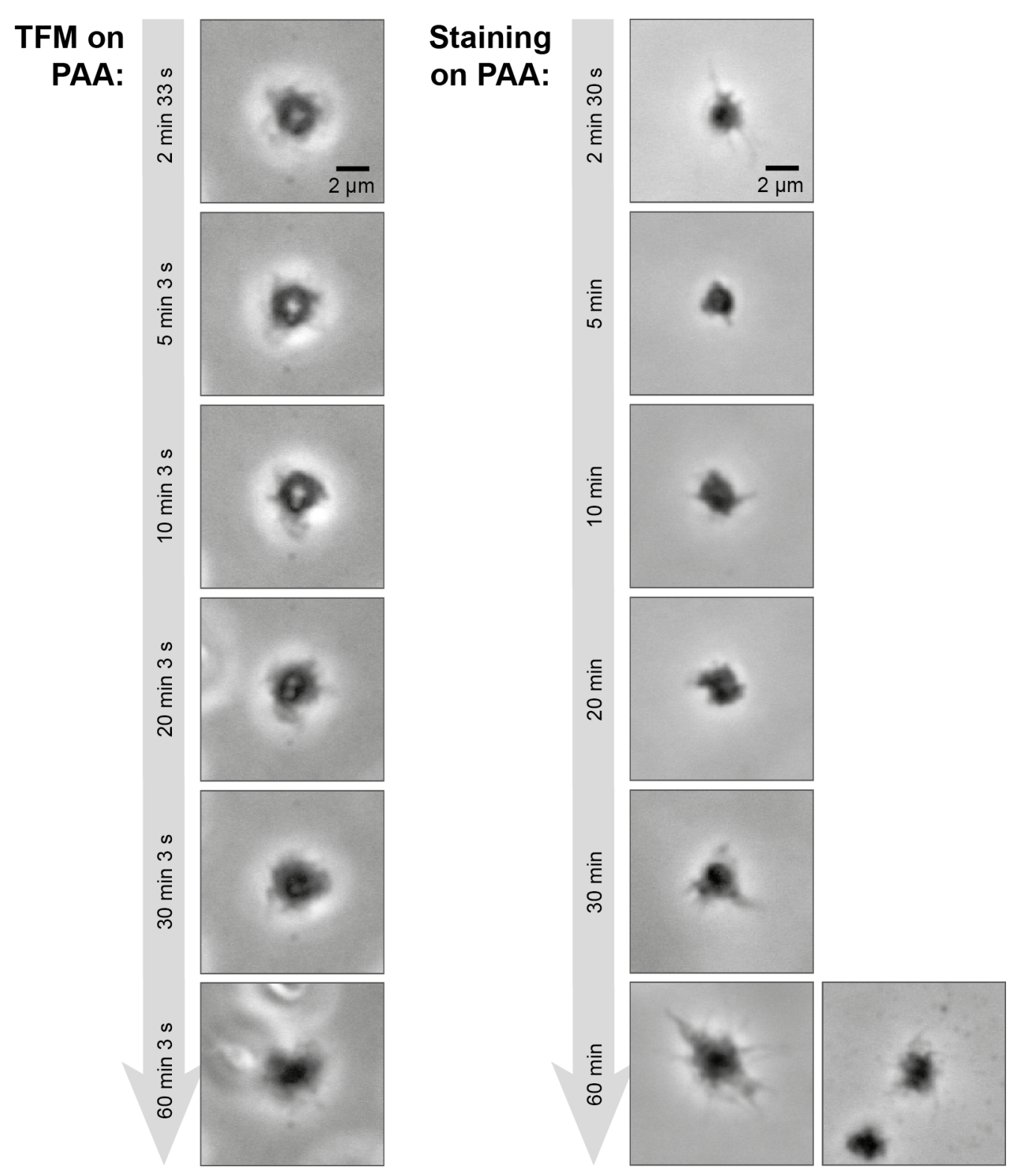

Figure IV.13: By comparing platelet morphologies, we were able to relate staining experiments to TFM force measurements, which were, though separately, both performed on platelets that were activated on PAA substrates: Representative staining patterns for platelet activation on PAA (see sections V.1.2 and V.2.2) were selected based on a comparison of observed platelet morphologies (images on the right) with typical morphologies seen during traction force experiments (example shown on the left). 


\section{Results}

In section I.4 we have introduced our experimental technique, traction force microscopy (TFM), for measuring platelet forces. Following each TFM recording, we obtain a movie of traction force fields for single platelets (chapter IV]), showing how forces gradually develop during platelet contraction on soft polyacrylamide (PAA) substrates. An example of snapshots taken from one of these movies is given in figure V.1. A qualitative
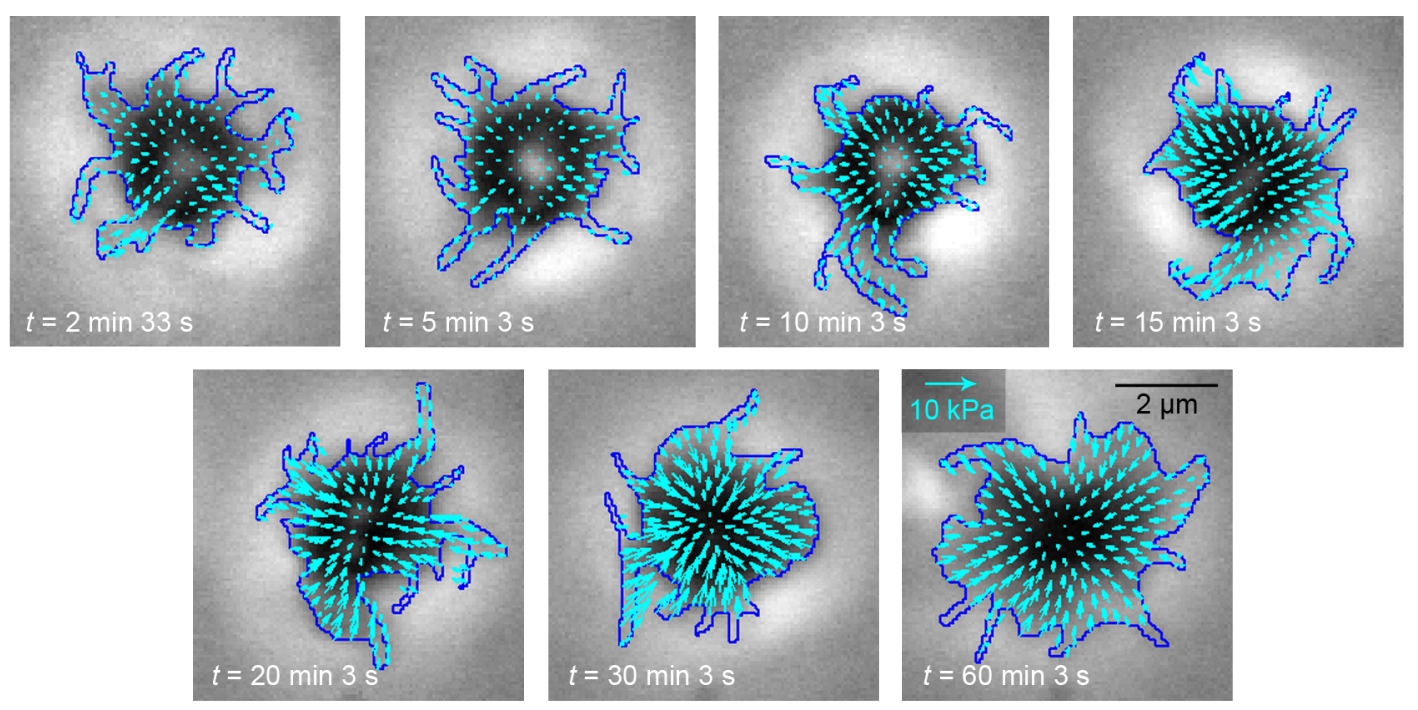

Figure V.1: Snapshots taken from a typical movie showing traction force field evolution in a single, contracting platelet: Phase contrast images of a single platelet during contraction on a soft PAA substrate were overlaid with the traction forces that the platelet generated. Traction forces were calculated based on a TFM recording of the platelet.

inspection of the snapshots already gives a first impression of the characteristics of platelet contraction. Thus, we see that after a certain time a stable traction force field emerges. It is also noticeable that traction forces are largest at the periphery pointing seemingly isotropically toward the cell center. All these characteristics can be categorized as aspects describing the temporal evolution or the spatial distribution of traction forces. In the subsequent section we address these two categories separately and proceed with a quantitative analysis of platelet contraction. The figures presented in this context are mostly taken from one of our manuscript:[1], which has recently been published [53. We have complemented these figures on platelet forces with fluorescence snapshots of platelets that were fixed and stained at different stages during

\footnotetext{
1 These include figures V.2, $\mathrm{V} .3, \mathrm{~V}, \mathrm{~V}, \mathrm{~V} .7$. The figures have only been slightly changed by introducing explanatory sketches and additional data insets. The snapshots in figure V.1 are taken from a movie shown in the online supplement of our paper.
} 
contraction on PAA (section III.5). By comparing the figures and snapshots, we are able to relate TFM observations to cytoskeletal reorganization and pattern formation. To describe our results we use the notation $\bar{X}$ to indicate a temporal average of any variable $X$ and $\langle X\rangle_{N}$ to describe the corresponding ensemble average.

\section{V.1 Temporal Evolution of Platelet Contraction}

\section{V.1.1 Traction Force Evolution}

Our qualitative inspection of TFM data has already indicated that activated platelets generate a stable traction force field after a certain time. Our first approach to quantitatively describing the evolution of platelet contraction is therefore to assess how long it typically takes for platelets to establish this force field. To that end, we extract time tracks of the total forces $F_{t o t}(t)$ generated by single platelets from each TFM recording. The total force $F_{t o t}(t)$ for a given time frame is related to the traction force field $\vec{T}(\vec{x}, t)$ in the TFM data set by an integration over the cell area: $F_{\text {tot }}(t)=\int_{A}|\vec{T}(\vec{x}, t)| \mathrm{d} A^{[2]}$. To mark the beginning of the time track, we set $t=0 \mathrm{~s}$ to the time point of cell adhesion to the substrate (see section IV.5). A typical time track of $F_{t o t}(t)$ for a single platelet that we thus obtain is shown in figure V.2 a). We find that the forces increase linearly at the beginning of platelet activation $(t<1798 \mathrm{~s})$, before the cell reaches a steady state at $F_{\text {tot }}^{S t} \sim 40 \mathrm{nN}$, in which cellular contractile forces are balanced against the elastic forces of the substrate. The turning point $t_{t p}$ at $1798 \mathrm{~s}$ between force increase region and steady state region is also clearly visible in the example shown in figure $\mathrm{V} .2 \mathrm{a}$ ).

To compare typical values for $F_{t o t}^{S t}$ and $t_{t p}$ for a statistical ensemble, we fit the following composite of linear functions with OriginPro 8.5G (user defined fit function; OriginLab Corporation, Northampton (MA), USA) to both force generation regions in the time tracks of $F_{t o t}(t)$ :

$$
f(t)= \begin{cases}A \cdot t+y_{0}, & t<t_{t p} \\ A \cdot t_{t p}+y_{0}, & t \geq t_{t p}\end{cases}
$$

where $A, t_{t p}$ and $y_{0}$ are set as the fit parameters. For each platelet we extract a value for $t_{t p}$ and $F_{t o t}^{S t}=A \cdot t_{t p}+y_{0}$ from the linear fits, and plot their relative abundance for an ensemble of 14 cells from 4 different cell preparations (figure V.2). However, the fit according to equation V.1 has proved impossible for one of the TFM data sets. Instead a constant to the constant region in $F_{\text {tot }}(t)$ has been fitted so that this cell appears in the histogram for $F_{t o t}^{S t}$, but is absent in the one for $t_{t p}$. We find that on average platelets display a steady state total force of $\left\langle F_{t o t}^{S t}\right\rangle_{N} \sim 34 \mathrm{nN}$ a time $\left\langle t_{t p}\right\rangle_{N}=25 \mathrm{~min}(1500 \mathrm{~s})$ after cell adhesion to the substrate. Also, we see that the distribution for $F_{t o t}^{S t}$ is asymmetric, extending a tail toward larger values. We assume the asymmetry to be related to the different sizes of the analyzed platelets. To verify this hypothesis we plot $F_{t o t}^{S t}$ against the time-averaged steady state cell area $\bar{A}$. This average cell area $\bar{A}$, in turn, we

\footnotetext{
${ }^{2}$ Traction forces $\vec{T}(\vec{x})$ are after all local forces $\vec{F}(\vec{x})$ per unit area $\mathrm{d} A: \vec{T}(\vec{x})=\vec{F}(\vec{x}) / \mathrm{d} A$.
} 
a)

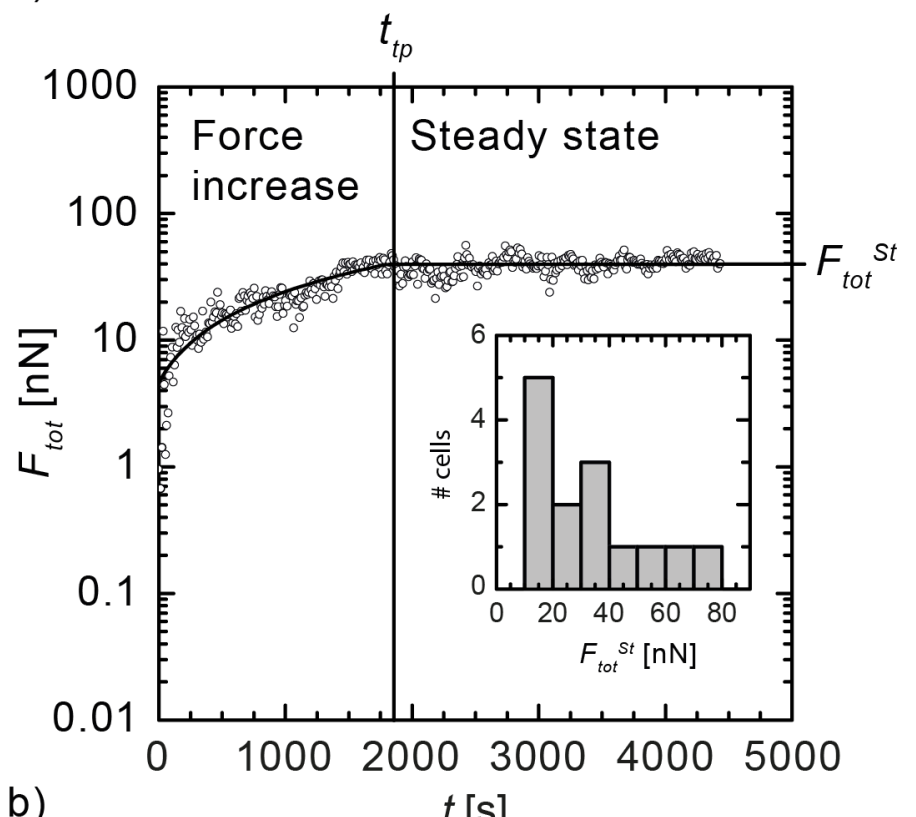

b)

$t[\mathrm{~s}]$

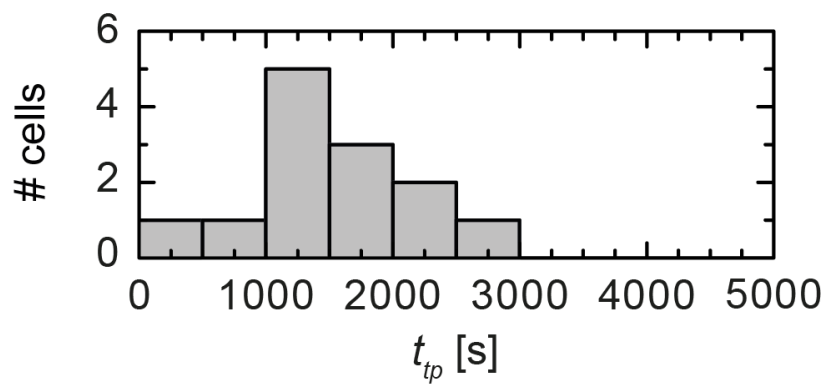

Figure V.2: Platelets reach a steady state after $\sim 25 \mathrm{~min}$ and display large contractile forces: a) Time course of the total force $F_{\text {tot }}$ generated by a single blood platelet after cell adhesion to a polyacrylamide (PAA) substrate plotted on a logarithmic scale for a typical traction force microscopy (TFM) data set. Linear fits to both force increase region $(<1798 \mathrm{~s})$ and steady state region $(\gtrsim 1798 \mathrm{~s})$ are displayed as solid black lines. From the linear fits the turning point $t_{t p}$ between the two regions of force generation and the total steady state force $F_{t o t}^{S t}$ are obtained. The inset and b) show the statistical distribution of values for $F_{t o t}^{S t}$ and $t_{t p}(N=14$ and $N=13$ cells, respectively). 


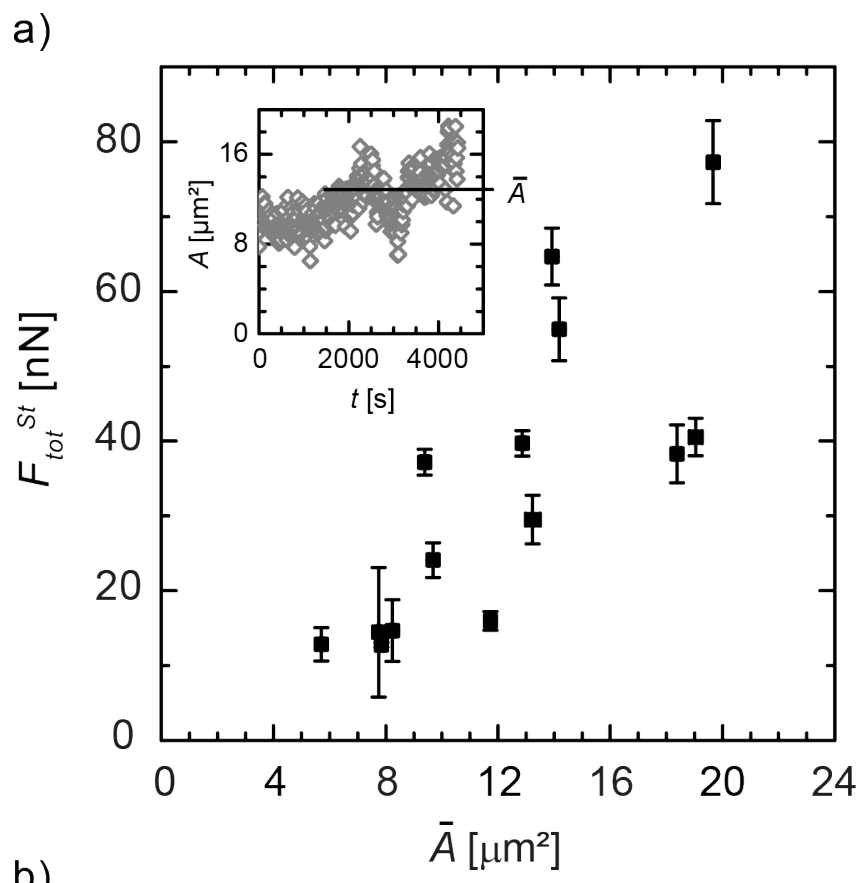

b)

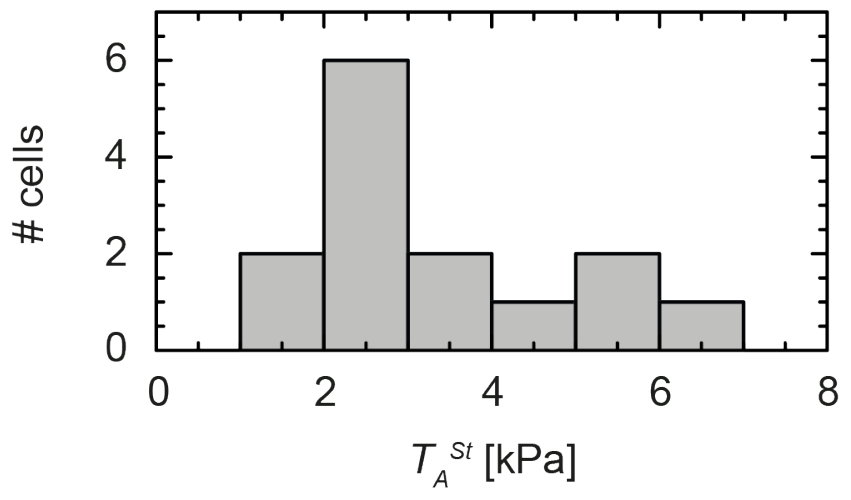

Figure V.3: Total contractile force depends on platelet size: a) Steady state total force $F_{\text {tot }}^{S t}$ generated by individual blood platelets (error bars show the fit error) plotted against the time-averaged cell area $\bar{A}$ (standard error of mean is smaller than the symbols). $\bar{A}$, in turn, is extracted for each cell from time tracks of the cell area such as the example shown in the inset. b) Distribution of traction forces $T_{A}^{S t}$ averaged over the cell area $A$ at the steady state $(N=14$ cells $)$.

obtain by averaging for each $t \geq t_{t p}$ the time tracks of the cell area $A$, which we obtain from the cell contours in the TFM movies (inset in figure V.3 a)). As figure V.3 a) shows, the resulting graph confirms our initial assumption, and the extending tail in the distribution for $F_{t o t}^{S t}$ originates indeed from a few larger platelets that generate larger traction forces. To reduce the influence of platelet size on our results, we also look at the traction force averaged over the cell area $\langle|\vec{T}(\overrightarrow{x, t})|\rangle_{A}=1 / A \int_{A}|\vec{T}(\vec{x}, t)| \mathrm{d} A$ and extract for each platelet the corresponding steady-state value $T_{A}^{S t}$. A histogram summarizing the distribution of $T_{A}^{S t}$ for our cell ensemble is shown in figure $\mathrm{V} .3 \mathrm{~b}$ ). Here, the ensemble-average is $\left\langle T_{A}^{S t}\right\rangle_{N}=3.3 \mathrm{kPa}$. 


\section{V.1.2 Cytoskeletal Reorganization}

After having established that it takes platelets about 25 min to generate maximum contractile forces on PAA substrates, we sought next to understand what underlying mechanisms determine the 25 min time span. To achieve this goal, we fixed platelets in a separate experiment at certain time points during activation on PAA and stained filamentous actin (F-actin) ${ }^{[3]}$ For control purposes, we repeated the experiment using glass cover slips as substrates. Here, we added green fluorescent beads $(0.2 \mu \mathrm{m}$ in diameter) to also visualize platelet internal contraction, that is, to see the centralization of platelet granules during activation $[4]$.

A summary showing typical actin structures for both experiments is displayed in figure V.4. One of the first features that is noticeable in figure V.4 is that initially F-actin fluorescence is homogeneously distributed across the platelets for both PAA and glass substrates. Following this state, actin reorganization seems to occur predominantly at the cell periphery. Thus, for both PAA and glass substrates we see an elevated concentration of F-actin at the edge of the cell body about 5 min after platelet activation was triggered. While for PAA elevated F-actin concentrations are found exclusively at the edge of the cell body, on glass surfaces we see additional F-actin streaks radiating into the cell body from a particular spot at the cell periphery. These F-actin streaks gradually develop into stress fibers that form distinct patterns on glass. Similarly, we also observe pattern formation on PAA, although here the stress fibers are less distinctive and there appears to be no preferred angle of orientation. A rigorous characterization of the actin structures on PAA, however, turns out to be difficult, because images look a little blurred specially in a comparison with the images taken on glass. The main reason for the difference in image quality lies in the fact that snapshots on PAA were taken by conventional epifluorescence microscopy, while image acquisition on glass was performed by high-resolution confocal microscopy (section III.6). Nevertheless, both on PAA and glass we see that a remarkable actin network develops in the first half hour of activation. During this time or perhaps even within the first 15 min of activation, internal contraction is completed, as indicated by the centralized bead clusters in platelets on glass (discussed in more detail in section VII). Interestingly, the 30 min time span that it takes platelets to develop the actin network seen in figure V.4 correlates with the force increase region during TFM experiments.

\footnotetext{
${ }^{3}$ As mentioned in section I.3. we choose this indirect approach of imaging cytoskeletal reorganization, because platelets cannot be transfected by standard techniques to fluoresce while living.

${ }^{4}$ As indicated by reference 32 platelets take up latex beads and store them in their granules. We added the beads here as a test to see whether bead uptake relates to cytoskeletal rearrangement and might be used in future experiments to observe the cytoskeletal dynamics in living cells (see section VII).
} 

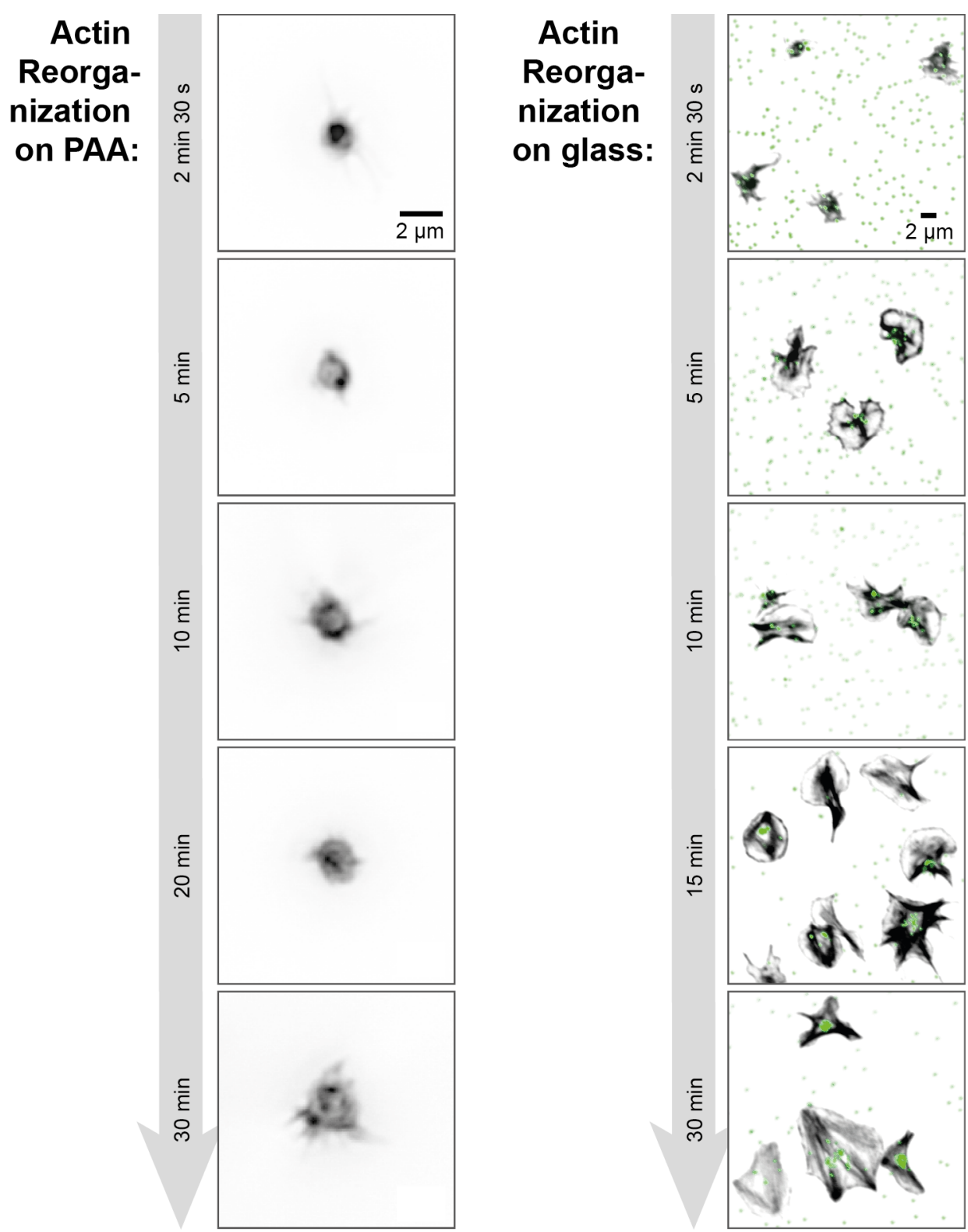

Figure V.4: Stress fiber formation and stress fiber patterning is more distinctive during platelet activation on glass as opposed to activation on PAA: Platelets were fixed and stained for filamentous actin at different stages during thrombin-triggered platelet activation on fibrinogen-coated $4 \mathrm{kPa}$ PAA substrates (left-hand side) or on equally coated glass cover slips (right-hand side; see also section III.5). For platelets on PAA, cells were permeabilized prior to staining to maximize staining efficiency, and samples were mounted on microscope slides to improve imaging. For platelets on glass, in turn, a solution of fluorescence beads $(0.2 \mu \mathrm{m}$ in diameter, shown in green) was added before triggering activation in order to visualize platelet internal contraction (section I.1.2, p. 10). Images were acquired at an epifluorescence (PAA) and a confocal setup (glass). Typical staining patterns, shown in inverted grayscale values, depict actin reorganization into a network during platelet activation on both substrates. 


\section{V.2 Spatial Organization during Platelet Contraction}

\section{V.2.1 Distribution of Traction Forces}

As mentioned before (section I.3 and I.4), one of the great advantages of TFM is that it enables us to resolve platelet forces spatially as well as temporally $\left[{ }^{[5]}\right.$. As far as we know, the recording of 2-D traction force maps, in particular, is a novelty for platelet contraction. To fully take advantage of TFM's potential, we therefore characterize the spatial distribution of platelet traction forces by determining the focus point of the traction force field $\vec{T}(\vec{x})$ and by quantifying the degree of isotropy of the fields. The first point we address by calculating the absolute divergence minimum $\min (\vec{\nabla} \cdot \vec{T}(\vec{x}, t))$ for each time frame $t$ in a TFM recording (figure V.5 a) and b)). To reduce the influence of noise on $\min (\vec{\nabla} \cdot \vec{T}(\vec{x}, t))$, we smooth $\vec{T}(\vec{x}, t)$ before calculating the divergence of the traction force field (section IV.7.1). For each time frame $t$ that the platelet is in the steady state of force generation, we then plot the position of $\min (\vec{\nabla} \cdot \vec{T}(\vec{x}, t))$ relative to the center of the cell area. This involves adjusting the coordinate system for each $t$ so that the center of the cell area always coincides with the origin of the coordinate system $\left(x_{1}=0, x_{2}=0\right)$. The graphs that we thus obtain for each cell, however, differ visibly (see figure V.5 c) and d)). For some cells, the plot has a cloud-like shape. Here, the position of $\min (\vec{\nabla} \cdot \vec{T}(\vec{x}, t))$ scatters little over time around its average position. For other cells, by contrast, the point $\min (\vec{\nabla} \cdot \vec{T}(\vec{x}, t))$ seems to move on a circle or semicircle around the center of the cell area. To compare platelets nonetheless, we consider the distance $d$ between the position of $\min (\vec{\nabla} \cdot \vec{T}(\vec{x}, t))$ and the center of the cell area in the steady state of platelet contraction (see figure V.5 b). For each individual platelet we calculate the time average of $d(\bar{d})$ and normalize it with the typical cell radius $R$ (see also section IV.7.2). We thus obtain a distribution of $\bar{d} / R$ for a cell ensemble of $N=14$ cells (figure V.5 e)). In this distribution a peak is discernible below 0.5, confirming our first impression that on average contractile forces in platelets are directed toward a point near the center of the cell area.

Our first impression from the inspection of the movies of traction forces $\vec{T}(\vec{x}, t)$ has further indicated that steady state platelet contraction is isotropic. To quantitatively assess the degree of isotropy, we approximate the field at each time point $t$ for a single cell with an overlay of two force dipoles and look at the force dipole moments $D_{ \pm}(t)$ corresponding to the major and minor axis of contraction (figure V.6 a), see also section IV.4). As before, we fit a combination of linear functions to $D_{ \pm}(t)$ (see equation V.1) and extract the constant $D_{ \pm}^{S t}$ for the steady state regime (figure V.6 b)). As an example, figure V.7 a) shows the distribution of $D_{-}^{S t}$ for the minor axis of contraction in our ensemble of 14 cells. We see again an asymmetric distribution, with an average of $\left\langle D_{-}^{S t}\right\rangle_{N} \sim-20 \mathrm{nN} \mu \mathrm{m}$. Here, the negative sign of the dipole moment demonstrates that platelets generate contractile rather than pushing forces. As before, we find that the asymmetry in the distribution of $D_{-}^{S t}$ is related to platelet size (inset in figure V.7 a), see also section V.1.1). Interestingly, when plotting a histogram of the ratio between $D_{+}^{S t}$ and $D_{-}^{S t}$, we perceive a peak around 1.25 (figure $\mathrm{V} .7 \mathrm{~b}$ )).

\footnotetext{
5 To be able to evaluate long temporal records of platelet contraction, we have had to make a few adjustments to the TFM technique (see sections I.4 and IV.3.
} 
a)

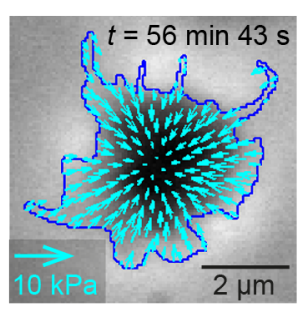

c)

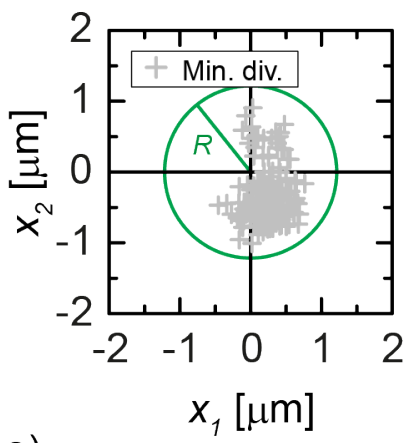

b)

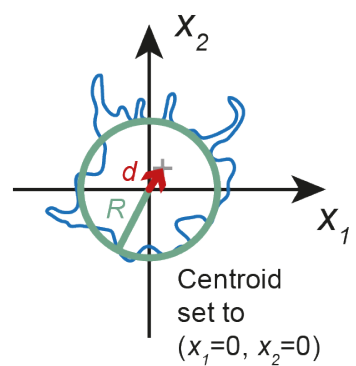

d)

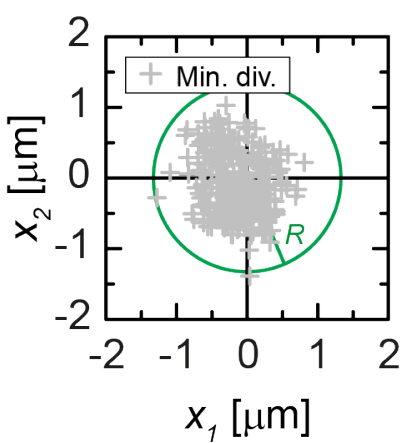

e)

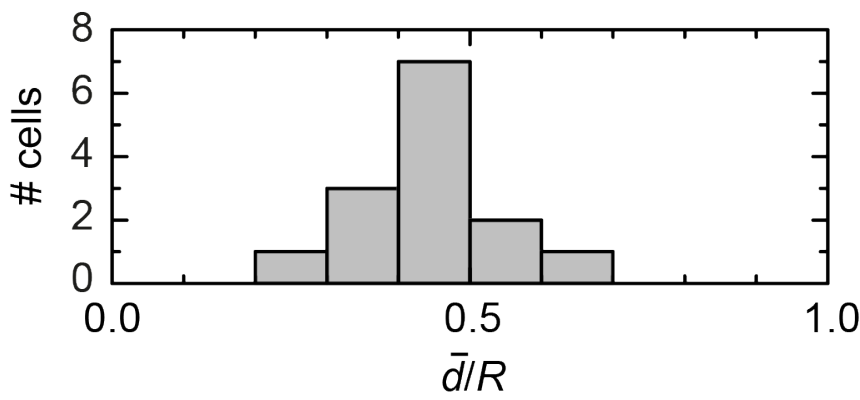

Figure V.5: Platelets pull toward a point near the center of the cell area: a) Typical image of the traction force field $\vec{T}(\vec{x}, t)$ at some time point $t$ in the steady state of platelet contraction. b) For each $t$ in the steady state, the position of the divergence minimum $\min (\vec{\nabla} \cdot \vec{T}(\vec{x}, t))$ is extracted relative to the center of the cell area $\left(x_{1}=0, x_{2}=0\right)$ after reducing noise in $\vec{T}(\vec{x}, t)$. In addition, the distance $d$ of $\min (\vec{\nabla} \cdot \vec{T}(\vec{x}, t))$ to $\left(x_{1}=0, x_{2}=0\right)$ is inferred. The cell images in the TFM movies are furthermore used to estimate a typical cell radius $R$ for each individual cell. c) and d) Example plots of two different platelets, in which all positions of $\min (\vec{\nabla} \cdot \vec{T}(\vec{x}, t))$ were collected over time. e) Histogram showing the relative abundance of $\bar{d} / R$ for an ensemble of $N=14$ cells, where $\bar{d}$ represents the time average of the distance $d$. 
a)

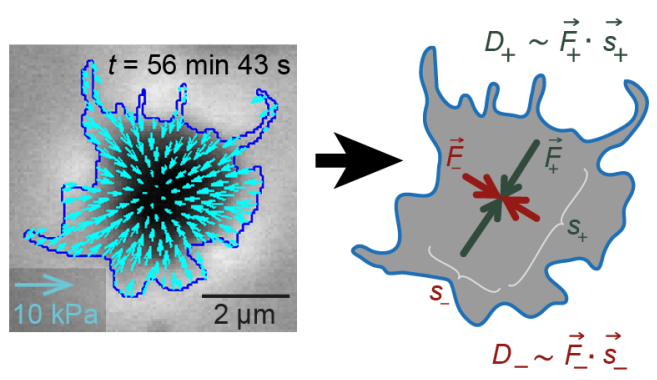

b)

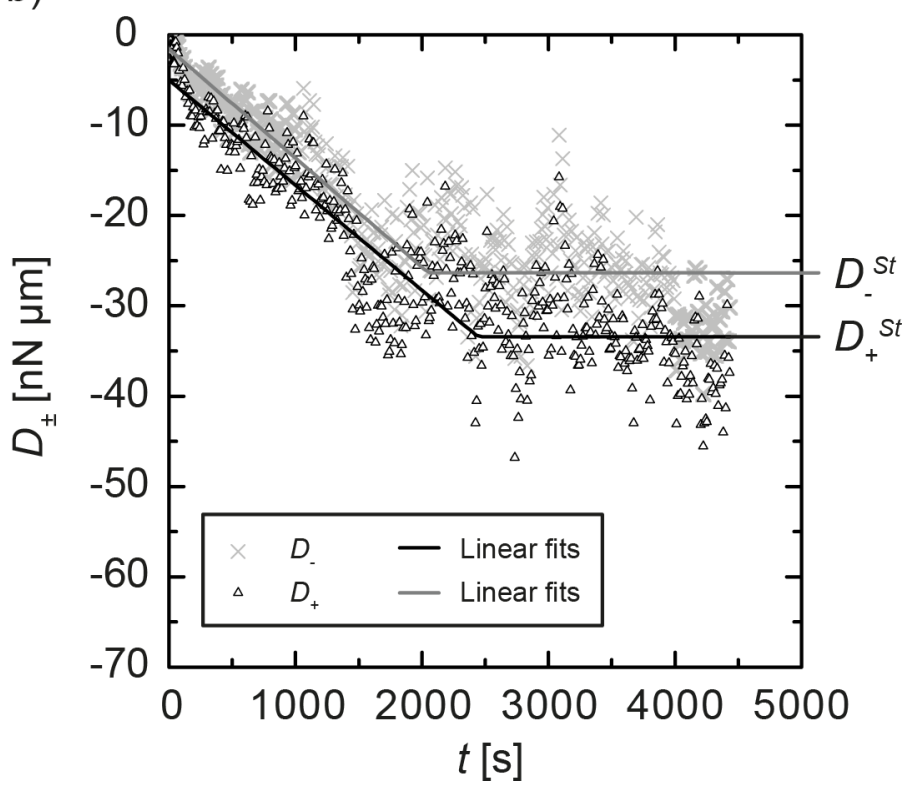

Figure V.6: A force dipole approximation is used to determine the degree of isotropy of platelet contraction: a) Traction force fields at each time $t$ are approximated by two force dipoles that align with the major and minor contractile axes. For both dipoles, the force dipole moment $\left(D_{+}\right.$and $D_{-}$, respectively) is calculated. b) Typical time track of $D_{+}$and $D_{-}$for one platelet. A combination of linear functions is fitted to each time track yielding the steady state dipole moments $D_{+}^{S t}$ and $D_{-}^{S t}$.

This indicates that the difference between major and minor axis of contraction is small, and we conclude that steady state platelet contraction is indeed nearly isotropic.

\section{V.2.2 Distribution of Myosin Motors and Focal Adhesions}

In the previous section we have demonstrated with our TFM data that platelets pull nearly isotropically toward a point close to the cell center. Following such an observation, the question naturally arises how platelets are able to perform isotropic contraction on PAA substrates. To address this point, we carried out two additional experiments with fixed and fully activated platelets on PAA substrates, where we stained F-actin as well as focal adhesion vinculin and non-muscle myosin IIA motors. In the first experiment, we simultaneously stained only the first two components. The PAA substrates for this experiment were prepared without the fluorescent beads that are used for TFM to avoid interfering fluorescence signals. The second experiment 
a)

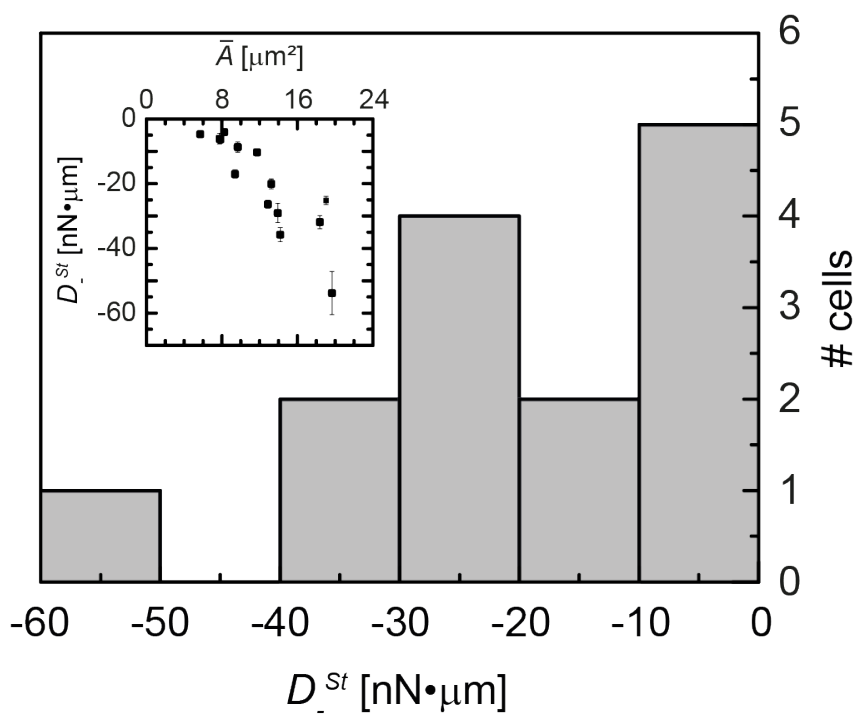

b)

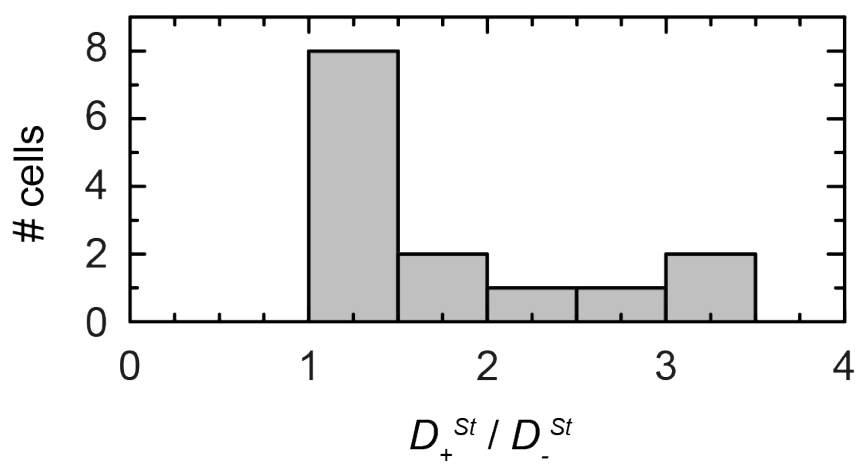

Figure V.7: Platelets pull nearly isotropically, the difference between major and minor axis of contraction is small: a) Distribution of the steady state force dipole moment $D_{-}^{S t}$ for the minor axis of contraction according to fits such as the one depicted in figure V.6 b). The inset shows the dependence of $D_{-}^{S t}$ (error bars represent the fit error) on the time averaged cell area $\bar{A}$ (standard error of mean is smaller than the symbols). b) Histogram for the ratio of the steady state force dipole moment of the major $\left(D_{+}^{S t}\right)$ and minor $\left(D_{-}^{S t}\right)$ contractile axis for the individual cells $(N=14)$. 


\section{Phase contrast image}

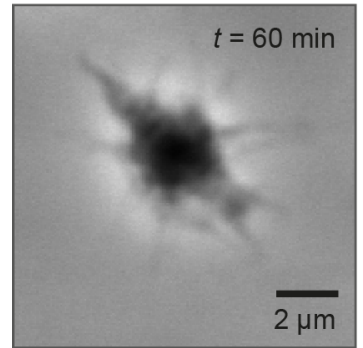

Vinculin

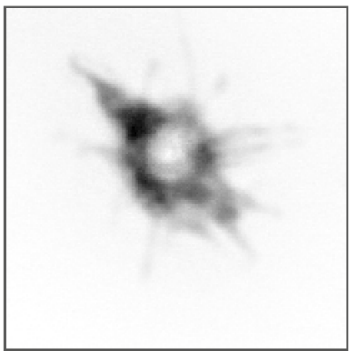

F-actin

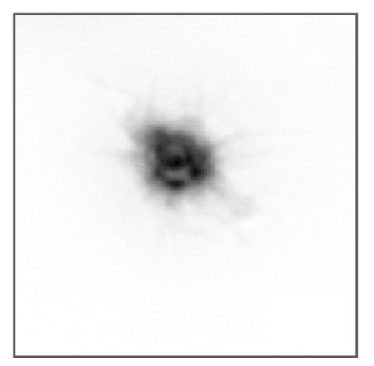

Vinculin + Actin

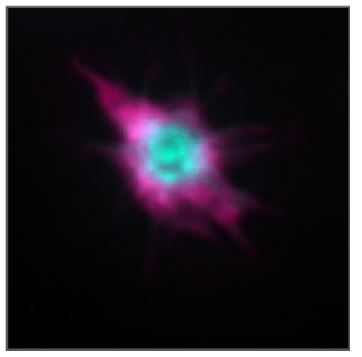

Figure V.8: During platelet activation on PAA, F-actin concentrates mostly at the cell center, while focal adhesions form primarily at the periphery: Platelets were fixed and stained for filamentous actin after $60 \mathrm{~min}$ thrombin-triggered activation on fibrinogen-coated $4 \mathrm{kPa}$ PAA substrates (see also section III.5). Additionally, the focal adhesion protein vinculin was stained. Platelets were permeabilized prior to staining to maximize staining efficiency and stained samples were mounted on microscope slides to improve imaging. Staining patterns for each actin and vinculin were recorded using epifluorescence microscopy, typical images of which are shown in inverted grayscale values. Furthermore, a colored overlay of the single fluorescence images of both actin and vinculin is depicted on the right-hand side, setting the spatial distribution of the two proteins into relation.

involved a triple staining of all three components. Here, the PAA was accidentally prepared with fluorescent beads that were, by happenstance, of the same color as the vinculin staining. Nevertheless, the presence of the beads turned out to be advantageous, as focusing on the PAA surface was facilitated by their presence. Typical staining patterns for the two experiments are shown in figures V.8 and V.9.

Both experiments show the same qualitative distribution for vinculin and actin. That is, we find that actin concentrates in the center of the cell area, whereas vinculin is found primarily at the periphery of the cell. It should be noted, however, that weak actin fluorescence is also seen in filopodia and at the edge of the cell (figure V.8, third image from the left). Therefore, actin filaments or thinner actin bundles are still present in these regions, although they are barely visible in the fluorescence images due to their small thickness. Interestingly, we find that actin patterns are not perfectly matched by myosin fluorescence signals. Instead we see in our second staining experiment that myosin forms a ring-like structure in between the bulk of the actin in the cell center and the vinculin lining the edge of the cell (figure V.9). Within this ring-like structure, we observe elevated myosin concentrations at the root of filopodia. By contrast, myosin fluorescence is absent along the remaining length of the filopodia. The significance of these findings and possible implications for force generation in activated platelets on PAA are discussed in the following chapter (section VI.4). 


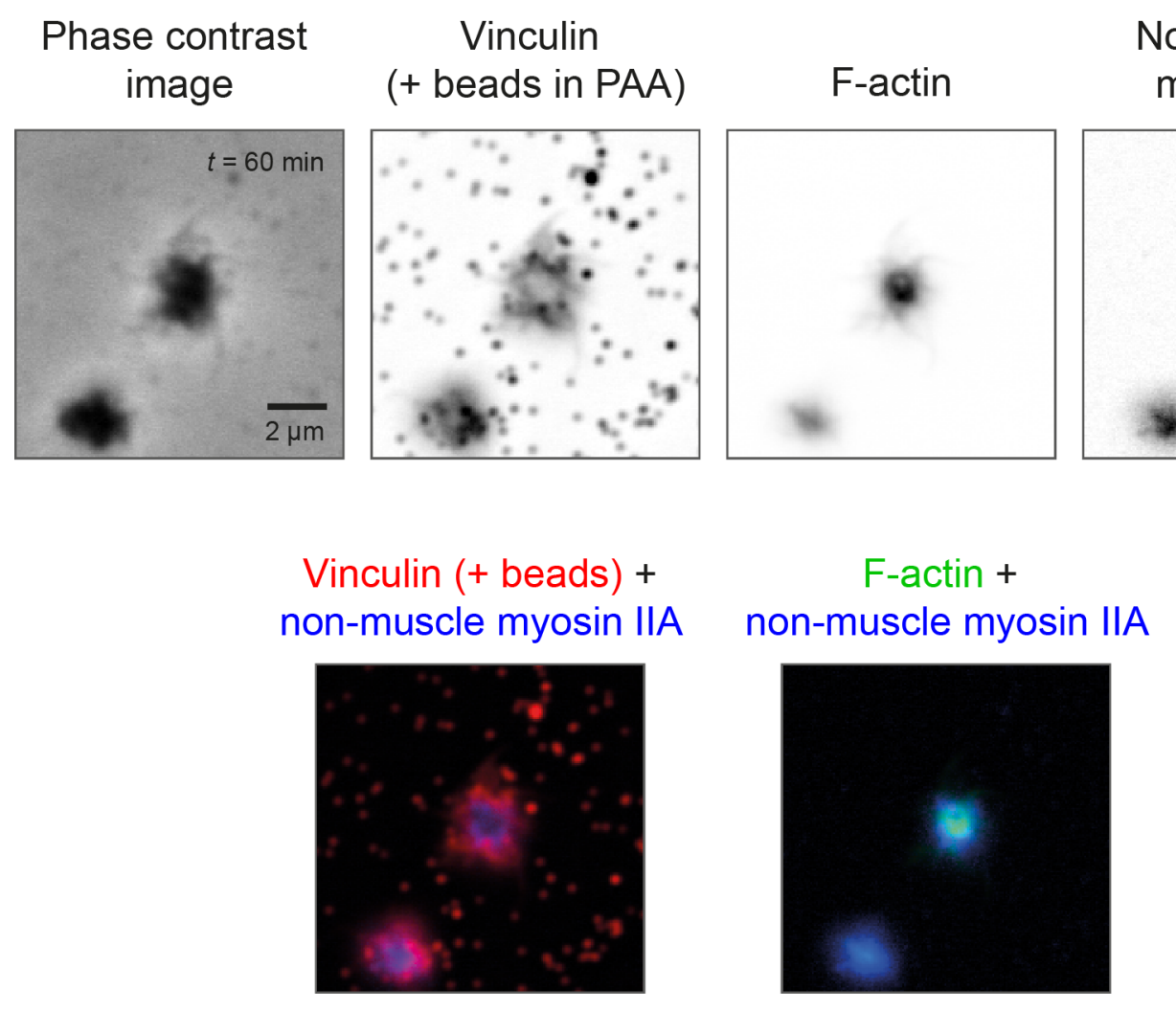

Figure V.9: Myosin IIA motors form a ring-like structure around the cell center and also localize to the root of filopodia during platelet activation on PAA: Platelets were activated on PAA, fixed, permeabilized, stained for actin and vinculin and mounted as described in figure caption V.8. Here, however, red fluorescent beads $(0.1 \mu \mathrm{m}$ in diameter) were incorporated into the PAA substrate at the top surface, which facilitated focusing on the cell adhesion plane. Also, non-muscle myosin IIA motor proteins were stained in addition to actin and vinculin (section III.5). As before, images were taken with an inverted epifluorescence setup, typical patterns for the different stainings are shown as inverted grayscale images. Additionally to the single fluorescence snapshots, the figure displays colored overlays of myosin staining in combination with either actin or vinculin to show their relative spatial organization. 


\section{Discussion and Conclusions}

In the previous chapters we have presented our study of cellular contraction using the example of human blood platelets and explained our two experimental approaches. One consists of force measurements on single platelets using traction force microscopy (TFM). For the other one, we fixed and stained platelets during different stages of activation on soft PAA substrates to visualize cytoskeletal reorganization and the distribution of focal adhesion sites. In combining these two approaches, we aim to obtain more detailed information about mechanical principles of force generation and transduction in platelets. Our further objective is to identify features that platelets share with other cells generating contractile forces. To this end, we will first compare our measurements to previous studies on platelet contraction (section VI.2). Then, we will estimate to which degree different mechanisms of force generation contribute to our measured platelet forces (section VI.3). Following this, we will discuss different theoretical, mechanical models for adherent cells and evaluate their applicability to the special case of platelets (section VI.4). Finally, we will compare the contraction of platelets with other cellular contractile processes (section VI.5). Before we interpret our findings, however, we will consider experimental errors in our measurements (section VI.1).

\section{VI.1 Limitations of TFM}

To discuss experimental errors in TFM recordings, we need to return to particulars of the TFM technique. As described in sections I.4 and III.4, during our TFM experiments platelets are seeded on elastic PAA substrates, which they deform as soon as they start generating contractile forces. These substrate deformations are visualized with the help of fluorescent marker beads within the PAA, which are tracked over time by fluorescence microscopy. Simultaneously, platelets are imaged by phase contrast microscopy to obtain the cell-substrate contact area $A$, over which force transduction can occur. From the bead displacements $d_{j}\left(\vec{x}^{\prime}\right)$, traction forces are calculated for the area $A$ by solving the following equation:

$$
d_{j}\left(\vec{x}^{\prime}\right)=\int_{A} G_{i j}\left(\vec{x}^{\prime}-\vec{x}\right) T_{i}(\vec{x}) d x_{1} d x_{2}
$$

where $G_{i j}$ is Boussinesq's approximation describing the elastic response of the substrate (section II.1), and $T_{i}(\vec{x})$ are the traction forces exerted by the cells. From the above equation it is already possible to deduce potential sources of errors. Thus, experimental errors are either introduced by the estimation of the cell area $A$ or by the detection of bead displacements $d_{j}\left(\vec{x}^{\prime}\right)$. Errors of computation that result from inverting the integral in the above equation are added to these experimental errors. Finally, some degree of 
inaccuracy may also be introduced by using Boussinesq's approximation. Fact is that the Boussinesq solution is only truly valid for substrates that are infinitely thick.

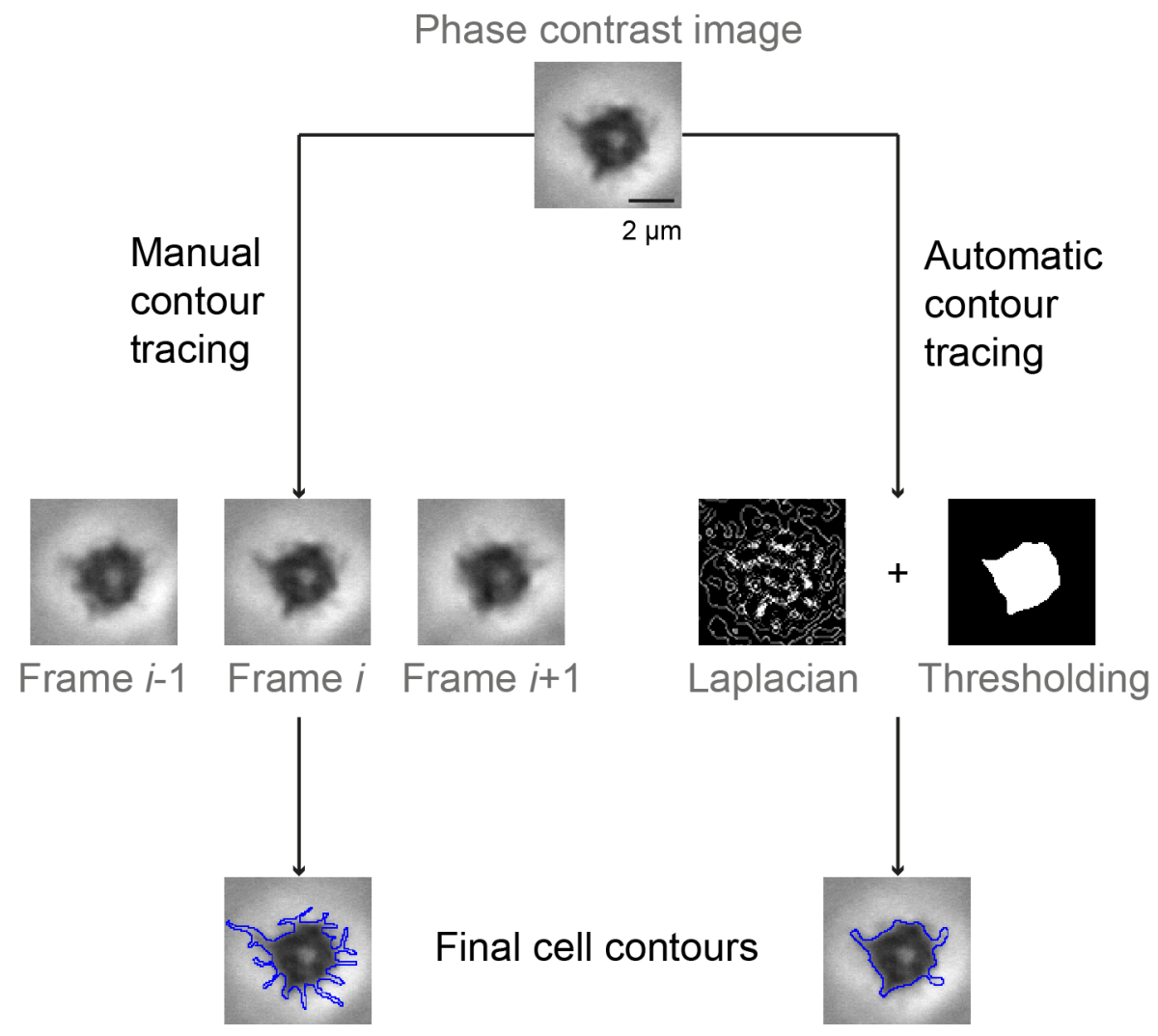

Figure VI.1: Comparison of automatic cell contour tracing with manual contour tracing: In one example of a TFM data set, cellular phase contrast images were traced both manually and fully automatically (estimated contours are shown as blue lines). Automatic tracing involved using a Laplacian filter on the original image. The result was then combined with a binarized form of the original image obtained by thresholding (see section IV.3). During manual tracing, each image at a time $t$ was compared with the previous and the following time frame in order better to estimate the position of filopodia and lamellipodia. [Figure from our publication [53]]

We begin by considering the experimental error introduced by the estimation of $A$. As described in section IV.3 we developed a program to extract $A$ from the large amount of recorded data. The program encountered difficulties in tracing platelet contours when the contrast in the image became too poor on account of parts of the platelets becoming too thin. That is, the program had difficulties in tracing filopodia and lamellipodia. To estimate the error introduced by insufficient contour detection, we picked a data set for which we redrew contours manually for each time frame $t$ in the TFM recording (total of 471 frames). In this process, we compared each time frame $t$ with the previous time frame $t-1$ and the following time frame $t+1$ in order better to estimate the position of filopodia and lamellipodia and further to reduce the influence of noise (figureVI.1). With these manual corrections, we recalculated traction forces for the data set and compared the new results with the previous ones. The results with automatically traced contours show a deviation relative to the ones with manually 
traced contours of: $\Delta F_{\text {tot }}^{S t} / F_{\text {tot }}^{S t}=-12.8 \%, \Delta T_{A}^{S t} / T_{A}^{S t}=+6.4 \%, \Delta D_{-}^{S t} / D_{-}^{S t}=+21.2 \%$ and $\Delta\left(D_{+}^{S t} / D_{-}^{S t}\right) /\left(D_{+}^{S t} / D_{-}^{S t}\right)=-1.4 \%$. Here, $F_{t o t}^{S t}$ is the total force, $T_{A}^{S t}$ is the average traction force, $D_{+}^{S t}$ is the force dipole moment of the major axis of contraction and $D_{-}^{S t}$ is the corresponding dipole moment of the minor axis of contraction (sections V.1.1 and V.2.1). All four parameters relate to the steady state of platelet contraction. This error estimate demonstrates that our total forces $F_{\text {tot }}$ are generally underestimates, whereas our average traction forces $T_{A}^{S t}$ and our force dipole moments $D_{ \pm}$are overestimates. The effect on $T_{A}^{S t}$ is only minor and the effect on $D_{ \pm}$cancels out in the ratio $D_{+}^{S t} / D_{-}^{S t}$.

We next turn to the discussion of errors introduced by the detection of bead displacements. In our approach, bead displacements were calculated from the fluorescence images of the beads by using a particle tracking approach (see section IV.2). Particle tracking is, however, known to have its disadvantages [7]. For example, bead identification becomes difficult in clusters, bead matching is challenging at high bead densities, and in areas of low bead density the spatial resolution is reduced. Nevertheless, we chose this approach over the correlation technique more commonly used in the community (section I.4), as bead patterns change too rapidly during platelet contraction to be reliably tracked over time. To prevent bead mismatch from influencing results, we checked bead displacement maps prior to traction force calculation and performed manual corrections where necessary (figure IV.5 in section IV.2). The other drawbacks of particle tracking mentioned earlier could not be prevented from affecting the spatial resolution of our experiments. These notwithstanding, we achieved a very satisfactory resolution of $0.2-0.3 \mu \mathrm{m}$ in our measured traction force fields (section IV.6).

During bead traction we encountered yet another difficulty: platelets, once activated, secrete attractants for other platelets (section I.1.2, p. 14). Hence, we noticed that in the vicinity of some platelets there were neighboring cells that were also tugging on the substrate. We masked out beads, where the interference of these neighbors was most noticeable in the bead displacement maps (figure IV.5 in section IV.2). However, the remaining beads must necessarily show reduced displacements under the counteracting influence of the neighboring cells. Consequently, traction forces for some platelets are slightly underestimated.

Further errors in traction forces arise from the force calculation procedure itself. We have seen earlier (section I.4) that solving equation I.1 to obtain traction forces is not straightforward. Equation [.1] describes an ill-posed problem, meaning that small experimental errors can lead to great variations in calculated traction forces. To solve this problem, we have used a regularization term in agreement with the approach by Dembo and Wang [11. This regularization favors simple solutions for the traction force fields (section IV.4) and accordingly, the morphology of calculated traction force fields may be a little influenced by regularization. Furthermore, traction force calculation was restricted to the cell area $A$. Physiologically this makes sense, as the cell area is the only region where force transduction to the substrate can occur. Nevertheless, it was noted by Butler et al. [7] that with such a restriction, traction forces at the edge of cells tend to be overestimated.

Finally, the Boussinesq approximation in equation I.1 may potentially also introduce inaccuracies to calculated force fields. As Dembo and Wang [1] and Butler et al. [7] have pointed out, the approximation is applicable to cases, where the displacements and 
the lateral dimensions of both the cell and the region of noticeable bead displacements are small compared with the substrate thickness. These conditions are not fully met in our case. While the maximum displacement $\left(\sim 0.9 \mu \mathrm{m}\left[{ }^{[1]}\right)\right.$ and the cell diameter $\left(\sim 2-4 \mu \mathrm{m}{ }^{[2]}\right)$ are typically much smaller than the substrate thickness $(53.3-104.3 \mu \mathrm{m}$ measured at our microscope), the region of noticeable displacements is, while still smaller, on the same order of magnitude $(\sim 15 \mu \mathrm{m} 1])$. Fortunately, the LIBTRC program that we use to calculate traction force fields makes a correction for the finite thickness of substrates $[3]$. We therefore conclude that Boussinesq's approximation introduces no further, significant inaccuracies to our data.

In summary, we find that total forces in our experiments are slightly, but systematically underestimated (by about $13 \%$ ) and traction forces at the cell edge may be slightly overestimated. In interpreting our data, we therefore need to take this small bias into account. Considering the difficulties both of the experimental technique and the data analysis, our results are nevertheless satisfactory. Having detailed errors in our measurements, we now proceed to the interpretation of our results.

\section{VI.2 Platelet Contractile Forces}

Using TFM as an experimental technique we measure on average total, steady state contractile forces per platelet of $34 \mathrm{nN}$. Compared to previous reports (section I.3), we find that our result agrees well with a single platelet AFM study by Lam et al. [28], which yielded a platelet force of $29 \mathrm{nN}$. However, if we compare our results with previous studies on cell aggregates, we notice a discrepancy of at least one order of magnitude. For example, Jen and McIntire [25] enclosed clotting platelet rich plasma samples in between two plates and measured the forces that the clots exerted on the plates. From these measurements they extrapolated a platelet force of $\sim 0.5 \mathrm{nN}$. Another study by Liang et al. 33] using smaller cell ensembles, in which microclots were observed on elastic microposts, still yielded forces per platelet of $2.1 \mathrm{nN}$. The marked difference between these studies and our own findings is all the more striking, considering that in our results total platelet forces tend to be underestimated (see previous section VI.1).

One possible explanation for the deviating results lies in the fact that the aforementioned experiments on cell aggregates probe contraction only along selected directions. As our measurements show, however, platelet contraction is nearly isotropic. Thus, all contractile force contributions from the directions that are not measured on cell aggregates are also lacking in the final platelet force. Possibly this is also the reason why our single cell experiments display slightly larger forces than Lam et al.'s, which are restricted to uniaxial contraction. Yet, considering that the difference between our result and Lam et al.'s is small compared to the differences between single cell and cell aggregate measurements, it seems more likely that sample size plays the more important role in explaining the deviating results. That is, for cell aggregates the platelet force is extrapolated from the total contractile force of the aggregate under the assump-

\footnotetext{
${ }^{1}$ according to the bead displacement field of a typical platelet (figure IV.5

2 This corresponds to twice the typical cell radius $R$ (see section IV.7.2.

${ }^{3}$ Due to the fact that the correction term is as yet unpublished, the LIBTRC manual does not provide further details as to the corrections made.
} 
tion that platelet forces add up linearly. Put a little differently, it is assumed that force transduction across inter-platelet connections occurs without loss, as if the connections were infinitely rigid rods. In reality, however, platelets are interconnected by flexible fibrin strands (section I.1.2, p. 15). Platelet forces will therefore not add up linearly, but rather a portion of the forces will be lost to deformation of the fibrin links. The larger the platelet aggregate the more noticeable will be the loss of forces to the fibrin network, and hence the smaller will be the extrapolated platelet force. Interestingly, platelets send out biochemical signals to other platelets, which may in turn influence platelet force generation in a clot. However, as indicated in section I.1.2 (p. 15), these signals rather seem to stabilize platelets than to reinforce platelet contraction. Here, future experiments will hopefully provide deeper insights into platelet-platelet interactions and how these may affect measured platelet forces.

\section{VI.3 Platelet Force Generators}

We have seen in section V.1.1 that platelets exhibit high traction forces of $\sim 3.3 \mathrm{kPa}$. Compared with traction forces of $0.8-3.03 \mathrm{kPa}$ for fibroblasts [11, 41, 49], or traction forces on the order of $0.1 \mathrm{kPa}$ for both airway smooth muscle cells [7, 55] and fish keratocytes [44], platelets are among the strongest cells measured by TFM. Yet, how are platelets able to generate such large traction forces? To address this question, we subsequently consider different mechanisms of force generation and discuss their possible contributions to measured traction forces.

\section{Traction force contribution of acto-myosin}

The main force generator during platelet contraction is generally believed to be myosin in association with actin ([38, p. 88], [16, 31]). Whether myosin is able to generate the bulk of the average total platelet force that we measure, can be appraised by a simple calculation. A platelet contains on average 12,000 non-muscle myosin II motors [38, p. 78]. Each of these motors can generate a force $F_{m, s}$ of $1.3-3.5 \mathrm{pN}$ [56, 40, 17, 20] (see also section I.2), leading to a total force $F_{m, t o t}$ of $15.6-42 \mathrm{nN}$. Our measured average total platelet force is $34 \mathrm{nN}$, which is well within the range of $15.6-42 \mathrm{nN}$ that platelet myosin can generate. That is, the $34 \mathrm{nN}$ are within the capacity of myosin motors, if all motors would act synchronously. However, we have seen in section I.2 that the most prominent platelet myosin isoforms, non-muscle myosin IIA and IIB, are non-processive motor proteins with a duty ratio of $0.05-0.11$ [27] or 0.23-0.4 [57], respectively. As a consequence, only about 5-11\% of all myosin IIA heads and only about $23-40 \%$ of all myosin IIB heads bind actin simultaneously at each time point. The effective total

myosin force $F_{m, t o t}^{\text {eff }}$ is therefore smaller than our initial estimate $F_{m, t o t}$. For a precise number on the $F_{m, t o t}^{\text {eff }}$, we need information on the relative abundance of myosin IIA and myosin IIB in platelets. Unfortunately, this information is not readily available from the literature. In an immunofluorescence study by Maupin et al. [36] it is mentioned that staining of non-muscle myosin IIA in platelets shows clearly under the microscope, while staining of the IIB isoform is hardly visible at all. This indicates at least that myosin IIB content in platelets is low. We therefore approximate the 12,000 myosin 
motors in platelets to be all non-muscle myosin IIA. According to the duty ratio of non-muscle myosin IIA and by considering that myosin has two heads, we deduce that only about $1200-2640$ of the platelet myosin IIA motors bind simultaneously to actin. Hence, we obtain an effective myosin total force $F_{m, t o t}^{\text {eff }}$ of $1.56-9.24 \mathrm{nN}$. It should be noted that the value for $F_{m, s}$ that we used to estimate $F_{m, t o t}^{\text {eff }}$ is only an average. For individual myosin motors, forces as high as $7 \mathrm{pN}$ have been measured for $F_{m, s}$ [17]. This leads to an upper limit for $F_{m, t o t}^{\text {eff }}$ of $18.48 \mathrm{nN}$. Still both the upper limit and the average value for $F_{m, t o t}^{\text {eff }}$ are underestimates for two main reasons. On the one hand, the presence of a few platelet myosin IIB motors leads to an increase in $F_{m, t o t}^{\text {eff }}$. On the other hand, regulatory signals in platelets may enhance myosin binding to actin so that more motors act simultaneously to contract the actin bundles [24]. All points considered, it seems realistic to assume that the main contribution in our measured forces is provided by myosin contraction. Corroborating this idea is the fact that it takes platelets roughly as long to generate maximum traction forces (25 min, section V.1.1) as it takes actin to reorganize into a network capable of efficiently transmitting myosin forces $(\sim 30 \mathrm{~min}$, section V.1.2).

Nevertheless, additional force contributions are needed to explain the total forces that we measure. Most of these will be passive, elastic force contributions, which we will discuss in more detail in the following section. However, we cannot exclude that other force generating mechanisms, such as actin bundle contraction via actin depolymerization, play a role (see section I.2). In fact, Farrell et al. [15] have measured actin polymerization/depolymerization to generate forces of 4 to $5 \mathrm{pN}$ per actin filament. An estimation of how these forces contribute to the total platelet force won't be pursued here, as we are still lacking information on F-actin content for platelets activated on PAA.

\section{Elastic contribution to traction forces}

The traction forces we measure with TFM during platelet activation will have contributions from passive elastic stresses. These elastic stresses form as a response to platelet spreading to counteract the shape changes that the platelet undergoes (see also sections I.2 and II.2). In fact, Feghhi and Sniadecki [16] have demonstrated in their study of clots on elastic pillars that after inhibition with blebbistatin of the platelets active force generator, myosin, the clots still retain about half of their contractile forces. The remaining forces are likely elastic in nature and of these elastic forces again only a part will be contributed to by platelets. The rest of the elastic force contribution will be provided by the inter-platelet linkers in the clot, the fibrin fibers. To estimate the contribution of elastic stress for single platelets, we therefore resort once more to section II.1.1 and to equation II.15 for the stress-strain relationship in isotropic, elastic objects:

$$
\sigma_{i j}=\frac{E}{1+\nu}\left(\epsilon_{i j}+\frac{\nu}{1-2 \nu} \delta_{i j} \sum_{k} \epsilon_{k k}\right)
$$

where $\sigma_{i j}$ is the stress, $\epsilon_{i j}$ is the strain, $E$ is the Young's modulus of the object and $\nu$ is the Poisson ratio of the object. To apply the above equation to platelets, we need to make a few approximations. For simplicity, we first approximate the rounded shape 
at the beginning of cell spreading by a cube. We assume that this rounded shape corresponds to the unstressed shape of the cell, once the microtubule coil is dismantled or compressed to the cell center upon platelet activation $\left[{ }^{[4]}\right.$ (see also section I.1.2). To further facilitate calculation, we describe cell spreading by considering a homogeneous dilation of the cell/cube in the $x_{1}-x_{2}$-plane with $\sigma_{11}=\sigma_{22}=: \sigma$ and $\sigma_{33}=0$. With these assumptions, we can then use the above equation and substitute the conditions for $\sigma_{11}, \sigma_{22}$ and $\sigma_{33}$ for $\sigma_{i j}$. We thus obtain after some calculation:

$$
\epsilon_{11}=\epsilon_{22}=: \epsilon ; \quad \epsilon_{33}=\frac{-2 \nu}{1-\nu} \epsilon
$$

and in particular:

$$
\sigma=\frac{E}{1-\nu} \epsilon
$$

With expression VI.1, we can now insert appropriate values for $E, \nu$ and $\epsilon$ to obtain a rough estimate for the elastic stress $\sigma$ that is generated by single platelets.

From section $I .2$ we can take $E$ and $\nu$ to be $4 \mathrm{kPa}$ and 0.5 , respectively. To estimate the strain $\epsilon$ we consider the example cell that is shown in section V.1.1 (figure V.2 a) on p. 83 and inset in figure V.3 a) on p. 84). We choose this cell in particular, because its steady state total force $F_{t o t}^{S t}=39.7 \mathrm{nN}$ according to figure $\mathrm{V} .2$ a) is close to our ensemble average of $34 \mathrm{nN}$. Likewise its steady state average traction force $T_{A}^{S t}=3.6 \mathrm{kPa}$ is close to our ensemble average of $3.3 \mathrm{kPa}$. To estimate $\epsilon$ we go back to our cubeapproximation of the cell. We set the area of a square face of the cube to the measured cell-substrate contact area $A$. According to the inset in figure V.3 a) this means that the cube has a face area of $A_{0} \approx 9.86 \mu \mathrm{m}^{2}$ in its unstressed state, that is, at the beginning of platelet spreading. Here, we obtain $A_{0}$ by averaging the time track in figure V.3 a) for all time points before the cell reaches its steady state $\left(t<t_{t p}\right)$. As soon as the cell reaches its steady state $\left(t>t_{t p}\right)$ and is fully spread, we measure an average cell / cube face area of $\bar{A} \approx 12.87 \mu \mathrm{m}^{2}$. Hence, the side length $a$ of the cube is stretched during spreading by an amount $\delta a=(\sqrt{12.87}-\sqrt{9.86}) \mu \mathrm{m}$. The strain is accordingly $\epsilon \approx \delta a / a_{0} \approx(\sqrt{12.87}-\sqrt{9.86}) / \sqrt{9.86}$.

With this value for the strain $\epsilon$ and the previously mentioned values for the Young's modulus $E=4 \mathrm{kPa}$ and the Poisson ratio $\nu=0.5$, we can finally estimate with equation VI.1 the elastic stress for single platelets to be $\sigma \approx 1.14 \mathrm{kPa}$. That is, roughly a third of $T_{A}^{S t}$ is contributed to by passive elastic forces. Seeing that $F_{\text {tot }}^{S t}$ and $T_{A}^{S t}$ are related by a multiplication with the cell area $A$ (see section IV.4), the same holds true for $F_{t o t}^{S t}$. Of course, our estimate for the elastic stress is only very rough. Technically, for example, the cell is not an isotropic object. Then again, isotropy is also assumed in the measurement of the platelet Young's modulus $E$ [48], meaning that we are already using an effective rather than an actual Young's modulus E. Platelet spreading, however, is also not homogeneous: the cell extends thin filopodia and lamellipodia (see also the left hand side of figure IV.13, p. 80. This inhomogeneous spreading of platelets can cause elastic stresses to be locally higher, yet the average may even be smaller than

\footnotetext{
4 The discoid shape of the resting platelet is maintained only because of the microtubule coil running the long circumference of the platelet. In the absence of the microtubule coil platelets have a spheroid resting shape [38, p. 90].
} 
our estimate. Finally, platelets are not cubic in shape, although it is unclear which shape is best used to model the unstressed state of the platelet. As we have mentioned earlier a spherical form may be more appropriate than the discoid shape observed for resting platelets. The reason for this being that the discoid shape is maintained only by the microtubule coil, which in turn gets disassembled or compressed during platelet activation. However, the different stages during platelet activation overlap, so that the microtubule coil is probably not fully rearranged before the platelet starts spreading. Hence, the initial rounding of the platelet at the beginning of activation likely does not produce a perfectly spherical, unstressed shape. All arguments withstanding, our estimate provides a good first guess on the elastic stresses generated by single platelets.

\section{VI.4 Mechanical Models of Platelet Contraction}

In addition to characterizing platelet forces, we have described the spatial distribution of actin, myosin and vinculin during platelet contraction on soft PAA substrates in chapter $\mathrm{V}$ (section V.2.2). We have thus observed that the bulk of actin is found at the cell center and that it is surrounded by a ring-like structure of myosin motors. We have furthermore noticed that the focal adhesion protein vinculin is located almost exclusively at the cell periphery. These two findings suggest that force generation by acto-myosin contraction occurs primarily in the cell body, while generated forces are transmitted to the environment via focal adhesions mostly at the edge of the cell. Hence, we see the larger traction forces at the edge of the cell during our force measurements (figure V.1. p. 81). Another interesting point about platelet force generation is that they generate isotropic fields. The images taken of actin, vinculin and myosin provide us with two reasons why this is so. Firstly, the ring-like structure of myosin enables the cells to generate forces isotropically. Secondly, vinculin, and therefore focal adhesions, are distributed throughout the cell periphery, thus allowing for force transduction also to proceed isotropically. These observations affect our understanding of platelet activation. Contrary to earlier assumptions suggested by experiments of platelet activation on glass [54] (see also section I.3), on soft substrates myosin is not evenly distributed along actin. We find that on PAA substrates the bulk of actin and myosin form separate, albeit overlapping structures (figure V.9, p. 92). The inhomogeneous distribution of myosin along actin, in turn, has implications for any future model for platelet contraction.

\section{Network models}

Let us consider, for example, the models presented by Guthardt Torres et al. [22], in which the actin cytoskeleton in a cell is approximated by a network of mechanical links (see also section II.2.1). These links are taken to be either Hookean springs, passive cables or actively contracting cables. The latter case of actively contracting cables is, in fact, an extension of passive cables in that it includes contractile elements (myosin motors) that are associated with the cables. For the active cable network (ACN), in particular, it is assumed that myosin motors distribute evenly along actin bundles. To apply an ACN model to platelet contraction, we would therefore need to adjust the 
model. By considering, for example, two types of active cables with different densities of associated contractile elements / myosin motors, we might be able to account for the inhomogeneous distribution of myosin along platelet actin. Whatever the network model for platelets, the network geometry would have to be adjusted. While Guthardt et al. primarily considered regular networks, we need to choose a network which is denser at the cell center to mimic the bulk of actin that we see at the center of platelets. The adhesion geometry, in turn, also has to be adjusted to include more adhesion sites forming a ring-like pattern at the cell periphery (see for example figure V.9, p. 92).

When deciding which of the network models is the more appropriate one for describing platelet contraction, we find that our experimental evidence points toward an ACN model. As shown in the previous section VI.3, myosin contraction probably generates about $2 / 3$ of the total platelet force and the ACN model is the only model to include myosin contraction. In addition, we have seen that platelet contraction is nearly isotropic despite some irregularity in the platelet shapes or focal adhesion geometries due, for example, to filopod formation (figure V.9. p. 92). This indicates that force generation in platelets is, to some degree, independent of the precise distribution of focal adhesions. To use the technical terms of sectionII.2.1, platelet contraction seems to respond locally rather than globally to focal adhesion geometry. Such a local response is a characteristic of the ACN model. In Hookean spring networks (HSN) or passive cable networks (PCN), by contrast, responses are global, meaning that the influence of the adhesion geometry on network deformation and force distribution reaches far into the network.

There are, however, also arguments against using an ACN model for describing platelet contraction. Favoring these counter-arguments is the fact that platelets do not show the strong localization of forces to the edge of the cell which are so typical for ACNs. That is, though there are elevated traction forces at the cell periphery (figure V.1. p. 81), they do not differ by orders of magnitude from the traction forces in the bulk of platelets. Furthermore, it is known that forces at the cell edge tend to be slightly overestimated by TFM due to artifacts resulting from data analysis (see section I.4 and VI.1). Taking the force distribution at the cell edge into account, both HSNs and PCNs describe more accurately our observed gradual decrease of traction forces from the cell edge to the cell center (figure V.1. p. 81). Nonetheless, it is as yet unclear how our proposed changes for the network geometry will affect the force distribution in the different network models. The ACN is therefore still the most promising of the three network models for describing platelet contraction.

Finally, we would like to point out that the ACN network model, or any of the considered network models for that purpose, can only ever represent a time average of platelet contraction in its final state. In reality, however, the acto-myosin structures that are formed after 30 min of platelet activation (figure V.4, p. 86) are not static. As demonstrated by the highly dynamic platelet shape throughout our TFM movies (figure V.1. p. 81; see also section VII), part of the platelet actin at least undergoes constant assembly and disassembly. 


\section{Models for stress fiber polarization}

To characterize the mechanisms leading to the development or redevelopment $\left[{ }^{[5]}\right.$ of the acto-myosin network in activated platelets, we turn to another model presented in section II.2.2. This model by Zemel et al. [65] considers how elastic stresses that accompany cell spreading can induce the formation or alignment of cell-internal force dipoles. In this case, the internal force dipoles are related to acto-myosin subunits. It should be noted, however, that a single force dipole is not an exact representation of an acto-myosin subunit. Therefore, it is of no great consequence to the model if myosin is not evenly distributed along actin. The activity of myosin clusters is simply projected along the entire length of the associated actin filaments and an effective force dipole moment is obtained for the filament subunits. In their model Zemel et al. assume the cell to be initially homogeneous and isotropic, while both the cell shape and the initial cell spreading behavior are taken to be asymmetric. Preferred orientations of the internal force dipoles are furthermore interpreted as stress fiber formation.

To discuss the applicability of Zemel et al.'s model to platelet activation, we first consider their initial assumptions. We have seen in section I.1.1 that platelets are never really homogeneous or isotropic, yet they can be approximated to be so on the basis of our F-actin staining that looks rather homogeneous at the beginning of platelet activation (figure V.4, p. 86). Furthermore, Zemel et al. consider a volume-averaged dipole moment density $\left\langle p_{i j}\right\rangle_{V}$ so that small heterogeneities are averaged out. For the initial cell shape, in turn, we find that at the beginning of activation platelets round up approximating a symmetric shape (see figure I.5 on page 10 or figure IV.13 on 80, without ever truly becoming symmetric due to filopod formation. Yet, any asymmetry causing stress fiber polarization in platelets must originate mainly from asymmetric spreading. On PAA substrates, however, it is difficult to assess asymmetric spreading, because of the very dynamic cell outline. We observe local extensions and retractions of filopodia and lamellipodia, which are, for example, reflected in the variations in cell area that are seen in the inset of figure V.3 a) (p. 84). These move constantly and seldom persist over time. Accordingly, we also see less distinctive stress fibers for platelets on PAA and no prevailing orientation in the actin network (figure V.4. p. 86). On glass surfaces, the scenario is clearer. Here we observe that some platelets do spread asymmetrically (see figure I.5, p. 10). Hence, we also detect some preferred orientations in stress fiber patterns for platelets on glass (figure V.4, p. 86). The fact that we observe stress fiber polarization only on hard surfaces, such as glass, reinforces the idea that stress fiber orientation in platelets is influenced only by an initial asymmetry in spreading behavior. After all, Zemel et al. have shown that spreading asymmetry supplies stress fiber polarization with a contribution that monotonically increases with substrate stiffness. To further corroborate this idea, we intend to vary the substrate stiffness in future experiments and study platelet contraction as a function

\footnotetext{
${ }^{5}$ In section I.1.1 p. 2 it is shown that the resting platelet already has an existing, rudimentary acto-myosin network containing about half of the total platelet actin content. The remaining actin is present in the resting platelet in the monomeric phase. During platelet activation therefore two processes contribute to the observed acto-myosin network formation. One is a reorganization of the existing acto-myosin network in the resting platelet and the other is the formation of new filaments from the pool of monomeric actin.
} 
of substrate elasticity. Here, we have an additional advantage as we can use the force dipole moments $D_{ \pm}$already calculated for the major and minor contractile axis in our TFM data sets to determine the degree of polarization in the cell. By these means we can generate a plot similar to the ones shown in section II.2.2 (figure II.5, p. 46) and compare our findings directly with predictions from Zemel et al.'s model. Thus, we further hope to contribute to the discussion about possible cytoskeletal polarization mechanisms. In particular, we are interested in finding out whether our data correspond to the limit of either axially induced polarization or purely orientational polarization. The first limit implies that stress fiber assembly and disassembly in perpendicular directions is fully decoupled. The second limit, in turn, assumes that the amount of cell-internal force dipoles remains constant, so that stress fiber formation in one direction leads to a disassembly of stress fibers in the perpendicular direction.

\section{Final remarks on modeling}

There is a final point in our data that no model presented so far can explain, namely the concentration of myosin at some points along actin filaments during platelet activation on PAA. In particular, we refer to the observed formation of a myosin ring-like structure surrounding the bulk of actin in the platelet center (figure V.9, p. 92). This phenomenon is very likely connected to the fact that actin network development is in its early stages strongly localized. Thus, initially, actin polymerization occurs predominantly at the cell periphery (figure V.4 p. 86). This, in turn, is what we expect considering the chemical signaling at the platelet membrane that regulates actin polymerization (section I.1.2, p. 7). However, it would be interesting to see how myosin interacts with actin throughout the cytoskeletal reorganization process. Do we see a myosin ring because there is a particular time point during actin polymerization at which myosin begins to associate with actin? How is the situation different for platelet activation on glass? Future experiments on myosin staining will hopefully shed some light on these points.

\section{VI.5 Platelets as Model Systems for Cellular Contraction}

With the results from the previous chapter $\mathrm{V}$ we can now refer back to section $I .2$ and see whether platelet contraction is representative of cellular contraction in general. Let us consider, for example, the role of myosin II activity during cellular contraction. In section I.2 we have seen that different cellular systems all use myosin II activity to generate active contractile forces. Platelets are no exception to the rule. In confirmation of the prevailing opinion in the community ([38, p. 88], [16, 31]), we find that the active contribution to our force measurements on platelets can be satisfactorily explained by myosin II motor contraction (section VI.3).

This is where the likeness in characteristics shared by all cells generating contractile forces comes to an end. Nevertheless, we perceive similarities between platelets and members of subgroups of the aforementioned cells. To examine these similarities and also point out marked differences, we will focus on the questions that have been raised 
in section I.2. In particular, we will analyze aspects of speed, directionality and flexibility during contraction. With regard to contraction speed, we have already seen that it takes platelets about 25 min to establish maximum forces. This time delay is primarily caused by the fact that the acto-myosin network in platelets still has to reorganize into a fully functional, contractile network (see also section VI.3). Motor efficiency, which is significant for the speed of action of skeletal and smooth muscle cells (section I.2), has only a negligible influence on the delay time during platelet contraction. The loose ordering of acto-myosin structures in platelets (figure V.9, p. 92) will likewise add little to the overall delay. In their loose ordering of acto-myosin, platelets resemble smooth muscle cells and locomoting cells, although macroscopically acto-myosin patterns can differ visibly from cell type to cell type. For example, in fibroblasts, fish keratocytes and smooth muscle cells, actin and myosin have preferred orientations (section I.2 and reference [21]). By contrast, in platelets they form isotropic structures. Consequently, traction force fields for the first three cell types are polarized [41, 44, 55, whereas platelet forces are again isotropic. Considering the different functions of the cells, it makes sense that their generated force fields should be morphologically different. Fibroblasts, fish keratocytes and smooth muscle cells all have a clear direction of motion or action, hence the polarized force fields. Platelets, in turn, need to homogeneously reduce clot size during clot retraction, which they achieve by pulling on their intercellular links from all directions. The isotropy in platelet force fields is, perhaps, the feature that most distinguishes platelet contraction from other cellular contraction. In all other respects, there is a semblance between platelets and some other cell types undergoing contraction. This also holds true for the aspect of flexibility during contraction. As noted before in section I.2 in motile cells contractile acto-myosin structures are assembled as the need arises in order to grant maximal flexibility of cell motion. Similarly, in platelets acto-myosin only assembles into a maximally efficient, contractile network if platelets have been activated. By these means our organism preserves maximal flexibility for a prompt response to injury, while preventing accidental clot formation under normal blood flow conditions. 


\section{Summary and Outlook}

The objective of this study has been to look into the phenomenon of cellular contraction by both measuring forces and staining cellular components involved in force generation or force transduction, using the example of human blood platelets. We successfully adapted the experimental technique, traction force microscopy (TFM), to meet two challenges: the small size of platelets and the extended temporal record of traction forces during platelet contraction. By adapting TFM we were able to record, for single platelets, traction force fields with both spatial as well as temporal resolution. Furthermore, we measured traction forces on soft polyacrylamide (PAA) substrates with an elasticity in the physiologically relevant range $(4 \mathrm{kPa})$. So far, studies on platelet forces have commonly been performed on unphysiologically hard substrates such as glass [28. Similarly, the staining of platelet components has traditionally been carried out on hard glass cover slips [54]. Here, too, we introduced a novelty by staining both platelet acto-myosin, as well as the focal adhesion protein vinculin during platelet contraction on soft PAA substrates. As platelets cannot be transfected to fluoresce by standard techniques, we chose an indirect method by fixing platelets prior to staining so that these experiments were performed separately from force measurements with TFM.

Our results show that it takes platelets about 25 min on PAA to organize their contractile system, acto-myosin, into an efficient force generating network. In this final network, myosin is not evenly distributed along actin, contrary to what is generally believed based on experiments on glass. Also, acto-myosin forms less distinctive and less ordered stress fibers on PAA as compared to glass. Instead, the acto-myosin network takes on an isotropic appearance with the bulk of actin at the platelet center being surrounded by a myosin ring. Due to this isotropic organization, the platelet contractile system is able to generate nearly isotropic forces which point toward the cell center and are transmitted to the extracellular environment through focal adhesions primarily at the edge of the cell. Acto-myosin contraction is assisted by passive elastic forces arising from cell shape changes during platelet spreading on the substrate. Possibly, actin depolymerization contributes further to force generation resulting in final total platelet forces of $\sim 34 \mathrm{nN}$, or average traction forces of $3.3 \mathrm{kPa}$. These forces, measured for single platelets, are considerably larger than estimated platelet forces from experiments on platelet aggregates [25, 33], which demonstrates the importance of the single cell approach. In comparison with other cells generating contractile forces, platelets are set apart by the isotropy in acto-myosin structures and the resulting isotropy in generated forces.

In order to apply a mechanical network model [22] to platelet contraction, it is therefore necessary to account for the particular distribution of actin and myosin in the cells. 
A comparison with a theoretical model for stress fiber formation [65], in turn, indicates that stress fiber alignment on glass is driven by an asymmetry in platelet spreading.

Although we have shed light on quite a few points concerning platelet contraction, many more questions have been raised by our research, which we would like to address in future work. Most prominent among these questions is perhaps the question relating to how much other force generating mechanisms, apart from myosin contraction, contribute to our measured platelet forces. In section VI.3 we provided a rough estimate for the contri-

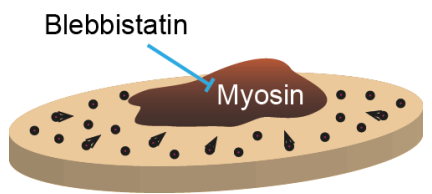

Figure VII.1: Measurement of myosinindependent force contributions during platelet contraction: In future experiments myosin activity will be blocked by addition of the drug blebbistatin in order to gain information on myosin-independent force contributions during platelet contraction. bution of, for example, passive, elastic forces using elasticity theory. It would be interesting to measure myosin-independent force contributions experimentally by inhibiting myosin during a TFM recording with the drug blebbistatin [26] (figure VII.1).

In addition to these experiments, we plan to apply one of the aforementioned mechanical network models to platelets to see whether we can theoretically reproduce our measured traction force fields. For yet another theoretical model on stress fiber formation, we would like to repeat TFM experiments, while varying the elasticity of the PAA substrates (figure VII.2, see also section VI.4). Here, we take advantage of the fact that for the polymer PAA, elasticity can be easily tuned by adjusting the monomer to cross-linker ratio (see also section I.4).

Another aspect that we would like to pursue further relates to the formation of the ring-like structure of myosin during platelet contraction on PAA. To find out more about this process, we would like to extend the time series on actin reorganization to include myosin staining patterns.

Tracing cellular contours in our TFM experiments, we noticed that the platelet shape remains highly dynamic even in the final stages of contraction (figure V.1). We ascribe this dynamic behavior to continuous, minor reorganization within the established actomyosin network of platelets. These reorganization steps are unfortunately undetectable in the snapshots taken of fixed platelets that were stained for actin and myosin. The lack of a nucleus in platelets furthermore renders it impossible to label actin in live human cells by genetic manipulation. Any conclusion drawn for the dynamics of the acto-myosin network will therefore have to originate from indirect observations of the network. As a first approach, we tried to access information on the network behavior by characterizing the movement of the cellular contours during our TFM experiments (figure VII.3). To that end, we picked a TFM data set, for which we had manually traced the cell contour obtaining a one-pixel-line $\left(x_{1, p x}(t), x_{2, p x}(t)\right)$ for each time frame $t$. We fitted a cubic spline function $\left(x_{1, s p}(s(t)), x_{2, s p}(s(t))\right)$ to the contour at each $t$, where the parameter $s$ was chosen to be the arc length along $\left(x_{1, p x}(t), x_{2, p x}(t)\right)$ in clockwise direction. As a starting point for the arc length $(s=0)$, we always set a 

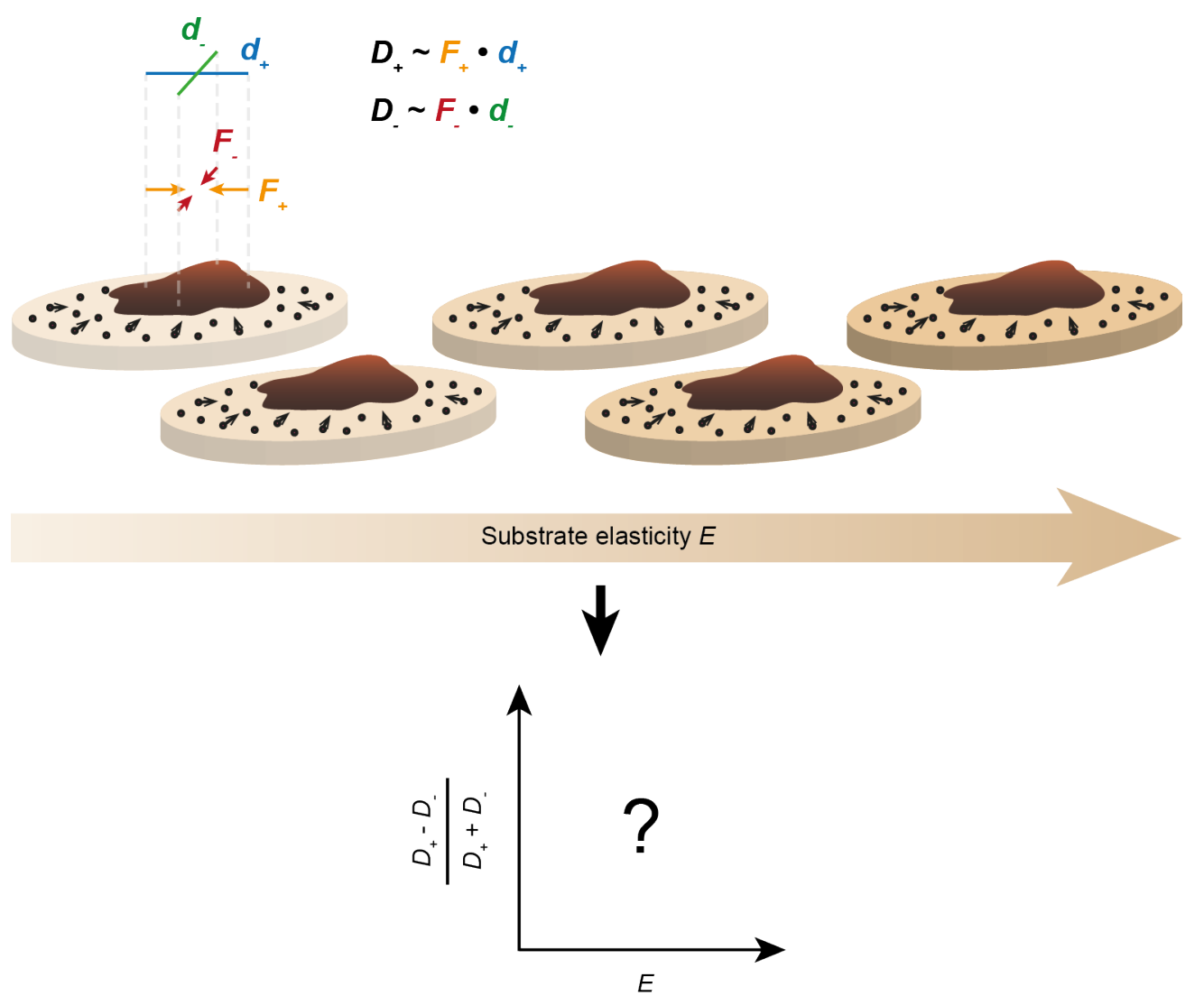

Figure VII.2: Observation of stress fiber formation during TFM experiments on substrates with varying elasticities: To test a theoretical model on stress fiber formation, we plan to repeat TFM experiments on substrates of different stiffnesses and observe the influence of substrate elasticity $E$ on the polarization of platelet force fields. To evaluate the degree of polarization, we will use, as before, the force dipole moments $D_{+}$and $D_{-}$of the major and minor contractile axis of platelets (section V.2.1)

point on the contour (cyan arrow in figure VII.3) that was vertically above the center of the cell area (magenta cross in figure VII.3). We then calculated the curvature of the spline, $\kappa(s(t))=|\vec{\kappa}(s(t))|=\sqrt{\frac{\partial^{2} x_{1, s p}(s(t))}{\partial s(t)^{2}}+\frac{\partial^{2} x_{2, s p}(s(t))}{\partial s(t)^{2}}}$, and attributed a sign to $\kappa(s(t))$, depending on whether the vector $\vec{\kappa}(s(t))$ pointed toward the cell $(+)$ or away from the cell $(-)$. By color-coding $\kappa(s(t))$ for each point in a plot of $s / L$ vs. $t$, where $L$ is the cell perimeter, we obtained a so-called kymograph. In this kymograph, horizontal bright streaks are indicative of stationary filopodia, while diagonal bright streaks suggest filopod rotation along the cell circumference. Unfortunately, results from this preliminary approach were not conclusive and some features seen in the kymograph could not be traced back to any particular events in cellular movies. In the future, we would like to improve the quality of the kymograph by substituting $s$ for the more accurate arc length $s_{s p}$ of the spline fit, hoping thereby to reduce some of the noise seen in figure VII.3. Furthermore, we would like to generate a similar plot to the kymograph shown so far, where we encode the distance $d\left(s_{s p}(t)\right)$ of the contour to 


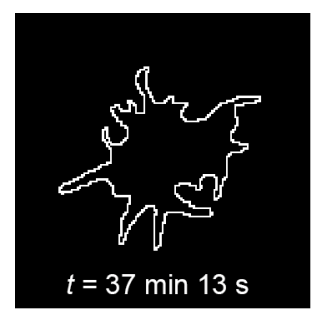

$1 \mathrm{px}$ line cell contour

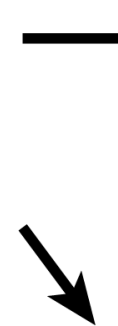

\section{Spline curvature} values $(\kappa)$ along

$1 \mathrm{px}$ cell contour line
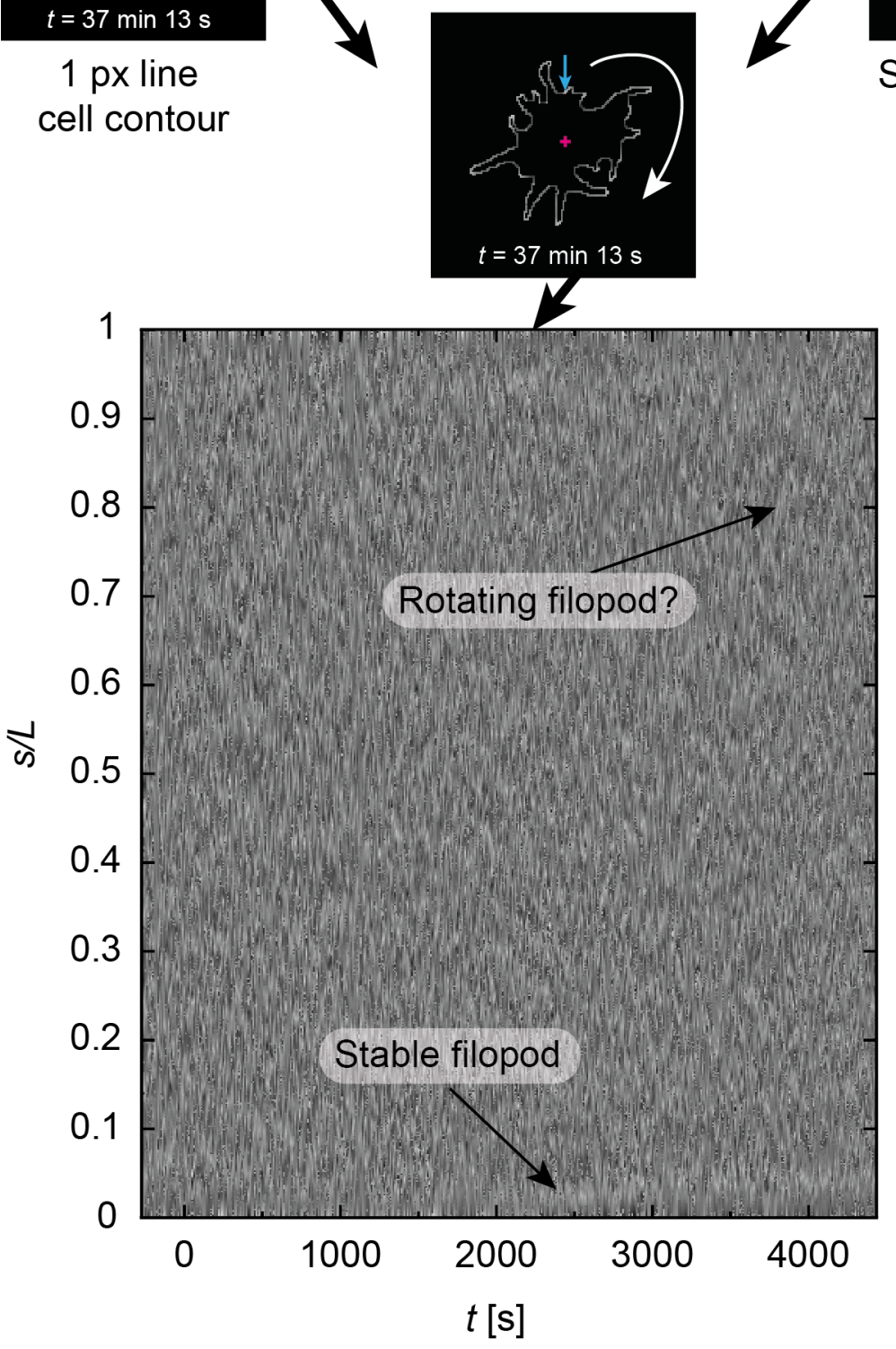

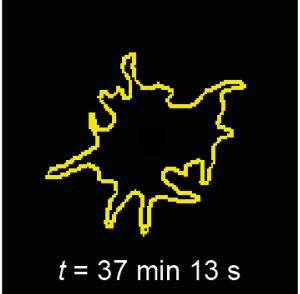

Spline approximation to cell contour

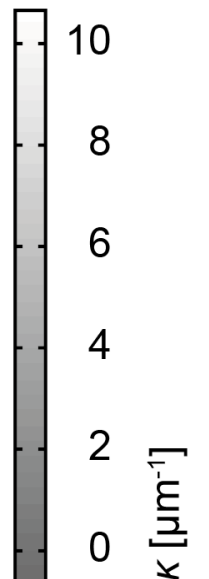

$-2$

$-4$

$-6$

$-8$

Figure VII.3: Preliminary kymograph to quantify the dynamic behavior of the platelet contour during contraction: For an example TFM data set, we fitted a cubic spline function (top right) to the cellular contour (top left) at every time $t$ and extracted the spline curvature $\kappa$ for each point on the contour (image at center). By running clockwise along the cell perimeter beginning at a point (cyan arrow) directly above the cell area's center (magenta cross), we collected values for $\kappa$ as a function of the cell contour arc length $s$. These were then color-coded in a plot of $s / L$ against $t$ (bottom image), where $L$ represents the total length of the cell perimeter. In this plot bright horizontal streaks represent filopodia that remain fixed over time, while bright diagonal streaks may be indicative of filopod motion along the cell contour. 
a)

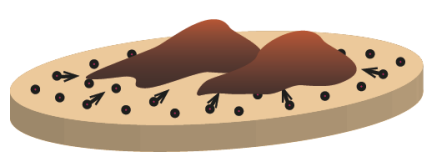

b)

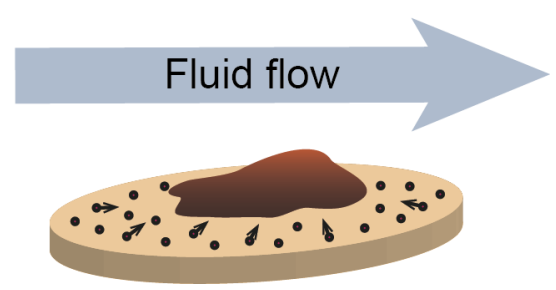

Figure VII.4: Approaching physiological conditions: a) We intend to take the next step toward measuring force fields in platelet clots by analyzing TFM recordings of pairs of interacting platelets. b) To simulate blood flow conditions during platelet contraction, we hope to be able to combine TFM with microfluidics.

the cell center instead of $\kappa$. In such a plot, the position of filopodia and lamellipodia should become more apparent.

Another attempt to indirectly observe cytoskeletal dynamics was more successful. As shown in section V.1.2, platelets take up beads that are added to the cell solution and transport them to the cell center during contraction. According to [32], these beads are incorporated into platelet granules so that we effectively see the process termed platelet internal contraction (section I.1.2, pp. 7), if we track these beads. Platelet internal contraction, in turn, is driven by acto-myosin. In the future, we would therefore like to follow up these preliminary experiments by a quantitative analysis of bead motion in contracting platelets.

All experiments described above are single cell experiments. By contrast, in medical studies, platelet aggregates are often measured in order to quickly obtain a statistically significant result. As we have seen earlier in this section, estimated platelet forces from aggregates do not correspond to platelet forces from single cell experiments. To bridge the difference in results and to add to our understanding of platelet-platelet interactions, we will in the future analyze TFM data already recorded for pairs of interconnected platelets (figure VII.4 a)) .

Finally, we hope to approach the physiological condition of platelet activation under blood flow by combining TFM with microfluidics (figure VII.4 b)). 



\section{Acknowledgements}

As is usual in the case of a research project like this one, progress and successful conclusion owe a great deal to the help, advice and support of other people, whom I would like to acknowledge here.

First among these is my supervisor, Prof. Sarah Köster, who has not only given me the opportunity to do research on such an interesting topic, but who has, in addition, always found the time to discuss both experimental intricacies and results. Not infrequently she has been ready to assist me in overcoming obstacles to my successful advancement that were not always directly related to my project as such.

It has furthermore been a pleasure to work in Prof. Köster's group, which I have found to be cooperative and very sociable. For the convivial moments spent with its members and for their professional support I am most grateful. Of these members, I would like, in particular, to acknowledge Rabea Sandmann, Susanne Bauch and Valeria Piazza. Both Rabea and Susanne were most helpful in assisting me in the preparation of my experiments. Valeria, in turn, has always been ready to share her expertise as far as biological aspects of my work were concerned, which was especially helpful with the staining procedures.

I am also much indebted to the following members of my thesis committee, Prof. Tim Salditt (Institute for X-Ray Physics) and Prof. Stephan Herminghaus (MPI for Dynamics and Self-Organization). Although it was not easy to organize our meetings, required by GGNB regulations, I very much appreciated their suggestions, which contributed considerably to the progress of my work. Furthermore, both have always shown a keen interest in my research, and our lively discussions in a friendly atmosphere turned each meeting into an agreeable affair.

Other fruitful suggestions came from Florian Rehfeldt of the Third Institute of Physics, with whom I also performed the rheology measurements to determine the elasticity of my substrates. Here, too, I had help from Christina Jayachandran of the same institute. For their assistance I am much obliged.

Earlier experiments to determine substrate elasticity with AFM turned unfortunately out to be of no avail. Nevertheless, my warmest thanks go to Constantin Spille of the Third Institute of Physics and Ingo Mey of the Institute for Organic and Biomolecular Chemistry for their readiness to perform the AFM experiments with me, for analyzing the recorded data and for sharing with us their reading of the result.

Most important to my work has been the collaboration we have established with the University Clinic of Göttingen. Not only have they provided us with the platelets, but the members of the medical staff there have also shown great and enthusiastic interest in our work, which has already led to one joint publication [53]. For their contributions to several fruitful discussions I am especially thankful to Joachim Riggert, Tobias Legler and Alexander Strate. 
While on the subject of collaborations, I would like to mention Hansjörg Schwertz, currently at the University of Utah (Salt Lake City, UT, USA), who has always been available for questions relating to the medical and biological aspects of my project. Additionally, he gave me the opportunity to visit Salt Lake City in the fall of 2009 to learn about platelet purification and staining protocols. His dedication throughout my visit and the warm welcome I received from him and his family, made the trip a memorable on. Thank you kindly for all.

Data analysis was facilitated by the program LIBTRC supplied by Micah Dembo (Boston University, MA, USA). For this and for his technical assistance I am much obliged.

Apart from the professional aid mentioned so far, I have received private support from both family and friends, which has been of equal importance to the success of my work. The members of my family, in particular, have encouraged and helped me throughout my studies, even offering expert answers to persistent doubts in Maths, English and Chemistry. I consider myself to be most fortunate in having such a great, knowledgeable and multi-faceted family. Thank you for being there; I wouldn't have achieved as much without you! Another person that has been invaluable to me is Jens. His encouragement has carried me through the more trying periods of my work. In addition, he has readily helped me by reading early drafts of my thesis and giving me technical advice on how to improve the images contained therein. Furthermore, he has kindly provided me with illustrative sketches for the microscopy techniques explained in section III.6.

Finally, I would like to acknowledge the financial support that my work has been given from the Deutsche Forschungsgemeinschaft (DFG) in the framework of SFB 937 and the Courant Research Centre "Nano-Spectroscopy and X-Ray Imaging", both of which were promoted by the Excellence Initiative. I have furthermore received a doctoral fellowship from the International Max Planck Research School for Physics of Biological and Complex Systems in Göttingen, for which I am much indebted. 


\section{Bibliography}

[1] D. A. Andrews and P. S. Low. Role of red blood cells in thrombosis. Current Opinion in Hematology, 6(2):76, 1999.

[2] J. D. Aplin and R. C. Hughes. Protein-derivatised glass coverslips for the study of cell-to-substratum adhesion. Analytical Biochemistry, 113(1):144-148, 1981.

[3] Y. Benveniste. A new approach to the application of Mori-Tanaka's theory in composite materials. Mechanics of Materials, 6(2):147-157, 1987.

[4] I. B. Bischofs, F. Klein, D. Lehnert, M. Bastmeyer, and U. S. Schwarz. Filamentous network mechanics and active contractility determine cell and tissue shape. Biophysical Journal, 95(7):3488-3496, 2008.

[5] B. K. Brandley and R. L. Schnaar. Covalent attachment of an Arg-Gly-Asp sequence peptide to derivatizable polyacrylamide surfaces: Support of fibroblast adhesion and long-term growth. Analytical Biochemistry, 172(1):270-278, 1988.

[6] W. Brown. Dynamic light scattering: the method and some applications, volume 49. Oxford University Press, USA, 1993.

[7] J. P. Butler, I. M. Tolić-Nørrelykke, B. Fabry, and J. J. Fredberg. Traction fields, moments, and strain energy that cells exert on their surroundings. American Journal of Physiology - Cell Physiology, 282(3):C595-C605, 2002.

[8] M. E. Carr Jr. Development of platelet contractile force as a research and clinical measure of platelet function. Cell Biochemistry and Biophysics, 38(1):55-78, 2003.

[9] M. E. Carr Jr and S. L. Zekert. Measurement of platelet-mediated force development during plasma clot formation. The American Journal of the Medical Sciences, 302(1):13-18, 1991.

[10] M. E. Carr Jr, A. Krischnaswami, and E. Martin. Method of using platelet contractile force and whole blood clot elastic modulus as clinical markers, 2007. US Patent 7,192,726.

[11] M. Dembo and Y.-L. Wang. Stresses at the cell-to-substrate interface during locomotion of fibroblasts. Biophysical Journal, 76(4):2307-2316, 1999.

[12] V. S. Deshpande, R. M. McMeeking, and A. G. Evans. A bio-chemo-mechanical model for cell contractility. Proceedings of the National Academy of Sciences, 103 (38):14015-14020, 2006. 
[13] E. K. Dimitriadis, F. Horkay, J. Maresca, B. Kachar, and R. S. Chadwick. Determination of elastic moduli of thin layers of soft material using the atomic force microscope. Biophysical Journal, 82(5):2798-2810, 2002.

[14] J. D. Eshelby. The determination of the elastic field of an ellipsoidal inclusion, and related problems. Proceedings of the Royal Society of London. Series A. Mathematical and Physical Sciences, 241(1226):376-396, 1957.

[15] B. Farrell, F. Qian, A. B. Kolomeisky, B. Anvari, and W. E. Brownell. Depolymerization of $\mathrm{F}$-actin produces a pulling force at the plasma membrane in vivo. Biophysical Journal, 98(3):19a, 2010.

[16] S. Feghhi and N. J. Sniadecki. Platelet contractile force assay using microposts and the role of nonmuscle myosin IIA regulation. 15th International Conference on Miniaturized Systems for Chemistry and Life Sciences (October 2-6, Seattle), 2011.

[17] J. T. Finer, R. M. Simmons, and J. A. Spudich. Single myosin molecule mechanics: piconewton forces and nanometre steps. Nature, 368(6467):113-119, 1994.

[18] M. J. Footer, J. W. J. Kerssemakers, J. A. Theriot, and M. Dogterom. Direct measurement of force generation by actin filament polymerization using an optical trap. Proceedings of the National Academy of Sciences, 104(7):2181-2186, 2007.

[19] N. Gavara and R. S. Chadwick. Determination of the elastic moduli of thin samples and adherent cells using conical atomic force microscope tips. Nature Nanotechnology, 7(11):733-736, 2012.

[20] W. H. Guilford, D. E. Dupuis, G. Kennedy, J. Wu, J. B. Patlak, and D. M. Warshaw. Smooth muscle and skeletal muscle myosins produce similar unitary forces and displacements in the laser trap. Biophysical Journal, 72(3):1006-1021, 1997.

[21] S. J. Gunst and D. D. Tang. The contractile apparatus and mechanical properties of airway smooth muscle. European Respiratory Journal, 15(3):600-616, 2000.

[22] P. Guthardt Torres, I. B. Bischofs, and U. S. Schwarz. Contractile network models for adherent cells. Physical Review E, 85(1):011913-1-13, 2012.

[23] L. D. Hall and J. C. Waterton. A method for determining the spatial distribution of spin-labeled organic ligands covalently bound to a noncrystalline surface: dipolar contribution to nitroxide EPR spectrum. Journal of the American Chemical Society, 101(13):3697-3698, 1979.

[24] M. E. Hemric, P. B. Tracy, and J. R. Haeberle. Caldesmon enhances the binding of myosin to the cytoskeleton during platelet activation. The Journal of Biological Chemistry, 269(6):4125-4128, 1994.

[25] C. J. Jen and L. V. McIntire. The structural properties and contractile force of a clot. Cell Motility, 2(5):445-455, 1982. 
[26] G. J. Johnson, L. A. Leis, M. D. Krumwiede, and J. G. White. The critical role of myosin IIA in platelet internal contraction. Journal of Thrombosis and Haemostasis, 5(7):1516-1529, 2007.

[27] M. Kovács, F. Wang, A. Hu, Y. Zhang, and J. R. Sellers. Functional divergence of human cytoplasmic myosin II. The Journal of Biological Chemistry, 278(40): 38132-38140, 2003.

[28] W. A. Lam, O. Chaudhuri, A. Crow, K. D. Webster, T.-D. Li, A. Kita, J. Huang, and D. A. Fletcher. Mechanics and contraction dynamics of single platelets and implications for clot stiffening. Nature Materials, 10(1):61-66, 2010.

[29] L. D. Landau and E. M. Lifschitz. Lehrbuch der Theoretischen Physik VII: Elastizitätstheorie. Akademie-Verlag Berlin (Germany), 6th edition, 1989.

[30] C. A. Lemmon and L. H. Romer. A predictive model of cell traction forces based on cell geometry. Biophysical Journal, 99(9):L78-L80, 2010.

[31] C. Léon, A. Eckly, B. Hechler, B. Aleil, M. Freund, C. Ravanat, M. Jourdain, C. Nonne, J. Weber, R. Tiedt, et al. Megakaryocyte-restricted MYH9 inactivation dramatically affects hemostasis while preserving platelet aggregation and secretion. Blood, 110(9):3183-3191, 2007.

[32] J. C. Lewis, J. E. Maldonado, and K. G. Mann. Phagocytosis in human platelets: localization of acid phosphatase-positive phagosomes following latex uptake. Blood, 47(5):833-840, 1976.

[33] X. M. Liang, S. J. Han, J.-A. Reems, D. Gao, and N. J. Sniadecki. Platelet retraction force measurements using flexible post force sensors. Lab on a Chip, 10 (8):991-998, 2010.

[34] H. Lodish, A. Berk, S. L. Zipursky, P. Matsudaira, D. Baltimore, and J. Darnell. Molecular Cell Biology. W. H. Freeman and Company, New York (USA), 4th edition, 1999.

[35] W. A. Marganski, M. Dembo, and Y.-L. Wang. [10] Measurements of cellgenerated deformations on flexible substrata using correlation-based optical flow. Methods in Enzymology, 361:197-211, 2003.

[36] P. Maupin, C. L. Phillips, R. S. Adelstein, and T. D. Pollard. Differential localization of myosin-II isozymes in human cultured cells and blood cells. Journal of Cell Science, 107(11):3077-3090, 1994.

[37] D. Meschede. Gerthsen Physik. Springer-Verlag Berlin Heidelberg (Germany), 23rd edition, 2006.

[38] A. D. Michelson. Platelets. Academic Press, Elsevier Science (USA), 2nd edition, 2006. 
[39] A. Mogilner and G. Oster. Force generation by actin polymerization II: the elastic ratchet and tethered filaments. Biophysical Journal, 84(3):1591-1605, 2003.

[40] J. E. Molloy, J. E. Burns, J. Kendrick-Jones, R. T. Tregear, and D. C. S. White. Movement and force produced by a single myosin head. Nature, 378(6553):209$212,1995$.

[41] S. Munevar, Y.-L. Wang, and M. Dembo. Traction force microscopy of migrating normal and H-ras transformed 3T3 fibroblasts. Biophysical Journal, 80(4):17441757, 2001.

[42] T. Mura. Micromechanics of defects in solids, volume 3. Springer, 1987.

[43] T. Oliver, K. Jacobson, and M. Dembo. [40] Design and use of substrata to measure traction forces exerted by cultured cells. Methods in Enzymology, 298: 497-521, 1998.

[44] T. Oliver, M. Dembo, and K. Jacobson. Separation of propulsive and adhesive traction stresses in locomoting keratocytes. The Journal of Cell Biology, 145(3): 589-604, 1999.

[45] C. S. Peskin, G. M. Odell, and G. F. Oster. Cellular motions and thermal fluctuations: the Brownian ratchet. Biophysical Journal, 65(1):316-324, 1993.

[46] G. Piazzesi, M. Reconditi, M. Linari, L. Lucii, P. Bianco, E. Brunello, V. Decostre, A. Stewart, D. B. Gore, T. C. Irving, et al. Skeletal muscle performance determined by modulation of number of myosin motors rather than motor force or stroke size. Cell, 131(4):784-795, 2007.

[47] T. Rabilloud. Mechanisms of protein silver staining in polyacrylamide gels: A 10-year synthesis. Electrophoresis, 11(10):785-794, 1990.

[48] M. Radmacher, M. Fritz, C. M. Kacher, J. P. Cleveland, and P. K. Hansma. Measuring the viscoelastic properties of human platelets with the atomic force microscope. Biophysical Journal, 70(1):556-567, 1996.

[49] P. Rajagopalan, W. A. Marganski, X. Q. Brown, and J. Y. Wong. Direct comparison of the spread area, contractility, and migration of balb/c 3T3 fibroblasts adhered to fibronectin-and RGD-modified substrata. Biophysical Journal, 87(4): 2818-2827, 2004.

[50] B. A. Roeder, K. Kokini, J. E. Sturgis, J. P. Robinson, and S. L. Voytik-Harbin. Tensile mechanical properties of three-dimensional type I collagen extracellular matrices with varied microstructure. Journal of Biomechanical Engineering Transactions of the ASME, 124:214-222, 2002.

[51] E. Sackmann and R. Merkel. Lehrbuch der Biophysik. Wiley-VCH Verlag GmbH \& Co., Weinheim (Germany), 1st edition, 2010. 
[52] U. S. Schwarz, N. Q. Balaban, D. Riveline, A. Bershadsky, B. Geiger, and S. A. Safran. Calculation of forces at focal adhesions from elastic substrate data: the effect of localized force and the need for regularization. Biophysical Journal, 83 (3):1380-1394, 2002.

[53] S. Schwarz Henriques, R. Sandmann, A. Strate, and S. Köster. Force field evolution during human blood platelet activation. Journal of Cell Science, 125:3914-3920, 2012.

[54] K. Tanaka and K. Itoh. Reorganization of stress fiber-like structures in spreading platelets during surface activation. Journal of Structural Biology, 124(1):13-41, 1998.

[55] I. M. Tolić-Nørrelykke and N. Wang. Traction in smooth muscle cells varies with cell spreading. Journal of Biomechanics, 38(7):1405-1412, 2005.

[56] M. J. Tyska, D. E. Dupuis, W. H. Guilford, J. B. Patlak, G. S. Waller, K. M. Trybus, D. M. Warshaw, and S. Lowey. Two heads of myosin are better than one for generating force and motion. Proceedings of the National Academy of Sciences, 96(8):4402-4407, 1999.

[57] F. Wang, M. Kovács, A. Hu, J. Limouze, E. V. Harvey, and J. R. Sellers. Kinetic mechanism of non-muscle myosin IIB. The Journal of Biological Chemistry, 278 (30):27439-27448, 2003.

[58] J. H.-C. Wang and J.-S. Lin. Cell traction force and measurement methods. Biomechanics and Modeling in Mechanobiology, 6(6):361-371, 2007.

[59] Y.-L. Wang and R. J. Pelham Jr. [39] Preparation of a flexible, porous polyacrylamide substrate for mechanical studies of cultured cells. Methods in Enzymology, 298:489-496, 1998.

[60] J. Weisel. Fibrin assembly. Lateral aggregation and the role of the two pairs of fibrinopeptides. Biophysical Journal, 50(6):1079-1093, 1986.

[61] D. Wessels, D. R. Soll, D. Knecht, W. F. Loomis, A. De Lozanne, and J. Spudich. Cell motility and chemotaxis in Dictyostelium amebae lacking myosin heavy chain. Developmental Biology, 128(1):164-177, 1988.

[62] J. G. White and S. M. Burris. Morphometry of platelet internal contraction. The American Journal of Pathology, 115(3):412-417, 1984.

[63] Z. Yang, J.-S. Lin, J. Chen, and J. H.-C. Wang. Determining substrate displacement and cell traction fields-a new approach. Journal of Theoretical Biology, 242 (3):607-616, 2006.

[64] A. Zemel and S. A. Safran. Active self-polarization of contractile cells in asymmetrically shaped domains. Physical Review E, 76(2):021905-1-11, 2007. 
[65] A. Zemel, F. Rehfeldt, A. E. X. Brown, D. E. Discher, and S. A. Safran. Cell shape, spreading symmetry and the polarization of stress-fibers in cells. Journal of Physics: Condensed Matter, 22(19):194110-1-20, 2010. 